\title{
Optimizing quality and efficacy of colorectal cancer diagnosis and endoscopic therapy
}

Citation for published version (APA):

van de Wetering, A. J. P. (2020). Optimizing quality and efficacy of colorectal cancer diagnosis and endoscopic therapy. [Doctoral Thesis, Maastricht University]. ProefschriftMaken. https://doi.org/10.26481/dis.20201124aw

Document status and date:

Published: 01/01/2020

DOI:

10.26481/dis.20201124aw

Document Version:

Publisher's PDF, also known as Version of record

\section{Please check the document version of this publication:}

- A submitted manuscript is the version of the article upon submission and before peer-review. There can be important differences between the submitted version and the official published version of record. People interested in the research are advised to contact the author for the final version of the publication, or visit the DOI to the publisher's website.

- The final author version and the galley proof are versions of the publication after peer review.

- The final published version features the final layout of the paper including the volume, issue and page numbers.

Link to publication

\footnotetext{
General rights rights.

- You may freely distribute the URL identifying the publication in the public portal. please follow below link for the End User Agreement:

www.umlib.nl/taverne-license

Take down policy

If you believe that this document breaches copyright please contact us at:

repository@maastrichtuniversity.nl

providing details and we will investigate your claim.
}

Copyright and moral rights for the publications made accessible in the public portal are retained by the authors and/or other copyright owners and it is a condition of accessing publications that users recognise and abide by the legal requirements associated with these

- Users may download and print one copy of any publication from the public portal for the purpose of private study or research.

- You may not further distribute the material or use it for any profit-making activity or commercial gain

If the publication is distributed under the terms of Article $25 \mathrm{fa}$ of the Dutch Copyright Act, indicated by the "Taverne" license above, 


\section{Optimizing quality}

and efficacy of

\section{colorectal cancer}

diagnosis and

\section{endoscopic therapy}

Wiesje van de Wetering 



\section{Optimizing quality and efficacy of colorectal cancer diagnosis and endoscopic therapy}

A.J.P. van de Wetering 
Copyright (C) A.J.P. van de Wetering, Maastricht 2020

All rights reserved. No parts of this thesis may be reproduced or transmitted in any form or by any means, without prior permission in writing by the author, or when appropriate, by the publishers of the publications.

ISBN:

978-94-6423-041-3

Cover design: Mariëlle Schram

Printed by: Proefschriftmaken.nl

The work presented in this thesis was performed within the framework of GROW School for Oncology and Developmental Biology (Maastricht University)

Part of the research in this thesis was financially supported by Aple d'Huzes, Dutch Cancer Society. Printing of this thesis was financially supported by Maastricht University 


\section{Optimizing quality and efficacy of colorectal cancer diagnosis and endoscopic therapy}

\section{PROEFSCHRIFT}

ter verkrijging van de graad van doctor aan de Universiteit Maastricht, op gezag van de Rector Magnificus, Prof.dr. Rianne M. Letschert volgens het besluit van het College van Decanen, in het openbaar te verdedigen op dinsdag 24 november 2020 om 10:00 uur

door

Alouisa Johanna Petronella van de Wetering Geboren op 24 mei 1991 te Oss 


\section{Promotores}

Dhr. prof. dr. A.A.M. Masclee

Mw. prof. dr. M. van Engeland

\section{Co-promotor}

Mw. dr. S. Sanduleanu

\section{Beoordelingscommissie}

Mw. prof. dr. N.D. Bouvy, voorzitter

Mw. prof. dr. I.D. Nagtegaal, Radboud Universitair Medisch Centrum

Dhr. prof. dr. J.C.H. Hardwick, Leids Universitair Medisch Centrum

Mw. prof. dr. i.r M.P. Weijenberg

Mw. dr. S. Breukink 


\section{Table of contents}

$\begin{array}{lll}\text { Chapter } 1 \text { General introduction } & 8\end{array}$

Chapter 2 Lessons from a systematic literature search on diagnostic $\mathbf{2 6}$ DNA methylation biomarkers for colorectal cancer: How to increase research value and decrease research waste Submitted

Chapter 3 Update on the role of chromoendoscopy in colonoscopic 92 surveillance of patients with Lynch syndrome European Journal of Gastroenterology and Hepatology 2018

Chapter 4 Optical diagnosis of diminutive polyps in the Dutch Bowel 116 Cancer Screening Program; are we ready to start? Endoscopy International Open 2020

Chapter 5 Optical diagnosis of T1CRCs and treatment consequences 142 in the Dutch CRC screening programme Gut 2020

Chapter 6 Multitarget stool DNA testing and Faecal Immunochemical 150 Testing (FIT) as alternative for colonoscopy-based surveillance: an interim analysis To be submitted after completion of the MOCCAS study

Chapter 7 Impact of endoscopist training on postcolonoscopy 178 colorectal cancer rate Gastrointestinal Endoscopy 2017

Chapter 8 General discussion

Addendum Summary

Samenvatting

Valorization

List of publications

Curriculum Vitae

Dankwoord 



\section{CHAPTER 1}

General introduction \&

Aims and outline 


\section{General introduction}

\section{Colorectal cancer}

Colorectal cancer (CRC) belongs to the top three most commonly occurring cancers worldwide in both women and men and contributes substantially to cancerrelated mortality and morbidity, with 14.1 million new cases and 8.2 million deaths worldwide in 2012.1,2 CRC arises from precursor lesions that usually grow slowly. ${ }^{3,4}$ Incidence, morbidity and mortality of CRC can be reduced by early detection and (endoscopic) removal of these precursor lesions and CRCs. ${ }^{5}$ Therefore, CRC is suitable for population-based screening. In 2015, 24 European countries had established or were preparing to organize a nationwide CRC screening program. ${ }^{6}$ Current CRC screening programs are based on invasive techniques as flexible sigmoidoscopy or colonoscopy, or based on stool tests: guaiac fecal occult blood test (G-FOBT) and the fecal immunochemical test (FIT), where FIT has been recommended as first option. ${ }^{6,7}$

\section{Dutch colorectal cancer screening program}

In 2014 the CRC screening program was introduced in the Netherlands. Participants between 55 and 75 years old are being invited for screening using FIT. ${ }^{8}$ In case the FIT result equals or exceeds the cut-off level (initially at $15 \mu \mathrm{g}$ haemoglobin per gram feces; six months after implementation raised from $15 \mu \mathrm{g}$ to $47 \mu \mathrm{g}$ haemoglobin per gram feces because of limited endoscopy capacity) participants are referred for colonoscopy. All colonoscopies are performed by accredited endoscopists who perform at least 300 colonoscopies yearly. ${ }^{9}$ Lesions found during colonoscopy are removed and histologically evaluated. In case of an advanced adenoma (adenoma $\geq 10 \mathrm{~mm}$ and/or with high grade dysplasia and/or $\geq 25 \%$ villous component) or CRC, the participant will be referred for further treatment and surveillance. ${ }^{10}$ 


\section{Colonoscopy and surveillance}

Colonoscopy is an important tool for the detection and removal of precursor lesions. Colonoscopy allows direct visualization of the lesion while providing the ability to take biopsies or even resect the lesion. Although the risk of colonoscopy complications is low, bleeding and perforation can still occur. In screening and surveillance settings, perforation rates of $0.03 \%$ and bleeding rates of $0.24 \%$ are reported, being significantly lower compared to symptomatic colonoscopies. These numbers increase after polypectomy to $0.08 \%$ and $0.98 \%$ respectively. ${ }^{11}$

In addition, it needs to be kept in mind that colonoscopy is an invasive procedure, potentially with significant burden for the patient. Based on outcomes of questionnaires among screening program participants in the Netherlands, bowel preparation and post-colonoscopy abdominal complaints are perceived as most burdensome. ${ }^{12}$

With implementation of the Dutch CRC screening program, a pre-selected group of asymptomatic participants (55-75 years, unfavorable FIT) undergo a colonoscopy procedure, which lead to an estimated 72.000 extra colonoscopies yearly in 20142020 in the Netherlands. ${ }^{13}$ In 2019, the stepwise implementation of the CRC screening program in the Netherlands has been completed, i.e. every potentially eligible person has been invited to participate. ${ }^{13}$ The Netherlands has the highest participation rate $(68.2 \%)^{6}$ and the demand for colonoscopy capacity will extend further since participants with relevant lesions will enter the surveillance program. The cost-benefit of the current colonoscopy-based surveillance programs is however not yet clear $^{14}$ because of the major impact on colonoscopy capacity. Eventually more than $25 \%$ of colonoscopies will be performed for reasons of post-polypectomy surveillance. ${ }^{15,16}$ This will lead to higher healthcare costs and is therefore a financial and capacity burden to society. On the other hand, significant costs are saved from chemotherapy and surgery. Most importantly, due to CRC screening significantly less people will develop (advanced) CRC and die from CRC and its complications. On the long-term it is anticipated that nationwide CRC screening will become cost effective. ${ }^{17}$ 
It is therefore important to optimize the pre-selection of participants who are invited for a screening or surveillance colonoscopy in order to lower the burden for participants as well as for clinicians.

\section{Biomarkers}

Several methods that will help the process of a better pre-selection of patients who actually need a (screening) colonoscopy and thereby decreasing the number and burden of the colonoscopy capacity are currently being investigated. As mentioned above, FIT is widely implemented for this purpose. FIT is reported to be $68-87 \%$ sensitive and $96-98 \%$ specific for $\mathrm{CRC}^{18,19}$ However, for precursor lesions, i.e. advanced adenomas, the sensitivity is substantially lower (23-40\%). . $^{80}$

The process of carcinogenesis in CRC is related to different mechanisms, among which gene mutations (i.e. mutations in BRAF, KRAS and p53 and microsatelite instability) and epigenetic alterations (i.e. DNA methylation of $\mathrm{CpG}$ island promotor regions), creating opportunities for early diagnosis using molecular markers.

Molecular markers, e.g. DNA methylation markers, appear to hold great potential for detection of precursor lesions and early CRC diagnosis as promoter hyper methylation is a common event during colorectal cancer tumorgenesis and occurs early in cancer development. While a variety of DNA methylation markers is being evaluated in different tissues or bodily fluids (colonic tissue, blood, stool, urine), only three commercially available assays designed to measure DNA methylation based CRC biomarkers are currently on the market (Cologuard $®$ (NDRG4, BMP3), Epi proColon/ColoVantage ${ }^{\circledR}$ (Licensed to Quest Diagnostics and ARUP Laboratories) and Epi proColon® (both SEPTIN9)). ${ }^{21}$ This translational efficiency illustrates the gap between research and clinical practice.

\section{Quality of colonoscopy}

Another option to reduce the financial and patient burden is to optimize current colonoscopy quality. Performance measures for colonoscopy have been set both nationally and internationally, are incorporated into guidelines and are used as quality indicators such as: 1 ) adequate bowel preparation in $\geq 90 \%$ of the 
colonoscopies (defined as Boston Bowel Preparation Score (BBPS) $\geq 6$, with a minimum of 2 for each segment), 2) cecal intubation rate ( $\geq 90 \%$ ), 3) adenoma detection rate (detection of $\geq 1$ adenoma in $\geq 25 \%$ of the patients), 4) appropriate polypectomy technique ( $\geq 80 \%$ choice of adequate resection technique according to the ESGE guideline (since inappropriate polypectomy technique increases the risk of incomplete polyp removal) and 5) minimum withdrawal time of $\geq 6$ minutes. In addition, complication rate, patient experiences and outcome measures and appropriate post-polypectomy surveillance recommendations are also of importance. $^{22}$ These parameters are continuously monitored and need to be reported at endoscopist level, centre level and at national level. ${ }^{23} \mathrm{New}$ endoscopic techniques with image enhancement and chromoendoscopy further facilitate the diagnostic and therapeutic efficacy of colonoscopy.

\section{Image-enhancement}

In most colonoscopy practices in the Western world, high-definition (HD) whitelight endoscopy (WLE) has become gold-standard and has been shown to enhance the detection of colorectal polyps ${ }^{24}$ (Figure 1-left). In addition, advanced imaging techniques, e.g. (virtual) chromoendoscopy (CE) provides the opportunity to evaluate polyps in more detail and is very helpful for trained endoscopists to make a reliable optical diagnosis for colonic lesions $\mathrm{s}^{25}$, and differentiating between neoplastic and non-neoplastic polyps. ${ }^{26}$

Modalities as narrow-band imaging (NBI), autofluorescence endoscopy (AFE), Fuji Intelligent ChromoEndoscopy (FICE) and I-scan have incorporated features to enhance visualization of the surface structures and vascular patterns of the mucosal layer ${ }^{27}$ (Figure 1-middle), whereas these structures and patterns are altered in (pre) cancerous lesions. ${ }^{28}$ Dye-based chromoendoscopy, where a blue color is sprayed over the mucosal surface, forms pools in areas of depth, thereby highlighting subtle depressions and borders of the abnormal tissue that facilitates adenoma detection ${ }^{29}$ (Figure 1-right). Image-enhancement is also relevant in optimizing the diagnosis of T1 CRC and T1 CRC with deep submucosal invasion. ${ }^{30}$ 


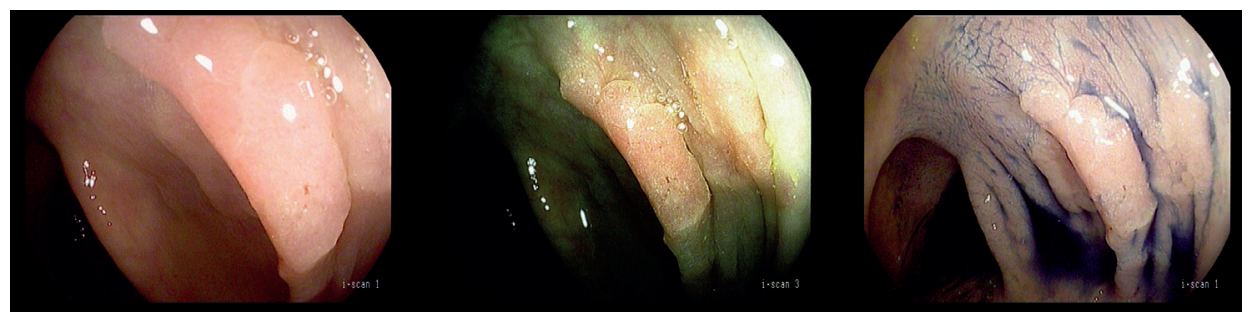

Figure 1. Overview of the different image enhancement techniques, showing a non-granular laterally spreading tumor in the proximal colon of a 44-year old male with Lynch Syndrome. From left to right: White-light endoscopy (high-definition), Virtual chromoendoscopy (I-scan), Dye-based chromoendoscopy with indigo carmine.

\section{Optical diagnosis}

In current clinical practice, all lesions visually detected during colonoscopy are resected and histologically analysed. Based on the histological results (i.e. nature of the lesion, advanced histological features and potential invasive growth) surveillance recommendations are made. ${ }^{31,32}$ However, histological evaluation is costly, especially since the great majority of polyps found during colonoscopy are diminutive polyps $(\leq 5 \mathrm{~mm})$, which very rarely harbor advanced histological features or CRC. ${ }^{33}$

Optical diagnosis, that is the real-time (correct) endoscopic assessment of histology of the polyp by the endoscopist ${ }^{34}$, is suggested to be a more cost-effective strategy. ${ }^{35,36}$ However, before optical diagnosis of diminutive polyps can be safely implemented in clinical practice, the 'Preservation and Incorporation of Valuable endoscopic Innovations' (PIVI) thresholds should be met. These PIVI criteria have been designed and proposed as a basis to be met in order to safely implement the 'resect and discard' strategy (i.e. resect diminutive colorectal adenomatous polyps, but these lesions are not sent for histopathological evaluation) and the 'diagnose and leave' strategy (i.e. diminutive hyperplastic polyps in the rectosigmoid are identified and left in situ). 
The PIVI thresholds suggest $\geq 90 \%$ negative predictive value for adenomatous histology and $\geq 90 \%$ agreement between optical diagnosis and histological diagnosis in determining the post-polypectomy surveillance intervals. ${ }^{37}$

In dedicated expert settings, these PIVI thresholds come into sight. It remains to be determined whether these strategies based on endoscopist optical diagnosis can eventually be implemented in daily endoscopy practice.

\section{T1 (early) cancers}

An evaluation of all CRC patients in the Netherlands between 60 and 75 years has shown that CRCs detected via the Dutch CRC screening program were more often early stage compared to symptom-detected CRCs (48.2\% stage I CRC (T1-2, N0M0) in all screen-detected CRCs vs. $16.7 \%$ stage I CRC in all symptom-detected CRCs). ${ }^{38}$ Given the shift towards CRC detection at earlier stages, it is important to optimize diagnosis and treatment of these early stage cancer patients.

The first crucial step in optimizing the management for T1 CRC (defined as tumors with invasion into but not beyond the submucosa ${ }^{39}$ ) is recognition. Accurate optical diagnosis is crucial to define which resection technique is optimal. ${ }^{40}$

In the past decades surgical excision has been considered the optimal treatment for CRC. Nowadays for the increasing subset of T1 CRCs, endoscopic resection techniques are becoming increasingly important.

Endoscopic mucosal resection $(E M R)^{41}$ and endoscopic submucosal dissection $(E S D)^{42}$ allow complete resection of colorectal lesions while preserving bowel wall integrity. Transanal endoscopic microsurgery (TEM) is a minimal invasive technique applicable to rectal lesions. These minimal invasive local excision techniques harbor substantially lower complication and mortality rates compared to surgery ${ }^{43,44}$ but are technically demanding, time consuming and require substantial endoscopist expertise. 


\section{Post colonoscopy colorectal cancers}

In both diagnosis and treatment of (pre-cancerous) lesions, colonoscopy is important. Although colonoscopy is considered a safe and effective strategy to prevent CRC, it is not perfect. With respect to optimizing colonoscopy quality, several factors should be considered such as adequate bowel preparation, cecal intubation rate, adenoma detection rate and minimum withdrawal time.

Postcolonoscopy colorectal cancer (PCCRC) rate can also be seen as an indicator of quality. If CRC is diagnosed after a previously performed negative colonoscopy, it is called a PCCRC. There is some variation in the definition of PCCRCs. The maximum time interval between a negative colonoscopy and the CRC diagnosis varies from 36 to 120 months, reported in the literature. ${ }^{45,46}$ All CRCs diagnosed between 6 to 60 months after a previous colonoscopy that was negative for CRC are currently defined as PCCRC. ${ }^{47}$

Several causes for PCCRCs can be identified. Since patients with relevant lesions are invited for surveillance according to guidelines, non-adherence to these surveillance intervals can be a reason for development of PCCRC. When the quality of colonoscopy is not sufficient, i.e. inadequate bowel preparation or incomplete colonoscopy, this can also contribute to PCCRC development since (high-risk) lesions can be missed. If a polyp is not completely resected, the residue which may develop into carcinoma, also contributes to PCCRC occurrence. In addition, some PCCRCs develop from missed lesions for which the reason is not completely clear. Newly developed lesions can also attribute to PCCRCs, where it is possible that precursor lesions harbor specific biological features, resulting in rapid progression of a new precursor lesions leading to PCCRC. ${ }^{48}$ 


\section{Aims and outline of this thesis}

The process of optimizing quality and efficacy of colorectal cancer diagnosis and endoscopic therapy is extensive and includes many aspects. In this thesis we have focused on pre-selection, colonoscopy, minimal invasive treatment and surveillance for colorectal cancer (Figure 2).

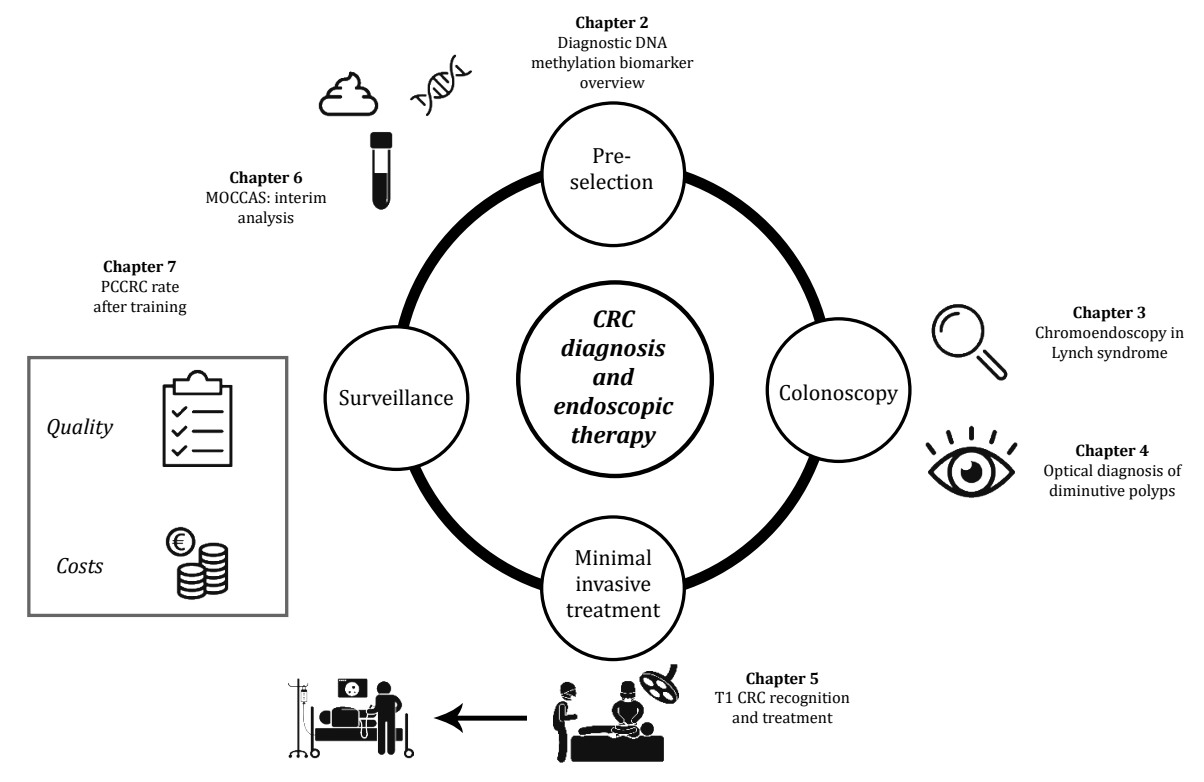

Figure 2. Aims and outline of this thesis 


\section{Pre-selection}

As described above, it is important to identify the best, and least invasive way to preselect subjects referred for colonoscopy. In the current Dutch CRC screening program, FIT is being used for pre-selection. FIT is sensitive and specific for CRC, however it is not so sensitive for precursor lesions, i.e. advanced adenomas. Therefore, it is worthwhile to investigate alternative non-invasive tests.

In the United States, methylation marker tests are used for pre-selection. DNA methylation markers are very promising and are currently under extensive investigation with the aim of accurate and early detection of (pre)cancerous lesions. Chapter 2 provides an extensive overview of the current status in the research field of DNA methylation markers covering the period since the first biomarker was published (1985) up to 2019. Although a significant number of markers has been studied, only a few are being used in clinical practice. This overview summarizes on all the relevant biomarkers in tissue and in bodily fluids (stool, blood, urine) that have been published. In addition, study recommendations and markers considered to become relevant in the near future are being discussed.

\section{Colonoscopy}

Colonoscopy is currently the gold standard for detection of colorectal lesions. To further improve the (optical) diagnosis of colorectal lesions, image-enhancement techniques are supposed to be of additional value.

In chapter 3 the additional value of (virtual) chromoendoscopy is reviewed in patients with Lynch Syndrome (LS), a high-risk group with a genetic predisposition harboring an increased CRC risk. Since it is known that flat and depressed (non-polypoid) lesions are important contributors to (interval) CRC in LS patients, and detection of these lesions is challenging, an update on current use of image-enhancement in this high-risk group is provided. 
With the implementation of the Dutch CRC screening program, more colonoscopies are performed and more (smaller) lesions are visualized. Chapter 4 focusses on the optical diagnosis of diminutive polyps $(\leq 5 \mathrm{~mm})$, where the accuracy of optical diagnosis by the endoscopist is compared to histological evaluation for diminutive polyps. In addition, two strategies are considered. The 'resect-and-discard' strategy, where optical diagnosis is suggested to replace histological evaluation for diminutive polyps and the 'diagnose-and-leave' strategy, where non-neoplastic polyps in the rectosigmoid could be left in place. The thresholds for implementation of these strategies (i.e. PIVI) are investigated.

\section{Minimal invasive treatment}

Not only small and diminutive polyps are seen more often with implementation of the Dutch CRC screening program, also more early cancers (T1 CRCs) are diagnosed. Chapter 5 provides an update on recognition and treatment of T1 CRCs in the Dutch CRC screening program. To provide the optimal treatment for the individual patient, adequate recognition of T1 CRCs is crucial.

We evaluated the accuracy of optical diagnosis for T1 CRC in a large, well phenotyped cohort from South Limburg that is representative for the nationwide screening program in the Netherlands. Additionally, treatment decisions and follow-up are assessed in current clinical practice. This chapter gives an overview of real-life practice in the first years of the Dutch CRC screening program. The recognition of T1 CRCs is assessed and treament consequences are evaluated. 


\section{Surveillance}

When patients have a familial CRC risk or history of CRC or polypectomy, they enter the surveillance program. Surveillance colonoscopies are a substantial part of the total colonoscopy capacity and a major burden for the patient and healthcare system, while it is questionable whether these colonoscopies are cost-effective. In chapter 6 the interim analysis of the MOCCAS (MOlecular stool testing for Colorectal CAncer Surveillance) study is presented, where the multitarget stool DNA (mt-sDNA) test comprising DNA methylation and mutation markers (Cologuard®, Exact Sciences, Madison, WI, USA) is compared to the fecal immunochemical test (FIT). Aim is to assess whether this mt-sDNA test could be used to preselect patients with advanced neoplasia for surveillance colonoscopy.

Quality control and monitoring is important during every step of the process in optimizing diagnosis, treatment and surveillance for CRC. An important indicator of effectiveness and quality of CRC screening and surveillance are PCCRCs. ${ }^{47}$ The factors attributable to PCCRCs are widely researched, as well as strategies to decrease the PCCRC rate. In chapter 7 the impact of endoscopist training on lowering the PCCRC rate in Maastricht UMC+ is shown over time. 


\section{References}

1. Siegel RL, et al. Cancer statistics, 2019. CA Cancer J Clin 2019;69(1):734.

2. Torre LA, et al. Global cancer statistics, 2012. CA Cancer J Clin 2015;65(2):87-108.

3. Vogelstein B, et al. Genetic alterations during colorectaltumor development. N Engl J Med 1988;319(9):525-32.

4. Muto T, et al. The evolution of cancer of the colon and rectum. Cancer 1975;36(6):2251-70.

5. Winawer SJ, et al. Prevention of colorectal cancer by colonoscopic polypectomy. The National Polyp Study Workgroup. N Engl J Med 1993;329(27):1977-81.

6. Navarro M, et al. Colorectal cancer population screening programs worldwide in 2016: An update. World J Gastroenterol 2017;23(20):3632-42.

7. Tinmouth J, et al. Faecal immunochemical tests versus guaiac faecal occult blood tests: what clinicians and colorectal cancer screening programme organisers need to know. Gut 2015;64(8):132737.

8. Toes-Zoutendijk E, et al. Real-Time Monitoring of Results During First Year of Dutch Colorectal Cancer Screening Program and Optimization by Altering Fecal Immunochemical Test CutOff Levels. Gastroenterology 2017;152(4):767-75 e2.
9. Rijksinstituut voor Volksgezondheid en Mileu (RIVM). Protocol voor de toelating en auditing van coloscopiecentra en endoscopisten - Bevolkingsonderzoek Darmkanker. 2012.

10. Dekker E, et al. Nederlandse Richtlijn Coloscopie Surveillance. NVMDL ism Nederlandse Vereniging van Pathologie. 2013. Available from: http://www.mdl.nl/upload. 2013.

11. Reumkens A, et al. Post-Colonoscopy Complications: A Systematic Review, Time Trends, and Meta-Analysis of Population-Based Studies. Am J Gastroenterol 2016;111(8):1092101.

12. Denters MJ, et al. Patient burden of colonoscopy after positive fecal immunochemical testing for colorectal cancer screening. Endoscopy 2013;45(5):342-9.

13. Rijksinstituut voor Volksgezondheid en Milieu (RIVM). Bevolkingsonderzoek Darmkanker. Available via: http://www.rivm.nl/.

14. Greuter MJE, et al. Screening for Colorectal Cancer With Fecal Immunochemical Testing With and Without Postpolypectomy Surveillance Colonoscopy: A CostEffectiveness Analysis. Ann Intern Med 2017;167(8):544-54. 
15. Gavin DR, et al. The national colonoscopy audit: a nationwide assessment of the quality and safety of colonoscopy in the UK. Gut 2013;62(2):242-9.

16. Lieberman D. Colon-polyp surveillance--do patients benefit? N Engl J Med 2014;371(9):860-1.

17. Ladabaum $U$, et al. Real-time optical biopsy of colon polyps with narrow band imaging in community practice does not yet meet key thresholds for clinical decisions. Gastroenterology 2013;144(1):81-91.

18. Oort FA, et al. Colonoscopycontrolled intra-individual comparisons to screen relevant neoplasia: faecal immunochemical test vs. guaiac-based faecal occult blood test. Aliment Pharmacol Ther 2010;31(3):432-9.

19. Gies A, et al. Direct Comparison of Diagnostic Performance of 9 Quantitative Fecal Immunochemical Tests for Colorectal Cancer Screening. Gastroenterology 2018;154(1):93-104

20. Imperiale TF, et al. Performance Characteristics of Fecal Immunochemical Tests for Colorectal Cancer and Advanced Adenomatous Polyps: A Systematic Review and Meta-analysis. Ann Intern Med 2019.

21. Koch A, et al. Analysis of DNA methylation in cancer: location revisited. Nat Rev Clin Oncol 2018;15(7):459-66.
22. Kaminski MF, et al. Performance measures for lower gastrointestinal endoscopy: a European Society of Gastrointestinal Endoscopy (ESGE) Quality Improvement Initiative. Endoscopy 2017;49(4):378-97.

23. Bronzwaer MES, et al. Quality assurance of colonoscopy within the Dutch national colorectal cancer screening program. Gastrointest Endosc 2019;89(1):1-13.

24. Rex DK, Helbig CC. High yields of small and flat adenomas with highdefinition colonoscopes using either white light or narrow band imaging. Gastroenterology 2007;133(1):42-7.

25. Wanders LK, et al. Diagnostic performance of narrowed spectrum endoscopy, autofluorescence imaging, and confocal laser endomicroscopy for optical diagnosis of colonic polyps: a meta-analysis. Lancet Oncol 2013;14(13):1337-47.

26. McGill SK, et al. Narrow band imaging to differentiate neoplastic and non-neoplastic colorectal polyps in real time: a meta-analysis of diagnostic operating characteristics. Gut 2013;62(12):1704-13.

27. Kaminski MF, et al. Advanced imaging for detection and differentiation of colorectal neoplasia: European Society of Gastrointestinal Endoscopy (ESGE) Guideline. Endoscopy 2014;46(5):435-49 
28. Hazewinkel Y \& Dekker E. Colonoscopy: basic principles and novel techniques. Nat Rev Gastroenterol Hepatol 2011;8(10):554-64.

29. McGill S, et al. Image-enhanced endoscopy in practice. Can J Gastroenterol 2009;23(11):741-6.

30. Backes Y, et al. Narrow Band Imaging, Magnifying Chromoendoscopy, and Gross Morphological Features for the Optical Diagnosis of T1 Colorectal Cancer and Deep Submucosal Invasion: A Systematic Review and Meta-Analysis. Am J Gastroenterol 2017;112(1):54-64.

31. Hassan C, et al. Post-polypectomy colonoscopy surveillance: European Society of Gastrointestinal Endoscopy (ESGE) Guideline. Endoscopy 2013;45(10):842-51.

32. Lieberman DA, et al. Guidelines for colonoscopy surveillance after screening and polypectomy: a consensus update by the US Multi-Society Task Force on Colorectal Cancer. Gastroenterology 2012;143(3):844-57.

33. Ponugoti PL, et al. Risk of cancer in small and diminutive colorectal polyps. Dig Liver Dis 2017;49(1):347.
34. ASGE Committee, et al. ASGE Technology Committee systematic review and meta-analysis assessing the ASGE PIVI thresholds for adopting real-time endoscopic assessment of the histology of diminutive colorectal polyps. Gastrointest Endosc 2015;81(3):502 e1- e16.

35. Hassan C, et al. A resect and discard strategy would improve costeffectiveness of colorectal cancer screening. Clin Gastroenterol Hepatol 2010;8(10):865-9, 9 e1-3.

36. Vleugels JLA, et al. Implementation of an optical diagnosis strategy saves costs and does not impair clinical outcomes of a fecal immunochemical test-based colorectal cancer screening program. Endosc Int Open 2017;5(12):E1197-E207.

37. Rex DK, et al. The American Society for Gastrointestinal Endoscopy PIVI (Preservation and Incorporation of Valuable Endoscopic Innovations) on real-time endoscopic assessment of the histology of diminutive colorectal polyps. Gastrointest Endosc 2011;73(3):419-22.

38. Toes-Zoutendijk E, et al. Stage distribution of screen-detected colorectal cancers in the Netherlands. Gut 2018;67(9):17456.

39. Sobin L. TNM Classification of Malignant Tumours. West Sussex, United Kingdom, Wiley-Blackwell 2009. 
40. Backes Y, et al. Multicentre prospective evaluation of real-time optical diagnosis of $\mathrm{T} 1$ colorectal cancer in large non-pedunculated colorectal polyps using narrow band imaging (the OPTICAL study). Gut 2019;68(2):271-9.

41. Soetikno RM, et al. Endoscopic mucosal resection. Gastrointest Endosc 2003;57(4):567-79.

42. Tamegai Y, et al. Endoscopic submucosal dissection: a safe technique for colorectal tumors. Endoscopy 2007;39(5):418-22.

43. Fujiya M, et al. Efficacy and adverse events of EMR and endoscopic submucosal dissection for the treatment of colon neoplasms: a meta-analysis of studies comparing EMR and endoscopic submucosal dissection. Gastrointest Endosc 2015;81(3):583-95.

44. Ahlenstiel G, et al. Actual endoscopic versus predicted surgical mortality for treatment of advanced mucosal neoplasia of the colon. Gastrointest Endosc 2014;80(4):668-76.

45. Stoffel EM, et al. Clinical and Molecular Characteristics of Post-Colonoscopy Colorectal Cancer: A Population-based Study. Gastroenterology 2016;151(5):8708 e3.

46. Singh S, et al. Prevalence, risk factors, and outcomes of interval colorectal cancers: a systematic review and meta-analysis. Am J Gastroenterol 2014;109(9):1375-89.
47. Sanduleanu S, et al. Definition and taxonomy of interval colorectal cancers: a proposal for standardising nomenclature. Gut 2015;64(8):1257-67.

48. Pabby A, et al. Analysis of colorectal cancer occurrence during surveillance colonoscopy in the dietary Polyp Prevention Trial. Gastrointest Endosc 2005;61(3):385-91. 
GENERAL INTRODUCTION 



\section{CHAPTER 2}

\section{Lessons from a systematic literature search on diagnostic DNA methylation biomarkers for colorectal cancer: How to increase research value and decrease research waste}

Alouisa J.P. van de Wetering*, Zheng Feng*, Cary J.G. Oberije*, Alexander Koch, Nathalie Vaes, Ad A. M. Masclee, Beatriz Carvalho, Gerrit A. Meijer, Maurice P. Zeegers, James G. Herman, Veerle Melotte, Manon van Engeland, Kim M. Smits

*Shared first authorship 


\section{Abstract}

\section{Background}

To improve colorectal cancer (CRC) survival, screening with colonoscopy and/or faecal immunochemical tests (FIT) is widely implemented. Although candidate DNA methylation biomarkers have been published to improve or complement the FIT, clinical translation is limited.

Here, we describe technical and methodological problems encountered after a systematic literature search and provide recommendations to increase (clinical) value and decrease research waste in biomarker research. In addition, we present the current evidence for diagnostic CRC DNA methylation biomarkers.

\section{Methods}

A systematic literature search was performed, identifying 273 diagnostic DNA methylation marker studies published before January 2019 in PubMed, Embase, Cochrane Library or Google Scholar; for 109 bodily fluid biomarker studies, extended data extraction (study design, patient characteristics, disease stage, tumour location, technical assays, diagnostic measures) was performed on published reports. STARD criteria and Level of Evidence (LoE) were registered to assess reporting quality and strength for clinical translation.

\section{Findings}

Our systematic literature search revealed multiple issues, that hamper the development of DNA methylation biomarkers for CRC diagnosis, including methodological and technical heterogeneity and lack of validation or clinical translation. For example, clinical translation and independent validation was limited, with $88 / 389(23 \%)$ markers studied in bodily fluids, 3/389 (0.8\%) translated into a clinical test, and independent validation for 78/389 (20\%) tissue markers and $37 / 88$ (42\%) bodily fluids markers.

\section{Interpretation}

This systematic literature search revealed that major requirements to develop clinically relevant diagnostic CRC DNA methylation markers are often lacking. To avoid the resulting research waste clinical needs, intended biomarker use and independent validation should be better considered prior to study design. In addition, improved reporting quality would facilitate meta-analysis, thereby increasing LoE and enabling clinical translation.

\section{Funding}

Funding was provided by "Maag Lever Darm Stichting"(MLDS-project FP13-15) and a SU2C-DCS International Translational Cancer Research Dream Team Grant (StandUp-To-Cancer(SU2C)-AACR-DT1415,MEDOCC). 


\section{Introduction}

Colorectal cancer (CRC) is the third most common cancer worldwide and a major contributor to cancer-related mortality. ${ }^{1}$ In 2018, incidence was 1.8 million and over 881.000 people died from CRC. ${ }^{2}$ By 2030, this is expected to increase to over 2.2 million new cases and 1.1 million deaths ${ }^{3}$ illustrating the heavy patient and societal burden. ${ }^{4}$ Early detection and treatment of CRC and its high-risk precursor lesions leads to reduced mortality, morbidity, and healthcare costs. ${ }^{5}$ Therefore, CRC screening programs have been widely implemented. ${ }^{6}$

Currently, colonoscopy is the gold standard method to diagnose CRC, allowing to take biopsies and resect qualifying (pre-)cancerous lesions directly. However, it is invasive with a high patient burden, mainly caused by the required bowel preparation and post-colonoscopy abdominal symptoms. ${ }^{7}$ Occasionally, severe complications occur, such as bleeding $(0.26 \%)$ and perforation $(0.05 \%){ }^{8}$

Colonoscopy numbers increase rapidly with screening, inherently yielding an increase in surveillance demand after removal of (pre-)cancerous lesions. ${ }^{9}$ This already consumes about $25 \%$ of total colonoscopy capacity. ${ }^{10,11}$ To lower the burden for patients and clinicians and avoid complications, pre-selection of patients for colonoscopy should be optimized.

The most common pre-selection test is the faecal immunochemical test (FIT), measuring stool haemoglobin levels. ${ }^{6}$ However, as FIT sensitivities, especially for advanced adenomas, are suboptimal ${ }^{12,13}$ and false-positive rates can be high ${ }^{14}$, there is an ongoing search for biomarkers to complement FIT. Many candidates to improve this pre-selection have been reported, amongst which DNA methylation markers ${ }^{15-18}$ in tissue, blood, urine or stool. ${ }^{19-21}$

However, only three CRC detection tests measuring DNA methylation markers are currently commercially available: Cologuard $\AA$ (including NDRG4 and BMP3 methylation), EpiproColon/ColoVantage ${ }^{\circledR}$ (Licensed to Quest Diagnostics and ARUP Laboratories) and EpiproColon $\AA$ (both SEPTIN9 methylation). ${ }^{22}$ This illustrates the current gap between discovery and clinical implementation. 
To improve the clinical translation of diagnostic CRC DNA methylation markers, it is important to understand the clinical needs, the status of previous research, and to know which markers have a diagnostic accuracy that might improve currently used tests. Our systematic literature search was performed to provide this evidence. In addition, we aimed to assess the specific issues that still hinder the clinical development of potential DNA methylation biomarkers and define recommendations to overcome these issues.

\section{Eligibility criteria, search strategy and selection}

A systematic review was performed to identify studies on diagnostic CRC DNA methylation markers, applying the Preferred Reporting Items for Systematic Review and Meta-Analysis of Diagnostic Test Accuracy Studies (PRISMA-DTA) guidelines for the literature search. ${ }^{23}$ Original, English language articles were considered; reviews, editorials, and conference abstracts were excluded. Articles were included if patients or cancer tissues were compared to healthy controls or non-cancerous tissues. Inclusion was not restricted to specific study designs, patient characteristics, tumor subtype, disease stage, or included patient number. Studies on hereditary cancer syndromes were excluded.

PubMed, Embase, Cochrane library and Google Scholar was searched up to January 1st 2019, with no additional limits defined (Supplementary Table 1). Reference lists of included articles were searched for additional relevant publications. Finally, 273 articles were included.

\section{Data extraction}

Four researchers (ZF, KS, AvdW, CO) performed data extraction, using a standardized data registration form. Each article was assessed independently by two researchers. If discrepancies arose, a third researcher was asked to mediate to reach consensus and take the final decision. Restricted data extraction (author, year of publication, methylation markers, specimen type) based on abstract information was performed for all 273 studies. 
Full data extraction (study design, patient characteristics, disease severity, tumor location, DNA isolation and DNA methylation method, primer/probe sequences, and diagnostic measures such as sensitivity, specificity) was performed for 109 articles on bodily fluids markers, as only non-invasive diagnostic markers are clinically relevant (Supplementary Figure 1, Supplementary Table 3).

\section{Reporting quality and Level of Evidence}

STARD 2015 criteria were adjusted for biomarker research and used to assess reporting quality ${ }^{24}$ (Supplementary Table 2).

One point was assigned for reported items, 0.5 points for incomplete reported items and zero points if items were not reported. The maximum score was 22 points. STARD scores were also used to analyze reporting quality of specific categories (i.e. population selection, assay method, outcome assessment and other variable assessment) (Supplementary Table 2 \& Supplementary Table 5).

If a study scored $\geq 0.5$ points per item for STARD items $5-9$, reporting of population selection was considered as insufficient, a score $<0.5$ increased this risk. Measurement reporting quality was measured similarly, using STARD items 10a, 12a and 13a for assay method and STARD items 14, 21a and 24 for outcome assessment.

Other variable assessment was based on STARD item 20. With a full or partial score, measurement reporting was assessed as sufficient. If this item was not reported, measurement reporting was insufficient. To grade the Level of Evidence (LoE), we used a ranking scheme adapted for biomarkers ${ }^{24,25}$ and the Oxford Centre for Evidence Based Medicine (OCEBM) 2011 Levels of Evidence. ${ }^{26}$ 


\section{Diagnostic CRC biomarkers throughout the years}

The first report on DNA methylation markers (POMC methylation) in tissue was published in 1985. Since then, numbers have been increasing (Figure 1). In total, 273 studies on 389 DNA methylation markers (Figure $1 a$ and $1 b$ ) were identified. Of these, 301/389 markers (77.4\%) were investigated in tissue, 70/389 (18.0\%) in tissue and bodily fluids and 18/389 (4.6\%) in bodily fluids only. Numbers of published tissue markers showed a peak in 2016, and decline thereafter, possibly reflecting a shift of interest to bodily fluids, as these peak after 2016.

In addition, it could also reflect a shift of interest to other biomarker types that were not assessed in this review (Supplementary Figure 2).

The first report on DNA methylation markers in bodily fluids (TMEFF2 (TPEF) methylation), appeared in 2003 (Figure 1b). Since then, 109 studies (39.9\%) reported diagnostic DNA methylation markers in bodily fluids while 164 studies (60.1\%) focused on tissue (Figure $1 a$ and $1 b$ ). These numbers illustrate that, despite the growing interest in non-invasive markers, many studies still solely focus on tissue. In our view, this is surprising as diagnostic CRC biomarkers are developed to preselect patients for colonoscopy and avoid unnecessary invasive procedures.

Next to lack of translation from tissue to bodily fluids, independent validation (i.e. evaluating biomarkers in other studies or study populations) rarely occurs. Only 90/389 markers (23.1\%) were independently validated. In tissue, 78/371 markers (21.0\%) were studied at least twice, CDKN2A (p16) and MGMT were most frequently studied (16 times) (Figure $2 a$ ).

In bodily fluids, 37/88 markers (42.0\%) were studied more than once. SEPTIN9 was most frequently analyzed and assessed in 26 studies. SFRP2,VIM, CDKN2A (p16) and MGMT were analyzed in 15, 11, 7 and 6 studies respectively (Figure 2b). In contrast, 12 other markers were only validated twice indicating that even if independent validation was performed, this was done in a limited number of validation sets. ${ }^{27}$ 
Figure 1. Yearly identified (1985-2018) DNA methylation biomarkers for CRC detection in tissue and body fluid samples

(a) Tissue biomarkers

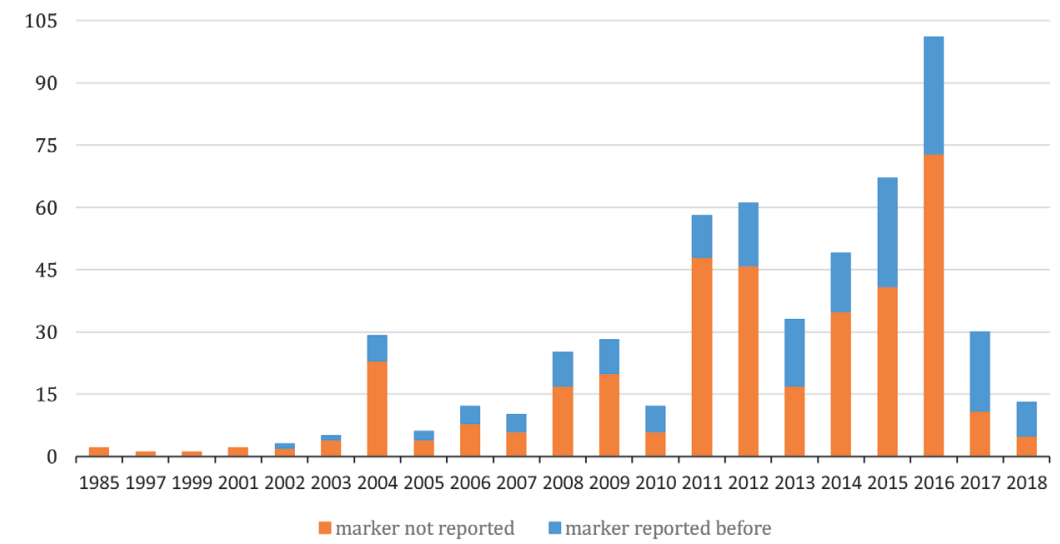

(b) Bodily fluid biomarkers

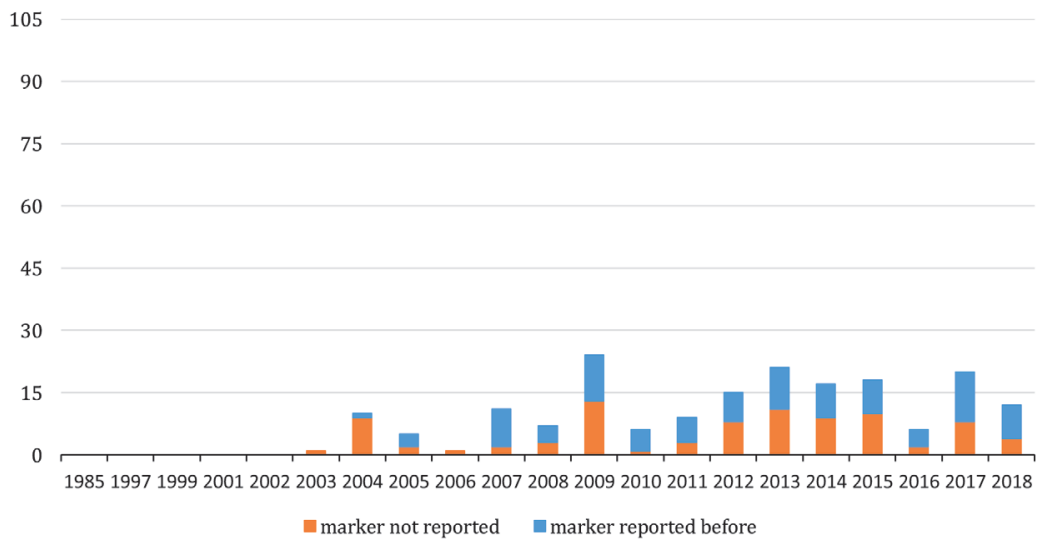

The length of bars denotes total number of biomarkers investigated across all selected studies per year. Each bar is divided into two categories, where the orange color indicates the number of biomarkers newly identified in that year and the blue color represents the number of biomarkers discovered in previous years. 
Internal validation was often done by splitting sample sets. This approach should however be avoided, especially in small populations where bootstrapping might be more suitable. ${ }^{28}$

Interestingly, independent validation was independent of initial results. For $M G M T$ methylation in stool e.g., initially, a low sensitivity and specificity of 14.1 and $79.6 \%$ was reported. Nevertheless, this marker was studied in five subsequent studies with variable study methods, showing sensitivities ranging from 5.7-90.0\%. These large ranges can probably not be fully explained by biology, technical aspects such as assay design and resolution differences between technologies also influence these results. In contrast, DNA methylation markers showing more promising initial results, e.g. SNCA (sensitivity $70 \%$, specificity $100 \%$ ) have not been independently validated yet.

Independent validation is crucial in biomarker research ${ }^{29}$ to verify initial findings that often are too optimistic due to multiple testing in a limited dataset. ${ }^{30}$ SFRP2 methylation in stool e.g., was first reported as promising, with $90-94 \%$ sensitivity and 77-95\% specificity. Additional validation studies however, yielded sensitivities between 38-90.5\% and specificities between 54-100\%, indicating that initial results might have been too optimistic (Supplementary Figure 3). Although optimism correction approaches have been described ${ }^{31}$, external validation studies, preferably using similar design and technical assays as the initial study, are necessary to assess the expected future range of sensitivities and specificities.

To ultimately draw conclusions on diagnostic biomarker performance, and increase the LoE to sufficient levels for clinical translation, large prospective clinical trials, or meta-analyses are necessary. ${ }^{24,26}$ However, due to variations in study design, patient selection, test characteristics and outcome assessment, meta-analysis is often not feasible. In addition, the quality and usefulness of meta-analyses completely depend on the input data quality, emphasizing the importance of high quality of individual studies and technical assays. 
Figure 2. Number of yearly publications for the tissue- (a) and body fluid-based (b) CRC DNA methylation biomarkers studied more than once

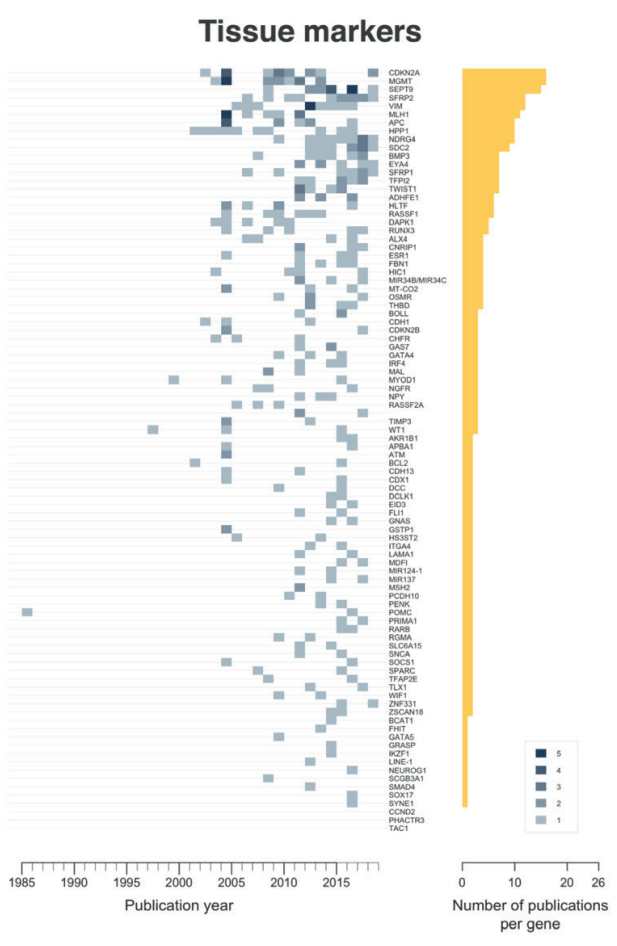

\section{Bodily fluid markers}

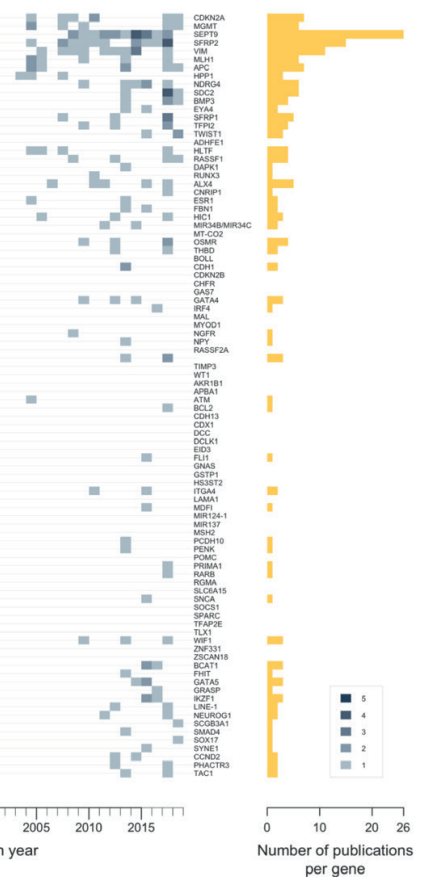

Number of yearly publications for the tissue- (a) and body fluid-based (b) colorectal cancer DNA methylation biomarkers studied more than once. Darker colors reflect a higher number of publications for the specified biomarker in a particular year. The gliding box indicates the publication count by color. The length of bars represents the total number of publications of the specified biomarker from 1985 to 2019. 
Many studies in this review used different biomarker assays; often it was unclear whether these measured the most relevant DNA methylation signal.

Also, suboptimal technical assays, poorly specific primers and suboptimal DNA methylation locations were used; therefore studies were not adding evidence on the potential diagnostic value of a marker.

\section{Overview of CRC biomarkers in bodily fluids}

Of 109 included studies on bodily fluids, 42 (38.5\%) focused on stool, 63 (57.8\%) on blood (plasma, serum, whole blood or cells derived from blood), one on urine $(0.9 \%)$, two on stool and blood $(1.8 \%)$ and one on blood, urine and stool $(0.9 \%)$. Study characteristics are shown in Supplementary Table 4.

Forest plots were constructed for 37 markers studied in more than two studies or populations (Supplementary Figure 3). Variations in sensitivity and specificity are probably partly due to reasons mentioned earlier (e.g. flaws in study design, lack of validation, initial optimism of results) but could also be caused by methodological differences between studies, reflected in patient or sample selection, technical assays, statistical approaches and varying sample sizes. This is e.g. shown for VIM methylation, with sensitivities between $<20 \%-80 \%$, and specificities $<90 \%$ but also for markers, such as APC, MLH1 and RASSF1A (Supplementary Figure 3).

\section{Comparison to currently implemented clinical tests}

From all biomarkers in this review, only a small, selected group holds sufficient potential to warrant further validation. However, it remains challenging to identify this group. An important issue is the additional value as compared to the current non-invasive test for early CRC detection (i.e. FIT). As FIT is already widely accepted and implemented, a biomarker, alone or in a panel, should add value or be more cost-effective than FIT, otherwise the chances of clinical translation will be small. For CRC, FIT has an estimated sensitivity between $68-87 \%$ and a specificity of $96-$ $98 \% .^{13,32}$ 
For advanced adenomas, sensitivity is substantially lower, $23-40 \%{ }^{10,33}$ leaving room for improvement. Most single markers in this review, performed less well for CRC (Supplementary Figure 3). From markers studied more than once, 17/37 (45.9\%; APC, MLH1, MGMT, OSMR, HIC1, HLTF, WIF1, BCAT1, ESR1, HPP1, IKZF1, ITGA4, LINE1, NEUROG1, PHACTR3, TAC1, THBD) did not report a sensitivity for CRC as good as FIT. Although specificity ranges appear to be more in line with FIT, performance is less for some markers (SFRP1, RASSF1A, CYCD2, ESR1, NEUROG1, TAC1). Even for established DNA methylation markers which are already used in clinical tests, (NDRG4 and BMP3 in Cologuard $\AA$ ) or have been launched as alternative for FIT (SEPTIN9 in EpiproColon/ColoVantage ${ }^{\circledR}$ and EpiproColon $\AA^{22}$ ), some published sensitivities or specificities are lower than those of FIT. The fact that these specific DNA methylation markers have incremental diagnostic value indicates that sensitivity and/or specificity alone should not be used to draw final conclusions on the diagnostic value of a marker. Instead, this conclusion might only be drawn after evaluating candidates, alone or in multi-marker panels, in predictive models also containing FIT.

\section{Quality of reporting}

The updated Standards for Reporting of Diagnostic Accuracy Studies (STARD) criteria were used to score included studies. ${ }^{24}$ STARD scores for the 109 included studies ranged from 3.5 to 21 points out of 22 points maximum (median 12.9 points) (Figure 3a). An overview of STARD item scores is shown in Supplementary Table 5. Items 3 (Intended biomarker use), 4 (Study objectives), 5 (Data collection) and 10 a (Assay method) were completely or partially described in over $90 \%$ of included studies, whereas items 15 (Handling of missing biomarker data) and 18 (Sample size/power) were reported in less than 10\% (Figure $3 b$ ). This shows that although STARD criteria are widely accepted, actual adherence to STARD is poor. 
However, STARD criteria might not be completely appropriate for biomarker studies. Consequently, several items could not be scored in this review. These include e.g. item 11 (Reference rationale) as the reference is the presence of disease and item 25 (Adverse events) as no adverse events are expected for biomarker analyses on bodily fluids. Other items might need more emphasis as these are essential for correct data interpretation, or experimental replication. These include detailed descriptions of all laboratory methods, not only the DNA methylation test (index test), and detailed descriptions of sample collection, processing and storage. Redefining current STARD criteria to better serve biomarker studies would improve uniform, high-quality reporting of these studies and facilitate meta-analyses.

Poor reporting is not synonymous to poor research or an useless biomarker, but it severely hampers validation studies and meta-analyses. As independent external validation is limited, the performance of large clinical trials is costly and complicated, and the inclusion of sufficient cases is challenging, systematic reviews and metaanalyses are necessary to increase the LoE for potential biomarkers so that they can be considered for clinical practice. However, for meta-analysis, high individual study quality is crucial.

\section{Problems in study design and methodology}

Problems previously described to hinder efficient clinical translation ${ }^{29,34-37}$ were also observed in this systematic review, including methodological heterogeneity and poor reporting quality (Supplementary Table 5). Additionally, other problems that have received less attention, were identified, such as technical heterogeneity. Most studies reported sensitivity and specificity, but ignored positive and negative predictive value (PPV and NPV), although these are important in clinical decision making for populations and individual patients.

Across studies, large sample size variations were observed; the smallest study counted five subjects and the largest 1315 (Supplementary Table 4), but sample 
Figure 3. Quality assessment (STARD) of 109 articles reporting body bodily fluid-based DNA methylation biomarker for CRC
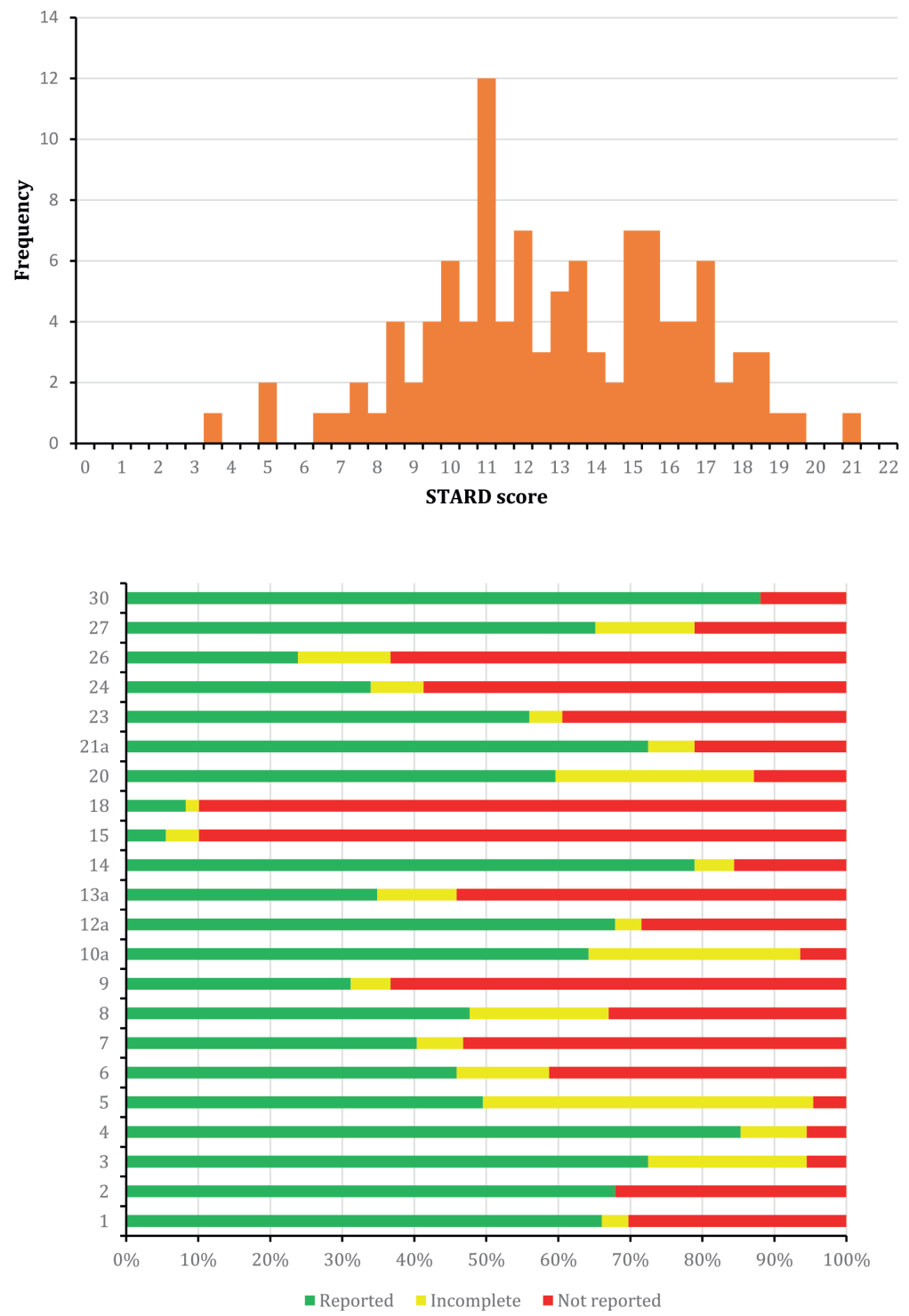

(a) Histogram depicting the STARD score distribution for body bodily fluid-based biomarker studies (mean STARD score $=12.9$, standard deviation $=3.5$ ).

(b) Stacked bar chart showing the percentage of completeness of each STARD item. 
size justification was lacking in 98/109 studies (89.9\%). Although small sample sizes are not synonymous for low quality, the risk of false-positive findings is significantly larger ${ }^{38}$, and multiple small studies should be combined to draw appropriate conclusions on actual biomarker value. However, due to large interstudy methodological heterogeneity, this is often impossible, resulting in a constantly low LoE (none of the included studies reached LoE I) for those markers without prospects to clinical translation (Supplementary Table 5). Moreover, even if studies could theoretically be combined, many likely suffer from (selection)bias, combining them would not yield a representative sample of the target population. Also, as primarily selected populations were used, with CRC patients and healthy controls already referred for colonoscopy, it can be assumed that biomarker sensitivity will probably decrease when applied to the general screening population. Specificity however, might actually be higher as compared to the study setting. ${ }^{39}$

Next to methodological heterogeneity, technical heterogeneity has similar consequences. Within 109 included studies, analytic methods could be categorized in 19 different approaches to measure methylation. Methylation-specific PCR (MSP) was most widely used (50 studies; $45.9 \%$ ), followed by quantitative-MSP (23 studies; 21.1\%). Especially if not optimized for the specific question, different technical assays can yield different results. ${ }^{40,41}$ Often, different experimental settings ${ }^{34,42,43}$ were used, including different DNA input and cut-offs to process quantitative results. Overall, 13 methods of cut-off determination for quantitative data were reported, but often without information on the rationale. This technical heterogeneity can hinder inter-study comparisons and consequently hamper improvement of LoE and clinical translation. Nevertheless, when carefully selected and optimized, different technical assays can yield comparable results. ${ }^{44}$ Different techniques can be used for validation purposes in that case, but it is essential that assays are reported properly and in great detail. 


\section{The way forward: How to bring diagnostic CRC DNA methylation markers to the clinic}

There are serious concerns about methodology and study design, which affect study quality. Poor reporting further complicates the assessment of results. Clinical usefulness of many diagnostic DNA methylation markers is questionable, because they were only investigated in tissue. Even when assessed in bodily fluids, comparisons to the standard method (FIT) are rare, hampering definitive conclusions on the clinical utility of DNA methylation markers (Figure 4).

Although extensively studied, only three DNA methylation markers $(0.8 \%)$ are currently used in clinical practice. Although explorative studies on DNA methylation markers in small populations without validation can be valuable for scientific reasons, research aiming for clinical translation clearly needs a new approach.

First, as summarized in the 2014 Lancet series on increasing value and reducing waste in biomedical research, biomarker development should start with a consensus statement that a true clinical need is present. ${ }^{34}$ This is an important responsibility of researchers, research funders and regulators ${ }^{45}$; new research should only be initiated when research questions cannot be answered satisfactorily with available evidence. ${ }^{46}$ The main goal should be to improve the LoE of candidate biomarkers, as this is necessary for clinical translation.

Second, the intended use of a biomarker should be clearly defined, as is also emphasized in guidelines (e.g. PRoBE design principles). ${ }^{43}$ Here, specimen and cohort choice plays an important role, especially in diseases such as CRC where only non-invasive diagnostic biomarkers are clinically relevant. To improve clinical practice, diagnostic biomarkers need to complement (or replace) existing diagnostic measures. Therefore, in CRC, comparison to FIT is a prerequisite. For this, definition of the performance measure is crucial and should be carefully considered. ${ }^{43,47}$ 
CHAPTER 2

Figure 4: Gap between CRC methylation marker research and clinical implementation
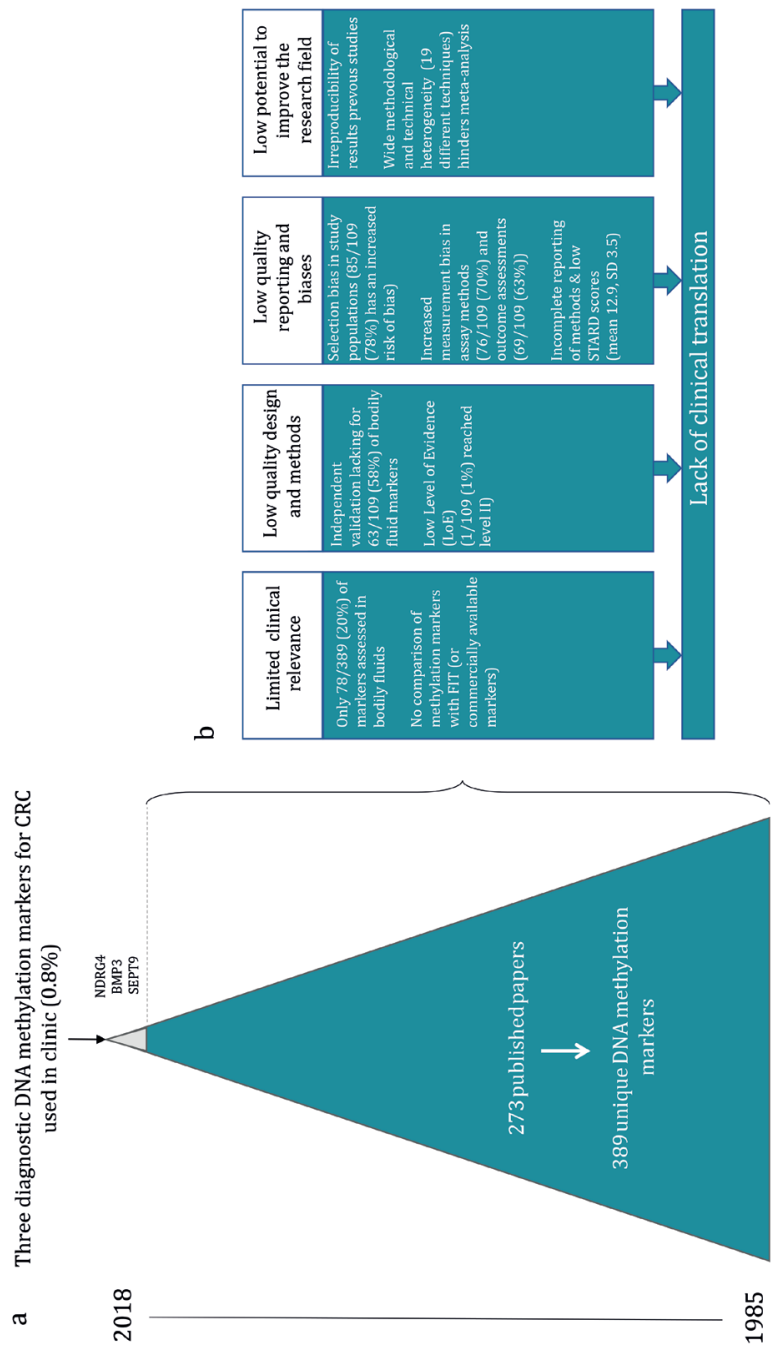

(a) The clinical translation rate of DNA methylation markers for early diagnosis of CRC (0.8\%) was calculated by comparing the number of commercially available biomarkers with the total number of published biomarkers (from 1985-2018).

(b) Four stages of waste in the development and reporting of DNA methylation marker research in CRC relevant to clinicians and patients (adapted from Avoidable waste in the production and reporting of research evidence. Lancet, Chalmers and Glasziou, 2009. 
Last, reported data should support the claims on the diagnostic potential of a marker, and should be adequately reported to facilitate independent validation. This includes an adjustment of the reporting guidelines for biomarker studies and strict implementation by scientific journals.

Although the Lancet series on increasing value and reducing waste in biomedical research dates from 2014, there has unfortunately been little change in the DNA methylation marker field to avoid research waste. Although the drawbacks as stated in our review are applicable to diagnostic DNA methylation markers for CRC, they also apply to the broader biomarker research field. To stop this research waste in biomarker research, we need to raise the methodological bar, and take into account some important considerations prior to designing biomarker studies, in order to 1) improve reporting quality, 2) facilitate external validation, 3) facilitate metaanalyses, and 4) improve LoE (Figure 5). Only then we will be able to identify and develop biomarkers that can make a difference for patients.

Figure 5: Recommendations for future DNA methylation biomarker studies

\begin{tabular}{|ll|}
\hline General \\
\hline - & Update reporting guidelines specifically for biomarker studies \\
\hline Prior to study design \\
\hline - & Consensus statement clinical need \\
\hline - & Define intended biomarker use \\
\hline - & Evaluate available evidence \& current LoE \\
\hline Define study design to complement available evidence and add to LoE \\
\hline - & $\begin{array}{l}\text { Define specimen and cohort to complement available evidence and } \\
\text { facilitate external validation or meta-analyses }\end{array}$ \\
\hline Define technical method to facilitate external validation and meta-analyses \\
\hline During/after study \\
\hline$\bullet$ & $\begin{array}{l}\text { Compare results to golden standard methods (e.g. FIT) to complement } \\
\text { available evidence }\end{array}$ \\
\hline - & $\begin{array}{l}\text { Adhere to reporting guidelines to facilitate external validation or } \\
\text { meta-analyses }\end{array}$ \\
\hline
\end{tabular}




\section{References}

1. Edwards BK, et al. Annual report to the nation on the status of cancer, 1975-2006, featuring colorectal cancer trends and impact of interventions (risk factors, screening, and treatment) to reduce future rates. Cancer 2010;116(3):544-73.

2. Bray F, et al. Global cancer statistics 2018: GLOBOCAN estimates of incidence and mortality worldwide for 36 cancers in 185 countries. CA Cancer J Clin 2018;68(6):394-424.

3. Arnold M, et al. Global patterns and trends in colorectal cancer incidence and mortality. Gut 2017;66(4):68391.

4. Bradley CJ, et al. Productivity savings from colorectal cancer prevention and control strategies. Am J Prev Med 2011;41(2):e5-e14.

5. Greuter MJ, et al. Long-Term Impact of the Dutch Colorectal Cancer Screening Program on Cancer Incidence and Mortality-Model-Based Exploration of the Serrated Pathway. Cancer Epidemiol Biomarkers Prev 2016;25(1):135-44.

6. Navarro M, et al. Colorectal cancer population screening programs worldwide in 2016: An update. World J Gastroenterol 2017;23(20):3632-42.

7. Denters MJ, et al. Patient burden of colonoscopy after positive fecal immunochemical testing for colorectal cancer screening. Endoscopy 2013;45(5):342-9.
8. Reumkens A, et al. Post-Colonoscopy Complications: A Systematic Review, Time Trends, and Meta-Analysis of Population-Based Studies. Am J Gastroenterol 2016;111(8):1092-101.

9. van Lanschot MC, et al. Molecular stool testing as an alternative for surveillance colonoscopy: a crosssectional cohort study. BMC Cancer 2017;17(1):116.

10. Toes-Zoutendijk E, et al. Real-Time Monitoring of Results During First Year of Dutch Colorectal Cancer Screening Program and Optimization by Altering Fecal Immunochemical Test CutOff Levels. Gastroenterology 2017;152(4):767-75 e2.

11. Lieberman D. Colon-polyp surveillance--do patients benefit? N Engl J Med 2014;371(9):860-1.

12. Stracci F, et al. Colorectal cancer screening: tests, strategies, and perspectives. Front Public Health 2014;2:210.

13. Gies A, et al. Direct Comparison of Diagnostic Performance of 9 Quantitative Fecal Immunochemical Tests for Colorectal Cancer Screening. Gastroenterology 2018;154(1):93-104. 
14. van Rossum LG, et al. Cutoff value determines the performance of a semi-quantitative immunochemical faecal occult blood test in a colorectal cancer screening programme. $\mathrm{Br}$ J Cancer 2009;101(8):1274-81.

15. Silva TD, et al. DNA methylation as an epigenetic biomarker in colorectal cancer. Oncol Lett 2013;6(6):1687-92.

16. Toiyama Y, et al. DNA methylation and microRNA biomarkers for noninvasive detection of gastric and colorectal cancer. Biochem Biophys Res Commun 2014;455(1-2):43-57.

17. Rasmussen SL, et al. Hypermethylated DNA, a circulating biomarker for colorectal cancer detection. PLoS One 2017;12(7):e0180809.

18. Ma Z, et al. Roles of Methylated DNA Biomarkers in Patients with Colorectal Cancer. Dis Markers 2019;2019:2673543.

19. Hong L, Ahuja N. DNA methylation biomarkers of stool and blood for early detection of colon cancer. Genet Test Mol Biomarkers 2013;17(5):401-6.

20. Muller HM, et al. Methylation changes in faecal DNA: a marker for colorectal cancer screening? Lancet 2004;363(9417):1283-5.
21. Melotte V, et al. N-Myc downstreamregulated gene 4 (NDRG4): a candidate tumor suppressor gene and potential biomarker for colorectal cancer. J Natl Cancer Inst 2009;101(13):916-27.

22. Koch A, et al. Analysis of DNA methylation in cancer: location revisited. Nat Rev Clin Oncol 2018;15(7):459-66.

23. McInnes MDF, et al. Preferred Reporting Items for a Systematic Review and Meta-analysis of Diagnostic Test Accuracy Studies: The PRISMA-DTA Statement. JAMA 2018;319(4):388-96.

24. Bossuyt PM, et al. STARD 2015: an updated list of essential items for reporting diagnostic accuracy studies. BMJ 2015;351:h5527.

25. Cohen JF, et al. STARD 2015 guidelines for reporting diagnostic accuracy studies: explanation and elaboration. BMJ Open 2016;6(11):e012799.

26. Howick J, et al. Oxford Centre for Evidence-Based Medicine 2011 Levels of Evidence. 2011.

27. Poste G, et al. The national biomarker development alliance: confronting the poor productivity of biomarker research and development. Expert Rev Mol Diagn 2015;15(2):211-8. 
28. Steyerberg EW, Harrell FE, Jr. Prediction models need appropriate internal, internal-external, and external validation. J Clin Epidemiol 2016;69:245-7.

29. Ioannidis JPA, Bossuyt PMM. Waste, Leaks, and Failures in the Biomarker Pipeline. Clin Chem 2017;63(5):96372.

30. Steyerberg EW, et al. Prognosis Research Strategy (PROGRESS) 3: prognostic model research. PLoS Med 2013;10(2):e1001381

31. Harrell F. Regression Modeling Strategies, With Applications to Linear Models, Logistic and Ordinal Regression, and Survival Analysis. 2 ed: Springer International Publishing; 2015.

32. Oort FA, et al. Colonoscopycontrolled intra-individual comparisons to screen relevant neoplasia: faecal immunochemical test vs. guaiac-based faecal occult blood test. Aliment Pharmacol Ther 2010;31(3):432-9.

33. Imperiale TF, et al. Performance Characteristics of Fecal Immunochemical Tests for Colorectal Cancer and Advanced Adenomatous Polyps: A Systematic Review and Meta-analysis. Ann Intern Med 2019.

34. Ioannidis JP, et al. Increasing value and reducing waste in research design, conduct, and analysis. Lancet 2014;383(9912):166-75
35. Macleod MR, et al. Biomedical research: increasing value, reducing waste. Lancet 2014;383(9912):1014.

36. Pepe MS. The statistical evaluation of medical tests for classification and prediction. Oxford University Press; 2004

37. Maxim LD, et al. Screening tests: a review with examples. Inhal Toxicol 2014;26(13):811-28.

38. Ioannidis JP. Why most published research findings are false. PLoS Med 2005;2(8):e124.

39. Usher-Smith JA, et al. The spectrum effect in tests for risk prediction, screening, and diagnosis. BMJ 2016;353:i3139.

40. Claus $\mathrm{R}$, et al. A systematic comparison of quantitative highresolution DNA methylation analysis and methylation-specific PCR. Epigenetics 2012;7(7):772-80.

41. Quillien V, et al. Comparative assessment of 5 methods (methylation-specific polymerase chain reaction, MethyLight, pyrosequencing, methylation-sensitive high-resolution melting, and immunohistochemistry) to analyze 06-methylguanine-DNA-methyltranferase in a series of 100 glioblastoma patients. Cancer 2012;118(17):4201-11. 
42. Bustin SA, et al. The MIQE guidelines: minimum information for publication of quantitative real-time PCR experiments. Clin Chem 2009;55(4):611-22.

43. Pepe MS, et al. Improving the quality of biomarker discovery research: the right samples and enough of them. Cancer Epidemiol Biomarkers Prev 2015;24(6):944-50.

44. Blueprint.consortium. Quantitative comparison of DNA methylation assays for biomarker development and clinical applications. Nat Biotechnol 2016;34(7):726-37.

45. Chalmers I, et al. How to increase value and reduce waste when research priorities are set. Lancet 2014;383(9912):156-65.

46. Chalmers I, Glasziou P. Avoidable waste in the production and reporting of research evidence. Lancet 2009;374(9683):86-9.

47. Young GP, et al. Recommendations for a step-wise comparative approach to the evaluation of new screening tests for colorectal cancer. Cancer 2016;122(6):826-39. 


\section{Supplementary material}

Supplementary Figure 1. Flowchart of the study identification process
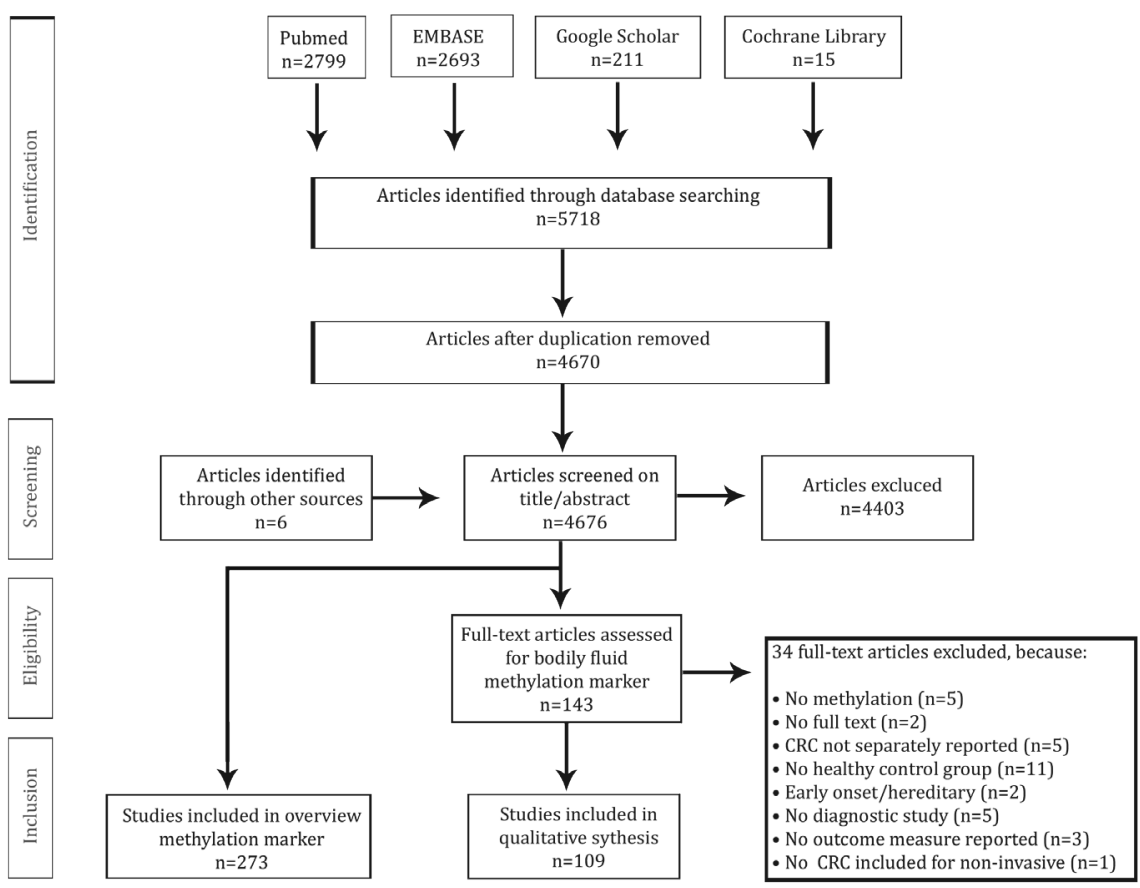

A total of 109 articles reporting body fluid-based colorectal cancer DNA methylation markers were selected for qualitative assessment 
Supplementary Figure 2. Venn diagram of studies and markers

\section{Article overview}

Among 273 articles, there were 164 articles focusing on tissue, 71 articles on bodily fluid samples and 38 studies using both tissue and bodily fluid samples

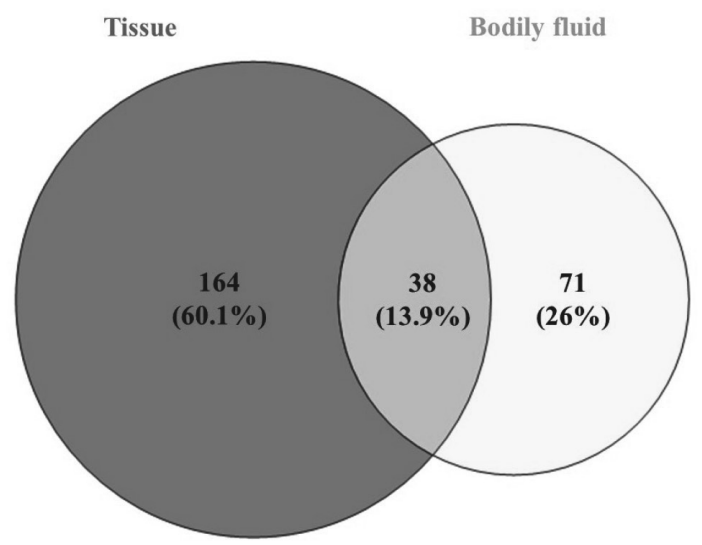

\section{Marker overview}

274 articles generated 389 biomarkers, 301 markers studied in tissue, 18 markers in bodily fluid samples and 70 markers in both tissue and bodily fluid samples
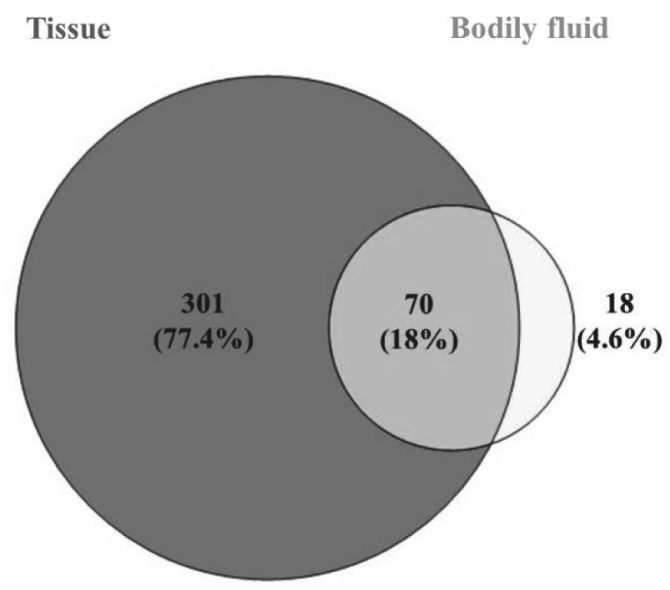
Supplementary Figure 3. Forest plots of reported DNA methylation markers in colorectal cancer studies
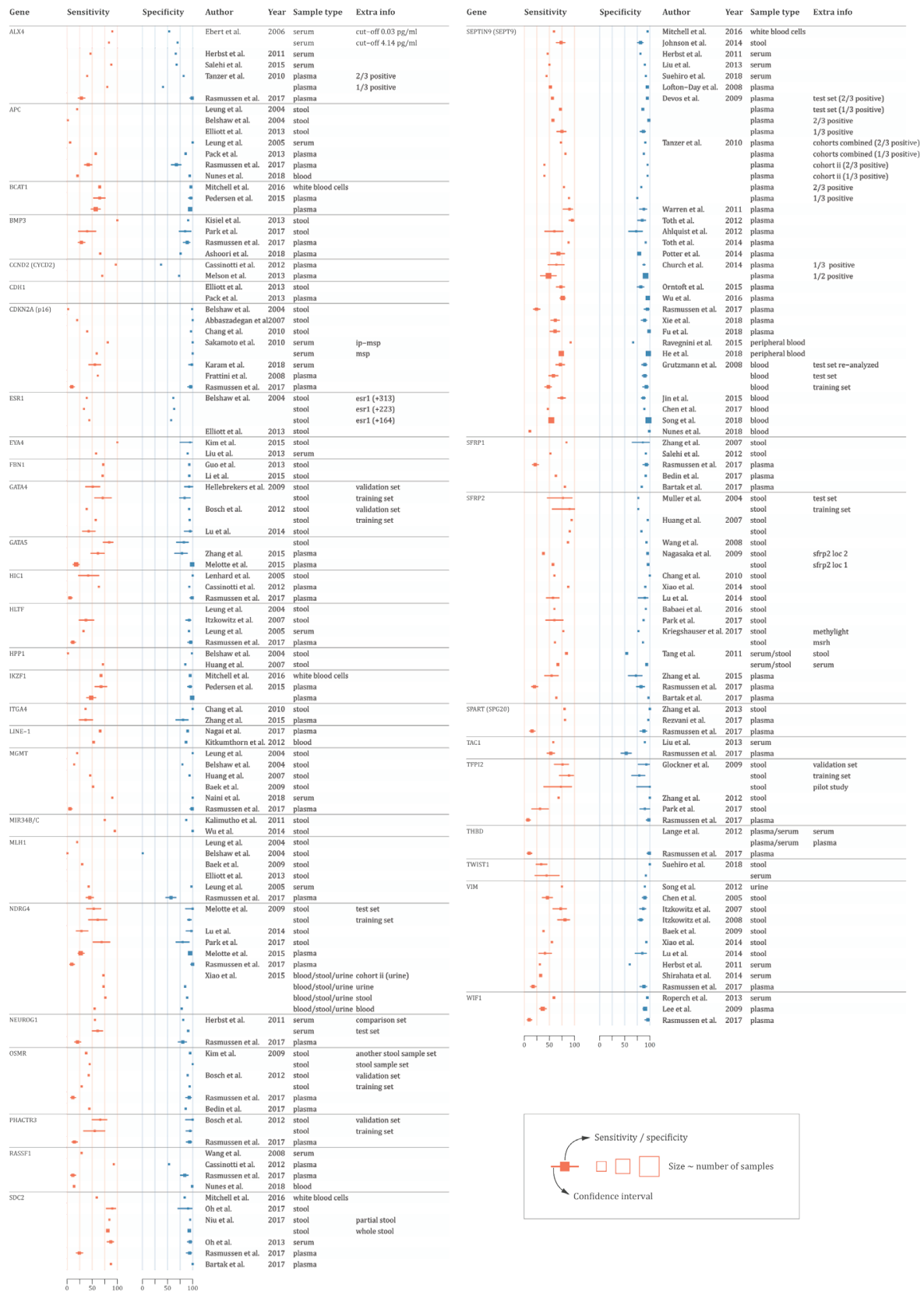
Supplementary Table 1. Search strategy for diagnostic methylation markers in colorectal cancer

\begin{tabular}{|c|c|c|c|c|}
\hline & Pubmed & Embase & Cochrane Library & Google Scholar \\
\hline 1 & $\begin{array}{l}\text { "methylation"[MeSH Terms] OR } \\
\text { "methylation"[All Fields] OR } \\
\text { hypermethylation[All Fields] OR } \\
\text { hypomethylation[All Fields] OR } \\
\text { promoter[All Fields] }\end{array}$ & $\begin{array}{l}\text { 'DNA methylation' OR } \\
\text { 'hypermethylation' OR } \\
\text { 'hypomethylation' OR } \\
\text { 'methylation' OR 'pro- } \\
\text { moter methylation' }\end{array}$ & $\begin{array}{l}\text { 'methylation' } \\
\text { [MeSH Terms] }\end{array}$ & 'DNA methylation' \\
\hline 2 & $\begin{array}{l}\text { “colorectal neoplasms"[MeSH } \\
\text { Terms] OR (("colorectal”[All } \\
\text { Fields] OR "rectal"[All Fields] OR } \\
\text { "colon"[All Fields] OR “colonic"[All } \\
\text { Fields] OR “sigmoid”[All Fields]) } \\
\text { AND ("carcinoma"[All Fields] OR } \\
\text { "cancer"[All Fields] OR "neo- } \\
\text { plasm"[All Fields] OR “tumor"[All } \\
\text { Fields])) }\end{array}$ & $\begin{array}{l}\text { 'colorectal cancer' OR } \\
\text { 'colon cancer' OR 'rectal } \\
\text { cancer' }\end{array}$ & $\begin{array}{l}\text { 'colorectal } \\
\text { neoplasms' [MeSH } \\
\text { Terms] }\end{array}$ & 'colorectal cancer' \\
\hline 3 & $\begin{array}{l}\text { "diagnosis"[MeSH Terms] OR “diag- } \\
\text { nosis"[All Fields] OR “detection" [All } \\
\text { Fields] }\end{array}$ & $\begin{array}{l}\text { 'diagnosis' OR 'detec- } \\
\text { tion' }\end{array}$ & $\begin{array}{l}\text { 'diagnosis' [MeSH } \\
\text { Terms] }\end{array}$ & 'diagnosis' \\
\hline \multicolumn{5}{|l|}{4} \\
\hline & & & & 'clinical test' \\
\hline Search & 1 and 2 and 3 & 1 and 2 and 3 & 1 and 2 and 3 & $\begin{array}{l}1 \text { and } 2 \text { and } 3 \\
\text { and } 4\end{array}$ \\
\hline
\end{tabular}


Supplementary Table 2. STARD checklist, adapted for this study

\begin{tabular}{|c|c|c|c|}
\hline Section & No & Item & Interpretation and scoring \\
\hline \multicolumn{4}{|c|}{ Title or abstract } \\
\hline & 1 & $\begin{array}{l}\text { Identification as a study of diagnostic accuracy using at } \\
\text { least one measure of accuracy (such as sensitivity, } \\
\text { specificity, predictive values, or AUC) }\end{array}$ & \\
\hline \multicolumn{4}{|l|}{ Abstract } \\
\hline & 2 & $\begin{array}{l}\text { Structured summary of study design, methods, results, and } \\
\text { conclusions (for specific guidance, see STARD for Abstracts) }\end{array}$ & \\
\hline \multicolumn{4}{|l|}{ Introduction } \\
\hline & 3 & $\begin{array}{l}\text { Scientific and clinical background, including the intended } \\
\text { use and clinical role of the index test }\end{array}$ & $\begin{array}{l}\text { Intended use or clinical role } \\
\text { not mentioned }=0.5\end{array}$ \\
\hline & 4 & Study objectives and hypotheses & $\begin{array}{l}\text { Also } 1.0 \text { point if only objec- } \\
\text { tives are mentioned }\end{array}$ \\
\hline \multicolumn{4}{|l|}{ Methods } \\
\hline $\begin{array}{l}\text { Study } \\
\text { design }\end{array}$ & 5 & $\begin{array}{l}\text { Whether data collection was planned before the index } \\
\text { test and reference standard were performed (prospective } \\
\text { study) or after (retrospective study) }\end{array}$ & $\begin{array}{l}\text { If described: } 1.0 \text { point, if you } \\
\text { can implicitly conclude it from } \\
\text { text: } 0.5 \text { point }\end{array}$ \\
\hline \multirow[t]{4}{*}{ Participants } & 6 & Eligibility criteria & \\
\hline & 7 & $\begin{array}{l}\text { On what basis potentially eligible participants were } \\
\text { identified (such as symptoms, results from previous tests, } \\
\text { inclusion in registry) }\end{array}$ & \\
\hline & 8 & $\begin{array}{l}\text { Where and when potentially eligible participants were } \\
\text { identified (setting, location, and dates) }\end{array}$ & \\
\hline & 9 & $\begin{array}{l}\text { Whether participants formed a consecutive, random, or } \\
\text { convenience series }\end{array}$ & \\
\hline \multirow[t]{7}{*}{$\begin{array}{l}\text { Test } \\
\text { methods }\end{array}$} & $10 \mathrm{a}$ & Index test, in sufficient detail to allow replication & $\begin{array}{l}\text { In case the article referred to } \\
\text { another article for primer and } \\
\text { probe sequences, } 0.5 \text { point } \\
\text { was awarded. }\end{array}$ \\
\hline & $10 \mathrm{~b}$ & Reference standard, in sufficient detail to allow replication & $\begin{array}{l}\text { Excluded. Not applicable to } \\
\text { biomarker studies. }\end{array}$ \\
\hline & 11 & $\begin{array}{l}\text { Rationale for choosing the reference standard (if } \\
\text { alternatives exist) }\end{array}$ & $\begin{array}{l}\text { Excluded. Not applicable to } \\
\text { biomarker studies. }\end{array}$ \\
\hline & $12 \mathrm{a}$ & $\begin{array}{l}\text { Definition of and rationale for test positivity cut-offs or } \\
\text { result categories of the index test, distinguishing pre- } \\
\text { specified from exploratory }\end{array}$ & $\begin{array}{l}\text { (Q-MSP should usually define } \\
\text { cut-off) }\end{array}$ \\
\hline & $12 \mathrm{~b}$ & $\begin{array}{l}\text { Definition of and rationale for test positivity cut-offs or } \\
\text { result categories of the reference standard, distinguishing } \\
\text { pre-specified from exploratory }\end{array}$ & $\begin{array}{l}\text { Excluded. Not applicable to } \\
\text { biomarker studies. }\end{array}$ \\
\hline & $13 a$ & $\begin{array}{l}\text { Whether clinical information and reference standard } \\
\text { results were available to the performers or readers of the } \\
\text { index test }\end{array}$ & \\
\hline & $13 b$ & $\begin{array}{l}\text { Whether clinical information and index test results were } \\
\text { available to the assessors of the reference standard }\end{array}$ & $\begin{array}{l}\text { Excluded. Not applicable to } \\
\text { biomarker studies. }\end{array}$ \\
\hline
\end{tabular}




\begin{tabular}{|c|c|c|c|}
\hline Section & No & Item & Interpretation and scoring \\
\hline \multicolumn{4}{|l|}{ Methods } \\
\hline \multirow[t]{5}{*}{ Analysis } & 14 & $\begin{array}{l}\text { Methods for estimating or comparing measures } \\
\text { of diagnostic accuracy }\end{array}$ & $\begin{array}{l}\text { Only frequencies }=0.5 \text { points, if sens }+ \\
\text { spec are mentioned }=1.0 \text { point. }\end{array}$ \\
\hline & 15 & $\begin{array}{l}\text { How indeterminate index test or reference } \\
\text { standard results were handled }\end{array}$ & \\
\hline & 16 & $\begin{array}{l}\text { How missing data on the index test and } \\
\text { reference standard were handled }\end{array}$ & $\begin{array}{l}\text { Excluded. Not applicable to biomarker } \\
\text { studies. }\end{array}$ \\
\hline & 17 & $\begin{array}{l}\text { Any analyses of variability in diagnostic } \\
\text { accuracy, distinguishing pre-specified from } \\
\text { exploratory }\end{array}$ & $\begin{array}{l}\text { Excluded. Not applicable to biomarker } \\
\text { studies. }\end{array}$ \\
\hline & 18 & $\begin{array}{l}\text { Intended sample size and how it was } \\
\text { determined }\end{array}$ & \\
\hline \multicolumn{4}{|l|}{ Results } \\
\hline \multirow[t]{5}{*}{ Participants } & 19 & Flow of participants, using a diagram & $\begin{array}{l}\text { Excluded. Not applicable to biomarker } \\
\text { studies }\end{array}$ \\
\hline & 20 & $\begin{array}{l}\text { Baseline demographic and clinical } \\
\text { characteristics of participants }\end{array}$ & $\begin{array}{l}\text { If characteristics are not summarized, } \\
\text { thus individual patient samples are de- } \\
\text { scribed (often in supplement) } 0.5 \text { points. }\end{array}$ \\
\hline & $21 \mathrm{a}$ & $\begin{array}{l}\text { Distribution of severity of disease in those with } \\
\text { the target condition }\end{array}$ & \\
\hline & $21 b$ & $\begin{array}{l}\text { Distribution of alternative diagnoses in those } \\
\text { without the target condition }\end{array}$ & $\begin{array}{l}\text { Excluded. Not applicable to biomarker } \\
\text { studies. }\end{array}$ \\
\hline & 22 & $\begin{array}{l}\text { Time interval and any clinical interventions } \\
\text { between index test and reference standard }\end{array}$ & $\begin{array}{l}\text { Excluded. Not applicable to biomarker } \\
\text { studies. }\end{array}$ \\
\hline \multirow[t]{3}{*}{ Test results } & 23 & $\begin{array}{l}\text { Cross tabulation of the index test results (or } \\
\text { their distribution) by the results of the } \\
\text { reference standard }\end{array}$ & $\begin{array}{l}\text { Cross tabulation or distribution of } \\
\text { methylation marker for healthy } / \mathrm{CRC}= \\
1.0 \text { point }\end{array}$ \\
\hline & 24 & $\begin{array}{l}\text { Estimates of diagnostic accuracy and their } \\
\text { precision (such as 95\% confidence intervals) }\end{array}$ & $\begin{array}{l}\text { Precision for estimates of diagnostic accu- } \\
\text { racy (such as } 95 \% \text { confidence intervals) } \\
\text { are reported }=1.0 \text { point - only frequencies } \\
\text { with CI/SD/SEM }=0.5 \text { points - no CI's }=0\end{array}$ \\
\hline & 25 & $\begin{array}{l}\text { Any adverse events from performing the index } \\
\text { test or the reference standard }\end{array}$ & $\begin{array}{l}\text { Excluded. Not applicable to biomarker } \\
\text { studies. }\end{array}$ \\
\hline \multicolumn{4}{|l|}{ Discussion } \\
\hline & 25 & $\begin{array}{l}\text { Any adverse events from performing the index } \\
\text { test or the reference standard }\end{array}$ & $\begin{array}{l}\text { Excluded. Not applicable to biomarker } \\
\text { studies. }\end{array}$ \\
\hline & 26 & $\begin{array}{l}\text { Study limitations, including sources of potential } \\
\text { bias, statistical uncertainty, and generalisability }\end{array}$ & $\begin{array}{l}\text { In case only one limitation was mentioned } \\
\text { or limitations were mentioned without } \\
\text { explanation, } 0.5 \text { point was awarded. } \\
\text { No limitations mentioned }=0 \text { point }\end{array}$ \\
\hline & 27 & $\begin{array}{l}\text { Implications for practice, including the intended } \\
\text { use and clinical role of the index test }\end{array}$ & \\
\hline \multicolumn{4}{|c|}{ Other information } \\
\hline & 28 & Registration number and name of registry & $\begin{array}{l}\text { Excluded. Not applicable to biomarker } \\
\text { studies. }\end{array}$ \\
\hline & 29 & Where the full study protocol can be accessed & $\begin{array}{l}\text { Excluded. Not applicable to biomarker } \\
\text { studies. }\end{array}$ \\
\hline & 30 & $\begin{array}{l}\text { Sources of funding and other support; role of } \\
\text { funders }\end{array}$ & \\
\hline
\end{tabular}




\section{Supplementary Table 3. Included references}

\begin{tabular}{|c|c|}
\hline 1. & $\begin{array}{l}\text { Abbaszadegan M.R., Tavasoli A., Velayati A., et al. Stool-based DNA testing, a new noninvasive method for } \\
\text { colorectal cancer screening, the first report from Iran. World J Gastroenterol 2007, 13(10):1528-1533. }\end{array}$ \\
\hline 2. & $\begin{array}{l}\text { Ahlquist D.A., Taylor W.R., Mahoney D.W., et al. The stool DNA test is more accurate than the plasma septin } \\
9 \text { test in detecting colorectal neoplasia. Clin Gastroenterol Hepatol 2012, 10(3):272-277 e271. }\end{array}$ \\
\hline 3. & $\begin{array}{l}\text { Alizadeh Naini M., Kavousipour S., Hasanzarini M., et al. O6-Methyguanine-DNA Methyl Transferase } \\
\text { (MGMT) Promoter Methylation in Serum DNA of Iranian Patients with Colorectal Cancer. Asian Pacific } \\
\text { journal of cancer prevention : APJCP 2018, 19(5):1223-1227. }\end{array}$ \\
\hline 4. & $\begin{array}{l}\text { Ashoori H., Ghamarchehreh M.E., Tavallaei M., et al. Evaluation of the epigenetic biomarker bone morpho- } \\
\text { genic protein } 3 \text { for colorectal cancer diagnosis. Journal of Clinical and Diagnostic Research 2018, 12(11). }\end{array}$ \\
\hline 5. & $\begin{array}{l}\text { Babaei H., Mohammadi M., Salehi R. DNA methylation analysis of secreted frizzled-related protein } 2 \text { gene } \\
\text { for the early detection of colorectal cancer in fecal DNA. Niger Med J 2016, 57(4):242-245. }\end{array}$ \\
\hline 6. & $\begin{array}{l}\text { Baek Y.H., Chang E., Kim Y.J., et al. Stool methylation-specific polymerase chain reaction assay for the } \\
\text { detection of colorectal neoplasia in Korean patients. Dis Colon Rectum 2009, 52 (8):1452-1459; discussion } \\
\text { 1459-1463. }\end{array}$ \\
\hline 7. & $\begin{array}{l}\text { Bartak B.K., Kalmar A., Galamb O., et al. Blood Collection and Cell-Free DNA Isolation Methods Influence } \\
\text { the Sensitivity of Liquid Biopsy Analysis for Colorectal Cancer Detection. Pathol Oncol Res } 2018 .\end{array}$ \\
\hline 8. & $\begin{array}{l}\text { Bedin C., Enzo M.V., Del Bianco P., et al. Diagnostic and prognostic role of cell-free DNA testing for colorec- } \\
\text { tal cancer patients. Int J Cancer 2017, 140(8):1888-1898. }\end{array}$ \\
\hline 9. & $\begin{array}{l}\text { Belshaw N.J., Elliott G.O., Williams E.A., et al. Use of DNA from human stools to detect aberrant CpG } \\
\text { island methylation of genes implicated in colorectal cancer. Cancer Epidemiol Biomarkers Prev 2004, } \\
\text { 13(9):1495-1501. }\end{array}$ \\
\hline 10. & $\begin{array}{l}\text { Bosch L.J., Oort F.A., Neerincx M., et al. DNA methylation of phosphatase and actin regulator } 3 \text { detects } \\
\text { colorectal cancer in stool and complements FIT. Cancer Prev Res (Phila) 2012, 5(3):464-472. }\end{array}$ \\
\hline 11. & $\begin{array}{l}\text { Cassinotti E., Melson J., Liggett T., et al. DNA methylation patterns in blood of patients with colorectal } \\
\text { cancer and adenomatous colorectal polyps. Int J Cancer 2012, 131(5):1153-1157. }\end{array}$ \\
\hline 12. & $\begin{array}{l}\text { Chang E., Park D.I., Kim Y.J., et al. Detection of colorectal neoplasm using promoter methylation of ITGA4, } \\
\text { SFRP2, and p16 in stool samples: a preliminary report in Korean patients. Hepatogastroenterology 2010, } \\
57(101): 720-727 .\end{array}$ \\
\hline 13. & $\begin{array}{l}\text { Chen C.H., Yan S.L., Yang T.H., et al. The Relationship between the Methylated Septin-9 DNA Blood Test and } \\
\text { Stool Occult Blood Test for Diagnosing Colorectal Cancer in Taiwanese People. J Clin Lab Anal 2017, 31(1). }\end{array}$ \\
\hline 14. & $\begin{array}{l}\text { Chen W.D., Han Z.J., Skoletsky J., et al. Detection in fecal DNA of colon cancer-specific methylation of the } \\
\text { nonexpressed vimentin gene. J Natl Cancer Inst 2005, 97(15):1124-1132. }\end{array}$ \\
\hline 15. & $\begin{array}{l}\text { Church T.R., Wandell M., Lofton-Day C., et al. Prospective evaluation of methylated SEPT9 in plasma for } \\
\text { detection of asymptomatic colorectal cancer. Gut } 2014,63(2): 317-325 .\end{array}$ \\
\hline 16. & $\begin{array}{l}\text { deVos T., Tetzner R., Model F., et al. Circulating methylated SEPT9 DNA in plasma is a biomarker for } \\
\text { colorectal cancer. Clin Chem 2009, 55(7):1337-1346. }\end{array}$ \\
\hline 17. & $\begin{array}{l}\text { Ebert M.P., Model F., Mooney S., et al. Aristaless-like homeobox- } 4 \text { gene methylation is a potential marker } \\
\text { for colorectal adenocarcinomas. Gastroenterology 2006, 131(5):1418-1430. }\end{array}$ \\
\hline 18. & $\begin{array}{l}\text { Elliott G.O., Johnson I.T., Scarll J., et al. Quantitative profiling of CpG island methylation in human stool for } \\
\text { colorectal cancer detection. Int J Colorectal Dis 2013, 28(1):35-42. }\end{array}$ \\
\hline 19. & $\begin{array}{l}\text { Frattini M., Gallino G., Signoroni S., et al. Quantitative and qualitative characterization of plasma DNA } \\
\text { identifies primary and recurrent colorectal cancer. Cancer Letters 2008, 263(2):170-181. }\end{array}$ \\
\hline 20. & $\begin{array}{l}\text { Fu B., Yan P., Zhang S., et al. Cell-Free Circulating Methylated SEPT9 for Noninvasive Diagnosis and Moni- } \\
\text { toring of Colorectal Cancer. Dis Markers 2018, 2018:6437104. }\end{array}$ \\
\hline 21. & $\begin{array}{l}\text { Gao Y. Leukocyte DNA methylation and colorectal cancer among male smokers. World Journal of Gastroin- } \\
\text { testinal Oncology 2012, } 4(8) \text {. }\end{array}$ \\
\hline
\end{tabular}




\begin{tabular}{|c|c|}
\hline 22. & $\begin{array}{l}\text { Glockner S.C., Dhir M., Yi J.M., et al. Methylation of TFPI2 in stool DNA: a potential novel biomarker for the } \\
\text { detection of colorectal cancer. Cancer Res 2009, 69(11):4691-4699. }\end{array}$ \\
\hline 23. & $\begin{array}{l}\text { Guo Q., Song Y., Zhang H., et al. Detection of hypermethylated fibrillin-1 in the stool samples of colorectal } \\
\text { cancer patients. Med Oncol 2013, 30(4):695. }\end{array}$ \\
\hline 24. & $\begin{array}{l}\text { He C.G., Huang Q.Y., Chen L.S., et al. p33(ING1b) methylation in fecal DNA as a molecular screening tool for } \\
\text { colorectal cancer and precancerous lesions. Oncol Lett 2014, 7(5):1639-1644. }\end{array}$ \\
\hline 25. & $\begin{array}{l}\text { He N., Song L., Kang Q., et al. The Pathological Features of Colorectal Cancer Determine the Detection Per- } \\
\text { formance on Blood ctDNA. Technology in cancer research \& treatment 2018, 17:1533033818791794. }\end{array}$ \\
\hline 26. & $\begin{array}{l}\text { Hellebrekers D.M., Lentjes M.H., van den Bosch S.M., et al. GATA4 and GATA5 are potential tumor suppres- } \\
\text { sors and biomarkers in colorectal cancer. Clin Cancer Res 2009, 15(12):3990-3997. }\end{array}$ \\
\hline 27. & $\begin{array}{l}\text { Herbst A., Rahmig K., Stieber P., et al. Methylation of NEUROG1 in serum is a sensitive marker for the } \\
\text { detection of early colorectal cancer. Am J Gastroenterol 2011,106(6):1110-1118. }\end{array}$ \\
\hline 28. & $\begin{array}{l}\text { Huang Z., Li L., Wang J. Hypermethylation of SFRP2 as a potential marker for stool-based detection of } \\
\text { colorectal cancer and precancerous lesions. Dig Dis Sci 2007, 52(9):2287-2291. }\end{array}$ \\
\hline 29. & $\begin{array}{l}\text { Huang Z.H., Li L.H., Yang F., et al. Detection of aberrant methylation in fecal DNA as a molecular screening } \\
\text { tool for colorectal cancer and precancerous lesions. World J Gastroenterol 2007, 13(6):950-954. }\end{array}$ \\
\hline 30. & $\begin{array}{l}\text { Itzkowitz S., Brand R., Jandorf L., et al. A simplified, noninvasive stool DNA test for colorectal cancer detec- } \\
\text { tion. Am J Gastroenterol 2008, 103(11):2862-2870. }\end{array}$ \\
\hline 31. & $\begin{array}{l}\text { Itzkowitz S.H., Jandorf L., Brand R., et al. Improved fecal DNA test for colorectal cancer screening. Clin } \\
\text { Gastroenterol Hepatol 2007, 5(1):111-117. }\end{array}$ \\
\hline 32. & $\begin{array}{l}\text { Jin P., Kang Q., Wang X., et al. Performance of a second-generation methylated SEPT9 test in detecting } \\
\text { colorectal neoplasm. J Gastroenterol Hepatol 2015, 30(5):830-833. }\end{array}$ \\
\hline 33. & $\begin{array}{l}\text { Johnson D.A., Barclay R.L., Mergener K., et al. Plasma Septin9 versus fecal immunochemical testing for } \\
\text { colorectal cancer screening: a prospective multicenter study. PLoS One 2014, } 9(6) \text { :e98238. }\end{array}$ \\
\hline 34. & $\begin{array}{l}\text { Kalimutho M., Di Cecilia S., Del Vecchio Blanco G., et al. Epigenetically silenced miR-34b/c as a novel fae- } \\
\text { cal-based screening marker for colorectal cancer. Br J Cancer 2011, 104(11):1770-1778. }\end{array}$ \\
\hline 35. & $\begin{array}{l}\text { Karam R.A., Zidan H.E., Abd Elrahman T.M., et al. Study of p16 promoter methylation in Egyptian colorectal } \\
\text { cancer patients. Journal of Cellular Biochemistry } 2018 .\end{array}$ \\
\hline 36. & $\begin{array}{l}\text { Kim M.S., Louwagie J., Carvalho B., et al. Promoter DNA methylation of oncostatin m receptor-beta as a } \\
\text { novel diagnostic and therapeutic marker in colon cancer. PLoS One } 2009,4(8): e 6555 \text {. }\end{array}$ \\
\hline 37. & $\begin{array}{l}\text { Kim S.J., Tae C.H., Hong S.N., et al. EYA4 Acts as a New Tumor Suppressor Gene in Colorectal Cancer. Mol } \\
\text { Carcinog 2015, 54(12):1748-1757. }\end{array}$ \\
\hline 38. & $\begin{array}{l}\text { Kisiel J.B., Klepp P., Allawi H.T., et al. Analysis of DNA Methylation at Specific Loci in Stool Samples Detects } \\
\text { Colorectal Cancer and High-Grade Dysplasia in Patients With Inflammatory Bowel Disease. Clin Gastroen- } \\
\text { terol Hepatol } 2018 .\end{array}$ \\
\hline 39. & $\begin{array}{l}\text { Kisiel J.B., Yab T.C., Nazer Hussain F.T., et al. Stool DNA testing for the detection of colorectal neoplasia in } \\
\text { patients with inflammatory bowel disease. Aliment Pharmacol Ther 2013, 37(5):546-554. }\end{array}$ \\
\hline 40. & $\begin{array}{l}\text { Kitkumthorn N., Tuangsintanakul T., Rattanatanyong P., et al. LINE-1 methylation in the peripheral blood } \\
\text { mononuclear cells of cancer patients. Clin Chim Acta 2012, 413(9-10):869-874. }\end{array}$ \\
\hline 41. & $\begin{array}{l}\text { Kriegshauser G., Enko D., Zitt M., et al. Comparison of a prototype reverse hybridization assay and } \\
\text { MethyLight for detection of SFRP2 promotor methylation in fecal DNA. Int J Biol Markers 2017, } \\
\text { 32(4):e467-e470. }\end{array}$ \\
\hline 42. & $\begin{array}{l}\text { Leclerc D., Pham D.N., Levesque N., et al. Oncogenic role of PDK4 in human colon cancer cells. Br J Cancer } \\
\text { 2017, 116(7):930-936. }\end{array}$ \\
\hline 43. & $\begin{array}{l}\text { Lee B.B., Lee E.J., Jung E.H., et al. Aberrant methylation of APC, MGMT, RASSF2A, and Wif-1 genes in plasma } \\
\text { as a biomarker for early detection of colorectal cancer. Clin Cancer Res 2009, 15(19):6185-6191. }\end{array}$ \\
\hline
\end{tabular}




\section{CHAPTER 2}

\begin{tabular}{|c|c|}
\hline 44. & $\begin{array}{l}\text { Lenhard K., Bommer G.T., Asutay S., et al. Analysis of promoter methylation in stool: a novel method for the } \\
\text { detection of colorectal cancer. Clin Gastroenterol Hepatol 2005, 3(2):142-149. }\end{array}$ \\
\hline 45. & $\begin{array}{l}\text { Leung W.K., To K.F., Man E.P., et al. Detection of epigenetic changes in fecal DNA as a molecular screening } \\
\text { test for colorectal cancer: a feasibility study. Clin Chem 2004, 50(11):2179-2182. }\end{array}$ \\
\hline 46. & $\begin{array}{l}\text { Leung W.K., To K.F., Man E.P., et al. Quantitative detection of promoter hypermethylation in multiple genes } \\
\text { in the serum of patients with colorectal cancer. Am J Gastroenterol 2005, 100(10):2274-2279. }\end{array}$ \\
\hline 47. & $\begin{array}{l}\text { Leung W.K., To K.-F., Man E.P.S., et al. Detection of Hypermethylated DNA or Cyclooxygenase-2 Messenger } \\
\text { RNA in Fecal Samples of Patients With Colorectal Cancer or Polyps. The American Journal of Gastroenter- } \\
\text { ology 2007, 102(5):1070-1076. }\end{array}$ \\
\hline 48. & $\begin{array}{l}\text { Li W.H., Zhang H., Guo Q., et al. Detection of SNCA and FBN1 methylation in the stool as a biomarker for } \\
\text { colorectal cancer. Dis Markers 2015, 2015:657570. }\end{array}$ \\
\hline 49. & $\begin{array}{l}\text { Liu Y., Tham C.K., Ong S.Y., et al. Serum methylation levels of TAC1. SEPT9 and EYA4 as diagnostic markers } \\
\text { for early colorectal cancers: a pilot study. Biomarkers 2013, 18(5):399-405. }\end{array}$ \\
\hline 50. & $\begin{array}{l}\text { Lofton-Day C., Model F., Devos T., et al. DNA methylation biomarkers for blood-based colorectal cancer } \\
\text { screening. Clin Chem 2008, 54(2):414-423. }\end{array}$ \\
\hline 51. & $\begin{array}{l}\text { Lu H., Huang S., Zhang X., et al. DNA methylation analysis of SFRP2, GATA4/5, NDRG4 and VIM for the } \\
\text { detection of colorectal cancer in fecal DNA. Oncol Lett 2014, 8(4):1751-1756. }\end{array}$ \\
\hline 52. & $\begin{array}{l}\text { Marsit C.J., Lange C.P.E., Campan M., et al. Genome-Scale Discovery of DNA-Methylation Biomarkers for } \\
\text { Blood-Based Detection of Colorectal Cancer. PLoS ONE 2012, 7(11). }\end{array}$ \\
\hline 53. & $\begin{array}{l}\text { Mayor R., Casadome L., Azuara D., et al. Long-range epigenetic silencing at } 2 \mathrm{q} 14.2 \text { affects most human } \\
\text { colorectal cancers and may have application as a non-invasive biomarker of disease. Br J Cancer 2009, } \\
100(10): 1534-1539 .\end{array}$ \\
\hline 54. & $\begin{array}{l}\text { Melotte V., Lentjes M.H., van den Bosch S.M., et al. N-Myc downstream-regulated gene } 4 \text { (NDRG4): a } \\
\text { candidate tumor suppressor gene and potential biomarker for colorectal cancer. J Natl Cancer Inst 2009, } \\
\text { 101(13):916-927. }\end{array}$ \\
\hline 55. & $\begin{array}{l}\text { Melotte V., Yi J.M., Lentjes M.H., et al. Spectrin repeat containing nuclear envelope } 1 \text { and forkhead box } \\
\text { protein E1 are promising markers for the detection of colorectal cancer in blood. Cancer Prev Res (Phila) } \\
\text { 2015, 8(2):157-164. }\end{array}$ \\
\hline 56. & $\begin{array}{l}\text { Melson J., Li Y., Cassinotti E., et al. Commonality and differences of methylation signatures in the plasma of } \\
\text { patients with pancreatic cancer and colorectal cancer. Int J Cancer 2014,134(11):2656-2662. }\end{array}$ \\
\hline 57. & $\begin{array}{l}\text { Miotto E., Sabbioni S., Veronese A., et al. Frequent aberrant methylation of the CDH4 gene promoter in } \\
\text { human colorectal and gastric cancer. Cancer Res } 2004,64(22): 8156-8159 .\end{array}$ \\
\hline 58. & $\begin{array}{l}\text { Mitchell S.M., Ho T., Brown G.S., et al. Evaluation of Methylation Biomarkers for Detection of Circulating } \\
\text { Tumor DNA and Application to Colorectal Cancer. Genes (Basel) 2016, 7(12). }\end{array}$ \\
\hline 59. & $\begin{array}{l}\text { Müller H.M., Oberwalder M., Fiegl H., et al. Methylation changes in faecal DNA: a marker for colorectal } \\
\text { cancer screening? The Lancet 2004, 363(9417):1283-1285. }\end{array}$ \\
\hline 60. & $\begin{array}{l}\text { Nagai Y., Sunami E., Yamamoto Y., et al. LINE-1 hypomethylation status of circulating cell-free DNA in } \\
\text { plasma as a biomarker for colorectal cancer. Oncotarget 2017, 8(7):11906-11916. }\end{array}$ \\
\hline 61. & $\begin{array}{l}\text { Nagasaka T., Tanaka N., Cullings H.M., et al. Analysis of fecal DNA methylation to detect gastrointestinal } \\
\text { neoplasia. J Natl Cancer Inst 2009, 101(18):1244-1258. }\end{array}$ \\
\hline 62. & $\begin{array}{l}\text { Najbauer J., Grützmann R., Molnar B., et al. Sensitive Detection of Colorectal Cancer in Peripheral Blood by } \\
\text { Septin } 9 \text { DNA Methylation Assay. PLoS ONE 2008, 3(11). }\end{array}$ \\
\hline 63. & $\begin{array}{l}\text { Nan H., Giovannucci E.L., Wu K., et al. Pre-diagnostic leukocyte genomic DNA methylation and the risk of } \\
\text { colorectal cancer in women. PLoS One 2013, 8(4):e59455. }\end{array}$ \\
\hline 64. & $\begin{array}{l}\text { Nishio M., Sakakura C., Nagata T., et al. RUNX3 promoter methylation in colorectal cancer: its relationship } \\
\text { with microsatellite instability and its suitability as a novel serum tumor marker. Anticancer Res } 2010 \text {, } \\
30(7): 2673-2682 \text {. }\end{array}$ \\
\hline
\end{tabular}




\begin{tabular}{|c|c|}
\hline 65. & $\begin{array}{l}\text { Niu F., Wen J., Fu X., et al. Stool DNA Test of Methylated Syndecan-2 for the Early Detection of Colorectal } \\
\text { Neoplasia. Cancer Epidemiology Biomarkers \& Prevention 2017, 26(9):1411-1419. }\end{array}$ \\
\hline 66. & $\begin{array}{l}\text { Nunes S.P., Moreira-Barbosa C., Salta S., et al. Cell-free DNA methylation of selected genes allows for early } \\
\text { detection of the major cancers in women. Cancers } 2018,10(10): 357 .\end{array}$ \\
\hline 67. & $\begin{array}{l}\text { Oh T., Kim N., Moon Y., et al. Genome-wide identification and validation of a novel methylation biomarker, } \\
\text { SDC2, for blood-based detection of colorectal cancer. J Mol Diagn 2013, 15(4):498-507. }\end{array}$ \\
\hline 68. & $\begin{array}{l}\text { Oh T.J., Oh H.I., Seo Y.Y., et al. Feasibility of quantifying SDC2 methylation in stool DNA for early detection of } \\
\text { colorectal cancer. Clin Epigenetics 2017, 9:126. }\end{array}$ \\
\hline 69. & $\begin{array}{l}\text { Orntoft M.B., Nielsen H.J., Orntoft T.F., et al. Performance of the colorectal cancer screening marker Sept9 is } \\
\text { influenced by age, diabetes and arthritis: a nested case-control study. BMC Cancer 2015, 15:819. }\end{array}$ \\
\hline 70 . & $\begin{array}{l}\text { Pack S.C., Kim H.R., Lim S.W., et al. Usefulness of plasma epigenetic changes of five major genes involved in } \\
\text { the pathogenesis of colorectal cancer. Int J Colorectal Dis 2013, 28(1):139-147. }\end{array}$ \\
\hline 71. & $\begin{array}{l}\text { Park S.K., Baek H.L., Yu J., et al. Is methylation analysis of SFRP2, TFPI2, NDRG4, and BMP3 promoters } \\
\text { suitable for colorectal cancer screening in the Korean population? Intest Res 2017, 15(4):495-501. }\end{array}$ \\
\hline 72. & $\begin{array}{l}\text { Pedersen S.K., Baker R.T., McEvoy A., et al. A two-gene blood test for methylated DNA sensitive for colorec- } \\
\text { tal cancer. PLoS One 2015, 10(4):e0125041. }\end{array}$ \\
\hline 73. & $\begin{array}{l}\text { Pedersen S.K., Mitchell S.M., Graham L.D., et al. CAHM, a long non-coding RNA gene hypermethylated in } \\
\text { colorectal neoplasia. Epigenetics 2014, 9(8):1071-1082. }\end{array}$ \\
\hline 74. & $\begin{array}{l}\text { Pedersen S.K., Symonds E.L., Baker R.T., et al. Evaluation of an assay for methylated BCAT1 and IKZF1 in } \\
\text { plasma for detection of colorectal neoplasia. BMC Cancer 2015, 15:654. }\end{array}$ \\
\hline 75. & $\begin{array}{l}\text { Potter N.T., Hurban P., White M.N., et al. Validation of a real-time PCR-based qualitative assay for the detec- } \\
\text { tion of methylated SEPT9 DNA in human plasma. Clin Chem 2014, 60(9):1183-1191. }\end{array}$ \\
\hline 76. & $\begin{array}{l}\text { Rasmussen S.L., Krarup H.B., Sunesen K.G., et al. Hypermethylated DNA, a circulating biomarker for } \\
\text { colorectal cancer detection. PLoS One 2017, 12(7):e0180809. }\end{array}$ \\
\hline 77. & $\begin{array}{l}\text { Ravegnini G., Zolezzi Moraga J.M., Maffei F., et al. Simultaneous Analysis of SEPT9 Promoter Methylation } \\
\text { Status, Micronuclei Frequency, and Folate-Related Gene Polymorphisms: The Potential for a Novel Blood- } \\
\text { Based Colorectal Cancer Biomarker. Int J Mol Sci 2015, 16(12):28486-28497. }\end{array}$ \\
\hline 78. & $\begin{array}{l}\text { Rezvani N., Alibakhshi R., Vaisi-Raygani A., et al. Detection of SPG20 gene promoter-methylated DNA, as a } \\
\text { novel epigenetic biomarker, in plasma for colorectal cancer diagnosis using the MethyLight method. Oncol } \\
\text { Lett } 2017,13(5): 3277-3284 \text {. }\end{array}$ \\
\hline 79. & $\begin{array}{l}\text { Roperch J.P., Incitti R., Forbin S., et al. Aberrant methylation of NPY, PENK, and WIF1 as a promising mark- } \\
\text { er for blood-based diagnosis of colorectal cancer. BMC Cancer 2013, 13:566. }\end{array}$ \\
\hline 80. & $\begin{array}{l}\text { Sabbioni S., Miotto E., Veronese A., et al. Multigene methylation analysis of gastrointestinal tumors: TPEF } \\
\text { emerges as a frequent tumor-specific aberrantly methylated marker that can be detected in peripheral } \\
\text { blood. Mol Diagn } 2003,7(3-4): 201-207 \text {. }\end{array}$ \\
\hline 81. & $\begin{array}{l}\text { Sakamoto J., Fujiya M., Okamoto K., et al. Immunoprecipitation of nucleosomal DNA is a novel procedure } \\
\text { to improve the sensitivity of serum screening for the p16 hypermethylation associated with colon cancer. } \\
\text { Cancer Epidemiol 2010, 34(2):194-199. }\end{array}$ \\
\hline 82. & $\begin{array}{l}\text { Salehi R., Atapour N., Vatandoust N., et al. Methylation pattern of ALX4 gene promoter as a potential bio- } \\
\text { marker for blood-based early detection of colorectal cancer. Adv Biomed Res 2015, 4:252. }\end{array}$ \\
\hline 83. & $\begin{array}{l}\text { Salehi R., Mohammadi M., Emami M.H., et al. Methylation pattern of SFRP1 promoter in stool sample is a } \\
\text { potential marker for early detection of colorectal cancer. Adv Biomed Res 2012, 1:87. }\end{array}$ \\
\hline 84. & $\begin{array}{l}\text { Shirahata A., Hibi K. Serum vimentin methylation as a potential marker for colorectal cancer. Anticancer } \\
\text { Res 2014, 34(8):4121-4125. }\end{array}$ \\
\hline 85. & $\begin{array}{l}\text { Song B.P., Jain S., Lin S.Y., et al. Detection of hypermethylated vimentin in urine of patients with colorectal } \\
\text { cancer. J Mol Diagn 2012, 14(2):112-119. }\end{array}$ \\
\hline
\end{tabular}




\section{CHAPTER 2}

\begin{tabular}{|c|c|}
\hline 86. & $\begin{array}{l}\text { Song L., Wang J., Wang H., et al. The quantitative profiling of blood mSEPT9 determines the detection } \\
\text { performance on colorectal tumors. Epigenomics } 2018,10(12): 1569-1583 \text {. }\end{array}$ \\
\hline 87. & $\begin{array}{l}\text { Suehiro Y., Hashimoto S., Higaki S., et al. Blood free-circulating DNA testing by highly sensitive methylation } \\
\text { assay to diagnose colorectal neoplasias. Oncotarget 2018, 9(24):16974-16987. }\end{array}$ \\
\hline 88. & $\begin{array}{l}\text { Suehiro Y., Zhang Y., Hashimoto S., et al. Highly sensitive faecal DNA testing of TWIST1 methylation in } \\
\text { combination with faecal immunochemical test for haemoglobin is a promising marker for detection of } \\
\text { colorectal neoplasia. Annals of Clinical Biochemistry 2018, 55(1):59-68. }\end{array}$ \\
\hline 89. & $\begin{array}{l}\text { Symonds E.L., Pedersen S.K., Baker R.T., et al. A Blood Test for Methylated BCAT1 and IKZF1 vs. a Fecal } \\
\text { Immunochemical Test for Detection of Colorectal Neoplasia. Clin Transl Gastroenterol 2016, 7:e137. }\end{array}$ \\
\hline 90. & $\begin{array}{l}\text { Takane K., Midorikawa Y., Yagi K., et al. Aberrant promoter methylation of PPP1R3C and EFHD1 in plasma } \\
\text { of colorectal cancer patients. Cancer Med 2014, 3(5):1235-1245. }\end{array}$ \\
\hline 91. & $\begin{array}{l}\text { Tang D., Liu J., Wang D.R., et al. Diagnostic and prognostic value of the methylation status of secreted } \\
\text { frizzled-related protein } 2 \text { in colorectal cancer. Clin Invest Med 2011, 34(2):E88-95. }\end{array}$ \\
\hline 92. & $\begin{array}{l}\text { Tanzer M., Balluff B., Distler J., et al. Performance of epigenetic markers SEPT9 and ALX4 in plasma for } \\
\text { detection of colorectal precancerous lesions. PLoS One 2010,5(2):e9061. }\end{array}$ \\
\hline 93. & $\begin{array}{l}\text { Toth K., Sipos F., Kalmar A., et al. Detection of methylated SEPT9 in plasma is a reliable screening method } \\
\text { for both left- and right-sided colon cancers. PLoS One 2012, 7(9):e46000. }\end{array}$ \\
\hline 94. & $\begin{array}{l}\text { Toth K., Wasserkort R., Sipos F., et al. Detection of methylated septin } 9 \text { in tissue and plasma of colorectal } \\
\text { patients with neoplasia and the relationship to the amount of circulating cell-free DNA. PLoS One 2014, } \\
9(12): \mathrm{e} 115415 .\end{array}$ \\
\hline 95. & $\begin{array}{l}\text { Wang D.R., Tang D. Hypermethylated SFRP2 gene in fecal DNA is a high potential biomarker for colorectal } \\
\text { cancer noninvasive screening. World J Gastroenterol } 2008,14(4): 524-531 .\end{array}$ \\
\hline 96. & $\begin{array}{l}\text { Wang Y.C., Yu Z.H., Liu C., et al. Detection of RASSF1A promoter hypermethylation in serum from gastric } \\
\text { and colorectal adenocarcinoma patients. World J Gastroenterol 2008, 14(19):3074-3080. }\end{array}$ \\
\hline 97. & $\begin{array}{l}\text { Warren J.D., Xiong W., Bunker A.M., et al. Septin } 9 \text { methylated DNA is a sensitive and specific blood test for } \\
\text { colorectal cancer. BMC Med 2011, 9:133. }\end{array}$ \\
\hline 98. & $\begin{array}{l}\text { Wu D., Zhou G., Jin P., et al. Detection of Colorectal Cancer Using a Simplified SEPT9 Gene Methylation } \\
\text { Assay Is a Reliable Method for Opportunistic Screening. J Mol Diagn 2016, 18(4):535-545. }\end{array}$ \\
\hline 99. & $\begin{array}{l}\text { Wu P.P., Zou J.H., Tang R.N., et al. Detection and Clinical Significance of DLC1 Gene Methylation in Serum } \\
\text { DNA from Colorectal Cancer Patients. Chin J Cancer Res 2011, 23(4):283-287. }\end{array}$ \\
\hline 100. & $\begin{array}{l}\text { Wu X.D., Song Y.C., Cao P.L., et al. Detection of miR-34a and miR-34b/c in stool sample as potential screen- } \\
\text { ing biomarkers for noninvasive diagnosis of colorectal cancer. Med Oncol 2014, 31(4):894. }\end{array}$ \\
\hline 101. & $\begin{array}{l}\text { Xiao W., Zhao H., Dong W., et al. Quantitative detection of methylated NDRG4 gene as a candidate biomark- } \\
\text { er for diagnosis of colorectal cancer. Oncol Lett 2015, 9(3):1383-1387. }\end{array}$ \\
\hline 102. & $\begin{array}{l}\text { Xiao Z., Li B., Wang G., et al. Validation of methylation-sensitive high-resolution melting (MS-HRM) for the } \\
\text { detection of stool DNA methylation in colorectal neoplasms. Clin Chim Acta 2014, 431:154-163. }\end{array}$ \\
\hline 103. & $\begin{array}{l}\text { Xie L., Jiang X., Li Q., et al. Diagnostic value of methylated Septin9 for colorectal cancer detection. Frontiers } \\
\text { in Oncology 2018, 8(JUL):247. }\end{array}$ \\
\hline 104. & $\begin{array}{l}\text { Zhang H., Song Y.C., Dang C.X. Detection of hypermethylated spastic paraplegia-20 in stool samples of } \\
\text { patients with colorectal cancer. Int J Med Sci } 2013,10(3): 230-234 \text {. }\end{array}$ \\
\hline 105. & $\begin{array}{l}\text { Zhang J., Yang S., Xie Y., et al. Detection of methylated tissue factor pathway inhibitor } 2 \text { and human long } \\
\text { DNA in fecal samples of patients with colorectal cancer in China. Cancer Epidemiol 2012, 36(1):73-77. }\end{array}$ \\
\hline 106. & $\begin{array}{l}\text { Zhang T., Cui G., Yao Y.L., et al. Value of CNRIP1 promoter methylation in colorectal cancer screening and } \\
\text { prognosis assessment and its influence on the activity of cancer cells. Arch Med Sci 2017, 13(6):1281- } \\
1294 .\end{array}$ \\
\hline 107. & $\begin{array}{l}\text { Zhang W., Bauer M., Croner R.S., et al. DNA Stool Test for Colorectal Cancer: Hypermethylation of the } \\
\text { Secreted Frizzled-Related Protein-1 Gene. Diseases of the Colon \& Rectum 2007, 50(10):1618-1627. }\end{array}$ \\
\hline
\end{tabular}


$108 . \quad$ Zhang X., Song Y.F., Lu H.N., et al. Combined detection of plasma GATA5 and SFRP2 methylation is a valid noninvasive biomarker for colorectal cancer and adenomas. World J Gastroenterol 2015, 21(9):26292637.

109. Zhou D., Tang W., Su G., et al. PCDH18 is frequently inactivated by promoter methylation in colorectal cancer. Sci Rep 2017, 7(1):2819.

Supplementary Table 4. Overview of the study characteristics of 109 included studies. 


\begin{tabular}{|c|c|c|c|c|c|c|c|c|c|}
\hline 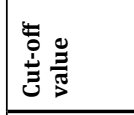 & & 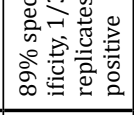 & & & & & & & \\
\hline 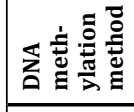 & $\frac{\bar{n}}{\Sigma}$ & 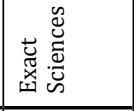 & 苴 & 氡 & $\frac{\tilde{n}}{\Sigma}$ & $\sum_{\Sigma}^{\tilde{n}}$ & & & \\
\hline 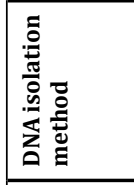 & 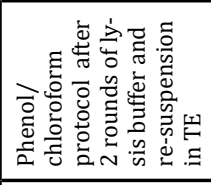 & 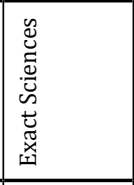 & 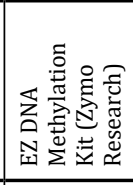 & 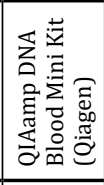 & 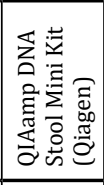 & 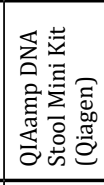 & & & \\
\hline$\stackrel{\check{\varpi}}{\beth}$ & & & 官 & 高蒿 & & 今ે̀ & & & \\
\hline 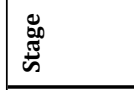 & $\begin{array}{l}u \\
\omega\end{array}$ & 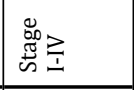 & 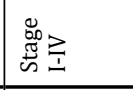 & & & 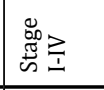 & & & \\
\hline 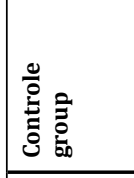 & & 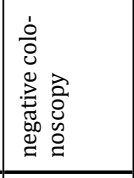 & 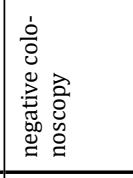 & 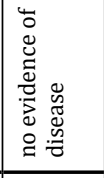 & 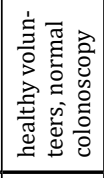 & 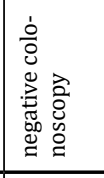 & & & \\
\hline 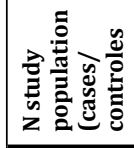 & $\underset{\stackrel{N}{N}}{\stackrel{N}{N}}$ & 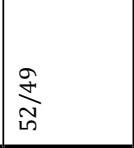 & 站 & \begin{tabular}{|l}
$\hat{m}$ \\
$\hat{\sigma}$ \\
in
\end{tabular} & 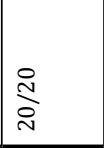 & 命 & & & \\
\hline 高 & 苂 & 芯 & 苂 & $\ddot{\mathscr{U}}$ & $\ddot{\mathscr{U}}$ & $\ddot{\mathscr{\Xi}}$ & & & \\
\hline 造 & \begin{tabular}{|l}
$\overrightarrow{0}$ \\
$\dot{D}$ \\
\end{tabular} & 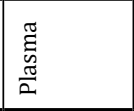 & 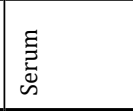 & \begin{tabular}{|l|}
$\frac{\pi}{5}$ \\
$\frac{\pi}{\tilde{E}}$ \\
$\frac{\pi}{2}$ \\
\end{tabular} & 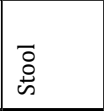 & $\begin{array}{l}\overrightarrow{0} \\
\dot{D} \\
\dot{n}\end{array}$ & & & \\
\hline 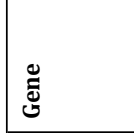 & 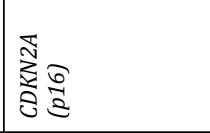 & 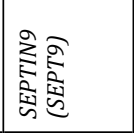 & 価 & 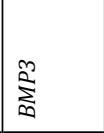 & 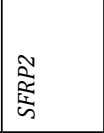 & 芳 & 龍 & 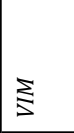 & 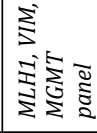 \\
\hline 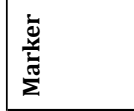 & 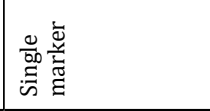 & 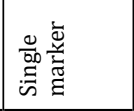 & 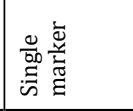 & 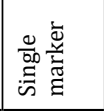 & 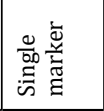 & 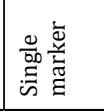 & 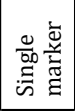 & 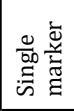 & 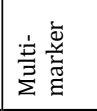 \\
\hline 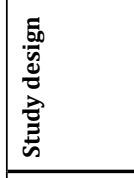 & 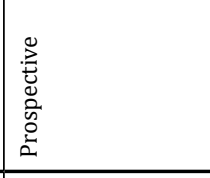 & 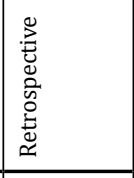 & 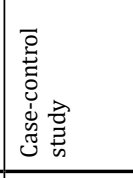 & 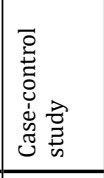 & 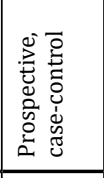 & 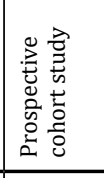 & & & \\
\hline 宸 & 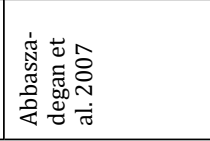 & 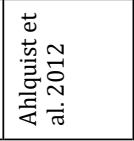 & 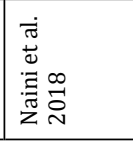 & 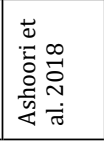 & 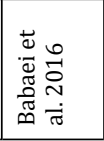 & 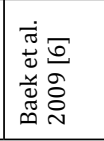 & & & \\
\hline
\end{tabular}


DIAGNOSTIC DNA METHYLATION BIOMARKERS

\begin{tabular}{|c|c|c|c|c|c|c|c|c|c|c|c|c|c|}
\hline 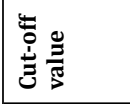 & & & & & & $\begin{array}{l}{ }_{0} \\
\sum_{\Sigma}^{\hat{L}}\end{array}$ & $\begin{array}{l}\stackrel{0}{\hat{n}} \\
\sum_{\Sigma} \\
\end{array}$ & $\mid \begin{array}{l}0 \\
\sum_{\underline{\Delta}} \\
\underline{\Sigma}\end{array}$ & & & & & \\
\hline 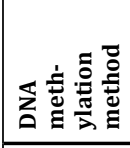 & 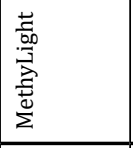 & & & & $\begin{array}{l}\frac{n}{n} \\
\Sigma\end{array}$ & $\begin{array}{l}\tilde{\Xi} \\
\ddot{\sigma}\end{array}$ & & & 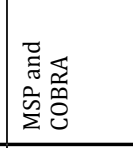 & & & & \\
\hline 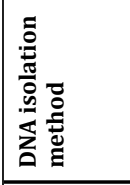 & 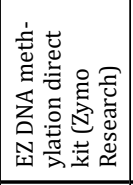 & & & & 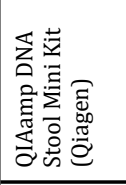 & 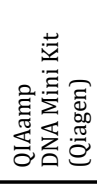 & & & 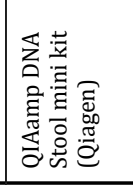 & & & & \\
\hline 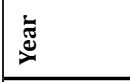 & & & & & & & & & & & & & \\
\hline 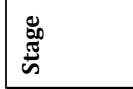 & 穻完 & & & & & $\begin{array}{l}\text { 总 } \\
\text { 总 } \\
\text { W. }\end{array}$ & & & & & & & \\
\hline 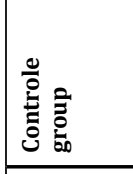 & & & & & 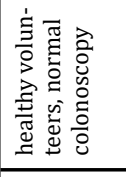 & 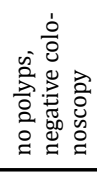 & & & 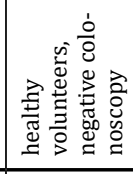 & & & & \\
\hline 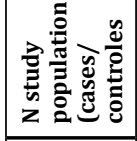 & 醇 & & & & ì & 孞 & & & $\underset{\Im}{\stackrel{I}{I}}$ & & & & \\
\hline 亩 & 岇 & & & & 岇 & 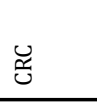 & & & Æֶّ & & & & \\
\hline 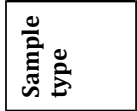 & \begin{tabular}{|l|}
$\frac{\pi}{5}$ \\
$\frac{\sigma}{2}$ \\
$\frac{\pi}{2}$
\end{tabular} & & & & \begin{tabular}{|l}
$\vec{o}$ \\
$\dot{d}$ \\
$i s$
\end{tabular} & 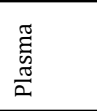 & & & $\begin{array}{l}\overrightarrow{0} \\
\dot{D} \\
\dot{\omega}\end{array}$ & & & & \\
\hline E⿱ & 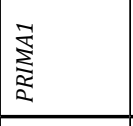 & $\begin{array}{l}\tilde{U} \\
\omega \\
\omega\end{array}$ & 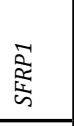 & 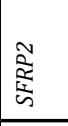 & 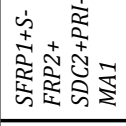 & 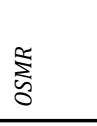 & 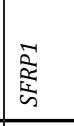 & 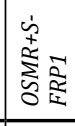 & 岑 & 章 & 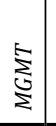 & $\begin{array}{l}\mathbf{z} \\
\Sigma\end{array}$ & 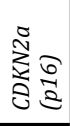 \\
\hline 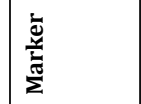 & 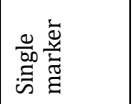 & 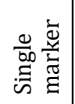 & 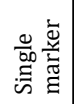 & 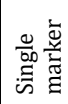 & 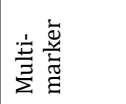 & 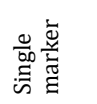 & 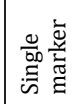 & 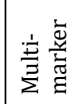 & 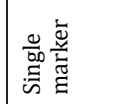 & 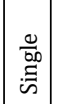 & 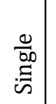 & 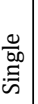 & 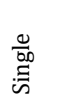 \\
\hline 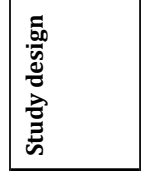 & 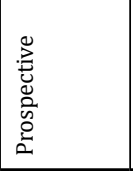 & & & & & 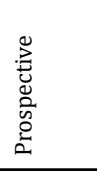 & & & 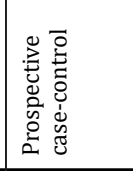 & & & & \\
\hline 䓫 & 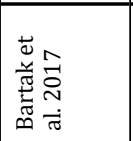 & & & & & 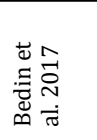 & & & 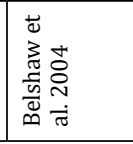 & & & & \\
\hline
\end{tabular}




\begin{tabular}{|c|c|c|c|c|c|c|c|c|c|c|c|}
\hline & & & $\begin{array}{l}\text { Ln } \\
\text { - }\end{array}$ & $\stackrel{0}{\dot{m}^{\prime}}$ & 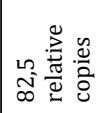 & & $\begin{array}{l}\text { Ln } \\
\text { - }\end{array}$ & $\overrightarrow{0}$ & 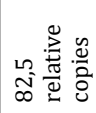 & & 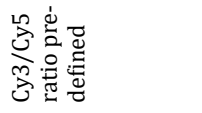 \\
\hline & & & $\sum_{\sigma}^{\infty}$ & & & & $\sum_{\text {¿n }}^{n}$ & & & & 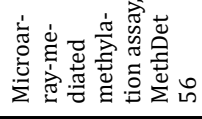 \\
\hline & & & 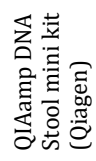 & & & & 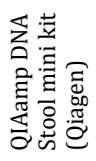 & & & & 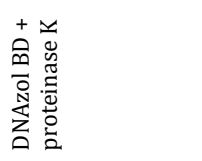 \\
\hline & & & 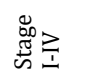 & & & & 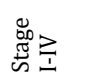 & & & & 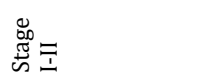 \\
\hline & & & 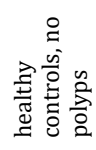 & & & & 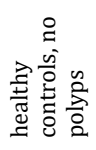 & & & & 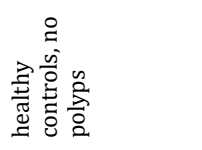 \\
\hline & & & 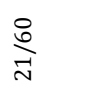 & & 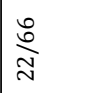 & & $\stackrel{\stackrel{m}{f}}{\dot{f}}$ & & & & 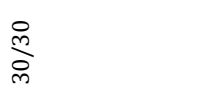 \\
\hline & & & U. & & & & تِّ & & & & ن̛ \\
\hline & & & $\begin{array}{l}\overline{0} \\
\text { 号 } \\
\text { in }\end{array}$ & & & & $\begin{array}{l}\overrightarrow{8} \\
\text { is }\end{array}$ & & & & $\begin{array}{l}\frac{\pi}{\tilde{H}} \\
\frac{\pi}{2} \\
\frac{\pi}{2}\end{array}$ \\
\hline 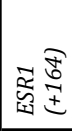 & 趉 & 疍离 & 胥 & క్ర & 啇 & 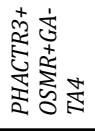 & 离 & క్ & 啇 & 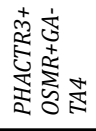 & 胥 \\
\hline 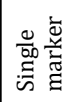 & 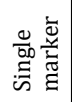 & 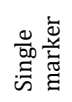 & 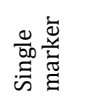 & 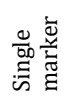 & 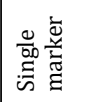 & 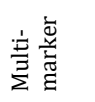 & 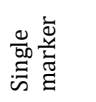 & 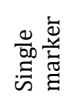 & 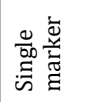 & 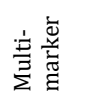 & 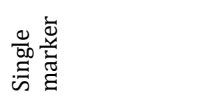 \\
\hline & & & & & & & & & & & 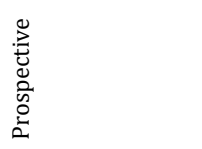 \\
\hline & & & 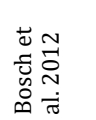 & & & & & & & & 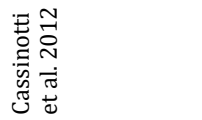 \\
\hline
\end{tabular}


DIAGNOSTIC DNA METHYLATION BIOMARKERS

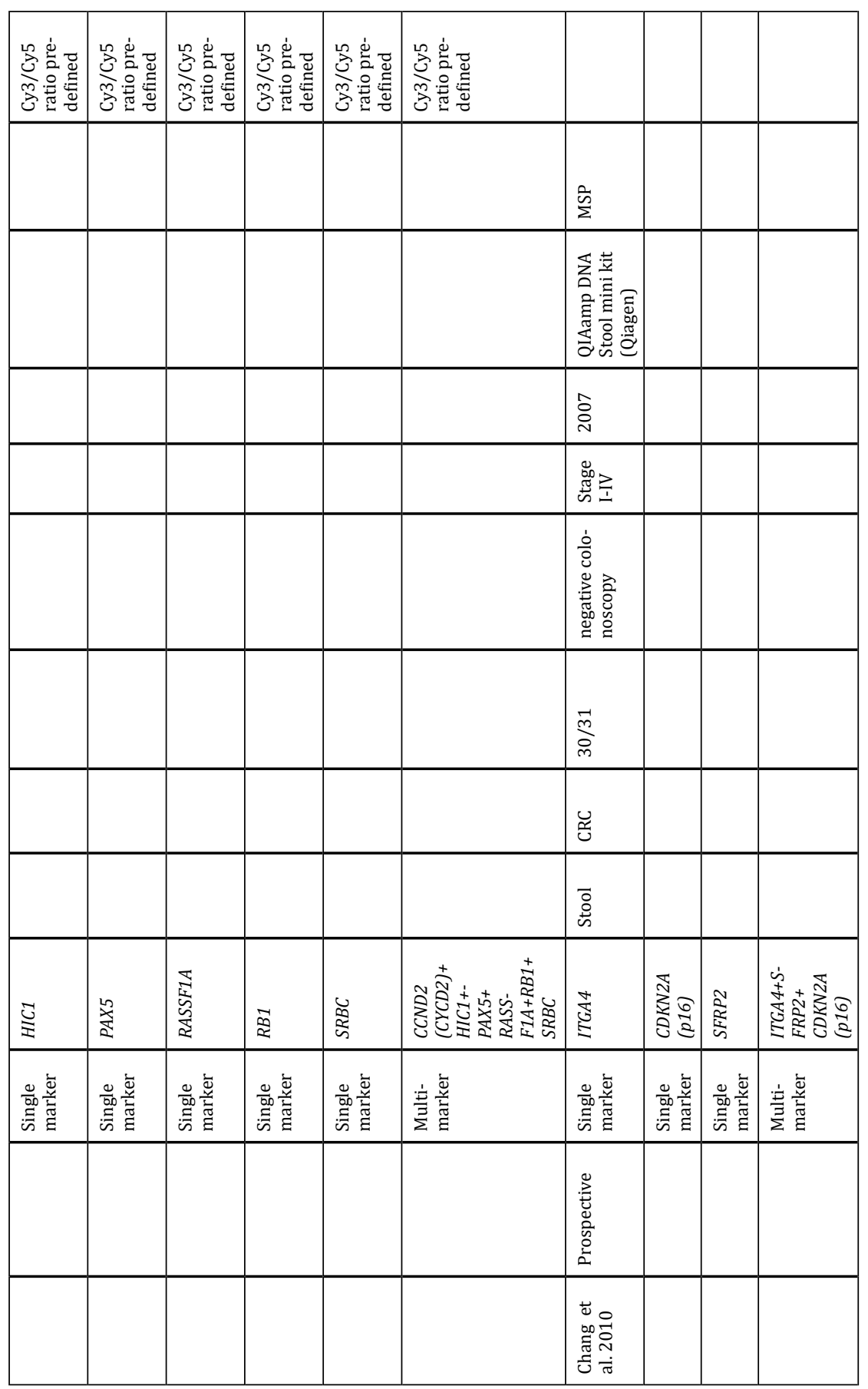


CHAPTER 2

\begin{tabular}{|c|c|c|c|c|c|c|c|c|}
\hline & & 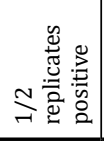 & 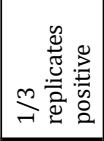 & me & 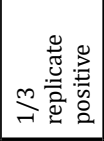 & 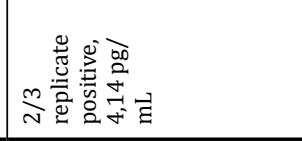 & 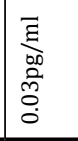 & \\
\hline 总 & 竞 & 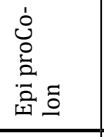 & & 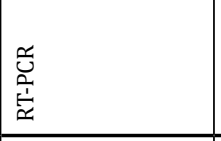 & & 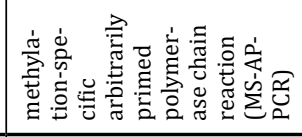 & & 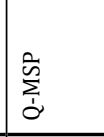 \\
\hline 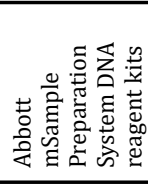 & & 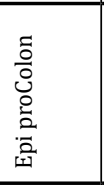 & & 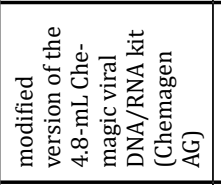 & & 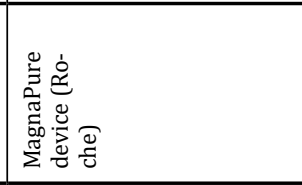 & & 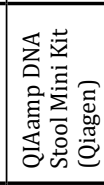 \\
\hline ثิ่ & & 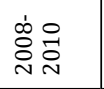 & & & & & & \\
\hline 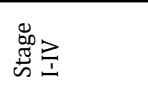 & 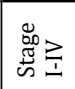 & 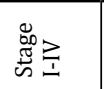 & & 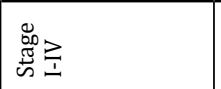 & 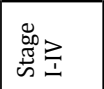 & & & \\
\hline 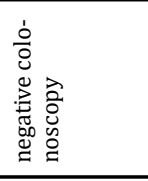 & 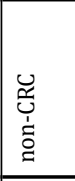 & 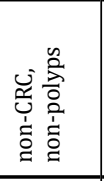 & & & & & & 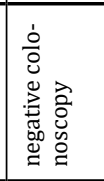 \\
\hline 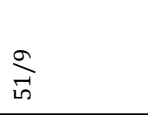 & $\stackrel{\infty}{\stackrel{\infty}{\xi}}$ & 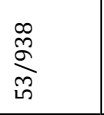 & iे & 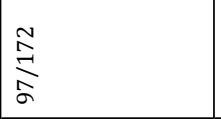 & 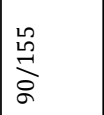 & 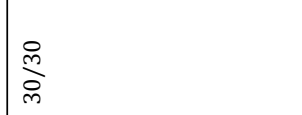 & & 帝 \\
\hline ्ֶञ & Ü & : & & ت્ & : & 艺 & : & 苂 \\
\hline$\frac{\vec{\circ}}{\omega}$ & 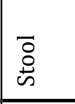 & 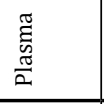 & & 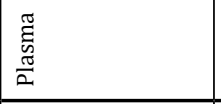 & & \begin{tabular}{|l} 
竞 \\
品
\end{tabular} & 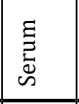 & \begin{tabular}{|l} 
\\
\\
\\
\end{tabular} \\
\hline 之⿳亠口冋. & $\S$ & 高 & 骇高 & 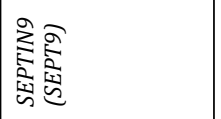 & 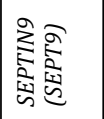 & 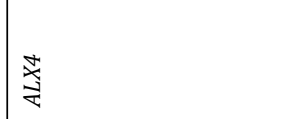 & 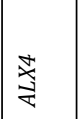 & $\ddot{z}$ \\
\hline 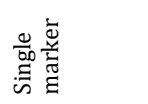 & 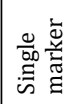 & 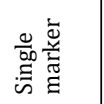 & 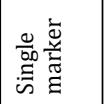 & 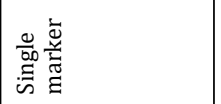 & 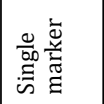 & 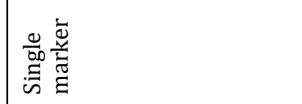 & 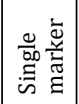 & 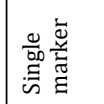 \\
\hline 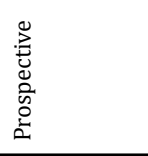 & 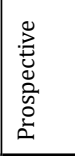 & 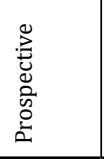 & & 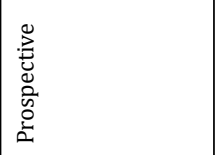 & & & & 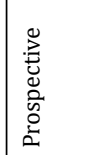 \\
\hline 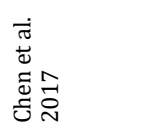 & 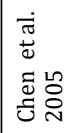 & 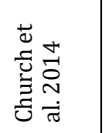 & & 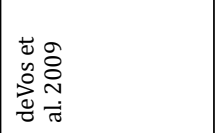 & & 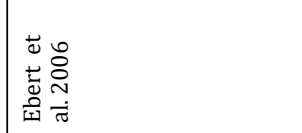 & & 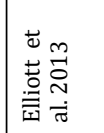 \\
\hline
\end{tabular}


DIAGNOSTIC DNA METHYLATION BIOMARKERS

\begin{tabular}{|c|c|c|c|c|c|c|c|c|c|c|}
\hline & & & & & 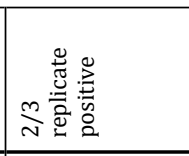 & 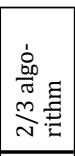 & & & & \\
\hline & & & & & 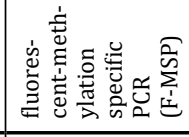 & 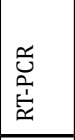 & 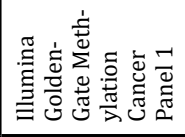 & $\sum_{i}^{\infty}$ & & \\
\hline & & & & & 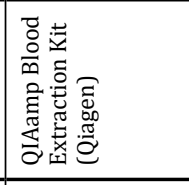 & 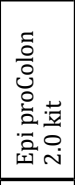 & & 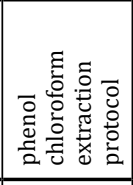 & 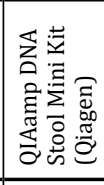 & 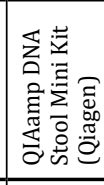 \\
\hline & & & & & & 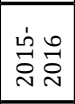 & 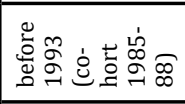 & & & \\
\hline & & & & & & 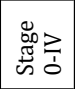 & & 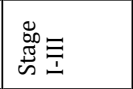 & 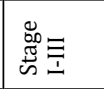 & 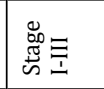 \\
\hline & & & & & & 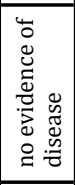 & & 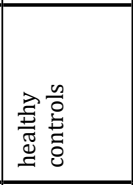 & 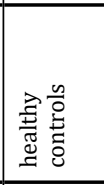 & 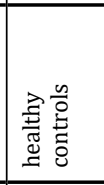 \\
\hline & & & & & $\underset{\sim}{\dot{\infty}}$ & 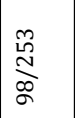 & 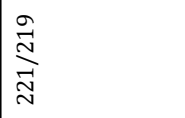 & $\underset{\exists}{\stackrel{7}{ت}}$ & 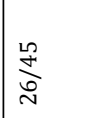 & 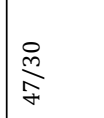 \\
\hline & & & & & 苂 & $\ddot{\mathscr{U}}$ & Ü & ֶ̈ & 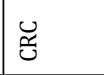 & 苂 \\
\hline & & & & & \begin{tabular}{|l}
$\frac{\pi}{5}$ \\
$\frac{\pi}{\tilde{m}}$ \\
$\frac{\pi}{2}$
\end{tabular} & \begin{tabular}{|l}
$\frac{\pi}{5}$ \\
$\frac{\pi}{\tilde{m}}$ \\
$\frac{\pi}{2}$
\end{tabular} & 递 & $\begin{array}{l}\overline{\mathrm{o}} \\
\mathrm{s}\end{array}$ & $\begin{array}{l}\overline{0} \\
\dot{D} \\
\dot{n}\end{array}$ & $\begin{array}{l}\overline{8} \\
\dot{8} \\
\dot{w}\end{array}$ \\
\hline 弪 & 密 & 촐 & 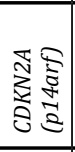 & 然 & 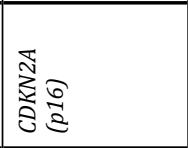 & 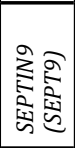 & 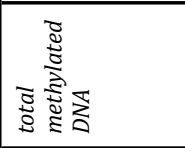 & 愛 & 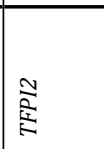 & $\mid \frac{N}{2}$ \\
\hline 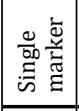 & 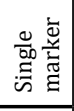 & 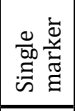 & 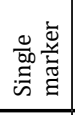 & 蒿离 & 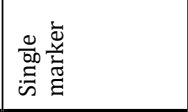 & 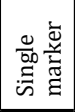 & & 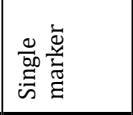 & 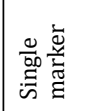 & 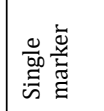 \\
\hline & & & & & & & 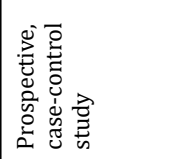 & 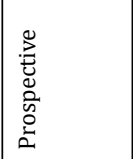 & & \\
\hline & & & & & 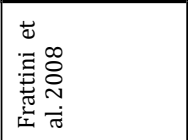 & 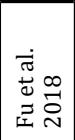 & 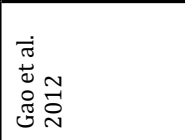 & 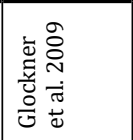 & & \\
\hline
\end{tabular}




\begin{tabular}{|c|c|c|c|c|c|c|c|c|c|c|c|c|}
\hline & & 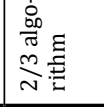 & $\vec{\infty}$ & $\vec{\infty}$ & & & & & $\begin{array}{l}0 \\
0 \\
0 \\
0 \\
1 \\
\Lambda\end{array}$ & & & \\
\hline$\sum_{\bar{n}}^{n}$ & 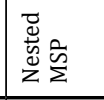 & 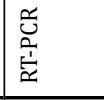 & 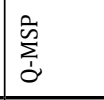 & & 站 & & & & 离 & $\frac{\tilde{n}}{\Sigma}$ & $\frac{\tilde{n}}{\Sigma}$ & \\
\hline 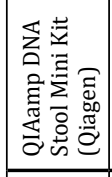 & 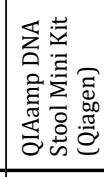 & 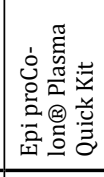 & 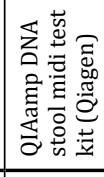 & & 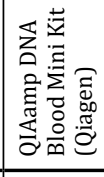 & & & & 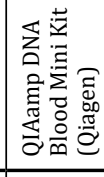 & 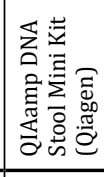 & 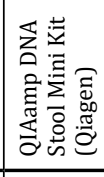 & \\
\hline ثิ่ & & & & & & & & & & & & \\
\hline 品 & & 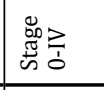 & 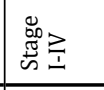 & 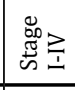 & & & & & 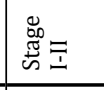 & & & \\
\hline & 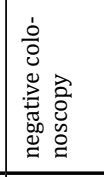 & 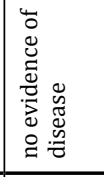 & & & & & & & 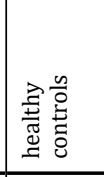 & 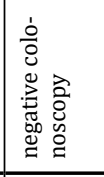 & 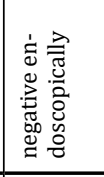 & \\
\hline 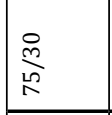 & 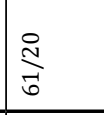 & 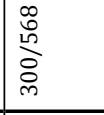 & 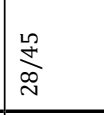 & 站 & 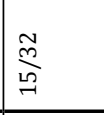 & 告 & 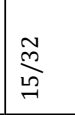 & 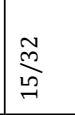 & 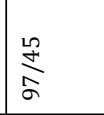 & 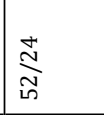 & $\mid \begin{array}{c}\text { N } \\
\end{array}$ & \\
\hline ্ֶّ & 苾 & 苂 & 艺 & & 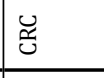 & & & & 苂 & 苂 & 苂 & \\
\hline 宫 & $\begin{array}{l}\overline{8} \\
\dot{8} \\
\end{array}$ & 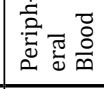 & $\overline{8}$ & & 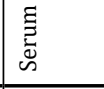 & & & & & \begin{tabular}{|l}
$\overrightarrow{0}$ \\
$\dot{8}$ \\
in
\end{tabular} & \begin{tabular}{|l}
$\overline{0}$ \\
$\dot{0}$ \\
is
\end{tabular} & \\
\hline 总 & 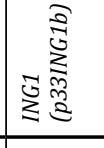 & 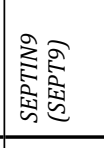 & \begin{tabular}{|l} 
\\
Uू \\
Uू \\
\end{tabular} & \begin{tabular}{|l} 
\\
竎 \\
竎 \\
\end{tabular} & 莣 & 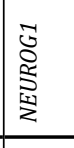 & 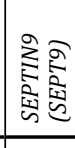 & ミ & 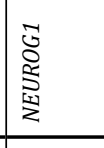 & 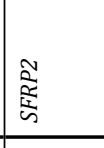 & \begin{tabular}{|l}
$\vdots$ \\
$\vdots$ \\
\end{tabular} & \begin{tabular}{|l}
5 \\
s. \\
2 \\
\end{tabular} \\
\hline 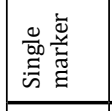 & 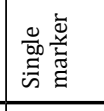 & 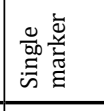 & 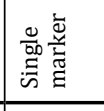 & 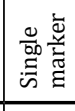 & 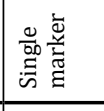 & 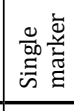 & 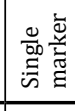 & 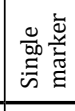 & 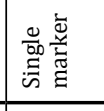 & 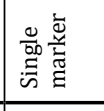 & 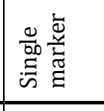 & 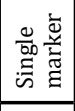 \\
\hline 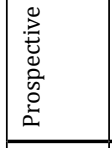 & & 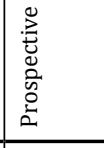 & 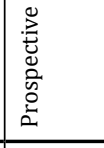 & & 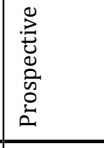 & & & & & 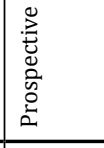 & 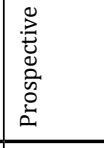 & \\
\hline 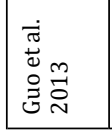 & 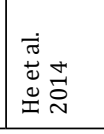 & 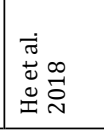 & 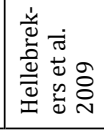 & & 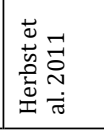 & & & & & 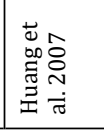 & 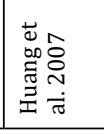 & \\
\hline
\end{tabular}


DIAGNOSTIC DNA METHYLATION BIOMARKERS

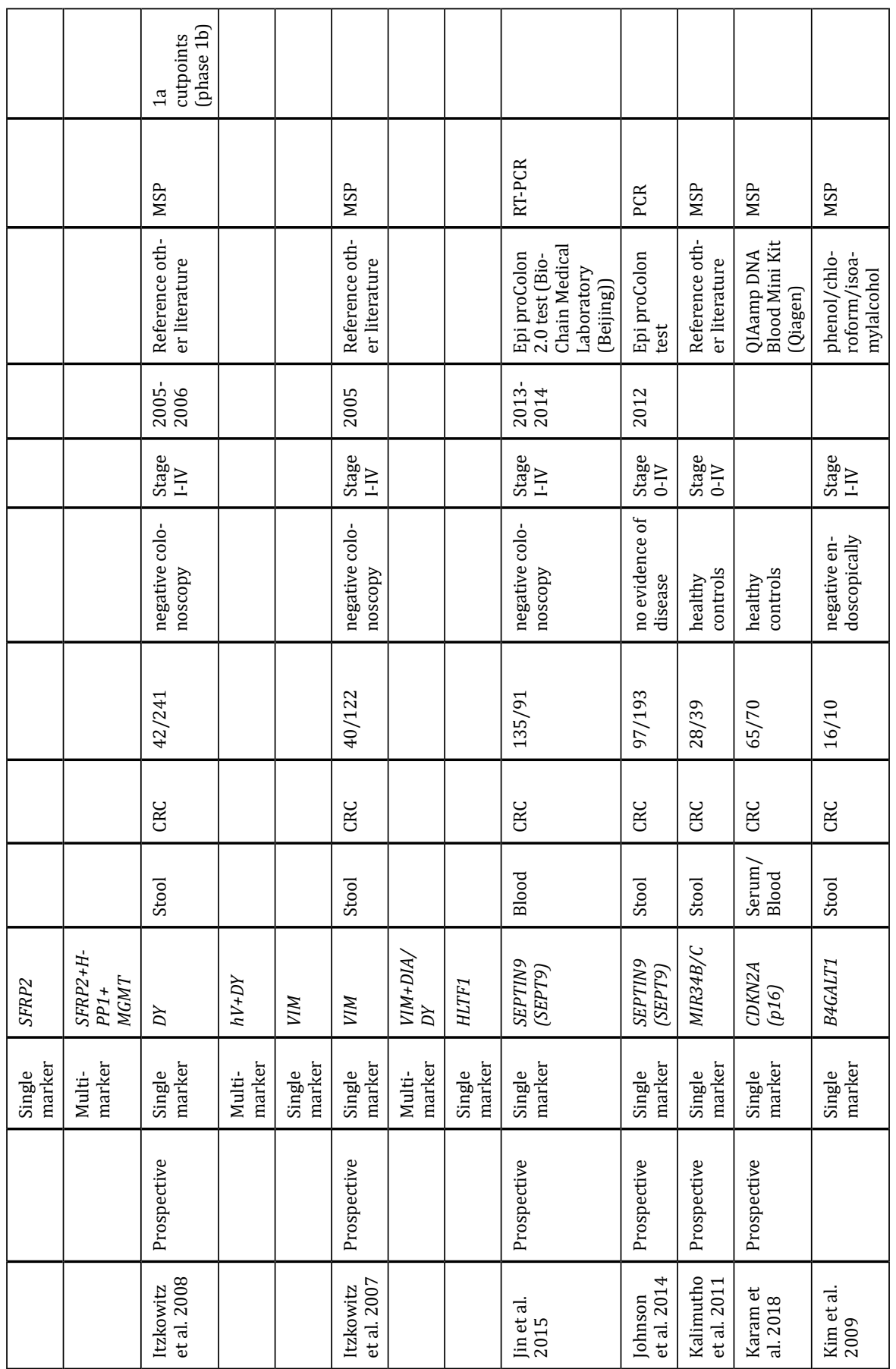




\begin{tabular}{|c|c|c|c|c|c|c|c|c|c|c|}
\hline & & & & & 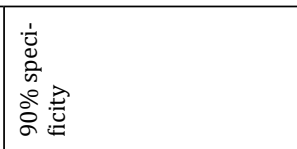 & & $\begin{array}{l}\infty \\
\infty \\
0 \\
0\end{array}$ & & & \\
\hline & & & & $\tilde{n}_{\Sigma}^{\bar{n}}$ & 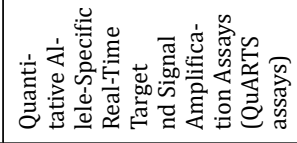 & $\sum_{\substack{\infty \\
\infty}}^{n}$ & & 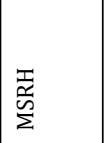 & 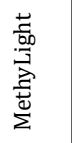 & \\
\hline & & & & 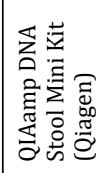 & 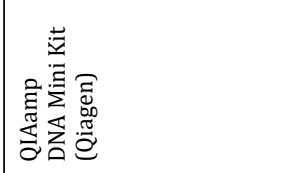 & & & & & 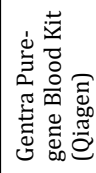 \\
\hline & & & & & 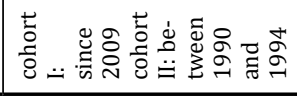 & 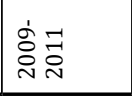 & & & & \\
\hline & & & 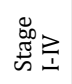 & & & 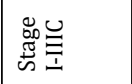 & & 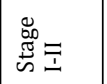 & & \\
\hline 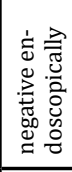 & & & 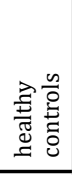 & 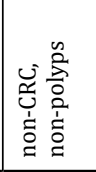 & 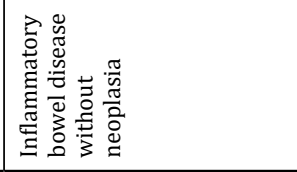 & 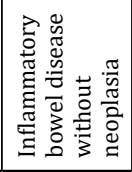 & & 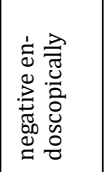 & & \\
\hline$\stackrel{\text { in }}{\stackrel{\text { in }}{2}}$ & & & $\begin{array}{l}\vec{\infty} \\
\hat{\sigma}\end{array}$ & 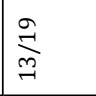 & & $\stackrel{\stackrel{n}{m}}{\frac{2}{2}}$ & & $\underset{\substack{N \\
\infty}}{\sim}$ & & 웅 \\
\hline & & & & Ư & 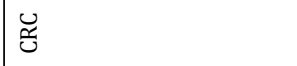 & Ư & & Ư & & ư \\
\hline & & & & $\vec{D}$ & $\begin{array}{l}\overline{8} \\
\dot{D} \\
\dot{\omega}\end{array}$ & $\overrightarrow{0}$ & & $\begin{array}{l}\overrightarrow{0} \\
\dot{0} \\
\dot{\omega}\end{array}$ & & $\begin{array}{l}\bar{\sigma} \\
\stackrel{\circ}{\infty}\end{array}$ \\
\hline 今̃ & 帘 & 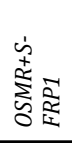 & ڤั & 蓄 & 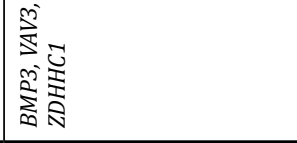 & $\sum_{\infty}^{\infty}$ & 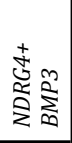 & \begin{tabular}{|l}
$\tilde{N}$ \\
空 \\
$\omega$
\end{tabular} & 逽 & 竎 \\
\hline 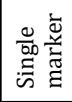 & 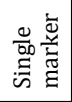 & 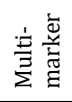 & 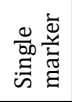 & 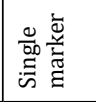 & 主 & 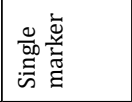 & 咅离 & 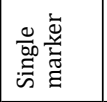 & 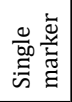 & 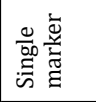 \\
\hline & & & & 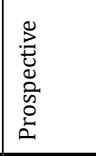 & 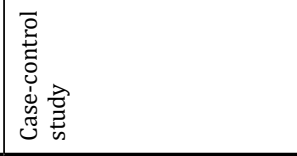 & 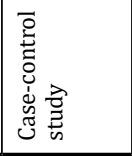 & & 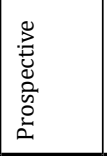 & & 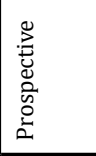 \\
\hline & & & & 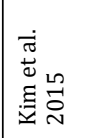 & 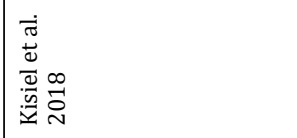 & 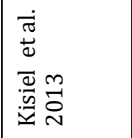 & & 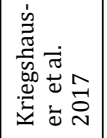 & & 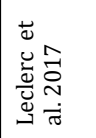 \\
\hline
\end{tabular}


DIAGNOSTIC DNA METHYLATION BIOMARKERS

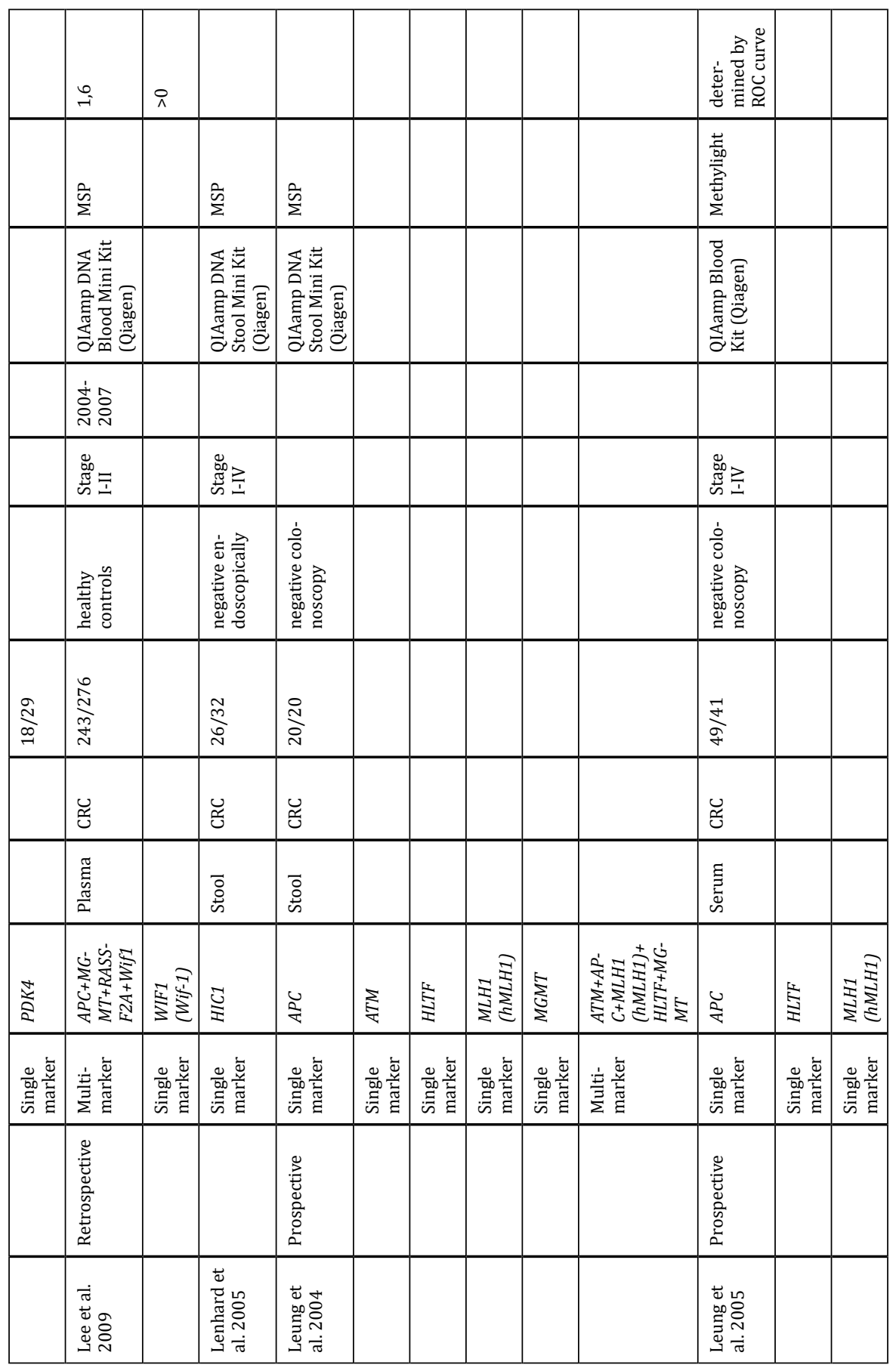




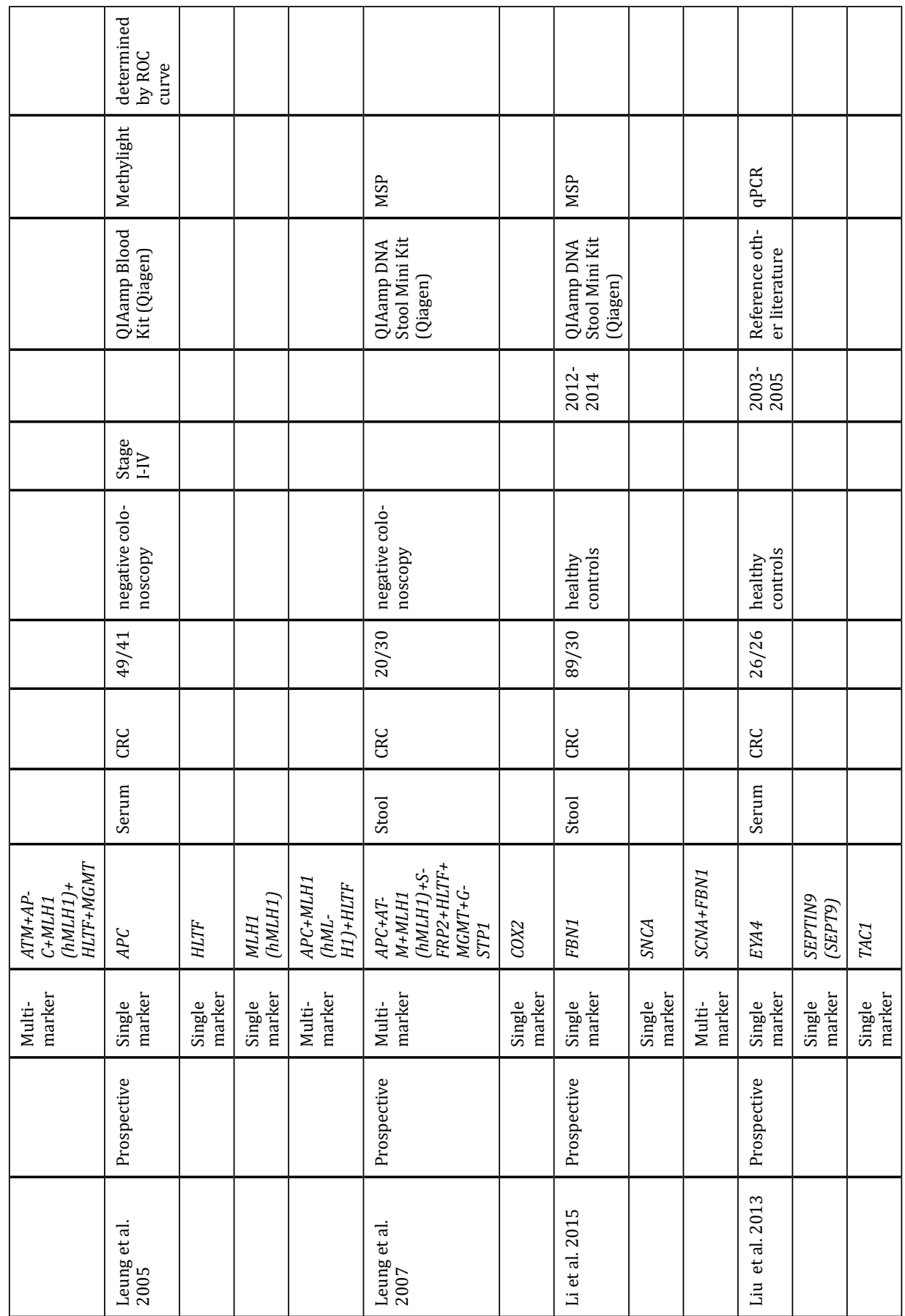


DIAGNOSTIC DNA METHYLATION BIOMARKERS

\begin{tabular}{|c|c|c|c|c|c|c|c|c|c|c|}
\hline & & 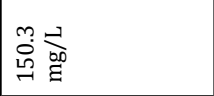 & : & $\vec{F}$ & $\mid \begin{array}{ll}\infty & 2 \\
0 \\
0 \\
0 & 0.0\end{array}$ & & & & & \\
\hline & & 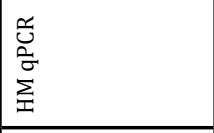 & & & & & & 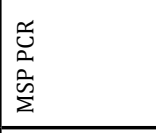 & & \\
\hline & & 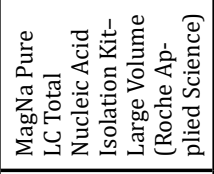 & & & & & & 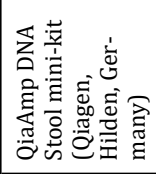 & & \\
\hline & & & & & & & & 啇 & & \\
\hline & & 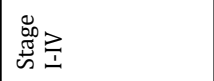 & & & & & & 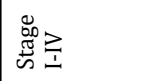 & & \\
\hline & & 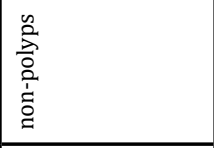 & & & & & & & & \\
\hline & & 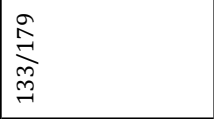 & & & & & & 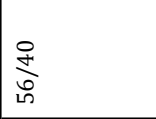 & & \\
\hline & & $\ddot{\mathscr{Z}}$ & & & & & & $\ddot{\sharp}$ & & \\
\hline & & 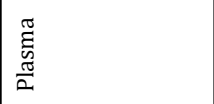 & & & & & & $\begin{array}{l}\overrightarrow{8} \\
\stackrel{8}{\circ} \\
\dot{\omega}\end{array}$ & & \\
\hline 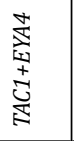 & 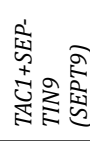 & 齐 & 莣 & 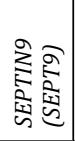 & 黄 & 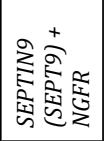 & 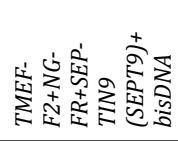 & 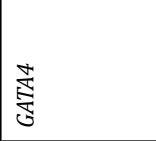 & 量 & 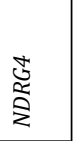 \\
\hline 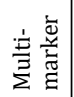 & 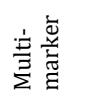 & 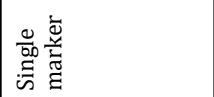 & 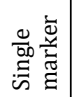 & 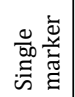 & 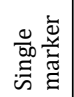 & 竞咅 & 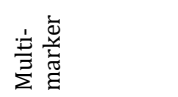 & 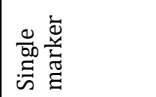 & 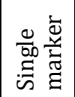 & 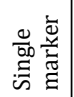 \\
\hline & & 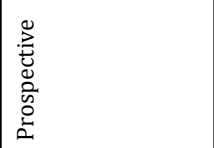 & & & & & & 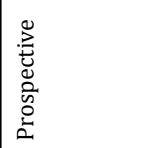 & & \\
\hline & & 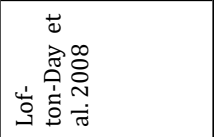 & & & & & & 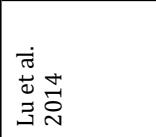 & & \\
\hline
\end{tabular}


CHAPTER 2

\begin{tabular}{|c|c|c|c|c|c|c|c|c|c|c|c|c|}
\hline & & & & & & & & & & & $\underset{\sim}{\sim}$ & $\underset{\sim}{\mathbb{N}}$ \\
\hline & & & 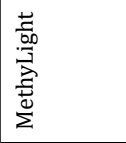 & 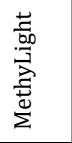 & 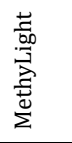 & 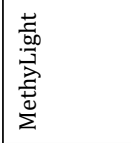 & 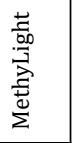 & 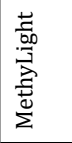 & 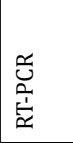 & & $\sum_{i}^{\infty}$ & $\sum_{i}^{\infty}$ \\
\hline & & & 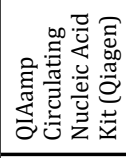 & & & 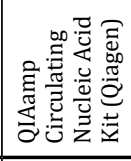 & & & & & & \\
\hline & & & 宫 & & & ôj & & & $\begin{array}{l}\dot{b} \\
\stackrel{2}{\sigma} \\
\sigma \\
\sigma\end{array}$ & & & \\
\hline & & & & & & & & & & & 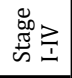 & 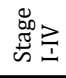 \\
\hline & & & & & & & & & & & 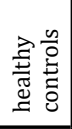 & 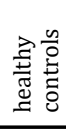 \\
\hline & & & $\frac{8}{2}$ & & & 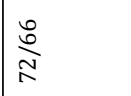 & & & 离 & & 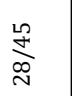 & 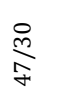 \\
\hline & & & 疍 & & & ư & & & 兄 & 寽 & 寽 & 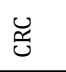 \\
\hline & & & 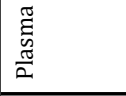 & & & 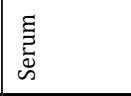 & & & $\begin{array}{l}\overrightarrow{8} \\
\dot{0} \\
\dot{m}\end{array}$ & 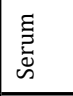 & $\begin{array}{l}\overline{0} \\
\text { 品 }\end{array}$ & \\
\hline 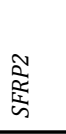 & $\underset{\Xi}{\vdots}$ & 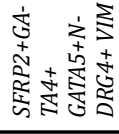 & 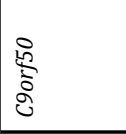 & 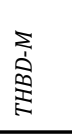 & 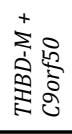 & לํ. & 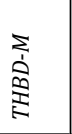 & 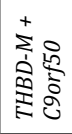 & $\sum_{i=1}^{i}$ & $\sum_{i=1}^{i}$ & $\begin{array}{l}\text { J్t } \\
\vdots \\
\vdots \\
\vdots\end{array}$ & \begin{tabular}{l}
\multirow{J}{*}{} \\
$\vdots$ \\
$\vdots$ \\
$\vdots$
\end{tabular} \\
\hline 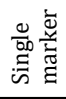 & 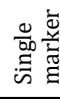 & 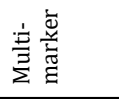 & 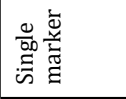 & 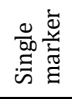 & 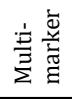 & 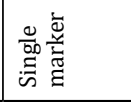 & 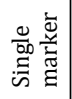 & 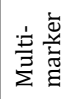 & 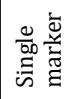 & 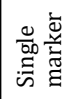 & 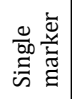 & 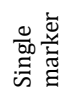 \\
\hline & & & 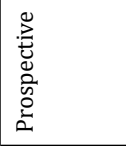 & & & & & & 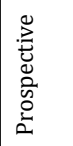 & & 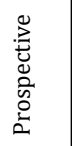 & \\
\hline & & & 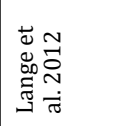 & & & & & & 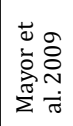 & & 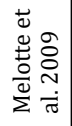 & \\
\hline
\end{tabular}


DIAGNOSTIC DNA METHYLATION BIOMARKERS

\begin{tabular}{|c|c|c|c|c|c|c|c|c|c|c|c|}
\hline 0 & 0 & 0 & 0 & 0 & 0 & & & & & & \\
\hline$\sum_{i}^{\infty}$ & & & & & $\sum_{i}^{\infty}$ & 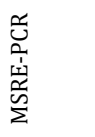 & & & & 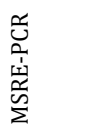 & 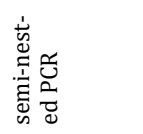 \\
\hline 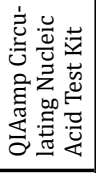 & & & & & 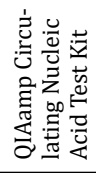 & 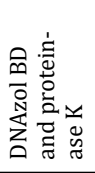 & & & & 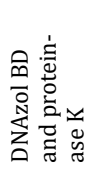 & 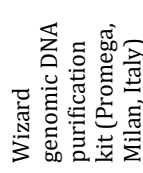 \\
\hline 今े & & & & & 今े & & & & & & \\
\hline 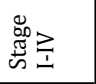 & & & & & 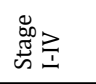 & $\begin{array}{l}\text { 品 } \\
\text { 总 }\end{array}$ & & & & 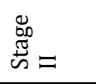 & \\
\hline 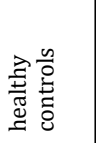 & & & & & 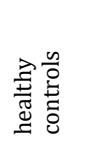 & 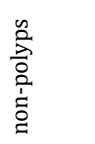 & & & & 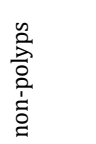 & 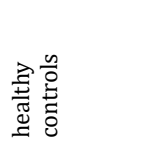 \\
\hline 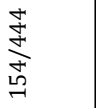 & & & & & 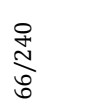 & 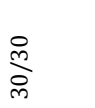 & & & & & 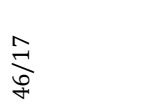 \\
\hline પ્ષ & & & & & 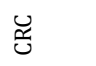 & 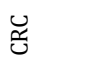 & & & & ્ֶّ & ̛̊ \\
\hline $\begin{array}{l}\frac{\pi}{\tilde{g}} \\
\frac{\pi}{\pi} \\
\frac{\pi}{2}\end{array}$ & & & & & $\begin{array}{l}\pi \\
\widetilde{\Xi} \\
\tilde{\pi} \\
\frac{\pi}{2}\end{array}$ & $\begin{array}{l}\underset{\pi}{\tilde{n}} \\
\frac{\pi}{\sim} \\
\frac{\pi}{2}\end{array}$ & & & & & $\begin{array}{l}\underset{\pi}{\Xi} \\
\frac{\pi}{2} \\
\frac{\pi}{2}\end{array}$ \\
\hline 丞 & 苞 & 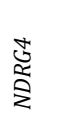 & 焉 & 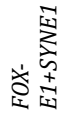 & 焉 & 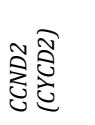 & 住 & $\vec{\Xi}$ & 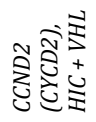 & 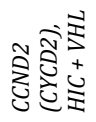 & $\stackrel{ \pm}{8}$ \\
\hline 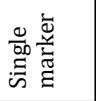 & 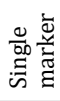 & 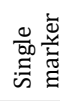 & 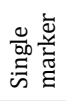 & 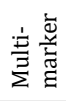 & 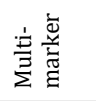 & 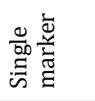 & 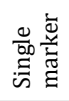 & 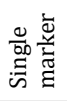 & 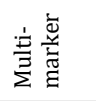 & 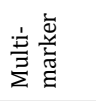 & 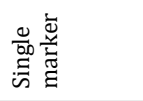 \\
\hline 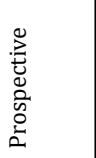 & & & & & & 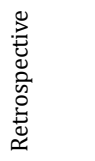 & & & & & 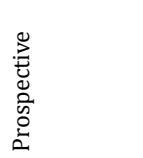 \\
\hline 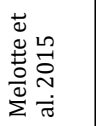 & & & & & & 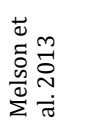 & & & & & 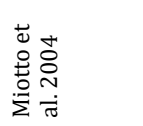 \\
\hline
\end{tabular}


CHAPTER 2

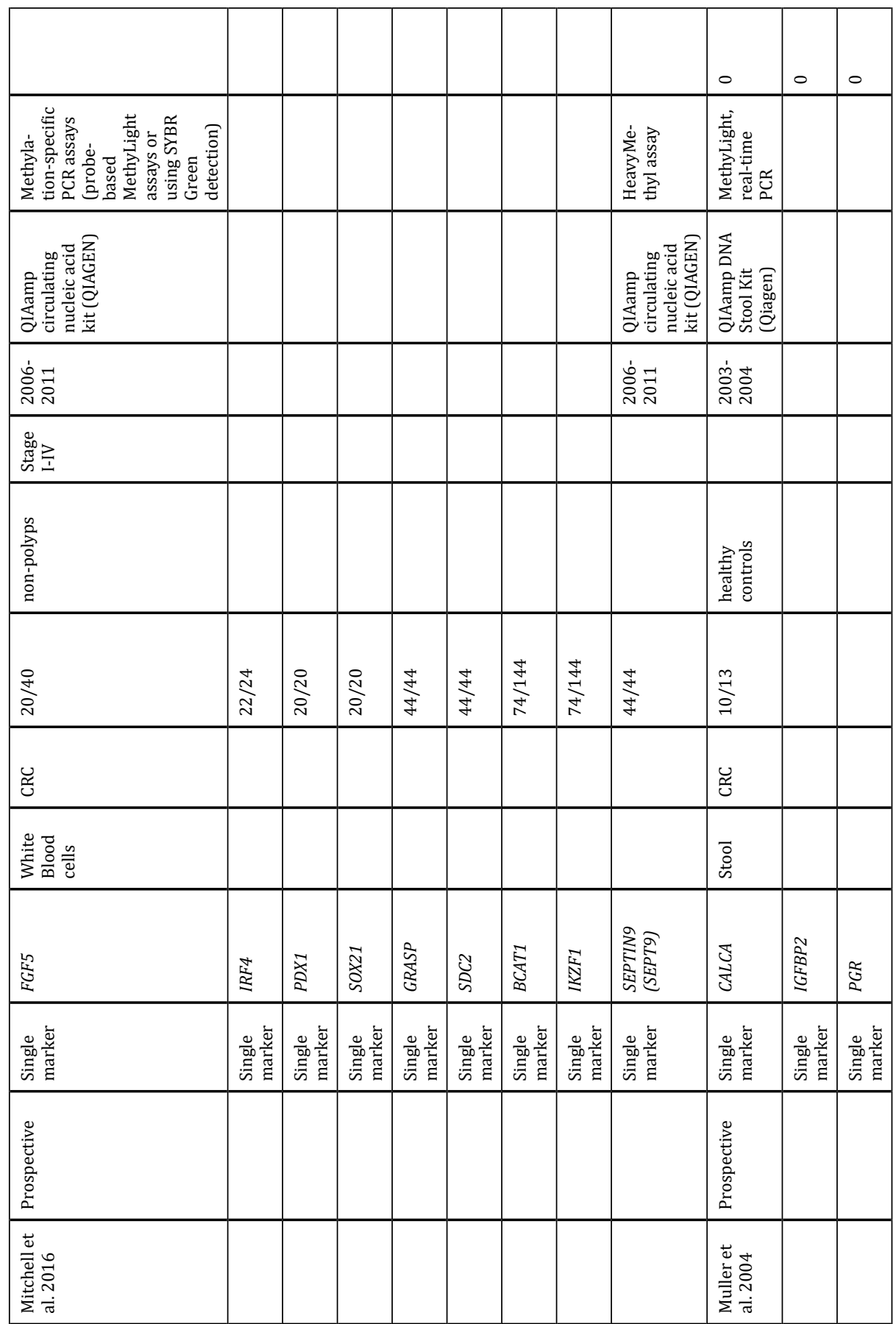


DIAGNOSTIC DNA METHYLATION BIOMARKERS

\begin{tabular}{|c|c|c|c|c|c|c|c|c|c|c|c|c|}
\hline 0 & 0 & 0 & 0 & 0 & 0 & 0 & 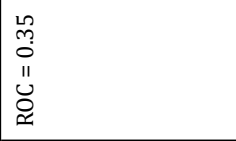 & & & & & \\
\hline & & 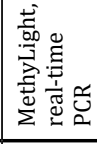 & & & & & 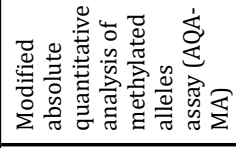 & 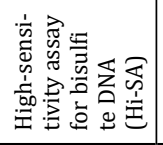 & & & & \\
\hline & & 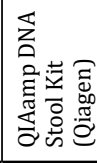 & & & & & 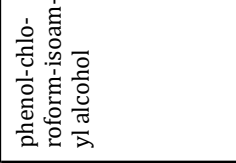 & 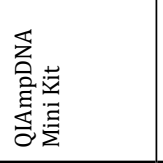 & & & & \\
\hline & & 悹䓂 & & & & & 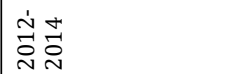 & 荾商 & & & & \\
\hline & & & & & & & 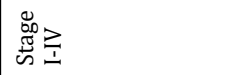 & 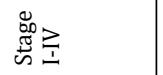 & & & & \\
\hline & & 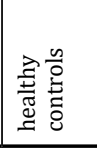 & & & & & 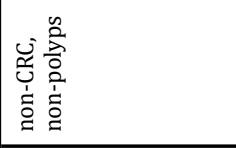 & 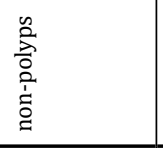 & & & & \\
\hline & & 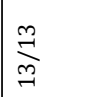 & & & & & $\begin{array}{l}\stackrel{m}{\rho} \\
\stackrel{f}{F}\end{array}$ & $\underset{\infty}{\stackrel{m}{\infty}}$ & & & & \\
\hline & & : & & & & & 岇 & ت্ّ & & & & \\
\hline & & & & & & & $\mid$\begin{tabular}{|l}
$\frac{\pi}{5}$ \\
$\frac{\pi}{2}$ \\
$\frac{\pi}{2}$
\end{tabular} & $\begin{array}{l}\overline{8} \\
\dot{8} \\
\dot{\omega}\end{array}$ & & & & \\
\hline 产 & 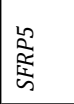 & 㥑 & 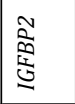 & 怘 & 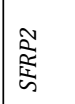 & 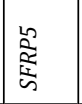 & 岀 & 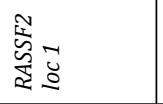 & 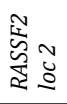 & 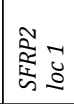 & 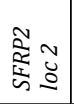 & $\mid$\begin{tabular}{|c|}
$\mid \widetilde{z}$ \\
$\overline{\tilde{z}}$ \\
\end{tabular} \\
\hline 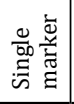 & 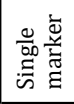 & 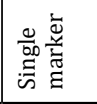 & 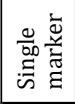 & 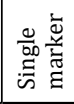 & 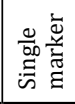 & 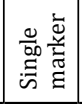 & 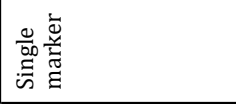 & 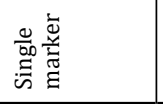 & 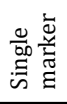 & 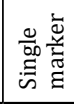 & 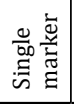 & \\
\hline & & & & & & & 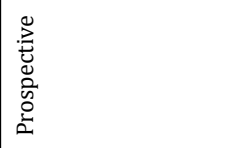 & 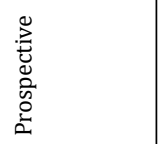 & & & & \\
\hline & & & & & & & 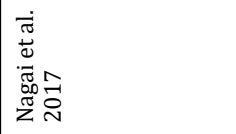 & 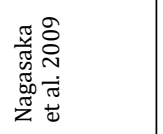 & & & & \\
\hline
\end{tabular}


CHAPTER 2

\begin{tabular}{|c|c|c|c|c|c|c|c|c|}
\hline 离 & 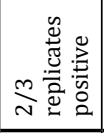 & 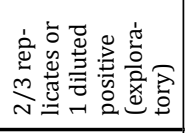 & & & & & 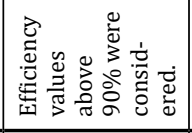 & \\
\hline $\begin{array}{c}\text { 品 } \\
\end{array}$ & & & 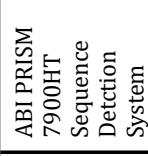 & 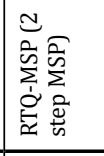 & 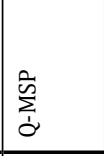 & & 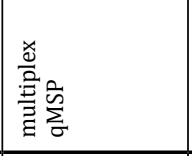 & \\
\hline 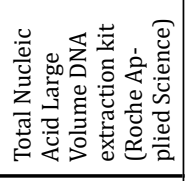 & & & & 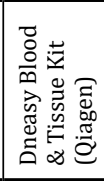 & 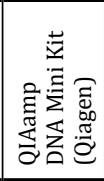 & & 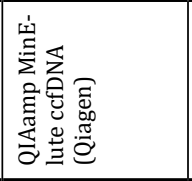 & \\
\hline & & & 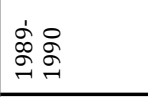 & & 豙虽 & & & \\
\hline $\begin{array}{l}\text { 总 } \\
\text { 岕 }\end{array}$ & $\begin{array}{l}\text { 总 } \\
\text { 恖 } \\
\dot{w}\end{array}$ & & & 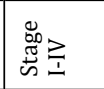 & 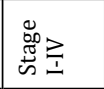 & 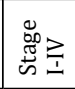 & 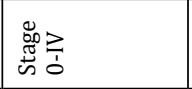 & \\
\hline & & & 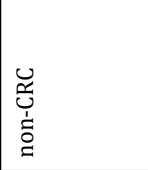 & 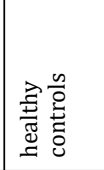 & 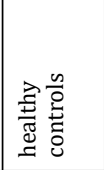 & 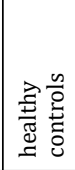 & 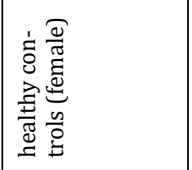 & \\
\hline$\underset{\substack{\sim \\
\stackrel{\sim}{\sim}}}{\sim}$ & 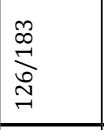 & & $\begin{array}{l}\vec{b} \\
\stackrel{0}{\infty} \\
\infty \\
0 \\
m \\
m\end{array}$ & $\begin{array}{l}\stackrel{0}{\circ} \\
\stackrel{2}{f} \\
\text { m }\end{array}$ & 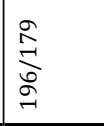 & 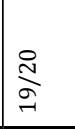 & $\underset{\substack{\infty\\
}}{\stackrel{0}{\mathbb{N}}}$ & \\
\hline ֶّ & $\ddot{\mathscr{U}}$ & & : & \begin{tabular}{|l}
$\ddot{U}$ \\
\end{tabular} & $\begin{array}{l}\mathscr{\varkappa} \\
\end{array}$ & & 䒕 & \\
\hline $\begin{array}{l}\vec{\Xi} \\
\frac{\circ}{\oplus}\end{array}$ & & & 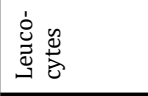 & 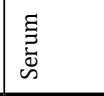 & 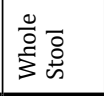 & $\overrightarrow{\mathrm{o}}$ & \begin{tabular}{|l}
$\overrightarrow{\text { og }}$ \\
品
\end{tabular} & \\
\hline 高 & 裹高 & 髉哀 & 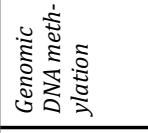 & 豙 & $\begin{array}{l}\tilde{U} \\
\tilde{\omega}\end{array}$ & $\begin{array}{l}\tilde{U} \\
\tilde{\omega}\end{array}$ & 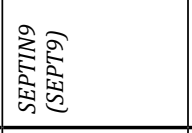 & $\ddot{z}$ \\
\hline 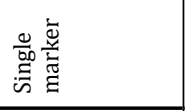 & 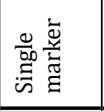 & 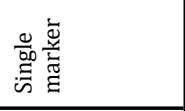 & & 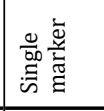 & 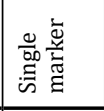 & 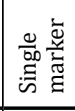 & 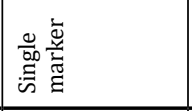 & 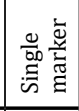 \\
\hline 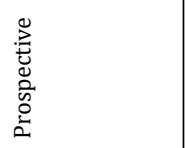 & & & 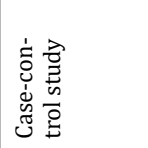 & 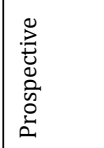 & 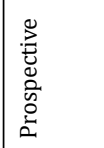 & & 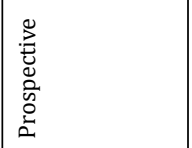 & \\
\hline 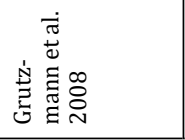 & & & 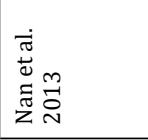 & 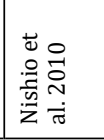 & 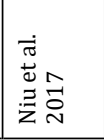 & & 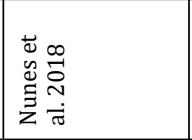 & \\
\hline
\end{tabular}


DIAGNOSTIC DNA METHYLATION BIOMARKERS

\begin{tabular}{|c|c|c|c|c|c|c|c|c|c|}
\hline & & & & & & 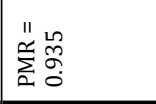 & $\begin{array}{l}0 \\
\tilde{D} \\
\tilde{U}\end{array}$ & 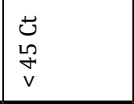 & 0 \\
\hline & & & & & & 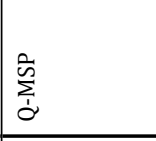 & 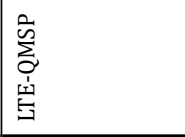 & 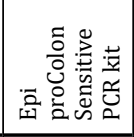 & 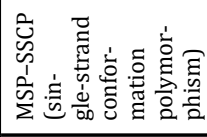 \\
\hline & & & & & & 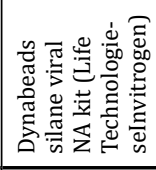 & 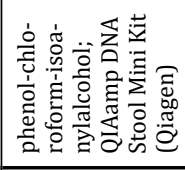 & 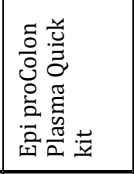 & \\
\hline & & & & & & & & 월 & \\
\hline & & & & & 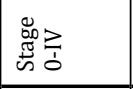 & 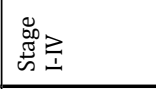 & 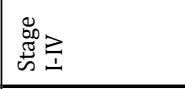 & 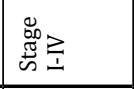 & 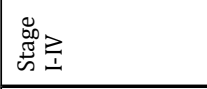 \\
\hline & & & & & 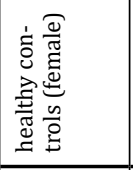 & 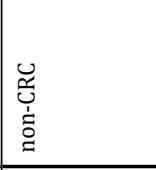 & 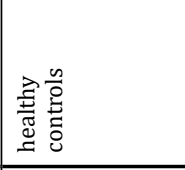 & 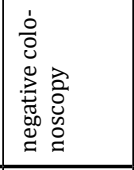 & 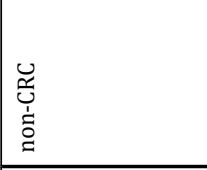 \\
\hline & & & & & 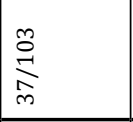 & 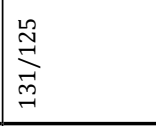 & 离 & 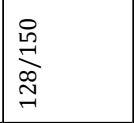 & $\begin{array}{l}0 \\
8 \\
0 \\
0\end{array}$ \\
\hline & & & & & & 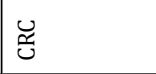 & ષ̈ & ্ֶّ & $\ddot{\forall}$ \\
\hline & & & & & & 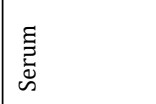 & $\begin{array}{l}\overline{\mathrm{g}} \\
\mathrm{d}\end{array}$ & \begin{tabular}{|l}
$\frac{\pi}{\tilde{E}}$ \\
$\frac{\pi}{\pi}$ \\
$\frac{\pi}{\pi}$
\end{tabular} & $\mid \begin{array}{l}\frac{\pi}{5} \\
\frac{\pi}{\pi} \\
\frac{\pi}{2}\end{array}$ \\
\hline 离 & $\begin{array}{l}\tilde{z} \\
\tilde{z} \\
\mathbb{z}\end{array}$ & 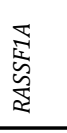 & 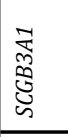 & 离 & 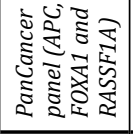 & ్ֶ & \begin{tabular}{|l}
$\tilde{U}$ \\
$\tilde{\omega}$ \\
\end{tabular} & 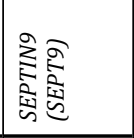 & $\frac{u}{2}$ \\
\hline 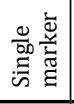 & 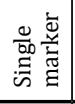 & 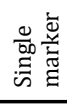 & 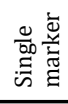 & 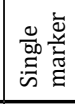 & $\begin{array}{l}\text { 訔离 } \\
\text { 离 } \\
\end{array}$ & 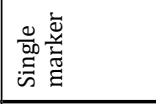 & 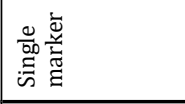 & 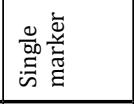 & 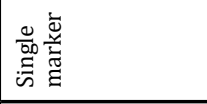 \\
\hline & & & & & & 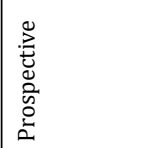 & 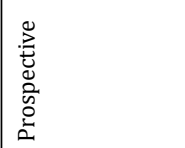 & 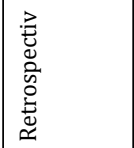 & 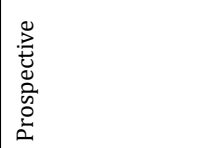 \\
\hline & & & & & & 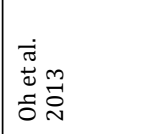 & 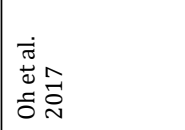 & 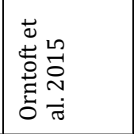 & 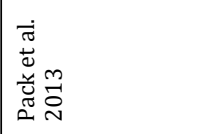 \\
\hline
\end{tabular}


CHAPTER 2

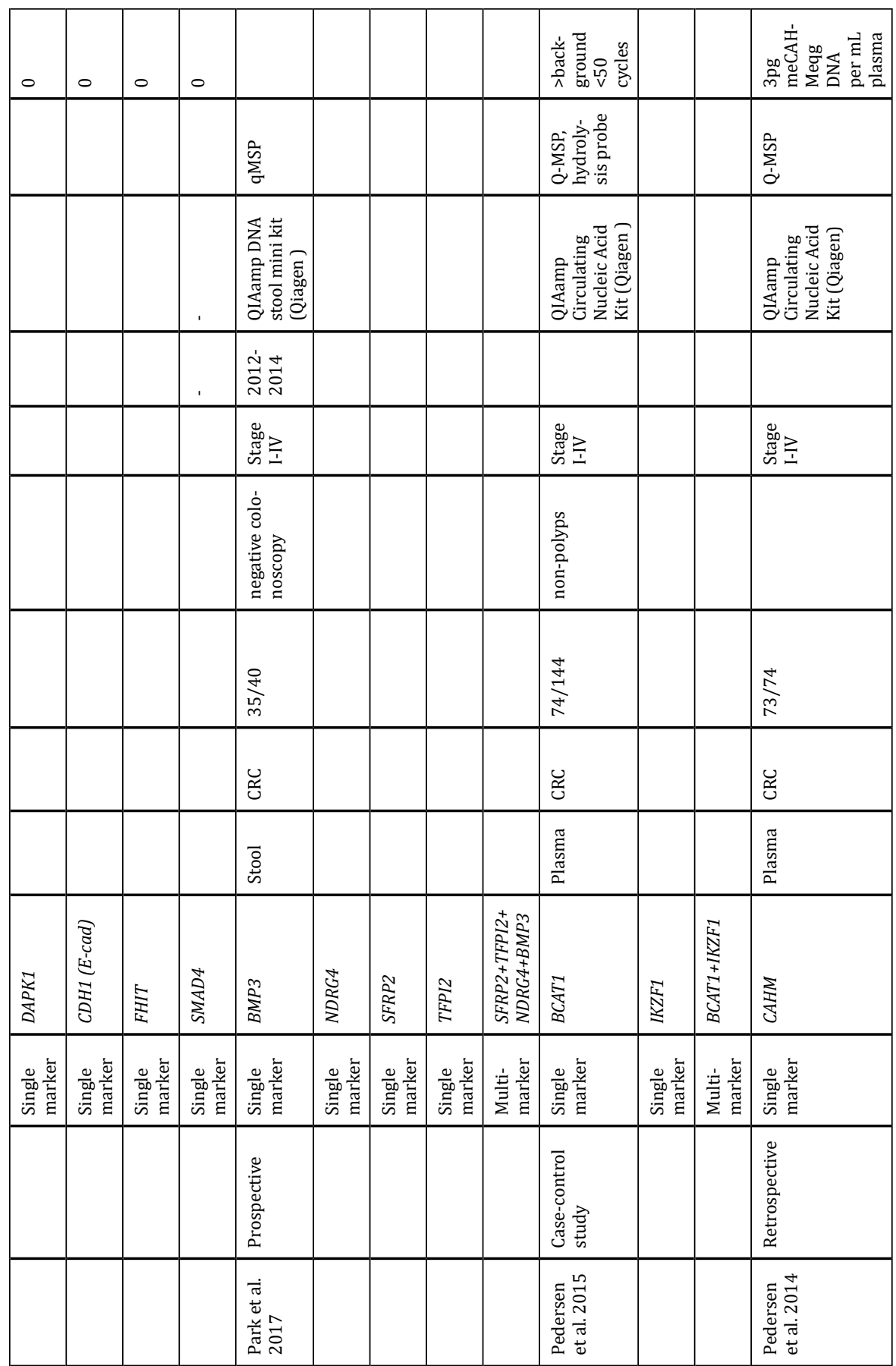


DIAGNOSTIC DNA METHYLATION BIOMARKERS

\begin{tabular}{|c|c|c|c|c|c|c|c|c|c|c|c|c|}
\hline 0 & 0 & & 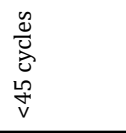 & 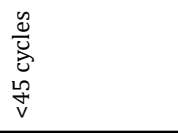 & 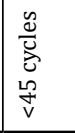 & 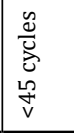 & 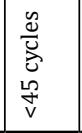 & 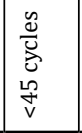 & 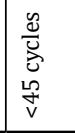 & 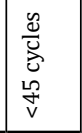 & 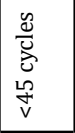 & 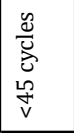 \\
\hline 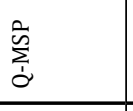 & & & 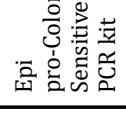 & 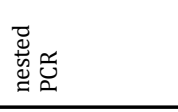 & & & & & & & & \\
\hline 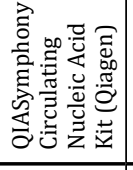 & & & 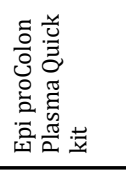 & 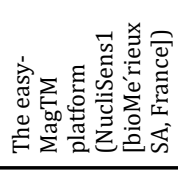 & & & & & & & & \\
\hline 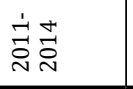 & & & & 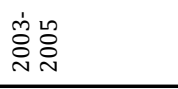 & & & & & & & & \\
\hline 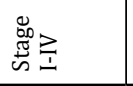 & & & 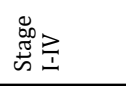 & 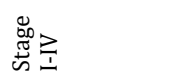 & & & & & & & & \\
\hline 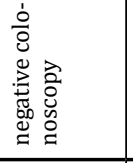 & & & 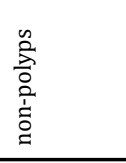 & 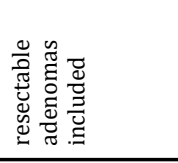 & & & & & & & & \\
\hline 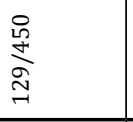 & & & $\frac{z}{\frac{7}{z}}$ & 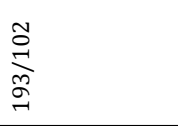 & & & & & & & & \\
\hline ्ֶञ & & & 过 & Æ̈ & & & & & & & & \\
\hline $\begin{array}{l}\frac{\pi}{5} \\
\frac{\pi}{2} \\
\frac{\pi}{2}\end{array}$ & & & 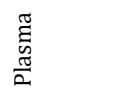 & $\begin{array}{l}\frac{\pi}{5} \\
\frac{\pi}{0} \\
\frac{\pi}{2}\end{array}$ & & & & & & & & \\
\hline 気 & $\begin{array}{l}\mathbb{Z} \\
:\end{array}$ & 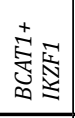 & 施高 & तै & 岁 & $\sum_{\substack{\infty \\
\Sigma}}^{\infty}$ & 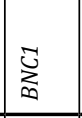 & 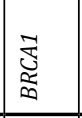 & 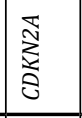 & 岕 & 留 & 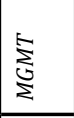 \\
\hline 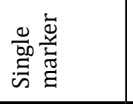 & 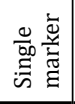 & 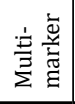 & 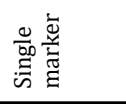 & 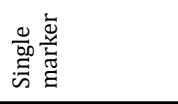 & 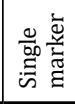 & 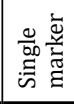 & 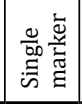 & 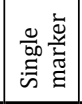 & 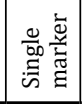 & 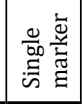 & 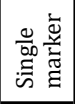 & 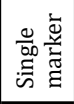 \\
\hline 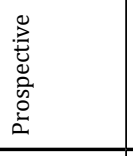 & & & 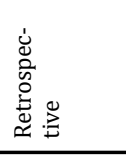 & 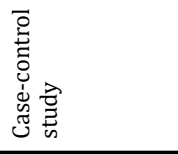 & & & & & & & & \\
\hline 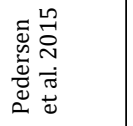 & & & 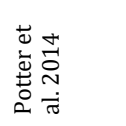 & 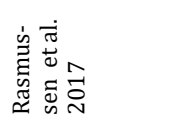 & & & & & & & & \\
\hline
\end{tabular}




\begin{tabular}{|c|c|c|c|c|c|c|c|c|c|c|c|c|c|c|c|c|c|c|c|c|c|c|}
\hline 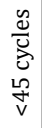 & 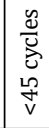 & 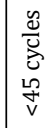 & 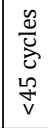 & 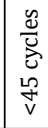 & 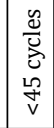 & 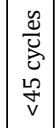 & 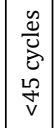 & 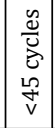 & 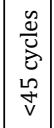 & 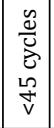 & 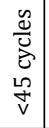 & 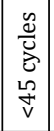 & 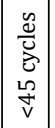 & 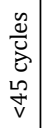 & 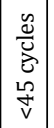 & 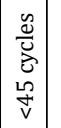 & 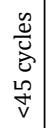 & 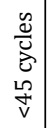 & 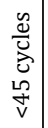 & 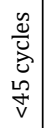 & & 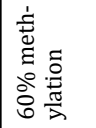 \\
\hline & & & & & & & & & & & & & & & & & & & & & & $\mid \begin{array}{l}0 \\
\sum_{0}^{n} \\
0\end{array}$ \\
\hline & & & & & & & & & & & & & & & & & & & & & & 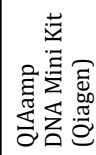 \\
\hline & & & & & & & & & & & & & & & & & & & & & & 节 \\
\hline & & & & & & & & & & & & & & & & & & & & & & 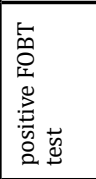 \\
\hline & & & & & & & & & & & & & & & & & & & & & & 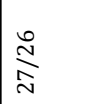 \\
\hline & & & & & & & & & & & & & & & & & & & & & & ư \\
\hline & & & & & & & & & & & & & & & & & & & & & & 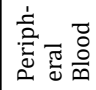 \\
\hline$\underset{I}{\Sigma}$ & 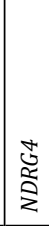 & 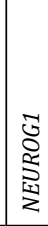 & 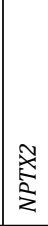 & క్ర & $\mid \begin{array}{l}m \\
\frac{2}{2} \\
\frac{2}{2} \\
\frac{\pi}{2}\end{array}$ & 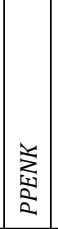 & $\mid \begin{array}{l}\infty \\
2 \\
2 \\
2\end{array}$ & 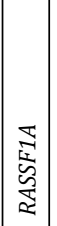 & जั & 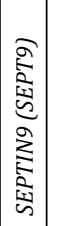 & 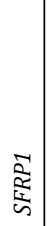 & $\mid$\begin{tabular}{|}
$\frac{1}{2}$ \\
$\frac{2}{5}$ \\
$\omega$
\end{tabular} & 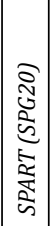 & $\widehat{\omega}$ & $\vec{U}$ & 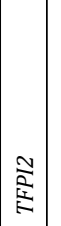 & 周 & 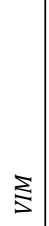 & $\stackrel{5}{3}$ & 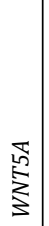 & 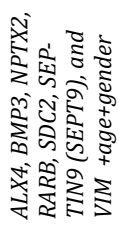 & 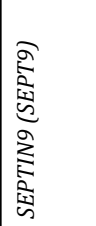 \\
\hline 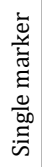 & 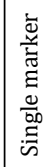 & 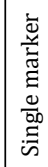 & 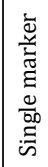 & 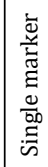 & 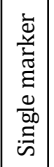 & 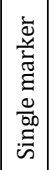 & 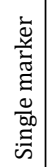 & 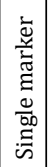 & 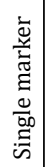 & 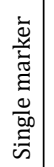 & 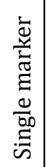 & 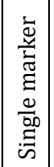 & 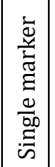 & 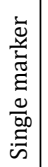 & 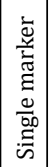 & 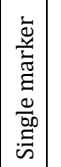 & 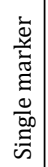 & 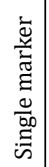 & 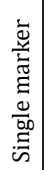 & 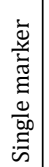 & 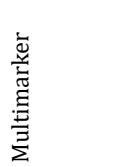 & 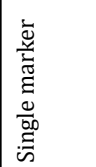 \\
\hline & & & & & & & & & & & & & & & & & & & & & & \\
\hline & & & & & & & & & & & & & & & & & & & & & & 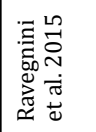 \\
\hline
\end{tabular}


DIAGNOSTIC DNA METHYLATION BIOMARKERS

\begin{tabular}{|c|c|c|c|c|c|c|c|c|c|}
\hline 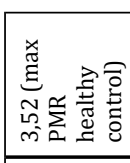 & 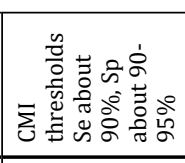 & & & & & & & 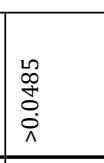 & \\
\hline 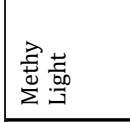 & $\begin{array}{l}0 \\
\sum_{n}^{n} \\
\sum_{0}^{1} \\
\end{array}$ & & & 离 & $\sum_{\Sigma}^{\tilde{n}}$ & $\frac{\bar{n}}{\Sigma}$ & $\frac{\hat{n}}{\Sigma}$ & 旀 & \\
\hline 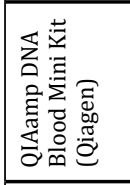 & 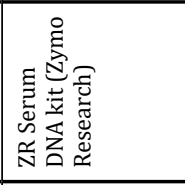 & & & 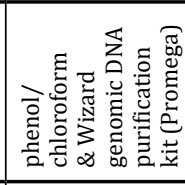 & 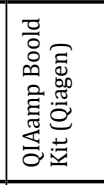 & 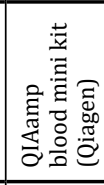 & 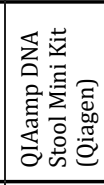 & 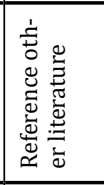 & \\
\hline \multicolumn{10}{|l|}{ 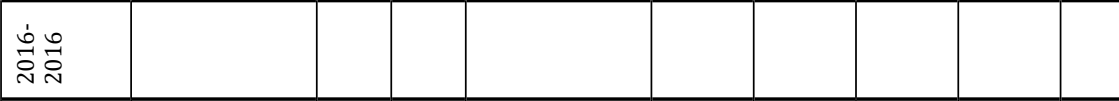 } \\
\hline & 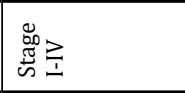 & & & & 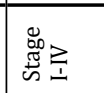 & & & 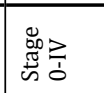 & \\
\hline 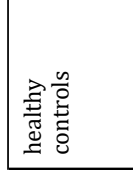 & 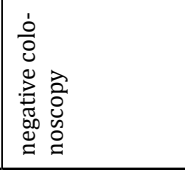 & 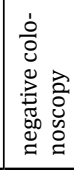 & 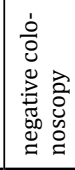 & 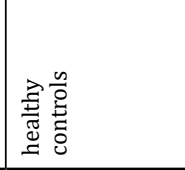 & 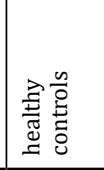 & 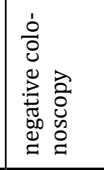 & 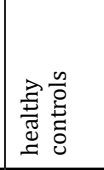 & 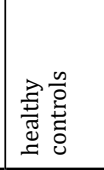 & \\
\hline$\underset{m}{\stackrel{m}{i}}$ & $\begin{array}{l}0 \\
\sigma \\
\sigma\end{array}$ & $\underset{\sim}{\vec{m}}$ & \begin{tabular}{|l}
$\vec{b}$ \\
$\stackrel{\vec{N}}{m}$ \\
$m$
\end{tabular} & 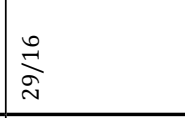 & in & $\stackrel{\substack{\mathcal{N} \\
\stackrel{2}{N}}}{N}$ & $\stackrel{\stackrel{2}{N}}{\stackrel{N}{N}}$ & 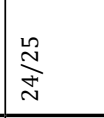 & \\
\hline ্ֶֶ & ש̈ & & & খ্ّ & 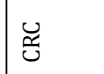 & : & : & Ư & \\
\hline $\begin{array}{l}\frac{\pi}{\tilde{E}} \\
\frac{\pi}{2} \\
\frac{\pi}{2}\end{array}$ & 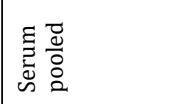 & & & 言 & E & 咅 & 总 & 部 & \\
\hline 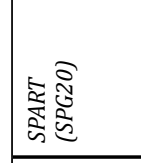 & $\frac{a}{z}$ & 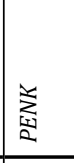 & 5 & 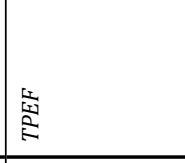 & 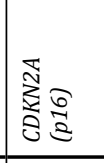 & 䓠 & 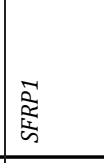 & ミ & 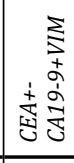 \\
\hline 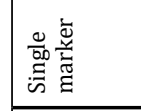 & 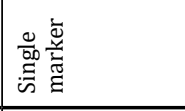 & 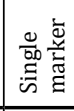 & 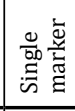 & 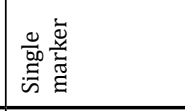 & 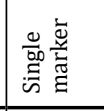 & 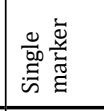 & 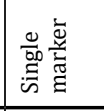 & 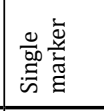 & 主 \\
\hline 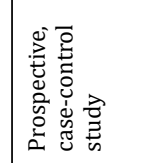 & 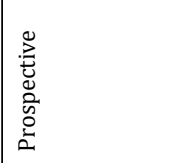 & & & 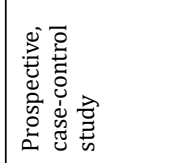 & 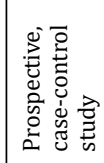 & 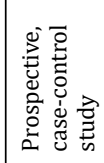 & 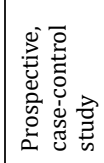 & 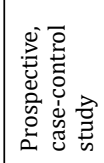 & \\
\hline 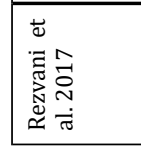 & 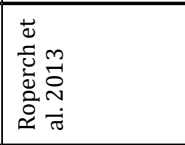 & & & 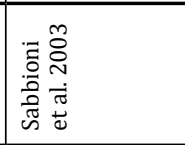 & 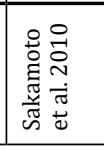 & 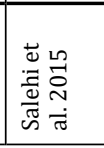 & 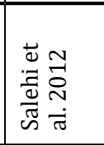 & 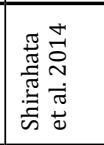 & \\
\hline
\end{tabular}




\begin{tabular}{|c|c|c|c|c|c|c|c|c|c|c|c|}
\hline & 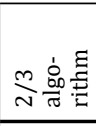 & $\stackrel{\substack{\mathscr{0} \\
\overline{0}}}{\stackrel{0}{0}}$ & 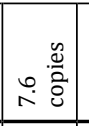 & 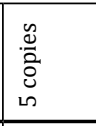 & & & & & & & \\
\hline 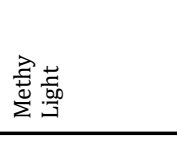 & 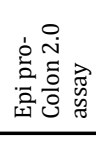 & 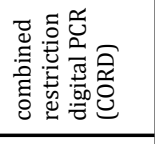 & & 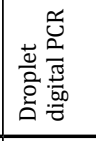 & \begin{tabular}{|l}
$\hat{n}$ \\
$\sum$
\end{tabular} & $\frac{n}{\Sigma}$ & 产 & & & $\frac{\tilde{n}}{\Sigma}$ & \\
\hline 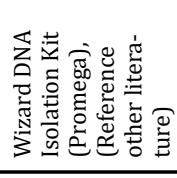 & 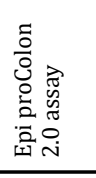 & 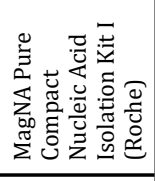 & & 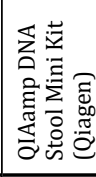 & 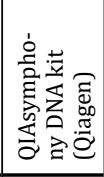 & 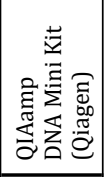 & 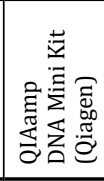 & & & 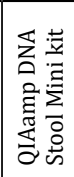 & 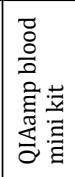 \\
\hline & & 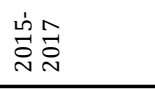 & & & 官芒 & & & & & & \\
\hline 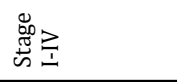 & 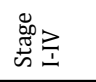 & 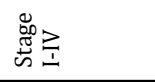 & & 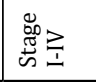 & 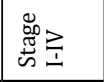 & 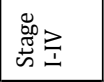 & & & & 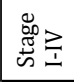 & 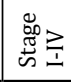 \\
\hline 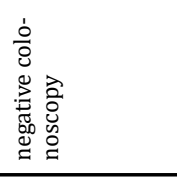 & 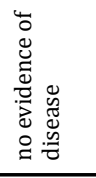 & 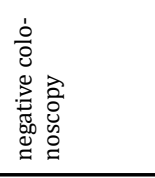 & & 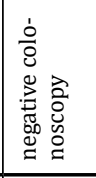 & 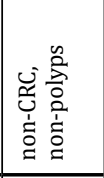 & 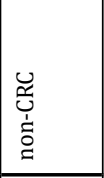 & & & & 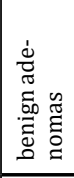 & 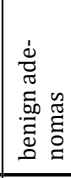 \\
\hline$\stackrel{\text { ì }}{\stackrel{N}{-}}$ & 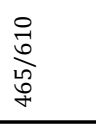 & 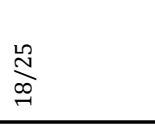 & & $\underset{\infty}{\stackrel{\infty}{\infty}}$ & \begin{tabular}{|l}
$n$ \\
$\stackrel{n}{2}$ \\
$\substack{n \\
0}$ \\
0
\end{tabular} & 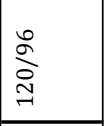 & & & & \begin{tabular}{|l}
0 \\
0 \\
0 \\
0 \\
0 \\
\end{tabular} & $\begin{array}{l}n \\
0 \\
0 \\
6 \\
- \\
\end{array}$ \\
\hline ษ્ّ & ֶّ & ֶّ & & $\ddot{\mathscr{U}}$ & Æّ̈ & ت̈ & & & & ت̈ & 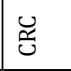 \\
\hline 总 & $\begin{array}{l}\tau \\
\frac{0}{m} \\
\end{array}$ & ह气 & & \begin{tabular}{|l}
$\overrightarrow{0}$ \\
$\dot{0}$ \\
\end{tabular} & 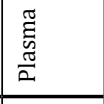 & 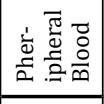 & & & & \begin{tabular}{|l}
$\overline{\mathrm{o}}$ \\
$\dot{\mathrm{s}}$ \\
\end{tabular} & 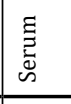 \\
\hline$\leqq$ & 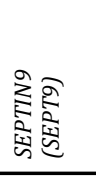 & 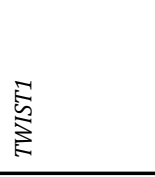 & 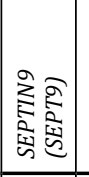 & E् & 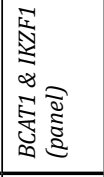 & 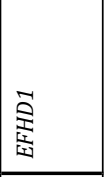 & \begin{tabular}{|l}
0 \\
0 \\
$\vdots$ \\
$\vdots$ \\
$\vdots$ \\
$\vdots$ \\
\end{tabular} & 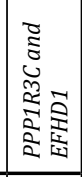 & 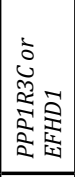 & 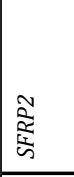 & 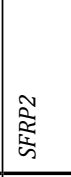 \\
\hline 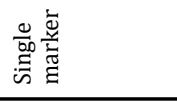 & 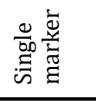 & 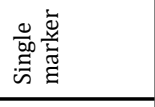 & 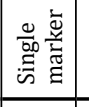 & 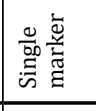 & 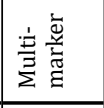 & 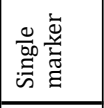 & 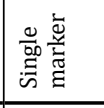 & 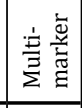 & 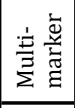 & 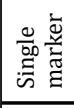 & 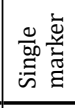 \\
\hline 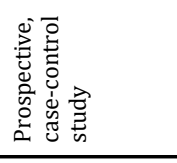 & 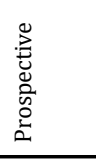 & & & 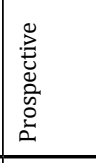 & 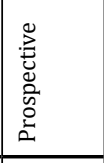 & 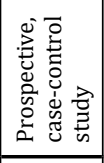 & & & & 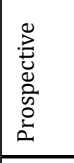 & \\
\hline 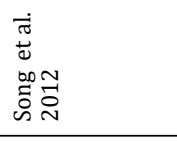 & 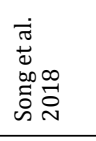 & 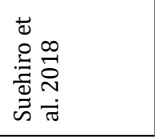 & & 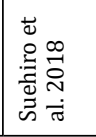 & 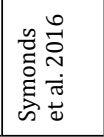 & 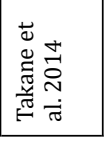 & & & & 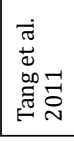 & \\
\hline
\end{tabular}


DIAGNOSTIC DNA METHYLATION BIOMARKERS

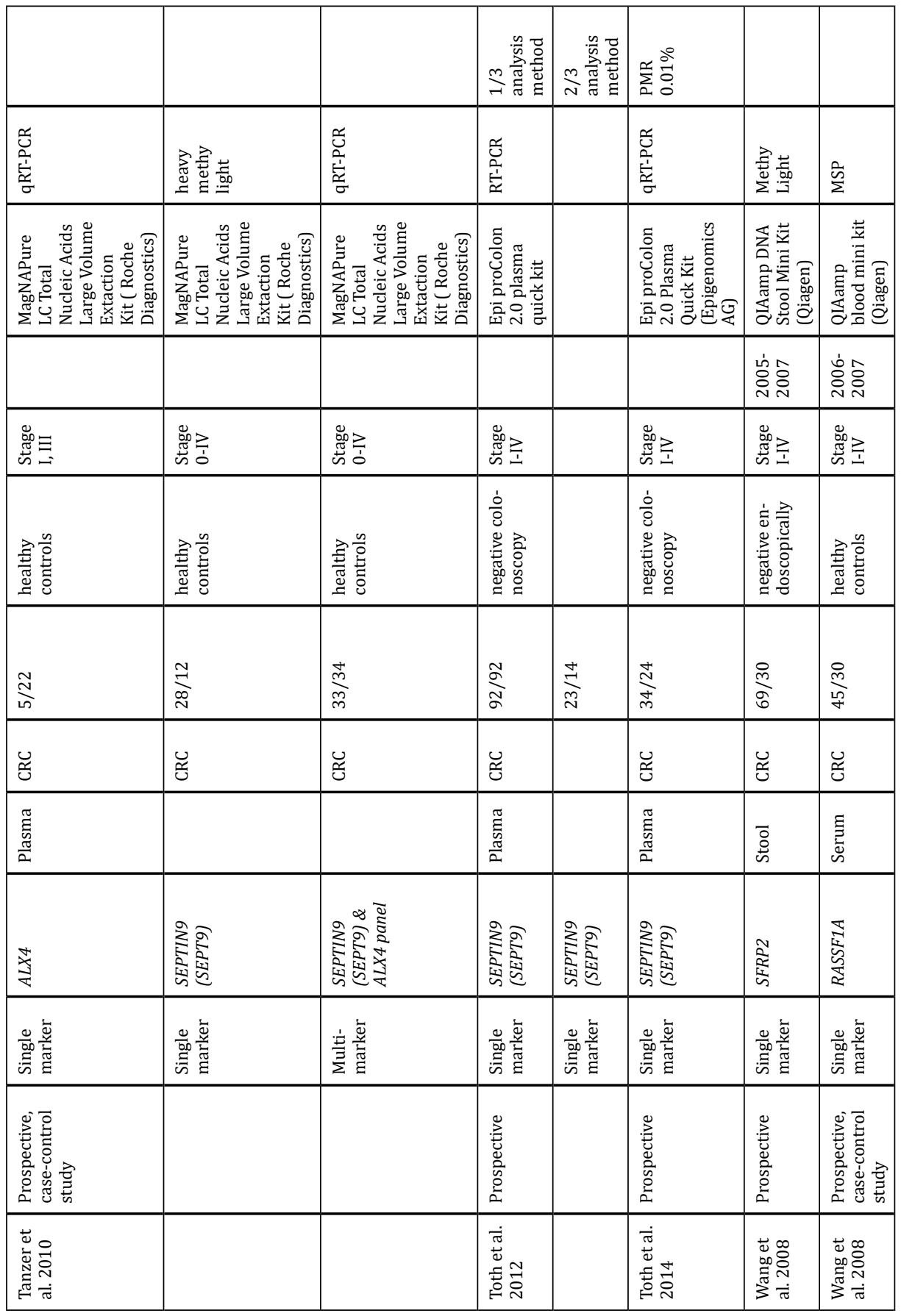


CHAPTER 2

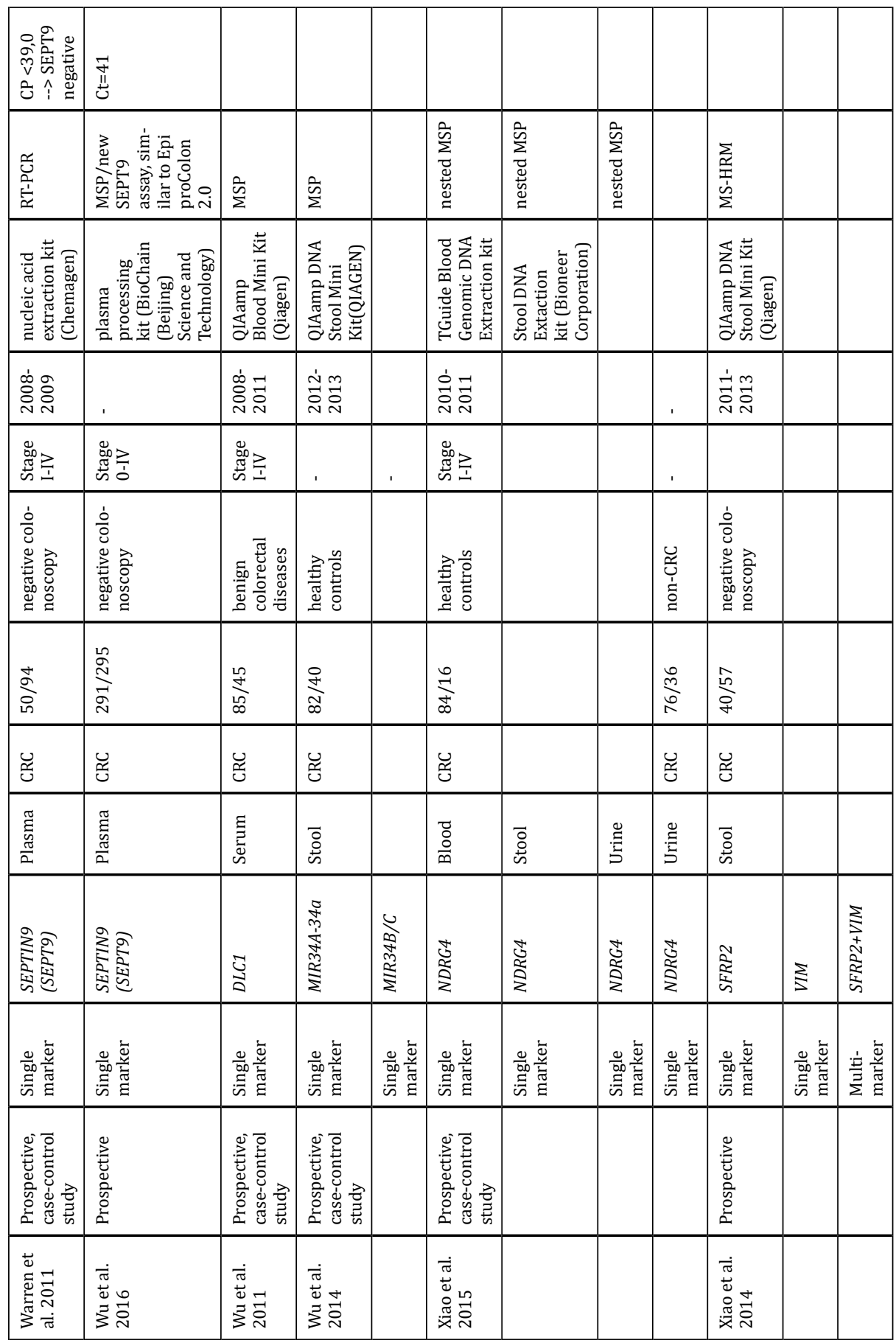




\begin{tabular}{|c|c|c|c|c|c|c|c|c|c|c|c|c|}
\hline תֶّ & & & & & & & & & & & & \\
\hline$\frac{\tilde{n}}{\sum}$ & $\sum$ & $\sum \bar{n}$ & 离 & $\frac{\hat{n}}{\Sigma}$ & $\frac{\hat{n}}{\Sigma}$ & & & & & & & \\
\hline 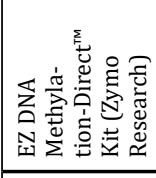 & 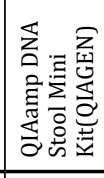 & 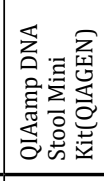 & & 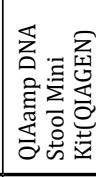 & 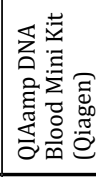 & & & & & & & \\
\hline 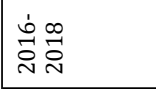 & & 官 & 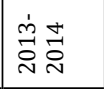 & & 로 & & & & & & & \\
\hline 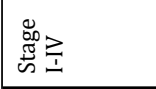 & 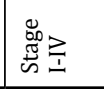 & 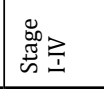 & 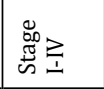 & 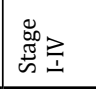 & 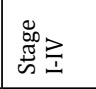 & & & & & & & \\
\hline 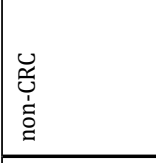 & 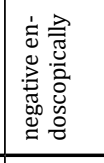 & 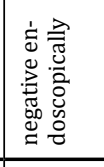 & 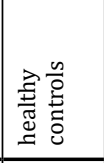 & 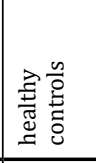 & 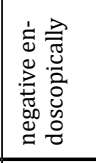 & & & & & & & \\
\hline 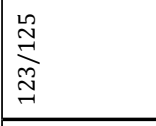 & 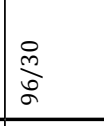 & \begin{tabular}{|l}
0 \\
o. \\
0 \\
0
\end{tabular} & \begin{tabular}{|l}
$\stackrel{0}{1}$ \\
in \\
in
\end{tabular} & $\stackrel{\stackrel{2}{2}}{\underset{2}{2}}$ & 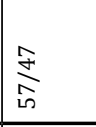 & & & & & & & \\
\hline$\ddot{\mathscr{U}}$ & : & Ü & ્ֶఝ & $\ddot{\mathscr{y}}$ & $\ddot{\ddot{y}}$ & & & & & & & \\
\hline \begin{tabular}{|l|}
$\frac{\pi}{5}$ \\
$\frac{\pi}{2}$ \\
$\frac{\pi}{2}$
\end{tabular} & $\begin{array}{l}\overline{0} \\
\dot{8} \\
\dot{\omega}\end{array}$ & \begin{tabular}{|l}
$\overline{0}$ \\
$\dot{8}$ \\
$\dot{n}$
\end{tabular} & $\begin{array}{l}\tilde{\delta} \\
\frac{\mathrm{o}}{\infty}\end{array}$ & $\begin{array}{l}\overline{0} \\
\dot{d}\end{array}$ & 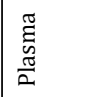 & & & & & & & \\
\hline 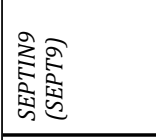 & 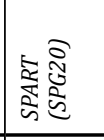 & \begin{tabular}{|l} 
产 \\
胥 \\
\end{tabular} & 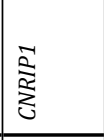 & 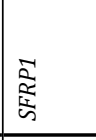 & 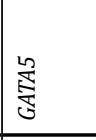 & & 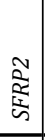 & 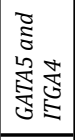 & 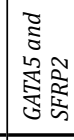 & 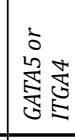 & 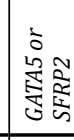 & 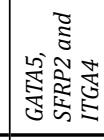 \\
\hline 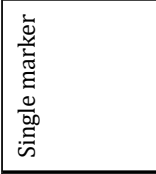 & 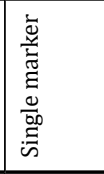 & 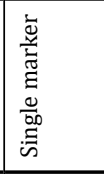 & 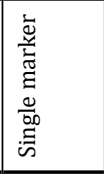 & 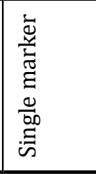 & 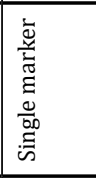 & 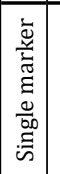 & 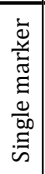 & 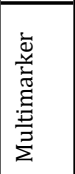 & 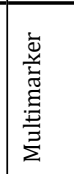 & 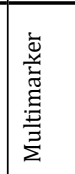 & 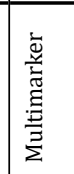 & 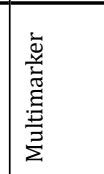 \\
\hline 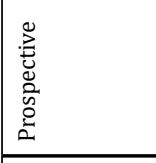 & & 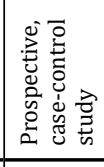 & 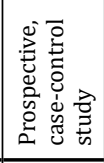 & 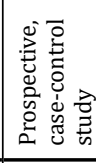 & 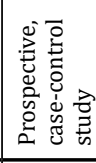 & & & & & & & \\
\hline 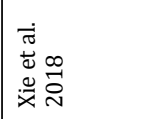 & 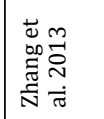 & 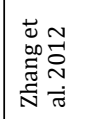 & 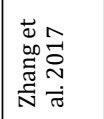 & 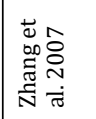 & 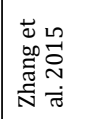 & & & & & & & \\
\hline
\end{tabular}


CHAPTER 2

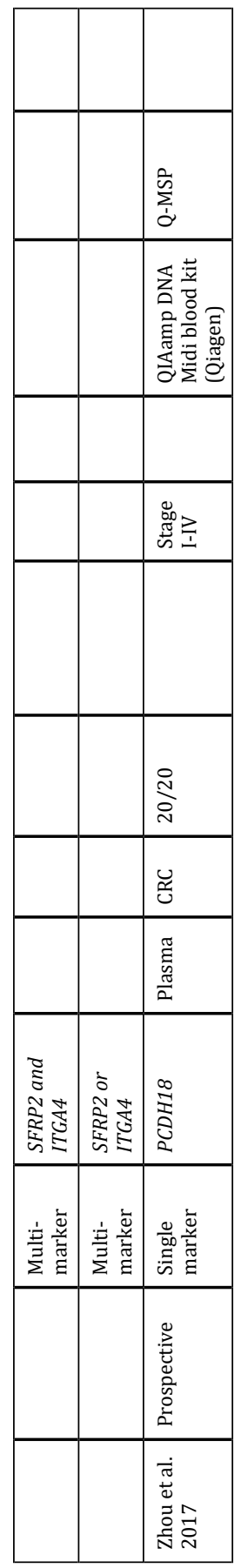


Supplementary Table 5. Reporting quality of included studies

Studies indicated in red potentially have a lower reporting quality. Studies indicated in green potentially have a higher reporting quality.

\begin{tabular}{|c|c|c|c|c|c|c|c|}
\hline & & 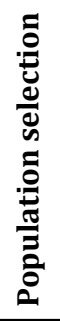 & 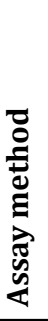 & 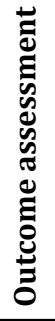 & 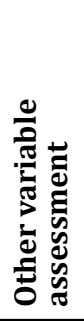 & 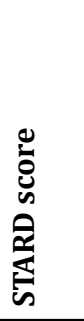 & 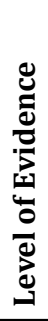 \\
\hline Study & Year & & & & & & \\
\hline Abbaszadegan & 2007 & & & & & 11 & IV \\
\hline Ahlquist & 2012 & & & & & 15.5 & IV \\
\hline Ashoori & 2018 & & & & & 13 & IV \\
\hline Babaei & 2016 & & & & & 9 & IV \\
\hline Baek & 2009 & & & & & 14.5 & III \\
\hline Bartak & 2017 & & & & & 11 & IV \\
\hline Bedin & 2017 & & & & & 11 & IV \\
\hline Belshaw & 2004 & & & & & 8.5 & IV \\
\hline Bosch & 2012 & & & & & 13.5 & IV \\
\hline Cassinotti & 2012 & & & & & 17.5 & IV \\
\hline Chang & 2010 & & & & & 18 & IV \\
\hline Chen & 2017 & & & & & 15.5 & III \\
\hline Chen & 2005 & & & & & 11.5 & IV \\
\hline Church & 2014 & & & & & 18.5 & III \\
\hline
\end{tabular}


CHAPTER 2

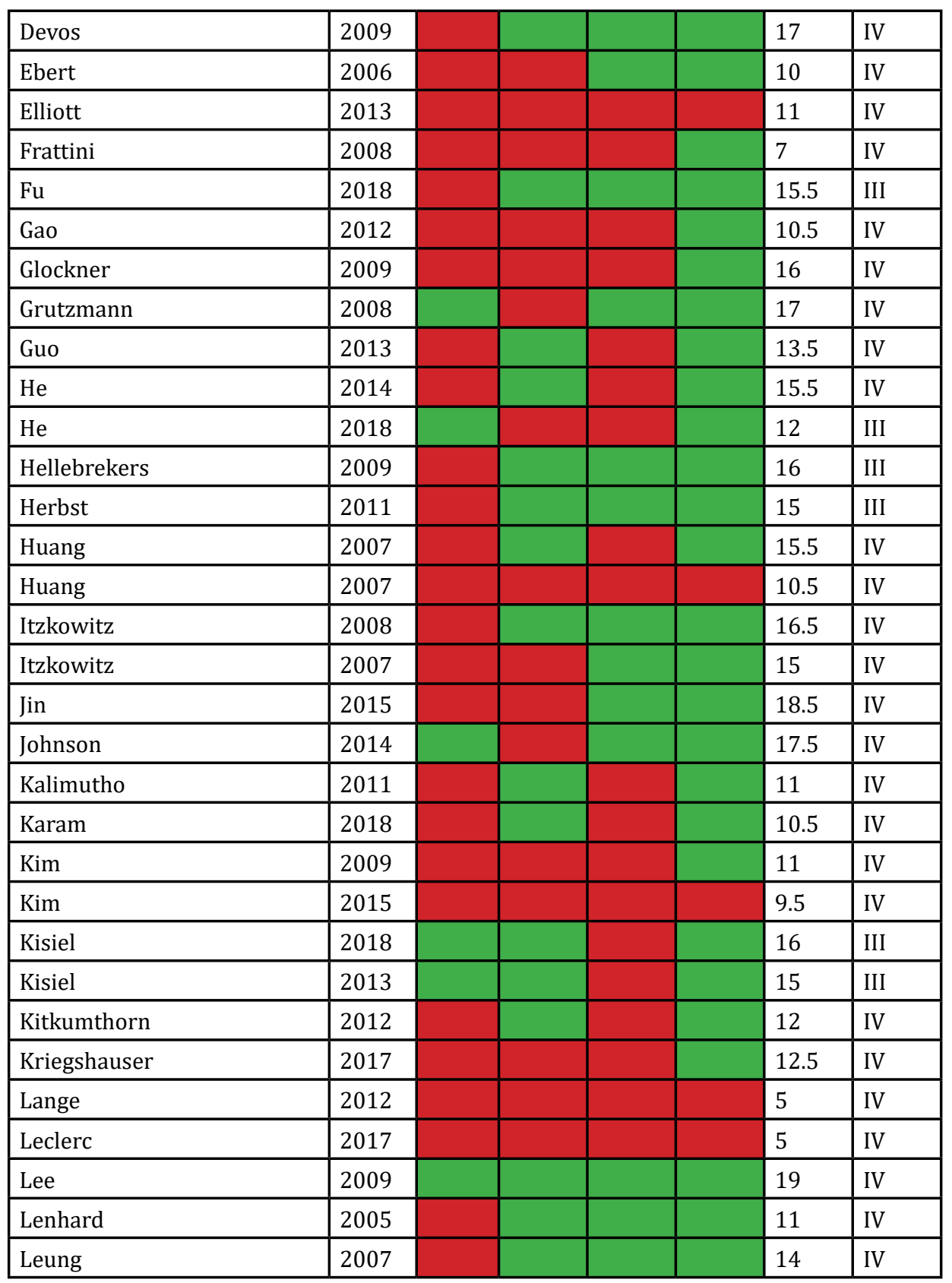




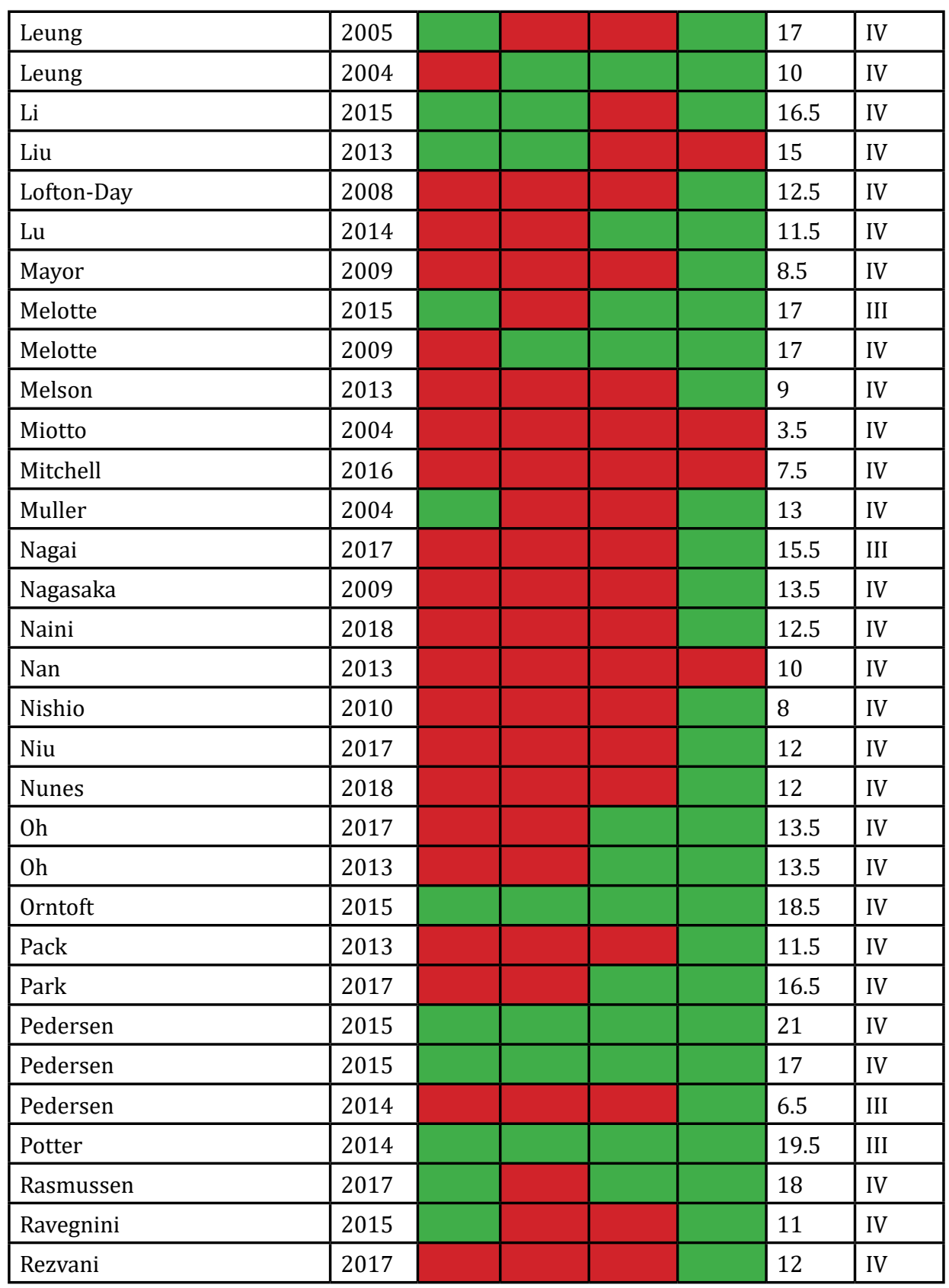


CHAPTER 2

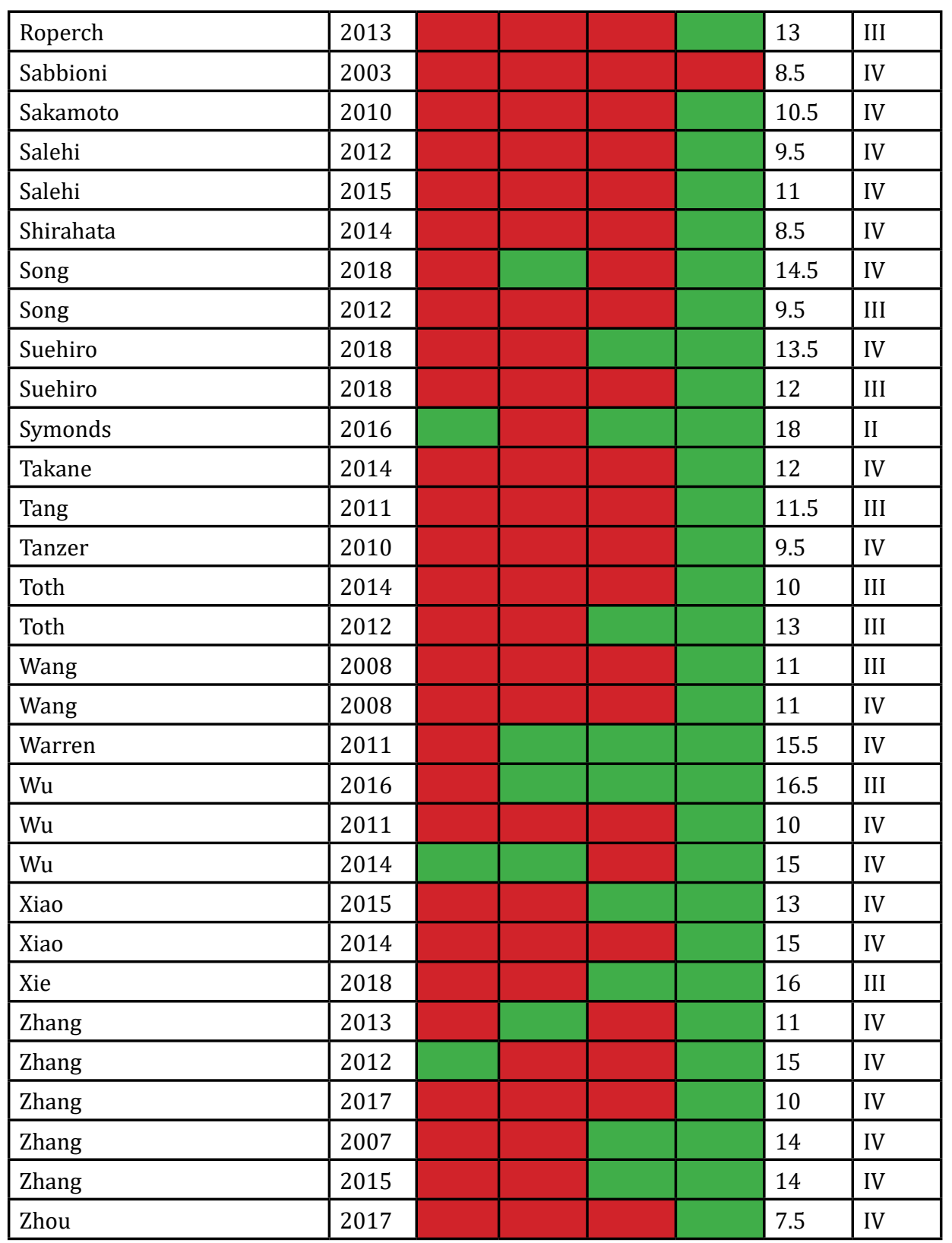


DIAGNOSTIC DNA METHYLATION BIOMARKERS 



\section{CHAPTER 3}

\section{Update on the role of chromoendoscopy in colonoscopic surveillance of patients with Lynch syndrome}

Alouisa J.P. van de Wetering, Roel M.M. Bogie, Assi C.O.G. Cabbolet, Bjorn Winkens, Ad A.M. Masclee, Silvia Sanduleanu 


\section{Abstract}

\section{Objective}

(Virtual) chromoendoscopy (CE) improves the detection of small or flat colorectal polyps, however the evidence in high risk groups, such as patients Lynch syndrome (LS), is low. Our aim was to identify and update the evidence for the recommendations regarding surveillance of Lynch syndrome patients, current underlying evidence for use of (virtual) chromoendoscopy was explored.

\section{Methods}

A systematic literature search in Pubmed, EMBASE and Cochrane library was conducted, for all studies comparing (virtual) CE with white-light endoscopy (WLE) in LS patients. Studies are explained in detail, with special attention to study design, type of (virtual) CE and timing of polypectomy.

\section{Results}

Eight studies (409 patients) were included. Five were nonrandomized back-to-back studies and three were randomized back-to-back studies (1 parallel and 2 cross-over design). In six studies the polyps were directly removed, while in two studies polyps were removed only during the second caecal withdrawal. Five studies researched CE with indigo carmine and three studies investigated virtual CE.

Due to the heterogeneity between studies no statistical analysis can be performed. There is a large variety in study design, timing of polypectomy, different (virtual) CE techniques and the patients which are included.

\section{Conclusion}

Based on current literature, no firm conclusions can be drawn with respect to the additional value of (virtual) CE in the surveillance of patients with LS.

However, training of endoscopists in detection and removal of non-polypoid colorectal neoplasms is crucial, as well as stricter adherence to LS surveillance guidelines in daily clinical practice. For future research, standardisation in study designs is needed. 


\section{Introduction}

Lynch syndrome (LS), the most prevalent hereditary form of colorectal cancer (CRC), is caused by a germline mutation in one of the mismatch repair genes (MLH1, MSH2, MSH6, PMS1 or PMS2) resulting in a 30 to $80 \%$ lifetime risk of developing CRC. ${ }^{1-3}$ The adenoma dwell time, e.g. the time from the development of an adenoma to symptom-detected cancer, is much shorter in LS patients (mean \pm SD: $35.2 \pm 22.3$ months) compared to sporadic CRC where adenoma dwell time is estimated to be at least 10 years. ${ }^{4-6}$

Clinical guidelines recommend 1-2 yearly colonoscopy surveillance intervals for mutation carriers, starting at the age of 20-25 years. ${ }^{7.8}$ Although intensive surveillance has reduced the risk of CRC in LS patients ${ }^{9}$, the cumulative risk of CRC still remains at $6 \%$ after 10 years of follow-up ${ }^{10}$, emphasizing the need for high(er)quality colonoscopy. It has been shown that non-polypoid (flat and depressed) colorectal neoplasms (NP-CRN) are important contributors to interval CRCs in LS patients. NP-CRN are more often found in LS patients compared to average risk controls (OR 3.60, 95\% CI 1.90-6.83), mainly in the proximal colon (OR 6.93, 95\% CI 2.92-16.40). ${ }^{11}$ Detection and endoscopic resection of NP-CRNs is challenging and requires additional endoscopic training. In average-risk populations, polyp miss rate ranges from $2 \%$ to $26 \%$, depending on lesion size. ${ }^{12}$ Polyp miss rate amounts up to $55 \%$ in LS patients ${ }^{13}$, so there is serious concern about the effectiveness of colonoscopy surveillance in this high-risk population. Optimization of endoscopic techniques for detection, diagnosis and resection of neoplasms is a critical step to minimize the interval CRC rate during surveillance for LS. ${ }^{11,14}$

High-definition (HD) white-light colonoscopy (WLE) enhances the detection of CRNs and has become the gold standard in most colonoscopy practices. ${ }^{15}$ In addition, chromoendoscopy (CE) can be used in order to improve detection and characterization of subtle CRNs. There are currently two types of chromoendoscopy: virtual and dye-based chromoendoscopy ${ }^{16}$ (Figure 1). 
Virtual chromoendoscopy includes several modalities such as narrow-band imaging (NBI), autofluorescence endoscopy (AFE), Fuji Intelligent ChromoEndoscopy (FICE) and I-scan. These modalities consist of an incorporated feature to enhance visualization of the surface structures and of the vascular patterns of the mucosal layer ${ }^{17}$, whereas the microvessels are typically enlarged and increased in density in neoplasms. ${ }^{18,19}$ Dye-based chromoendoscopy uses a stain (most commonly indigo carmine (IC)) which has a blue color and is sprayed over the mucosal surface. IC is plant-based and is not absorbed by the mucosa. It forms pools in areas of depth, thereby highlighting subtle pits, depressions and borders of abnormal tissue which facilitates adenoma detection. ${ }^{20}$

Chromoendoscopy with indigo carmine (IC) is described as inexpensive, safe, relatively easy to perform ${ }^{21}$ but is more time consuming. ${ }^{16}$ Virtual CE, on the other hand, is relatively easy to perform and does not add to the costs of an examination ${ }^{22}$, provided this special equipment is available.

Studies comparing (virtual) CE with WLE in average-risk populations show divergent results. In a meta-analysis examining the additional value of narrow band imaging (NBI) over HD-WLE among patients undergoing screening or surveillance colonoscopy, Pasha et al. ${ }^{23}$ found no significant increase in diagnostic yield of colon polyps, adenomas or flat adenomas using NBI. In contrast, a recent Cochrane review concluded that $\mathrm{CE}$ is associated with a significant increase in the detection of colorectal neoplasms compared to WLE in patients undergoing colonoscopy for screening or surveillance. ${ }^{24}$

For high risk patients, e.g. patients with IBD or Lynch syndrome, the European Society of Gastrointestinal Endoscopy (ESGE) recommends the routine use of pancolonic HD-CE in surveillance, however the quality of evidence is low. ${ }^{17}$ 


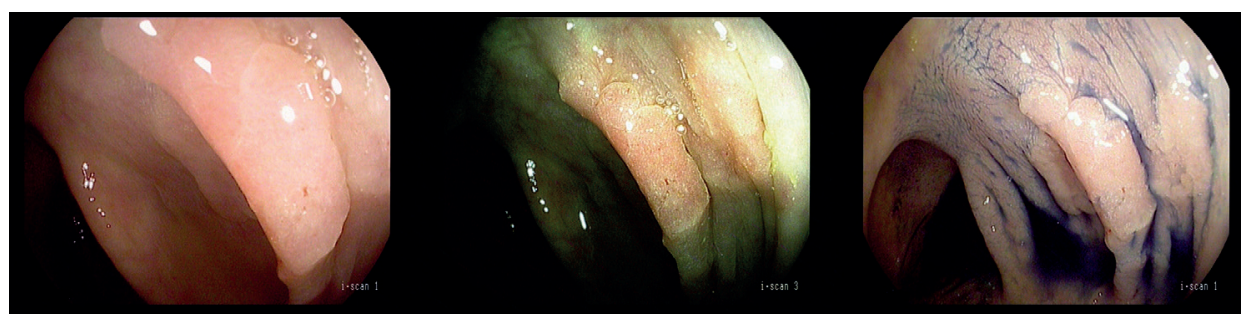

Figure 1. Overview of the different image enhancement techniques, showing a non-granular laterally spreading tumor in the proximal colon of a 44-year old male with Lynch Syndrome. From left to right: White-light endoscopy (high-definition), Virtual chromoendsocopy (I-scan), Dye-based chromoendoscopy with indigo carmine

Where HD-WLE appears to be of additional value in average risk patients ${ }^{23}$, in IBD surveillance CE is preferred over conventional WLE, but also over HD-WLE. ${ }^{16}$ Given the divergence in results regarding high risk patients and the use of CE, WLE or HDWLE and the urgency to optimize quality of surveillance, our aim was to identify and update the evidence for the recommendations as stated above, regarding surveillance of Lynch syndrome patients. In this systematic review, we explored the current underlying evidence for use of (virtual) chromoendoscopy in patients with LS.

\section{Methods}

We conducted a systematic literature search in Pubmed, EMBASE and the Cochrane Library until March 2018 and included articles where white light endoscopy was compared to (virtual) chromoendoscopy, in patients with known or suspected Lynch Syndrome. The following search terms were used: "(C"Colorectal Neoplasms, Hereditary Nonpolyposis"[Mesh]) OR (Lynch syndrome[All fields]) OR (HNPCC[All fields]) OR (HNPCC colorectal[All fields]) OR (hnpcc colorectal cancer) OR (hereditary nonpolyposis colorectal cancer) OR (hereditary non-polyposis colorectal cancer)) AND (("Colonoscopy"[Mesh]) OR (colonoscopy) OR (white light colonoscopy) OR (whitelight colonoscopy) OR (conventional colonoscopy) OR (endoscopy) 
OR ("Endoscopy, Gastrointestinal"[Mesh]) OR (chromoendoscopy) OR (narrow-band imaging) OR (autofluorescence endoscopy) OR (FICE) OR (I-scan))".

Two study investigators (A.W. and A.C.) screened all abstracts independently. Review articles, case reports and studies on other topics on LS patients were excluded. The full text of the remaining studies was examined. In case of discrepancy, data were reviewed by the study coordinator (S.S.) and discussed to reach agreement. The Preferred Reporting Items for Systematic Reviews (PRISMA) methodology has been employed for conducting and reporting a systematic review. ${ }^{25}$ Quality assessment was performed using the Quality Assessment of Diagnostic Accuracy Studies (QUADAS) tool (Supplementary Table 1). ${ }^{26}$ We also used the Grading of Recommendations, Assessment, Development and Evaluation (GRADE) approach for grading the quality of evidence. ${ }^{27}$

\section{Study designs}

Different study designs were used to compare the diagnostic yield of (virtual) CE and WLE in colonoscopic surveillance for LS. In a nonrandomized back-to-back study, the same patient group underwent colonoscopy twice. After reaching the caecum, the first image modality was used (first caecal withdrawal). Then, the caecum was reached again, and the second image modality was applied (second caecal withdrawal). In a randomized trial, patients were randomized for the order of different techniques, which were performed using a parallel or cross-over design. 


\section{Results}

We retrieved 981 articles. Of these, 8 studies (409 patients) were included in this review. The search strategy is shown in Figure 2. Five studies used a nonrandomized back-to-back design ${ }^{28-32}$, one used a randomized parallel design ${ }^{13}$ and two a randomized cross-over design. ${ }^{22,33}$ Figure 3 summarizes the study designs.

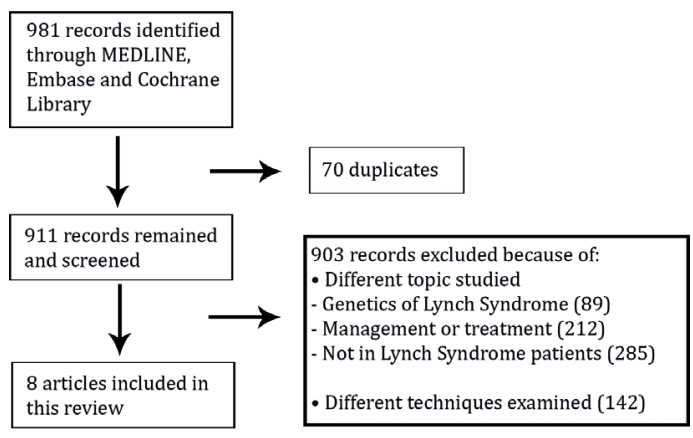

Figure 2. Overview of study selection for the systematic review comparing (virtual) chromoendoscopy with white-light endoscopy (conventional and high-definition) in patients with Lynch syndrome

In six studies the polyps were removed during first and second caecal withdrawal ${ }^{13,22,28-31}$, while in the other two studies all polyps detected during the first caecal withdrawal were documented and removed only during the second caecal withdrawal with CE. ${ }^{32,33}$ In 2 studies a different endoscopist performed the second examination and the endoscopist was blinded to the findings during the first procedure. ${ }^{31,33}$ An overview of the study characteristics and the levels of evidence using the GRADE approach is shown in Table 1 and the results are shown in Table 2. 
Figure 3. Overview of study designs of the studies included in this review.

Nonrandomized back-to-back design
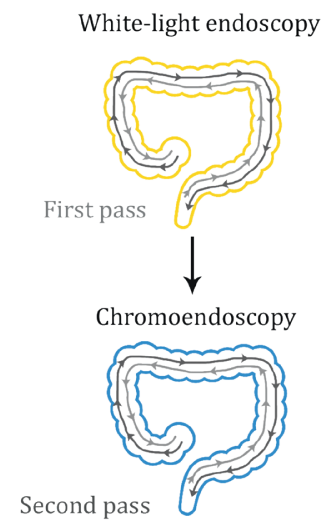

\section{Randomized parallel back-to-back design}
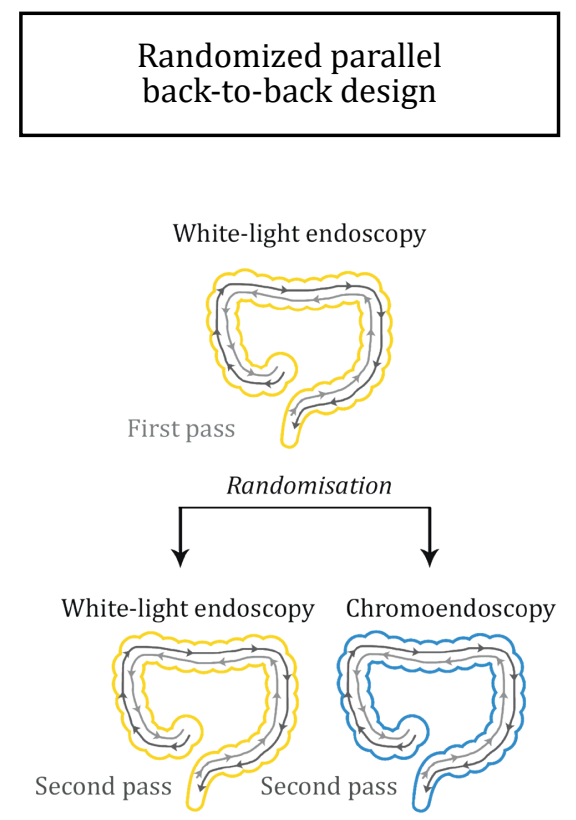

White-light endoscopy

\section{Nonrandomized parallel back-to-back design}
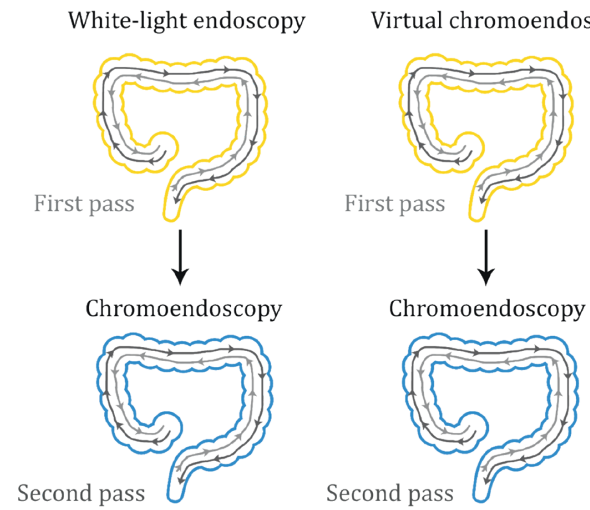

Chromoendoscopy

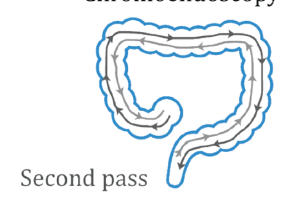

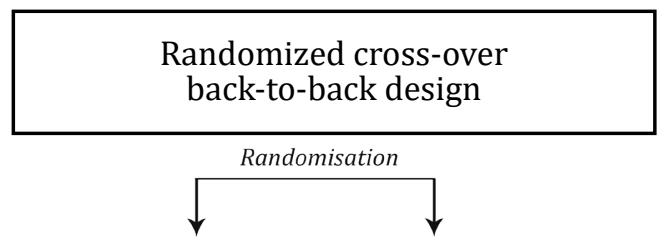
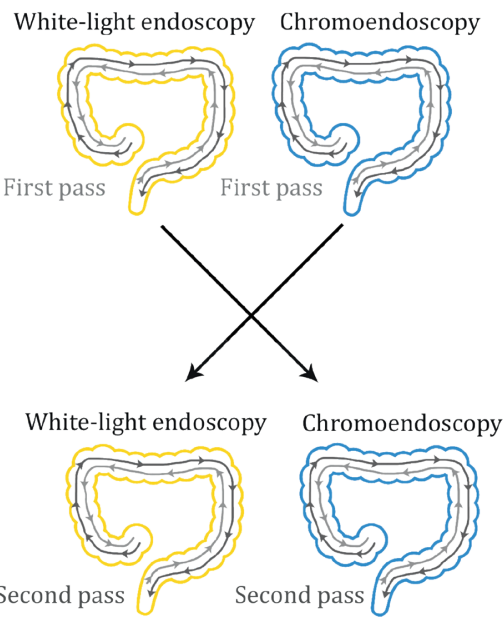
Table 1. Characteristics of the studies of the detection of polyps and (flat) adenomas with white light endoscopy (WLE) and (virtual) chromoendoscopy (CE) in patients with Lynch syndrome (LS).

\begin{tabular}{|c|c|c|c|c|c|}
\hline Author & $\begin{array}{l}\text { Year, } \\
\text { Country }\end{array}$ & Study design & Setting & Participants & GRADE \\
\hline $\begin{array}{l}\text { Hurlstone } \\
\text { et al. }{ }^{28}\end{array}$ & $\begin{array}{l}2005, \\
\text { United } \\
\text { Kingdom }\end{array}$ & $\begin{array}{l}\text { Nonrandomized, } \\
\text { back-to-back } \\
\text { study }\end{array}$ & $\begin{array}{l}\text { - Direct polyp removal } \\
\text { - Single endoscopist } \\
\text { - Single center }\end{array}$ & $\begin{array}{l}25 \text { patients meeting the Amster- } \\
\text { dam criteria ( } 21 \text { MMR mutation } \\
\text { carriers; MLH1 or MSH2) }\end{array}$ & $\begin{array}{l}\text { Moderate } \\
\text { quality }\end{array}$ \\
\hline $\begin{array}{l}\text { Lecomte } \\
\text { et al. }{ }^{29}\end{array}$ & $\begin{array}{l}\text { 2005, } \\
\text { France }\end{array}$ & $\begin{array}{l}\text { Nonrandomized, } \\
\text { back-to-back } \\
\text { study }\end{array}$ & $\begin{array}{l}\text { - Direct polyp removal } \\
\text { - Single endoscopist } \\
\text { - Single center }\end{array}$ & $\begin{array}{l}36 \text { patients (18 patients meeting } \\
\text { Amsterdam I or II criteria / } 18 \\
\text { MMR mutation carriers (MSH2: } \\
12 \text { patients, MLH1: } 6 \text { patients)) }\end{array}$ & $\begin{array}{l}\text { Moderate } \\
\text { quality }\end{array}$ \\
\hline $\begin{array}{l}\text { Stoffel } \\
\text { et al. }{ }^{13}\end{array}$ & $\begin{array}{l}2008, \\
\text { USA }\end{array}$ & $\begin{array}{l}\text { Randomized, } \\
\text { parallel, back-to- } \\
\text { back study } \\
\text { (randomization } \\
\text { after first proce- } \\
\text { dure) } \\
\end{array}$ & $\begin{array}{l}\text { - Direct polyp removal } \\
\text { - } 7 \text { endoscopists } \\
\text { - Multicenter }\end{array}$ & $\begin{array}{l}\text { Total } 54 \text { patients } \\
\text { Subset used: } 28 \text { patients meet- } \\
\text { ing Amsterdam criteria } \\
\text { (23 MMR mutation carriers; } \\
\text { MLH1: } 6 \text { patients, MSH2:17 } \\
\text { patients) }\end{array}$ & $\begin{array}{l}\text { Moderate } \\
\text { quality }\end{array}$ \\
\hline $\begin{array}{l}\text { East } \\
\text { et al. }{ }^{30}\end{array}$ & $\begin{array}{l}2008, \\
\text { United } \\
\text { Kingdom }\end{array}$ & $\begin{array}{l}\text { Nonrandomized, } \\
\text { back-to-back } \\
\text { study }\end{array}$ & $\begin{array}{l}\text { - Direct polyp removal } \\
\text { - } 3 \text { endoscopists } \\
\text { - Single center }\end{array}$ & $\begin{array}{l}62 \text { patients meeting Amsterdam } \\
\text { II criteria (8 MMR mutation car- } \\
\text { riers; MLH1: } 3 \text { patients, MSH2: } 4 \\
\text { patients, MSH6: } 1 \text { patient) }\end{array}$ & $\begin{array}{l}\text { Moderate } \\
\text { quality }\end{array}$ \\
\hline $\begin{array}{l}\text { Hüneburg } \\
\text { et al. }{ }^{32}\end{array}$ & $\begin{array}{l}2009, \\
\text { Germany }\end{array}$ & $\begin{array}{l}\text { Nonrandomized, } \\
\text { (parallel) back- } \\
\text { to-back study }\end{array}$ & $\begin{array}{l}\text { - Polyp removal } \\
\text { during second pro- } \\
\text { cedure (documented } \\
\text { by independent } \\
\text { observer during first } \\
\text { procedure) } \\
\text { - } 2 \text { endoscopists } \\
\text { - Single center }\end{array}$ & $\begin{array}{l}\text { Total } 114 \text { patients } \\
\text { Subset used: } 47 \text { patients meet- } \\
\text { ing Amsterdam II criteria ( } 42 \\
\text { MMR mutation carriers) }\end{array}$ & $\begin{array}{l}\text { Moderate } \\
\text { quality }\end{array}$ \\
\hline $\begin{array}{l}\text { Ramsoekh } \\
\text { et al. }\end{array}$ & $\begin{array}{l}2010, \\
\text { The } \\
\text { Nether- } \\
\text { lands }\end{array}$ & $\begin{array}{l}\text { Randomized, } \\
\text { back-to-back, } \\
\text { cross over study }\end{array}$ & $\begin{array}{l}\text { - Polyp removal } \\
\text { during second pro- } \\
\text { cedure (lesions were } \\
\text { photographed) } \\
\text { - } 2 \text { endoscopists } \\
\text { during one session } \\
\text { (blinded for the previ- } \\
\text { ous findings) } \\
\text { - Single center }\end{array}$ & $\begin{array}{l}75 \text { patients: } \\
\text { - } 41 \text { familial risk of CRC } \\
-34 \text { patients with LS } \\
\text { (29 MMR mutation carriers and } \\
5 \text { patients meeting Amsterdam } \\
\text { criteria) }\end{array}$ & $\begin{array}{l}\text { Low / } \\
\text { Moderate } \\
\text { quality }\end{array}$ \\
\hline $\begin{array}{l}\text { Rahmi } \\
\text { et al. }{ }^{31}\end{array}$ & $\begin{array}{l}\text { 2015, } \\
\text { France }\end{array}$ & $\begin{array}{l}\text { Nonrandomized, } \\
\text { back-to-back } \\
\text { study }\end{array}$ & $\begin{array}{l}\text { - Direct polyp removal } \\
\text { - } 2 \text { endoscopists } \\
\text { (blinded for the } \\
\text { results of the first } \\
\text { procedure and rand- } \\
\text { omized) } \\
\text { - Multicenter }\end{array}$ & $\begin{array}{l}78 \text { MMR mutation carriers } \\
\text { (MLH1: } 26 \text { patients, MSH2: } 43 \\
\text { patients, MSH6: } 9 \text { patients) }\end{array}$ & $\begin{array}{l}\text { Moderate } \\
\text { /High } \\
\text { quality }\end{array}$ \\
\hline $\begin{array}{l}\text { Bisschops } \\
\text { et al. }{ }^{22}\end{array}$ & $\begin{array}{l}\text { 2017, } \\
\text { Belgium }\end{array}$ & $\begin{array}{l}\text { Randomized, } \\
\text { back-to-back, } \\
\text { cross over study }\end{array}$ & $\begin{array}{l}\text { - Direct polyp removal } \\
\text { - } 2 \text { endoscopists } \\
\text { - Single center }\end{array}$ & $\begin{array}{l}61 \text { patients meeting Amsterdam } \\
\text { II criteria (39 MMR mutation } \\
\text { carriers (MSH6: } 12 \text { patients, } \\
\text { MSH2: } 20 \text { patients, MLH1: } 7 \\
\text { patients) }\end{array}$ & $\begin{array}{l}\text { High } \\
\text { quality }\end{array}$ \\
\hline \multicolumn{6}{|c|}{$\begin{array}{l}\text { MMR: mismatch repair, IC: indigo carmine, used for dye spray chromoendoscopy, NBI: narrow band imaging, AFE: } \\
\text { autofluorescence endoscopy, HD-WLE: high-definition white light endoscopy, GRADE: Grading of Recommendations, } \\
\text { Assessment, Development and Evaluation }\end{array}$} \\
\hline
\end{tabular}




\section{CHAPTER 3}

Table 2. Results of the different studies of the detection of polyps and (flat) adenomas with white light endoscopy (WLE) and (virtual) chromoendoscopy (CE) in patients with Lynch syndrome (LS).

\begin{tabular}{|c|c|c|c|c|c|c|c|c|}
\hline \multirow[t]{2}{*}{ Author } & \multirow[t]{2}{*}{$\begin{array}{c}\text { Number of } \\
\text { patients }\end{array}$} & \multirow[t]{2}{*}{ Methods compared } & \multicolumn{2}{|c|}{$\begin{array}{l}\text { Patients with } \\
\geq 1 \text { adenoma }\end{array}$} & \multicolumn{2}{|c|}{$\begin{array}{l}\text { Number of } \\
\text { adenomas } \\
\text { detected }\end{array}$} & \multicolumn{2}{|c|}{$\begin{array}{c}\text { Number } \\
\text { of flat } \\
\text { adenomas } \\
\text { detected }\end{array}$} \\
\hline & & & WLE & CE & WLE & CE & WLE & CE \\
\hline Hurlstone et al. ${ }^{28}$ & 25 & $\begin{array}{l}\text { Conventional WLE vs } \\
\text { pancolonic CE (IC) }\end{array}$ & 7 & 17 & 11 & 32 & 4 & 35 \\
\hline Lecomte et al. ${ }^{29}$ & 33 & $\begin{array}{l}\text { Conventional WLE vs } \\
\text { CE (IC) in proximal } \\
\text { colon }\end{array}$ & 3 & 8 & 7 & 11 & 2 & 8 \\
\hline Stoffel et al. ${ }^{13}$ & 54 & $\begin{array}{l}\text { 1. Conventional WLE } \\
\text { vs pan-colonic CE } \\
\text { (IC) }(n=28) \\
\text { 2. WLE vs } \\
\text { intensive inspection }\end{array}$ & $8 / 54$ & $3 / 28$ & 10 & 5 & 4 & 3 \\
\hline East et al. ${ }^{30}$ & 62 & $\begin{array}{l}\text { Conventional WLE vs } \\
\text { virtual CE using NBI }\end{array}$ & 17 & 26 & 25 & 46 & 3 & 9 \\
\hline Hüneburg et al. ${ }^{32}$ & 109 & $\begin{array}{l}\text { 1. Conventional WLE } \\
\text { vs pancolonic CE } \\
\text { (IC) }(\mathrm{n}=47) \\
\\
\text { 2. Virtual CE using } \\
\text { NBI vs pancolonic } \\
\text { CE (IC) }\end{array}$ & $7 / 109$ & $9 / 47$ & 7 & 13 & 4 & 8 \\
\hline Ramsoekh et al. ${ }^{33}$ & 75 & $\begin{array}{l}\text { 1. Conventional WLE } \\
\text { vs virtual CE using } \\
\text { AFE } \\
\text { 2. Virtual CE using } \\
\text { AFE vs WLE }\end{array}$ & 28 & 37 & 8 & 30 & 1 & 6 \\
\hline Rahmi et al. ${ }^{31}$ & 78 & $\begin{array}{l}\text { Conventional WLE vs } \\
\text { pancolonic CE (IC) }\end{array}$ & 18 & 24 & 26 & 29 & 8 & 14 \\
\hline Bisschops et al. ${ }^{22}$ & 61 & $\begin{array}{l}\text { 1. HD-WLE vs } \\
\text { virtual CE using } \\
\text { I-scan } \\
\text { 2. Virtual CE using } \\
\text { I-scan vs HD-WLE }\end{array}$ & 8 & 14 & 7 & 23 & - & - \\
\hline
\end{tabular}


Due to the heterogeneity between the studies a meta-analysis could not be performed. There are differences in study design (nonrandomized versus randomized; parallel and cross-over), but also regarding timing of polypectomy (directly after detection (first and second withdrawal) or described and remove during the second withdrawal) and different (virtual) CE techniques are compared to conventional or high-definition WLE. Furthermore, mismatch repair (MMR) gene mutation carriers were included, but also patients with suspected LS (according to the Amsterdam I or II criteria) and patients with familiar risk of CRC, all with relatively low number of inclusions.

The results of the individual studies in this systematic review are described per study design where the differences in study characteristics are shown along with the outcomes. An overview of the results is shown in Table 2.

\section{Non-randomized back-to-back design - Chromoendoscopy with indigo carmine}

Hurlstone et al. ${ }^{28}$ included 25 LS patients, which underwent first conventional WLE and thereafter CE with indigo carmine (IC), with polyp removal during first and second caecal withdrawal. Using $\mathrm{CE}$, more patients with $\geq 1$ adenoma were found compared to WLE (7 vs. 17 patients). Fifty-two additional lesions were found using $\mathrm{CE}$, as well as significantly more adenomas $(p=0.001)$ and flat adenomas $(p=0.004)$.

Lecomte et al. ${ }^{29}$ used a similar design, where among 33 patients, 5 additional patients with $\geq 1$ adenoma were found. In total 45 additional lesions were conducted using CE, from which 11 were adenomas (8 flat).

In the study by Rahmi et al. ${ }^{31} 78$ MMR carriers underwent conventional WLE, then CE was performed by a second endoscopist, blinded for previous findings. The number of patients with $\geq 1$ adenoma increased from 18 during WLE to 32 with CE $(p<0.001)$. Overall during WLE 98 lesions were detected and 188 additional lesions were found during CE. With use of CE, more (flat) adenomas were found (29 adenomas using CE vs. 26 adenomas with WLE; 14 flat vs. 8 flat respectively). 
Hüneberg et al. ${ }^{32}$ used a parallel design comparing CE to WLE and CE to NBI, where during the first procedure polyps were documented by an independent observer, and removed during the second procedure. 47 patients were included in the WLE vs. CE study arm, WLE detected 7 patients with $\geq 1$ adenoma and CE 9 patients with $\geq 1$ adenoma. Significantly more lesions $(p=0.006)$ and flat adenomas were found using CE (7 adenomas, 4 flat vs. 13 adenomas, 8 flat, $p=0.004$ ).

\section{Non-randomized back-to-back design - Virtual chromoendoscopy}

East et al. ${ }^{30}$ compared conventional WLE to narrow band imaging (NBI) in 62 patients with known or suspected LS where the number of patients with $\geq 1$ adenoma rose from 17 after WLE, to 26 after NBI ( $p=0.004)$. The total number of adenomas was 25 (3 flat) before, and 46 (9 flat) after NBI ( $p<0.001)$.

\section{Randomized back-to-back design - Chromoendoscopy with indigo carmine}

Stoffel et al. ${ }^{13}$ used an interesting approach to compare WLE to CE and intensive inspection (a thorough examination of the colon without indigo carmine dye, where endoscopists should spend at least 20 minutes visualizing the colonic mucosa during withdrawal). After complete WLE examination, patients were randomized between $\mathrm{CE}$ and intensive inspection. No significant difference in adenoma detection was found between $\mathrm{CE}$ and intensive inspection ( $p=0.27)$ but CE detected more polyps ( $p=0.004)$. Another important conclusion is that CE was associated with more normal tissue biopsies and longer procedure time compared to intensive inspection.

\section{Randomized back-to-back design - Virtual chromoendoscopy}

Autofluorescence endoscopy (AFE) and conventional WLE were compared in the study by Ramsoekh et al. ${ }^{33}$, where polyps were removed during the second procedure. Of note, this study included LS patients $(n=34)$ and patients with familial CRC risk $(\mathrm{n}=41)$. At least one adenoma was detected in 41 patients, where WLE identified 28 patients and AFE identifies 37 patients. Regarding adenoma detection, 22 additional adenomas (5 flat) were detected during AFE. 
Bisschops et al. ${ }^{22}$ performed a study to compare high definition (HD-)WLE and I-scan, using a cross over design where polyps were directly removed. I-scan detected more patients with $\geq 1$ adenoma compared to HD-WLE (14 vs. 8), and showed 23 additional adenomas were found using I-scan. Furthermore, this group researched the effect of the inspection time, showing no difference in inspection time between the different imaging techniques.

\section{Discussion}

It is concluded that from this systematic review no firm conclusions can be drawn with respect to the additional value of (virtual) chromoendoscopy in the surveillance of patients with Lynch Syndrome. However, it is of great importance to acknowledge various relevant factors and take these factors into account.

First of all, a variety of different study designs were used to compare (virtual) CE and WLE in colonoscopic surveillance for LS with a wide variation in methodology across the studies. In a randomized parallel design, the expected number of polyps will be approximately the same in both study arms and the results will be unbiased. However, according to van den Broek et al. ${ }^{34}$ there is variation between trials using this design due to a difference in polyps at baseline resulting in random noise. To reduce this noise, a back-to-back design with fixed order can be used. However, this is not an ideal design to investigate whether the novel technique, CE in this case, should be added to existing technique.

Furthermore, a back-to-back design is known to have limitations. It can be argued that in a back-to-back design the second inspection ( $>6$ minutes) itself may result in a better lesion detection. ${ }^{35}$ In line with this, in a randomized study, Stoffel et al. ${ }^{13}$ compared CE vs. WLE with intensive inspection ( $>20$ minutes), after a first examination with WLE. No significant difference was found between the two techniques. In the study by Bisschops et al. ${ }^{22}$ the effect of a second inspection was investigated, where any second inspection appears shorter than the first 
inspection, regardless whether HD-WLE or virtual CE was used. This indicates that the increased detection rate during second exam is likely due to the use of advanced imaging techniques (instead of longer inspection time).

Secondly, timing of polypectomy needs to be taken into consideration. This differs between studies (e.g. during the first and second caecal withdrawal vs. during second caecal withdrawal only).

With direct removal of polyps, only missed polyps can be picked up during the second examination. This might be preferable to investigate whether a technique can detect additional polyps. If polyps are not directly removed, but only during the second caecal withdrawal, the two techniques for each polyp can be better compared, within a patient. Another advantage of the latter is it states which types of polyps are more difficult to detect comparing the two techniques by evaluation of the characteristics of missed polyps. ${ }^{34}$

Thirdly, different image modalities are used. High-definition (HD) white-light colonoscopy (WLE) enhances the detection of CRNs and has become the gold standard in most colonoscopy practices. ${ }^{15}$ It should be acknowledged that in 7 of the 8 studies we included in our analysis have used conventional WLE. Dye-based and virtual chromoendoscopy (CE) improve detection and characterization of subtle CRNs. A recent Cochrane review has provided evidence that both dye-based and virtual CE enhance detection of neoplasia in patients undergoing screening or surveillance colonoscopy. However, in that analyses LS patients have been excluded. ${ }^{24}$

Fourthly, it should be taken into account that the studies focussing on different colonoscopy techniques in LS patients have included different patient groups: not only variations in types of gene mutation carriers, but also patients fulfilling the Amsterdam I or II criteria and even familial CRC have been included. It has previously been shown that MLH-1 and MSH-2 gene mutation carriers have a higher risk of developing CRC compared to MSH-6 and PMS-2 carriers. ${ }^{36}$ 
To optimize the yield of endoscopic surveillance in LS patients, in order to reduce the risk of CRC in LS patients, a number of essential other factors need to be taken into account.

First, it has been shown that training in endoscopic detection and resection of NP-CRNs is critical for optimizing the quality and effectiveness of colonoscopic surveillance in LS patients. A case-control study by Rondagh et al. showed that in LS patients, NP-CRNs are found nearly four times more often compared to average-risk patients. ${ }^{11}$ In that study, all endoscopists (faculty and trainees) were trained in the detection of NP-CRNs using a systematic training curriculum consisting of lectures, video-training and individual feedback. ${ }^{11}$ Obviously, endoscopists who are proficient in the detection of NP-CRNs have significantly higher (flat) adenoma detection rates compared to untrained endoscopists. ${ }^{37}$ For the integration of (virtual) CE in current practice, the use of HD-equipment, standard materials and a standard operating procedure is considered to be a prerequisite. ${ }^{38,39}$

Second, another important factor in reducing the risk of CRC in LS patients is compliance with surveillance intervals. A 2015-study from the UK found that compliance to a 2-yearly surveillance in $227 \mathrm{LS}$ patients was only $68 \%{ }^{40}$ In 2007 the surveillance strategy in LS patients in the Netherlands has been evaluated using a nationwide survey, where $86 \%$ of physicians used standard colonoscopy without any additional imaging techniques. Fifty percent of the physicians expected CE to be incorporated into surveillance practice in LS patients in the near future. ${ }^{41}$

Based on our analysis recommendations can be made for future studies on the added value of (virtual) CE over HD-WLE. A randomized cross-over trial would be an efficient study design for comparing two techniques, where each patient can serve as his/her own control (Figure 4). When using a second endoscopist, blinded for previous findings, the 'second look effect' will be limited. Another advantage would be that evaluation of the characteristics of the missed polyps can be performed. Ideally, a large number of patients should be included so a stratification by type of MMR mutation can be made. 
Certain limitations with respect to this systematic review should be considered. Especially the heterogeneity in study design, timing of polypectomy, different types of image modalities, the criteria for selection of the study population and the small number of studies and patients are of relevance. Due to these factors, no statistical analysis could be performed.

It is concluded that from this systematic review no firm conclusions can be drawn with respect to the additional value of (virtual) chromoendoscopy in the surveillance of patients with Lynch Syndrome. However, certain factors should be acknowledged. First, training of endoscopists in endoscopic detection and removal of NP-CRNs is crucial. Second, standardisation in design of studies on yield of surveillance colonoscopy is needed, as well as stricter adherence to LS surveillance guidelines in daily clinical practice. Finally, the cumulative risk for CRC in LS remains substantial and allows an intensified search for better strategies. 
Figure 4. Proposed study design for future studies investigating white light endoscopy (WLE) versus (virtual) chromoendoscopy in patients with Lynch Syndrome: Randomized cross-over back-to-back study design.

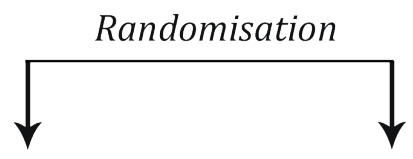

White-light endoscopy Chromoendoscopy

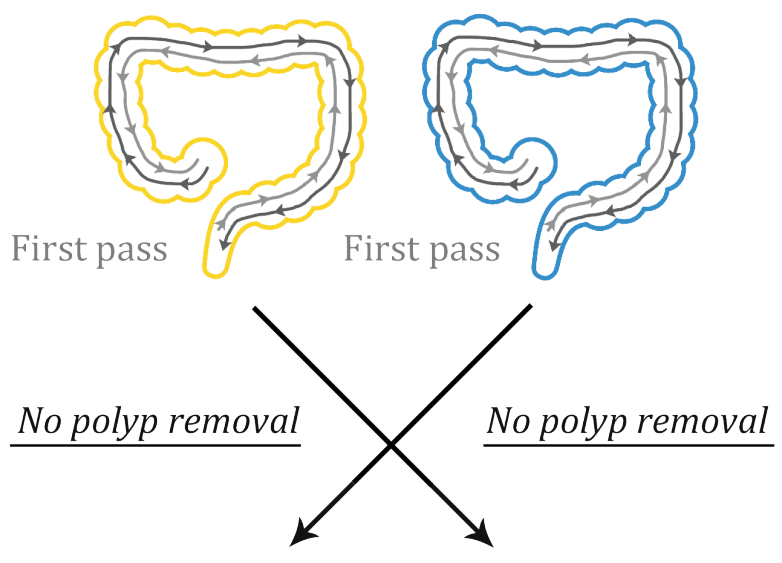

White-light endoscopy Chromoendoscopy

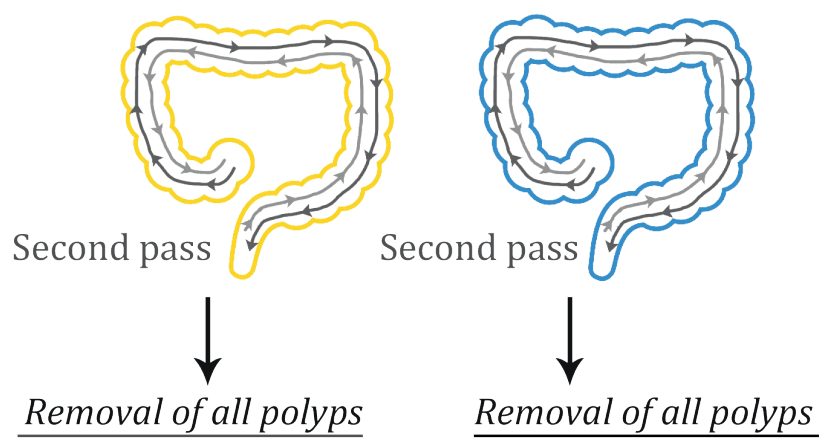




\section{References}

1. Lynch HT, de la Chapelle A. Hereditary colorectal cancer. N Engl J Med 2003;348(10):919-32.

2. Vasen HF. Review article: The Lynch syndrome (hereditary nonpolyposis colorectal cancer). Aliment Pharmacol Ther 2007;26 Suppl 2:113-26.

3. Stoffel EM, et al. Prevalence and predictors of appropriate colorectal cancer surveillance in Lynch syndrome. Am J Gastroenterol 2010;105(8):1851-60.

4. Edelstein DL, et al. Rapid development of colorectal neoplasia in patients with Lynch syndrome. Clin Gastroenterol Hepatol 2011;9(4):340-3.

5. Jenkins MA, et al. Short-term risk of colorectal cancer in individuals with lynch syndrome: a meta-analysis. J Clin Oncol 2015;33(4):326-31

6. Kuntz KM, et al. A systematic comparison of microsimulation models of colorectal cancer: the role of assumptions about adenoma progression. Med Decis Making 2011;31(4):530-9.

7. Vasen HF, et al. Revised guidelines for the clinical management of Lynch syndrome (HNPCC) recommendations by a group of European experts. Gut 2013;62(6):812-23
8. Giardiello FM, et al. Guidelines on genetic evaluation and management of Lynch syndrome: a consensus statement by the US Multi-society Task Force on colorectal cancer. Am J Gastroenterol 2014;109(8):1159-79.

9. de Vos tot Nederveen Cappel WH, et al. Surveillance for hereditary nonpolyposis colorectal cancer: a long-term study on 114 families. Dis Colon Rectum 2002;45(12):158894.

10. Vasen HF, et al. One to 2-year surveillance intervals reduce risk of colorectal cancer in families with Lynch syndrome. Gastroenterology 2010;138(7):2300-6.

11. Rondagh EJ, et al. Nonpolypoid colorectal neoplasms: a challenge in endoscopic surveillance of patients with Lynch syndrome. Endoscopy 2013;45(4):257-64.

12. van Rijn JC, et al. Polyp miss rate determined by tandem colonoscopy: a systematic review. Am J Gastroenterol 2006;101(2):343-50.

13. Stoffel EM, et al. Missed adenomas during colonoscopic surveillance in individuals with Lynch Syndrome (hereditary nonpolyposis colorectal cancer). Cancer Prev Res (Phila) 2008;1(6):470-5.

14. Haanstra JF, et al. Quality colonoscopy and risk of interval cancer in Lynch syndrome. Int J Colorectal Dis 2013;28(12):1643-9. 
15. Rex DK, Helbig CC. High Yields of Small and Flat Adenomas With High-Definition Colonoscopes Using Either White Light or Narrow Band Imaging. Gastroenterology 2007;133(1):42-7.

16. Laine L, et al. SCENIC international consensus statement on surveillance and management of dysplasia in inflammatory bowel disease. Gastrointest Endosc 2015;81(3):489-501 e26.

17. Kaminski MF, et al. Advanced imaging for detection and differentiation of colorectal neoplasia: European Society of Gastrointestinal Endoscopy (ESGE) Guideline. Endoscopy 2014;46(5):435-49.

18. Haanstra JF, et al. Role of new endoscopic techniques in Lynch syndrome. Fam Cancer 2013;12(2):26772 .

19. Hazewinkel Y, Dekker E. Colonoscopy: basic principles and novel techniques. Nat Rev Gastroenterol Hepatol 2011;8(10):554-64.

20. McGill S, et al. Image-enhanced endoscopy in practice. Can J Gastroenterol 2009;23(11):741-6.

21. ASGE Committee, et al. Chromoendoscopy. Gastrointest Endosc 2007;66(4):639-49.
22. Bisschops $\mathrm{R}$, et al. Virtual chromoendoscopy (I-SCAN) detects more polyps in patients with Lynch syndrome: a randomized controlled crossover trial. Endoscopy 2017;49(4):342-50.

23. Pasha SF, et al. Comparison of the yield and miss rate of narrow band imaging and white light endoscopy in patients undergoing screening or surveillance colonoscopy: a meta-analysis. Am J Gastroenterol 2012;107(3):363-70; quiz 71.

24. Brown Steven R, et al. Chromoscopy versus conventional endoscopy for the detection of polyps in the colon and rectum. Cochrane Database of Systematic Reviews. 2016 (4). Available from: http://onlinelibrary. wiley.com/doi/10.1002/14651858. CD006439.pub4/abstract

25. Moher D, et al. Preferred reporting items for systematic reviews and meta-analyses: the PRISMA statement. Int J Surg 2010;8(5):336-41.

26. Whiting PF, et al. Evaluation of QUADAS, a tool for the quality assessment of diagnostic accuracy studies. BMC Med Res Methodol 2006;6:9.

27. Guyatt GH, et al. GRADE: an emerging consensus on rating quality of evidence and strength of recommendations. BMJ 2008;336(7650):924-6. 
28. Hurlstone DP, et al. The role of high-magnification-chromoscopic colonoscopy in hereditary nonpolyposis colorectal cancer screening: a prospective "backto-back" endoscopic study. Am J Gastroenterol 2005;100(10):216773.

29. Lecomte T, et al. Chromoendoscopic colonoscopy for detecting preneoplastic lesions in hereditary nonpolyposis colorectal cancer syndrome. Clin Gastroenterol Hepatol 2005;3(9):897-902.

30. East JE, et al. Narrow band imaging for colonoscopic surveillance in hereditary non-polyposis colorectal cancer. Gut 2008;57(1):65-70.

31. Rahmi G, et al. Impact of chromoscopy on adenoma detection in patients with Lynch syndrome: a prospective, multicenter, blinded, tandem colonoscopy study. Am J Gastroenterol 2015;110(2):288-98.

32. Huneburg R, et al. Chromocolonoscopy detects more adenomas than white light colonoscopy or narrow band imaging colonoscopy in hereditary nonpolyposis colorectal cancer screening. Endoscopy 2009;41(4):316-22.

33. Ramsoekh D, et al. A back-to-back comparison of white light video endoscopy with autofluorescence endoscopy for adenoma detection in high-risk subjects. Gut 2010;59(6):785-93.
34. van den Broek FJ, et al. Study designs to compare new colonoscopic techniques: clinical considerations, data analysis, and sample size calculations. Endoscopy 2013;45(11):922-7.

35. Barclay RL, et al. Colonoscopic withdrawal times and adenoma detection during screening colonoscopy. N Engl J Med 2006;355(24):253341.

36. Jasperson KW, et al. Hereditary and familial colon cancer. Gastroenterology 2010;138(6):2044-58.

37. Kaltenbach T, et al. Proficiency in the diagnosis of nonpolypoid colorectal neoplasm yields high adenoma detection rates. Dig Dis Sci 2012;57(3):764-70.

38. Sanduleanu S, et al. Development of expertise in the detection and classification of non-polypoid colorectal neoplasia: Experiencebased data at an academic GI unit. Gastrointest Endosc Clin N Am 2010;20(3):449-60.

39. Sanduleanu S, et al. A roadmap to the implementation of chromoendoscopy in inflammatory bowel disease colonoscopy surveillance practice. Gastrointest Endosc 2016;83(1):213-22. 
40. Newton K, et al. Colonoscopy screening compliance and outcomes in patients with Lynch syndrome. Colorectal Dis 2015;17(1):38-46.

41. Koornstra JJ, Vasen HF. Surveillance colonoscopy practice in Lynch syndrome in the Netherlands: A nationwide survey. World J Gastroenterol 2007;13(34):4658-9. 


\section{Supplementary material}

Supplementary Table 1. Outcomes of quality assessment in the 8 studies included in the systematic review using the Quality Assessment of Diagnostic Accuracy Studies (QUADAS) tool

\begin{tabular}{|l|l|l|l|l|l|l|l|l|l|l|l|l|l|l|l|}
\hline Author & Year & $\mathbf{1}$ & $\mathbf{2}$ & $\mathbf{3}$ & $\mathbf{4}$ & $\mathbf{5}$ & $\mathbf{6}$ & $\mathbf{7}$ & $\mathbf{8}$ & $\mathbf{9}$ & $\mathbf{1 0}$ & $\mathbf{1 1}$ & $\mathbf{1 2}$ & $\mathbf{1 3}$ & $\mathbf{1 4}$ \\
\hline Hurlstone et al. $^{28}$ & 2005 & $\mathrm{Y}$ & $\mathrm{Y}$ & $\mathrm{Y}$ & $\mathrm{Y}$ & $\mathrm{Y}$ & $\mathrm{Y}$ & $\mathrm{N}$ & $\mathrm{Y}$ & $\mathrm{Y}$ & $\mathrm{N}$ & $\mathrm{Y}$ & $\mathrm{Y}$ & $\mathrm{Y}$ & $\mathrm{Y}$ \\
\hline Lecomte et al. $^{29}$ & 2005 & $\mathrm{Y}$ & $\mathrm{Y}$ & $\mathrm{Y}$ & $\mathrm{Y}$ & $\mathrm{Y}$ & $\mathrm{Y}$ & $\mathrm{N}$ & $\mathrm{Y}$ & $\mathrm{Y}$ & $\mathrm{N}$ & $\mathrm{Y}$ & $\mathrm{Y}$ & $\mathrm{Y}$ & $\mathrm{Y}$ \\
\hline Stoffel et al. ${ }^{13}$ & 2008 & $\mathrm{Y}$ & $\mathrm{Y}$ & $\mathrm{Y}$ & $\mathrm{Y}$ & $\mathrm{Y}$ & $\mathrm{Y}$ & $\mathrm{Y}$ & $\mathrm{Y}$ & $\mathrm{Y}$ & $\mathrm{N}$ & $\mathrm{Y}$ & $\mathrm{Y}$ & $\mathrm{Y}$ & $\mathrm{Y}$ \\
\hline East et al. $^{30}$ & 2008 & $\mathrm{Y}$ & $\mathrm{Y}$ & $\mathrm{Y}$ & $\mathrm{Y}$ & $\mathrm{Y}$ & $\mathrm{Y}$ & $\mathrm{N}$ & $\mathrm{Y}$ & $\mathrm{Y}$ & $\mathrm{N}$ & $\mathrm{Y}$ & $\mathrm{Y}$ & $\mathrm{Y}$ & $\mathrm{Y}$ \\
\hline Hüneburg et al. ${ }^{32}$ & 2009 & $\mathrm{Y}$ & $\mathrm{Y}$ & $\mathrm{Y}$ & $\mathrm{Y}$ & $\mathrm{Y}$ & $\mathrm{Y}$ & $\mathrm{N}$ & $\mathrm{Y}$ & $\mathrm{Y}$ & $\mathrm{N}$ & $\mathrm{N}$ & $\mathrm{Y}$ & $\mathrm{Y}$ & $\mathrm{Y}$ \\
\hline Ramsoekh et al. ${ }^{33}$ & 2010 & $\mathrm{Y}$ & $\mathrm{Y}$ & $\mathrm{Y}$ & $\mathrm{Y}$ & $\mathrm{Y}$ & $\mathrm{Y}$ & $\mathrm{Y}$ & $\mathrm{Y}$ & $\mathrm{Y}$ & $\mathrm{Y}$ & $\mathrm{N}$ & $\mathrm{Y}$ & $\mathrm{Y}$ & $\mathrm{Y}$ \\
\hline Rahmi et al. ${ }^{31}$ & 2015 & $\mathrm{Y}$ & $\mathrm{Y}$ & $\mathrm{Y}$ & $\mathrm{Y}$ & $\mathrm{Y}$ & $\mathrm{Y}$ & $\mathrm{Y}$ & $\mathrm{Y}$ & $\mathrm{Y}$ & $\mathrm{Y}$ & $\mathrm{Y}$ & $\mathrm{Y}$ & $\mathrm{Y}$ & $\mathrm{Y}$ \\
\hline
\end{tabular}


CHROMOENDOSCOPY IN LYNCH SYNDROME 



\section{CHAPTER 4}

\section{Optical diagnosis of diminutive polyps in the Dutch Bowel Cancer Screening Program; are we ready to start?}

A.J.P. van de Wetering, L.W.T. Meulen*, R.M.M. Bogie*, Q.E.W. van der Zander, A. Reumkens, B. Winkens, H.R. Cheng, J.W.A. Straathof, E. Dekker, E. Keulen, C.M. Bakker, Ch.V. Hoge, R. de Ridder, A.A.M. Masclee, S. Sanduleanu 


\section{Abstract}

\section{Introduction}

Implementation of optical diagnosis of diminutive polyps may potentially increase the efficacy and cost-effectiveness of colonoscopies. To adopt such strategy in clinical practice, the Preservation and Incorporation of Valuable endoscopic Innovations (PIVI) thresholds provide the basis to be met: $\geq 90 \%$ negative predictive value (NPV) for diagnosis of adenomatous histology and $\geq 90 \%$ agreement on surveillance intervals. We evaluated this within the Dutch Bowel Cancer Screening Program (BCSP).

\section{Patients and methods}

Endoscopic and histological data were collected from participants of the national BCSP with an unfavorable fecal immunochemical test referred for colonoscopy between February 2014 and August 2015 at four endoscopy centers. The 'resect and discard' scenario was studied, resecting diminutive polyps without histological evaluation. The agreement between optical diagnosis and histological diagnosis was measured for surveillance intervals according to Dutch, European and American post-polypectomy surveillance guideline.

\section{Results}

Fifteen certified endoscopists participated in this study and included 3028 diminutive polyps. In 2330 patients both optical and histological diagnosis were available. Optical diagnosis of diminutive polyps showed NPV of 84\% (95\% CI 8087) for adenomatous histology in the rectosigmoid. Applying the 'resect and discard' strategy resulted in $90.6 \%, 91.2 \%, 90.9 \%$ agreement on surveillance intervals for the Dutch, European and American guideline respectively.

\section{Conclusion}

Our data representing current clinical practice in the Dutch BCSP practice on optical diagnosis of diminutive polyps showed that the accuracy of predicting histology remains challenging, and the risk of incorrect optical diagnosis is still significant. Therefore, it is too early to safely implement these strategies. 


\section{Introduction}

Colorectal cancer (CRC) is a major cause of cancer-related mortality and morbidity in the Western world. ${ }^{1}$ To reduce CRC incidence and mortality, CRC screening programs have been implemented. ${ }^{2,3}$ Screening via fecal immunochemical testing (FIT) is proven to be effective in reducing CRC-related deaths. ${ }^{4}$

In 2014 the FIT-based Dutch Bowel Cancer Screening Program (BCSP) was implemented for individuals between 55 to 75 years of age. After an unfavorable FIT result, patients are invited for a colonoscopy to detect and resect (pre-) cancerous lesions. This has resulted in an increase in number of colonoscopies, polyp detection and resection and histological assessments, leading to a substantial financial burden on the health care system. ${ }^{5}$

The majority of polyps found during screening colonoscopy is small $(\leq 10 \mathrm{~mm})$ and contain non-advanced histologic features, but in current clinical practice all polyps are resected and sent for histological assessment, on which surveillance recommendations are made.

It has been seriously questioned whether histological evaluation of all these small, diminutive lesions is worthwhile and more efficient and cost-effective strategies should be implemented. ${ }^{6}$

Optical diagnosis of colorectal polyps refers to 'in vivo' estimation of histology of the polyp by endoscopists using high-definition endoscopy in conjunction with (virtual) chromoendoscopy. ${ }^{7}$ Two strategies are proposed for implementation in clinical practice, but only if the 'Preservation and Incorporation of Valuable endoscopic Innovations' (PIVI) thresholds are met. ${ }^{7}$

First, the 'resect and discard' strategy applies to diminutive $(\leq 5 \mathrm{~mm})$ colorectal adenomatous polyps which are resected, but are not sent out for histological evaluation (PIVI threshold: $\geq 90 \%$ agreement between optical diagnosis and histological diagnosis in determining the post-polypectomy surveillance interval). 
Second, the 'diagnose and leave' strategy, where diminutive hyperplastic polyps in the rectosigmoid are identified and left in situ (PIVI threshold: $\geq 90 \%$ negative predictive value (NPV) for optical diagnosis of diminutive adenomatous polyps). ${ }^{8}$

Up to now, data on optical diagnosis have been obtained mainly in study settings, i.e. from expert centers with high-confidence optical diagnosis, as the PIVI guidelines suggest. However, in order to actually implement these strategies, data from routine clinical practice are needed. Here, we present the first detailed data from the Dutch Bowel Cancer Screening Program (BCSP); a real-life but standardized endoscopy practice setting.

Aim of this study was to evaluate whether the PIVI thresholds are met regarding a) the diagnostic accuracy of optical diagnosis for diminutive polyps and regarding b) the 'resect and discard' and 'diagnose and leave' strategy, within the BCSP in a defined region of the Netherlands, South Limburg, representing our national data. ${ }^{9}, 10$ 


\section{Methods}

\section{Patients and centers}

Longitudinal data collection was performed in the four endoscopy centers in South-Limburg region of the Netherlands: one academic center and three regional endoscopy units. All endoscopic and histological data of FIT-unfavorable participants (55-75 years) that underwent colonoscopy within the context of the Dutch BCSP from February 2014 to August 2015 were collected. A threshold of $15 \mu \mathrm{g} \mathrm{Hb} / \mathrm{g}$ feces was considered FIT unfavorable (FOB gold, Sentinel, Milan, Italy) in the first six months but was raised to $47 \mathrm{Hb} / \mathrm{g}$ because of limitations in endoscopy capacity. ${ }^{5}$

We included all patients with index colonoscopies fulfilling the quality criteria in the screening program (cecal intubation and adequate bowel preparation defined as Boston Bowel Preparation Score (BBPS) $\geq 6$ ) in this retrospective analysis.

This trial is registered in the Netherlands Trial Registry (NTR4844) and the METC of Maastricht University Medical Center assigned approval for the prospective colonoscopy database (number: 14-4-046). The need for informed consent was waived by the Institutional Review Board.

\section{Endoscopists and equipment}

European guidelines for quality assurance in CRC screening have been set. ${ }^{3}$ In the Netherlands, endoscopists have to be certified before being allowed to participate in the BCSP. ${ }^{11,12}$

To be admitted to the Dutch BCSP, endoscopists should have performed at least 300 colonoscopies and over 50 polypectomies per year. Furthermore, quality measures have been set and are evaluated. ${ }^{11}$ In addition, endoscopists are required to register 100 consecutive colonoscopies with corresponding quality indicators. Then, a theoretical e-learning module should be accomplished and colonoscopic skills are evaluated in live practice setting and via videos. ${ }^{12}$ All endoscopists in this study fulfilled the quality measures for the screening program as described above but they received no specific additional training regarding optical diagnosis of colorectal polyps. 
Since the data are retrieved from a clinical practice setting, endoscopists performed standard care and were not informed about the study. All parameters currently included in the standardized endoscopy-report for the Dutch BCSP were obtained, assuming that all lesions found have been described in this report, as this is current clinical practice.

Among others, location, size, Paris-classification and predicted histology (optical diagnosis) were reported and the removed polyps were collected and sent in for histological evaluation. The classification options for estimated histology were: adenomatous polyp, hyperplastic polyp, sessile serrated lesion, carcinoma and other. No specific classification system (NICE, WASP) nor the confidence of the estimated histology are included in the standardized endoscopy-report. Therefore these data were not available for evaluation.

High-definition white light colonoscopy (HD-WLE) was used in all endoscopy units and also (virtual) chromoendoscopy was available and used upon discretion of the endoscopist. All colonoscopies were performed using endoscopic equipment containing virtual chromoendoscopy, either I-scan (Pentax Europe ß) used in one endoscopy unit or NBI (Olympus, Tokyo, Japan), used in the three other endoscopy units. The use of image-enhancement was not systematically included in the endoscopy report. To obtain an estimation on the use of image-enhancement endoscopy (IEE), we reviewed the photo documentation to see whether imageenhancement was captured in the photos. The use of IEE is scored for every polyp, and in case no photo was available or in case of more polyps in the same region, there had to be $\geq 5$ (consecutive) photos where IEE was used for a positive score.

\section{Colonoscopy}

Standard bowel preparation regimens were used with polyethylene glycol solution containing ascorbic acid or Picosulfate sodium (Moviprep® Norgine GmbH, Marburg, Germany or Picoprep®, Ferring GmbH, Kiel, Germany). After introduction to the cecum, the quality of bowel preparation was scored using the Boston Bowel Preparation Score (BBPS), where 3 is the maximum score for each segment (right, 
transverse, left) resulting in a total maximum score of $9 .{ }^{13}$ BBPS score of $\geq 2$ for each segment and $\geq 6$ in total is considered adequate bowel preparation.

\section{Histology}

All resected lesions were sent to the local pathology department and processed according to standard protocol. All pathologists had been trained and authorized for participation in the BCSP. ${ }^{11}$ The Vienna criteria for gastrointestinal epithelial neoplasia were used for classifying the biopsies, and the diagnosis by histology was used as reference. ${ }^{14}$

\section{Outcome measures and statistical analysis}

The outcome was the diagnostic accuracy, i.e. overall accuracy, sensitivity, specificity, negative predictive value (NPV) and positive predictive value (PPV) between optical diagnosis and histological diagnosis of diminutive polyps, where histological diagnosis was used as reference standard. All polyps $\leq 5 \mathrm{~mm}$ with both optical diagnosis and histological evaluation were included in the analysis. To clarify the results, the data was dichotomized into 'adenomas versus all other polyps' and 'hyperplastic polyps versus all other polyps'. Cross tables were made allowing to calculate the overall accuracy (percentage of congruent pairs), sensitivity, specificity, NPV and PPV. To take into account the use of IEE, a sensitivity analysis is performed, using Chi-square test, for the use of IEE and optical diagnosis.

To analyze whether diagnostic accuracy differs between the endoscopy units Chisquare test was used. We performed a sensitivity analysis to measure the effect of clustering (i.e. multiple lesions per patient), by calculating the values of the first primary outcome with and without multilevel correction.

The other outcome parameter was the post-polypectomy surveillance intervals based on optical diagnosis, according to a) Dutch Surveillance Guidelines ${ }^{15}$

b) European (ESGE) post-polypectomy colonoscopy surveillance guidelines ${ }^{16}$ and

c) American Guidelines (AGA) for surveillance after polypectomy. ${ }^{2}$ 
Surveillance intervals were determined per patient based on a combination of optical diagnosis (for diminutive polyps) and histology, where histology was used as reference. For each individual patient, all lesions (diminutive but also larger lesions) were taken into account when determining the interval of surveillance.

These outcomes are chosen to evaluate whether two strategies can be implemented in clinical practice. The PIVI threshold for implementing the 'resect and discard' strategy is $\geq 90 \%$ agreement between optical diagnosis and histological diagnosis in determining the post-polypectomy surveillance interval. For implementation of the 'diagnose and leave' strategy the PIVI threshold that should be met is $\geq 90 \%$ negative predictive value (NPV) for optical diagnosis of diminutive adenomatous polyps

Statistical analyses were performed using IBM SPSS Statistics for Windows Statistical Package for Social Sciences (version 22, Armonk, NY, IBM Corp.) and R-statistics was used for the sensitivity analysis ( $R$ Foundation for Statistical Computing, Vienna, Austria).

\section{Results}

\section{Patient characteristics}

Between February 2014 and August 2015, 2470 participants in the Dutch BCSP with an unfavorable FIT result underwent an index colonoscopy with polypectomy in the South Limburg region. A total of 140 cases were excluded due to insufficient colonoscopy quality (no cecal intubation $(\mathrm{n}=51)$ ), inadequate bowel preparation $(\mathrm{n}=19)$ or both $(\mathrm{n}=70)$ ) (Figure 1), resulting in 2330 patients eligible for this study. In Table 1 characteristics of the included patients are described.

Fifteen endoscopists participated in this study $(n=5$ from the academic center, $\mathrm{n}=10$ from regional endoscopy units). All had extensive colonoscopy experience (endoscopy experience in years: mean 10.9 years, SD 5.7; range 3 to 22 years) and had been certified for the national CRC screening program. The number of BCSP colonoscopies performed per endoscopist in the current study varied (mean 165 colonoscopies, SD 119; range 11 to 363 ). 


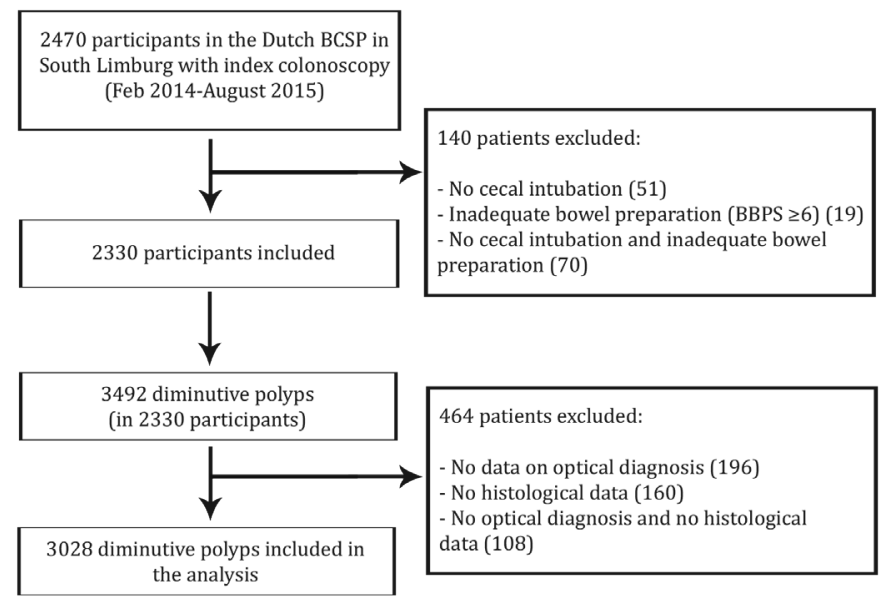

Figure 1. Flowchart of the included patients and polyps

\section{Lesion characteristics}

In total, 7369 polyps were found; 1573 were $>10 \mathrm{~mm}$ and 2304 with size $6-10 \mathrm{~mm}$. From the total of 3492 diminutive polyps, both optical diagnosis ( $\mathrm{n}=196$ missing) and histological data ( $\mathrm{n}=160$ missing) needed to be available ( $\mathrm{n}=108$ both missing), resulting in 3028 diminutive lesions that were included (Figure 1). Endoscopic characteristics of these polyps are shown in Table 2. The median size of the diminutive polyps was $4 \mathrm{~mm}, 40 \%$ of the polyps were located in rectosigmoid $(n=1222)$. Histology showed that $67 \%$ were adenomatous and $19 \%$ hyperplastic. In the 1-5mm group, a total of 3 carcinomas were detected and 15 adenomas showed high-grade dysplasia (Table 2).

\section{Performance of optical diagnosis}

Optical diagnosis for diminutive adenomas in the colon and rectum showed a diagnostic accuracy of $76 \%$ (95\% CI 74-77) compared to histological diagnosis. The negative predictive value (NPV) for adenomatous histology was 69\% (95\% CI 6673) (Table 3). In the rectosigmoid, a total of 1222 diminutive lesions were found, the NPV for adenomatous histology was 84\% (95\% CI 80-87). For hyperplastic polyps in the rectosigmoid the NPV was 76\% (95\% CI 73-78), the PPV was $61 \%$ (95\% CI 56-66) and overall accuracy was 71\% (95\% CI 69-74) (Table 3). 
CHAPTER 4

Table 1. Characteristics of the included patients $(n=2330)$

\begin{tabular}{|l|c|}
\hline Age (mean, SD), years & $68(5)$ \\
\hline Gender (female, $\mathrm{n}(\%))$ & $889(39)$ \\
\hline ASA classification (n, \%) & $801(34)$ \\
1 & $1441(62)$ \\
2 & $88(4)$ \\
3 & $1(0)$ \\
\hline Boston Bowel Preparation Score (mean, SD)* & $9(1)$ \\
\hline Cecal withdrawal time (mean, SD), minutes & $17(11)$ \\
\hline *Only patients with cecal intubation and BBPS $\geq 6$ were included \\
\hline
\end{tabular}

Table 3. Optical diagnosis versus histological evaluation of diminutive polyps

\begin{tabular}{|l|c|c|}
\hline & \multicolumn{2}{|c|}{$\begin{array}{c}\text { Lesions in colon and rectum } \\
\text { (n=3028* }\end{array}$} \\
\hline & $\begin{array}{c}\text { Adenomas } \\
\text { (n=2038)** }\end{array}$ & $\begin{array}{c}\text { Hyperplastic polyps } \\
\text { (n=563)** }\end{array}$ \\
\hline Overall accuracy (95\% CI) & $76 \%(74-77)$ & $79 \%(77-80)$ \\
\hline Sensitivity (95\% CI) & $90 \%(88-91)$ & $48 \%(44-53)$ \\
\hline Specificity (95\% CI) & $47 \%(44-50)$ & $85 \%(84-87)$ \\
\hline Positive Predictive Value (PPV) $(95 \% \mathrm{CI})$ & $78 \%(76-79)$ & $43 \%(39-47)$ \\
\hline Negative Predictive Value (NPV) $(95 \% \mathrm{CI})$ & $69 \%(66-73)$ & $88 \%(86-89)$ \\
\hline & \multicolumn{2}{|c|}{ Lesions in the rectosigmoid } \\
(n=1222)*
\end{tabular}


Table 2. Endoscopic and histologic characteristics of the diminutive lesions and the accuracy per center

\begin{tabular}{|c|c|c|}
\hline & $\begin{array}{l}\text { Lesions in colon and } \\
\text { rectum }\end{array}$ & $\begin{array}{l}\text { Lesions in } \\
\text { rectosigmoid }\end{array}$ \\
\hline Number of diminutive lesions & 3028 & 1222 \\
\hline Polyp size (mean, SD) in mm & $4(1)$ & $4(1)$ \\
\hline $\begin{array}{l}\text { Polyp size (n, \%) } \\
1-2 \mathrm{~mm} \\
3-5 \mathrm{~mm}\end{array}$ & $\begin{array}{c}544(18) \\
2484(82)\end{array}$ & $\begin{array}{c}192(16) \\
1030(84)\end{array}$ \\
\hline $\begin{array}{l}\text { Paris classification (n, \%)* } \\
\text { Ip } \\
\text { Is } \\
\text { IIa } \\
\text { IIb } \\
\text { Unclassified }\end{array}$ & $\begin{array}{c}235(8) \\
2477(82) \\
264(9) \\
15(0) \\
37(1)\end{array}$ & $\begin{array}{c}118(10) \\
985(81) \\
95(8) \\
4(0) \\
20(1)\end{array}$ \\
\hline $\begin{array}{l}\text { Histology (n, \%) } \\
\text { Adenoma } \\
\quad \text { Tubular } \\
\text { Villous } \\
\text { Tubulovillous } \\
\text { Sessile serrated lesion or traditional serrated adenoma } \\
\text { Hyperplastic polyp } \\
\text { Carcinoma } \\
\text { Other finding } \\
\text { No abnormality }\end{array}$ & $\begin{array}{c}2038(67) \\
1964 \\
1 \\
73 \\
106(4) \\
563(19) \\
3(0) \\
99(3) \\
222(7)\end{array}$ & $\begin{array}{c}602(49) \\
572 \\
1 \\
29 \\
41(3) \\
439(36) \\
2(0) \\
48(4) \\
92(8)\end{array}$ \\
\hline $\begin{array}{l}\text { Dysplasia (n, \%) } \\
\text { For adenomas } \\
\text { - Low-grade dysplasia } \\
\text { - High-grade dysplasia } \\
\text { - Unclassified } \\
\text { For sessile serrated lesions } \\
\text { - With dysplasia } \\
\text { - Without dysplasia } \\
\text { - Unclassified }\end{array}$ & $\begin{array}{c}2022(99.2) \\
15(0.7) \\
1(0.1) \\
\\
31(29.2) \\
71(67.0) \\
4(3.8)\end{array}$ & $\begin{array}{c}589(97.8) \\
12(2.0) \\
1(0.2) \\
\\
10(24.4) \\
30(73.2) \\
1(2.4)\end{array}$ \\
\hline $\begin{array}{l}\text { Diagnostic accuracy per endoscopy center } \\
\text { (n of polyps, \% correctly estimated lesions) } \\
\text { Center } 1^{* *} \\
\text { Center } 2^{* *} \\
\text { Center } 3^{* *} \\
\text { Center } 4^{* *}\end{array}$ & $\begin{array}{l}\text { Adenomas in colon and } \\
\text { rectum } \\
839(77) \\
1007(74) \\
928(77) \\
254(76)\end{array}$ & $\begin{array}{l}\text { Hyperplastic polyps in } \\
\text { rectosigmoid } \\
\\
339(72) \\
397(70) \\
386(73) \\
100(70)\end{array}$ \\
\hline
\end{tabular}


A total of 150 polyps in rectosigmoid (12.3\% of the total) were optically misdiagnosed as hyperplastic. In $5.1 \%$ and $1.9 \%$ of the cases, an adenoma or sessile serrated lesion, respectively, would have been left in place (5.3\% other/no abnormality) (Table 4 ). For the optically misdiagnosed lesions ( $n=139 / 150$ photo documentation available), no significant difference was found with regard to use of IEE $(p=0.620)$.

Diagnostic accuracy for diminutive adenomas in the colon and rectum ranged from 74 to $78 \%(p=0.393)$ between the four endoscopy units and regarding hyperplastic lesions in the rectosigmoid diagnostic accuracy ranged from 70 to $73 \%(p=0.769)$ (Table 2). The overall diagnostic accuracy between the fifteen endoscopists ranged from $69 \%$ to $87 \%$. From 2576 polyps photo documentation was available. Image enhancement had been documented by endoscopy photos in $36.9 \%$, where in the majority of the cases I-scan was used.

Table 4. Specification of the polyps incorrectly estimated as hyperplastic polyp in the rectosigmoid region

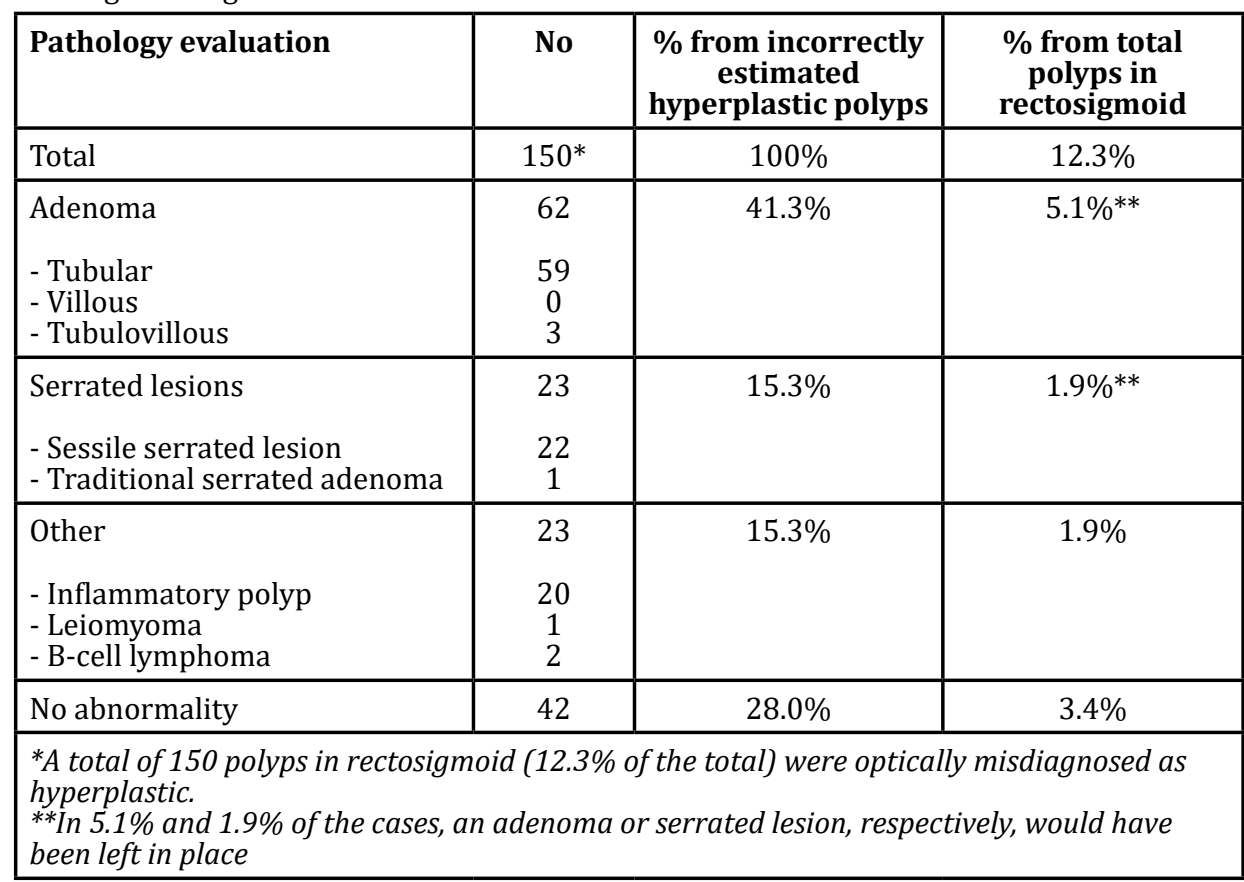


There was no significant difference between the use of IEE and the correct optical diagnosis for both adenomas in the colon and rectum $(p=0.612)$ and for hyperplastic polyps in the rectosigmoid ( $p=0.842)$. The sensitivity analysis to correct for clustering (i.e. multiple lesions per patient) showed similar results. (Data not shown)

\section{Surveillance intervals}

In Table 5 results of the surveillance intervals are given. Surveillance intervals have been calculated at patient level, meaning that if only diminutive polyps were found the surveillance interval is based on optical diagnosis solely, whereas if additional polyps ( $>5 \mathrm{~mm}$ ) were found, the histology of these non-diminutive polyps determined the surveillance intervals. For the 'resect and discard' strategy agreement for the Dutch, European and American guidelines was 90.6\%, 91.2\% and 90.9\% respectively. Approximately $6.0 \%$ would have received a shorter surveillance interval based on optical diagnosis, while in $2.8 \%$ to $3.3 \%$ of the cases a longer surveillance interval would have been recommended.

A detailed overview of the surveillance intervals for the 'resect and discard' and 'diagnose and leave' strategies using different guidelines is presented in Supplementary Table 1 and Supplementary Table 2.

Table 5. Surveillance intervals based on optical diagnosis vs. histology, according to different guidelines (NL, EU, USA) and applying the 'resect and discard' scenario.

\begin{tabular}{|l|c|c|c|}
\hline & $\begin{array}{c}\text { Agreement between } \\
\text { optical diagnosis and } \\
\text { histology }\end{array}$ & $\begin{array}{c}\text { Surveillance } \\
\text { earlier }\end{array}$ & $\begin{array}{c}\text { Surveillance } \\
\text { later* }\end{array}$ \\
\hline $\begin{array}{l}\text { Dutch } \\
\text { guideline }\end{array}$ & $\begin{array}{c}90.6 \% \\
\mathrm{n}=2110\end{array}$ & $\begin{array}{c}6.2 \% \\
\mathrm{n}=144\end{array}$ & $\begin{array}{c}3.3 \% \\
\mathrm{n}=76\end{array}$ \\
\hline $\begin{array}{l}\text { European } \\
\text { guideline }^{16}\end{array}$ & $91.2 \%$ & $5.9 \%$ & $2.9 \%$ \\
$\mathrm{n}=6126$ & $\mathrm{n}=137$ & $2.8 \%$ \\
\hline American $_{\text {guideline }}{ }^{2}$ & $90.9 \%$ & $6.2 \%$ & $\mathrm{n}=66$ \\
\hline *This includes also the patients who receive no surveillance according to optical \\
diagnosis. The number of patients who would receive no surveillance are for the Dutch \\
guideline 36/76 patients, for the European guideline 36/67 patients and according to the \\
American guideline 4/66.
\end{tabular}




\section{Discussion}

We have evaluated the accuracy of optical diagnosis of diminutive polyps, as well as the scenarios for 'resect and discard' and 'diagnose and leave' in the clinical endoscopy practice setting of the Bowel Cancer Screening Program (BCSP) in the Netherlands. Optical diagnosis of diminutive adenomatous polyps in the rectosigmoid showed 72\% diagnostic accuracy and 84\% NPV: thus, the PIVI thresholds were not met.

When applying the 'resect and discard' scenario, agreement on surveillance intervals between optical and histological diagnosis applying the Dutch, European and American surveillance guidelines was 90.6\%, 91.2\% and 90.9\% respectively. Therefore, at group level, the PIVI thresholds ( $\geq 90 \%$ agreement) concerning surveillance strategies were met.

Given the substantial amount of research focusing on optical diagnosis and the potential cost savings, this is an important and clinically relevant topic. ${ }^{17,18}$ However, results of studies assessing optical diagnosis of small and diminutive polyps vary considerably. So far, data have been obtained predominantly in well controlled study settings, where endoscopists were additionally trained in recognition and characterization of lesions and had been instructed on the systematic use of imageenhancement. The baseline characteristics of the diminutive lesions in our study are within the range of variation reported in recent literature, and are therefore representative for national and global data. ${ }^{19,20}$

When evaluating published data of additionally trained endoscopists, the NPV for optical diagnosis of adenomas in the rectosigmoid varies from 82.0 to $94.7 \%$ in studies where narrow-band imaging (NBI) was used..$^{21}$ Ladabaum et al. ${ }^{22}$ showed that while only $25 \%$ of the trained endoscopists used NBI, polyps were assessed with over $90 \%$ accuracy.

Image enhancement for optical diagnosis of diminutive polyps is considered to be beneficial, but remains an item of discussion since several studies have not shown 
significant differences in accuracy for optical diagnosis with image enhancement compared to HD-WLE. ${ }^{23-25}$ In our study, reflecting daily endoscopy practice, use of image-enhancement in addition to HD-WLE was left at the discretion of the endoscopist. In 36.9\% use of image-enhancement was photo-documented and no significant differences were found in optical diagnosis with or without use of IEE.

Experience and additional training of the endoscopists may substantially add to the accuracy of optical diagnosis. Endoscopists working in academic centers obtain better results in optical diagnosis compared to endoscopists working in community practices. ${ }^{22}$ Indeed, in a surveillance setting in non-academic centers without additional training, Kuiper et al. ${ }^{26}$ noted low sensitivity (77.0\%) and specificity (78.8\%) for optical diagnosis.

In our study, the performance of academic and regional centers with respect to optical diagnosis was in the same range. Concerning surveillance intervals, in previous studies 19\% inaccuracy in determining surveillance intervals based on optical diagnosis has been reported. ${ }^{26}$ It should be noted that surveillance intervals were calculated on patient level, therefore all polyps (diminutive but also larger polyps) were taken into account, so it should be noted that intervals are mostly affected by the larger polyps. Therefore, optical misdiagnosis of smaller polyps can be overruled by the presence of larger polyps. This raises the question whether surveillance interval is the most appropriate criterium when deciding on diminutive polyps. It does however perfectly represent the impact of the guidelines used in current clinical practice.

A recent Dutch study from Vleugels et al. has shown that at group level in a selected population of endoscopists after additional training, optical diagnosis of diminutive polyps (with high-confidence) in the Dutch FIT-based CRC screening setting using narrow-band imaging (NBI) met the ASGE PIVI thresholds. ${ }^{20}$ However, at individual level, only $59 \%$ of the additionally trained endoscopists did meet these PIVI thresholds. These authors showed that selected endoscopists, additionally trained by a validated training module on $\mathrm{NICE}^{27}$ and $\mathrm{WASP}^{28}$ were able to diagnose 
neoplastic lesions (with high-confidence) using NBI in the rectosigmoid with pooled NPVs of more than $90 \% .{ }^{20}$ In addition, they were also able to accurately recommend surveillance intervals based on optical diagnosis. ${ }^{20}$ When interpreting these data, it should be noted that these endoscopists represent an expert group, of which endoscopists were only allowed to participate after passing an additional exam ( $\geq 90 \%$ diagnostic accuracy (same as in PIVI)). ${ }^{20}$ Therefore, the results of that study cannot be extrapolated directly to community practice. On the other hand, Vleugels et al. ${ }^{20}$ have clearly shown that optical diagnosis may become feasible in a special setting in which endoscopist training and feedback is incorporated.

In a study by Schachschal et al. ${ }^{29}$ performed in a screening setting, optical diagnosis had an accuracy of only $71.1 \%$ and NPV of 59.3\%. Our results compare favorably with that study with NPV for hyperplastic polyps in the rectosigmoid and for adenomas in the colon of respectively $76 \%$ and $69 \%$. The agreement on surveillance intervals in our study reached an accuracy of over $90 \%$, while data from the Schachschal et al. study cannot be retrieved from the manuscript. ${ }^{29}$

In order to implement these strategies in clinical practice, costs should be considered. Using simulation modelling, optical diagnosis in the Dutch BCSP appears to save costs without decreasing program effectiveness when compared with current histology analysis of all diminutive polyps. ${ }^{30}$ In line with these modelling data, Hassan et al. have already shown that the 'resect and discard' strategy for diminutive polyps detected during screening indeed results in economic benefit without impact on program efficacy. ${ }^{6}$ Applying these strategies may not only result in cost savings but also in a reduction of risks of polypectomies and of patient discomfort.

In the case that lesions are left in situ (i.e. 'diagnose and leave' scenario), an incorrect optical diagnosis may have significant impact. In our study twelve percent of the rectosigmoid lesions was estimated as hyperplastic but contained other histology (i.e. adenomas and serrated polyps). 
When the lesions are removed (i.e. 'resect and discard' scenario), the impact of incorrect optical diagnosis is limited.

The high-risk lesions found in our study (3 carcinomas and 15 lesions with highgrade dysplasia) should be considered carefully. Here, evaluation of treatment and resection margins is of importance, and they should receive stricter follow-up.

Several strengths of our study need to be acknowledged. First, we evaluated the efficacy of the optical diagnosis strategy within a) the structured setting of the nationwide Bowel Cancer Screening Program b) regular endoscopy practices where all participating endoscopists were qualified and accredited for performing colonoscopies for the Dutch FIT-based BCSP ${ }^{12}$, but without additional training or selection for competency in optical diagnosis. We prospectively collected data from four endoscopy units (both academic and regional) in South Limburg (the Netherlands). The results therefore reflect daily clinical practice in the Netherlands in the first years of implementation of the BCSP.

Several limitations need to be acknowledged as well. Since standardized endoscopy reports are used for data collection, some detailed information is lacking. Therefore, the results of this study should be interpreted with caution.

First, the level of confidence with which an endoscopist rates his/her optical diagnosis is relevant. A meta-analysis from 2015 showed that estimations with highconfidence are more likely to be correct. ${ }^{7}$ In our real-life study endoscopists neither were asked for nor included the level of confidence in the standard endoscopy-report and we were therefore not able to assess the level of confidence for optical diagnosis.

Second, image-enhancement was used upon discretion of the endoscopist, but the specific use per polyp was not reported. Based on photo-documentation, imageenhancement was used in at least $36.9 \%$ of endoscopies. 
To improve performance and to allow implementation of optical diagnosis in the setting of a national BCSP, essential steps need to be taken 1) for equipment: standard use of high-definition white light endoscopy with additional image enhancement 2) for endoscopists: additional training and monitoring of individual performance 3) standard use of optical classification systems (e.g. NICE or WASP) 4) inclusion of 'the level of confidence in optical diagnosis' of the endoscopist in the optical diagnosis algorithm 5) photo documentation and archiving. ${ }^{31,32}$

Implementation of optical diagnosis strategies in clinical practice remains challenging. ${ }^{31}$ A simplified approach has been suggested by Atkinson and East ${ }^{33}$; the DISCARD-lite strategy where all diminutive polyps proximal to rectosigmoid junction are assumed premalignant and therefore 'resect and discard' is applied, while hyperplastic polyps in the rectosigmoid can be left in situ. A recent study by von Renteln et al. indicates that this simplified combined optical and location-based strategy may help to overcome current challenges in the implementation of the 'resect and discard' strategy. ${ }^{34}$

In the near future an important role for artificial intelligence (AI) in optical detection and characterization of diminutive polyps is foreseen, thus reducing or even eliminating endoscopist inter-observer variability. Several computer aided detection and characterization systems and algorithms are being developed with promising preliminary data such as a NPV for identification and classification of diminutive rectosigmoid adenomas ranging from 91.5\%-97\%. ${ }^{35-38}$ More extensive research in larger clinical trial settings is necessary to confirm and expand on these results. 
Based on our data from regular endoscopy care in the BCSP, we cannot recommend to leave diminutive rectosigmoid polyps in place. On the other hand, the thresholds for the 'resect and discard' strategy, i.e. agreement on post-polypectomy surveillance intervals were met. Implementation of this strategy can therefore be considered. These results however, need to be validated, in a setting where the above mentioned steps have been implemented (i.e. standardized and structural use of level of confidence and use of IEE).

To conclude, our study representing current clinical practice in the Dutch BCSP practice on optical diagnosis of diminutive polyps showed that the accuracy of predicting histology remains challenging, and the risk of incorrect optical diagnosis is significant. Therefore, it is too early to safely implement these strategies. It remains to be determined whether optical diagnosis will structurally meet the PIVI criteria in routine clinical endoscopy practices. 


\section{References}

1. Edwards BK, et al. Annual report to the nation on the status of cancer, 1975-2006, featuring colorectal cancer trends and impact of interventions (risk factors, screening, and treatment) to reduce future rates. Cancer 2010;116(3):544-73.

2. Lieberman DA, et al. Guidelines for colonoscopy surveillance after screening and polypectomy: a consensus update by the US Multi-Society Task Force on Colorectal Cancer. Gastroenterology 2012;143(3):844-57.

3. European Colorectal Cancer Screening Guidelines Working Group, et al. European guidelines for quality assurance in colorectal cancer screening and diagnosis: overview and introduction to the full supplement publication. Endoscopy 2013;45(1):51-9.

4. Shaukat A, et al. Long-term mortality after screening for colorectal cancer. N Engl J Med 2013;369(12):110614.

5. Toes-Zoutendijk E, et al. Real-Time Monitoring of Results During First Year of Dutch Colorectal Cancer Screening Program and Optimization by Altering Fecal Immunochemical Test CutOff Levels. Gastroenterology 2017;152(4):767-75 e2.

6. Hassan C, et al. A resect and discard strategy would improve costeffectiveness of colorectal cancer screening. Clin Gastroenterol Hepatol 2010;8(10):865-9, 9 e1-3.
7. ASGE Committee, et al. ASGE Technology Committee systematic review and meta-analysis assessing the ASGE PIVI thresholds for adopting real-time endoscopic assessment of the histology of diminutive colorectal polyps. Gastrointest Endosc 2015;81(3):502 e1- e16.

8. Rex DK, et al. The American Society for Gastrointestinal Endoscopy PIVI (Preservation and Incorporation of Valuable Endoscopic Innovations) on real-time endoscopic assessment of the histology of diminutive colorectal polyps. Gastrointest Endosc 2011;73(3):419-22.

9. le Clercq CM, et al. Postcolonoscopy colorectal cancers are preventable: a population-based study. Gut 2014;63(6):957-63.

10. Rondagh EJ, et al. Endoscopic appearance of proximal colorectal neoplasms and potential implications for colonoscopy in cancer prevention. Gastrointest Endosc 2012;75(6):1218-25.

11. RIVM. Protocol voor de toelating en auditing van coloscopiecentra en endoscopisten - Bevolkingsonderzoek Darmkanker. 2012.

12. Bronzwaer MES, et al. Quality assurance of colonoscopy within the Dutch national colorectal cancer screening program. Gastrointest Endosc 2019;89(1):1-13. 
13. Calderwood AH, et al. Boston Bowel Preparation Scale scores provide a standardized definition of adequate for describing bowel cleanliness. Gastrointest Endosc 2014;80(2):269-76.

14. Schlemper RJ, et al. The Vienna classification of gastrointestinal epithelial neoplasia. Gut 2000;47(2):251-5.

15. NVMDL. Dutch Guideline Colonoscopy Surveillance 2013.

16. Hassan C, et al. Post-polypectomy colonoscopy surveillance: European Society of Gastrointestinal Endoscopy (ESGE) Guideline. Endoscopy 2013;45(10):842-51.

17. Lieberman D, et al. Polyp size and advanced histology in patients undergoing colonoscopy screening: implications for CT colonography. Gastroenterology 2008;135(4):1100-5.

18. Von Renteln D, et al. Current standards and new developments of colorectal polyp management and resection techniques. Expert Rev Gastroenterol Hepatol 2017;11(9):835-42.

19. Vleugels JLA, et al. Diminutive Polyps With Advanced Histologic Features Do Not Increase Risk for Metachronous Advanced Colon Neoplasia. Gastroenterology 2019;156(3):623-34 e3.
20. Vleugels JLA, et al. Effects of Training and Feedback on Accuracy of Predicting Rectosigmoid Neoplastic Lesions and Selection of Surveillance Intervals by Endoscopists Performing Optical Diagnosis of Diminutive Polyps. Gastroenterology 2018.

21. Ignjatovic $\mathrm{A}$, et al. Optical diagnosis of small colorectal polyps at routine colonoscopy (Detect InSpect ChAracterise Resect and Discard; DISCARD trial): a prospective cohort study. Lancet Oncol 2009;10(12):1171-8.

22. Ladabaum U, et al. Real-time optical biopsy of colon polyps with narrow band imaging in community practice does not yet meet key thresholds for clinical decisions. Gastroenterology 2013;144(1):81-91.

23. Rees CJ, et al. Narrow band imaging optical diagnosis of small colorectal polyps in routine clinical practice: the Detect Inspect Characterise Resect and Discard 2 (DISCARD 2) study. Gut 2017;66(5):887-95.

24. Klare P, et al. Narrow-band imaging vs. high definition white light for optical diagnosis of small colorectal polyps: a randomized multicenter trial. Endoscopy 2016;48(10):90915.

25. Basford PJ, et al. High-definition endoscopy with i-Scan for evaluation of small colon polyps: the HiSCOPE study. Gastrointest Endosc 2014;79(1):111-8. 
26. Kuiper T, et al. Accuracy for optical diagnosis of small colorectal polyps in nonacademic settings. Clin Gastroenterol Hepatol 2012;10(9):1016-20; quiz e79

27. Repici A, et al. Narrow-band Imaging International Colorectal Endoscopic Classification to predict polyp histology: REDEFINE study (with videos). Gastrointest Endosc 2016;84(3):479-86 e3.

28. IJspeert JE, et al. Development and validation of the WASP classification system for optical diagnosis of adenomas, hyperplastic polyps and sessile serrated adenomas/polyps. Gut 2016;65(6):963-70.

29. Schachschal G, et al. Endoscopic versus histological characterisation of polyps during screening colonoscopy. Gut 2014;63(3):45865.

30. Vleugels JLA, et al. Implementation of an optical diagnosis strategy saves costs and does not impair clinical outcomes of a fecal immunochemical test-based colorectal cancer screening program. Endosc Int Open 2017;5(12):E1197-E207.

31. Kaltenbach $\mathrm{T}$, et al. Implementation of optical diagnosis for colorectal polyps: standardization of studies is needed. Clin Gastroenterol Hepatol 2015;13(1):6-10 e1.
32. Kaminski MF, et al. Advanced imaging for detection and differentiation of colorectal neoplasia: European Society of Gastrointestinal Endoscopy (ESGE) Guideline. Endoscopy 2014;46(5):435-49.

33. Atkinson NS, East JE. Optical biopsy and sessile serrated polyps: Is DISCARD dead? Long live DISCARD-lite! Gastrointest Endosc 2015;82(1):118-21.

34. von Renteln D, et al. Simplifying Resect and Discard Strategies for Real-Time Assessment of Diminutive Colorectal Polyps. Clin Gastroenterol Hepatol 2018;16(5):706-14.

35. Mori Y, et al. Real-Time Use of Artificial Intelligence in Identification of Diminutive Polyps During Colonoscopy: A Prospective Study. Ann Intern Med 2018;169(6):357-66.

36. Byrne MF, et al. Real-time differentiation of adenomatous and hyperplastic diminutive colorectal polyps during analysis of unaltered videos of standard colonoscopy using a deep learning model. Gut 2017.

37. Chen PJ, et al.Accurate Classification of Diminutive Colorectal Polyps Using Computer-Aided Analysis. Gastroenterology 2018;154(3):568.75.

38. Vinsard DG, et al. Quality assurance of computer-aided detectionand diagnosis in colonoscopy. Gastrointest Endosc 2019;90(1):55-63. 


\section{Supplementary material}

Supplementary Table 1a. Surveillance intervals based on optical diagnosis vs. histology, according to the Dutch surveillance guideline (2012), applying the 'resect and discard' strategy*

\begin{tabular}{|c|c|c|c|c|c|c|}
\hline \multicolumn{7}{|c|}{ Surveillance intervals based on histology } \\
\hline \multirow{5}{*}{$\begin{array}{c}\text { Surveillance } \\
\text { intervals based on } \\
\text { optical diagnosis }\end{array}$} & & 3 year & 5 year & 10 year & No surveillance & Total \\
\hline & 3 year & 584 & 38 & 16 & 0 & 638 \\
\hline & 5 year & 9 & 612 & 39 & 19 & 679 \\
\hline & 10 year & 9 & 22 & 384 & 32 & 447 \\
\hline & $\begin{array}{l}\text { No } \\
\text { surveillance }\end{array}$ & 0 & 19 & 17 & 530 & 566 \\
\hline & Total & 602 & 691 & 456 & 581 & 2330 \\
\hline
\end{tabular}

Supplementary Table 1b. Surveillance intervals based on optical diagnosis vs. histology, according to the ESGE (European) surveillance guideline (2013), applying the 'resect and discard' strategy*

\begin{tabular}{|c|l|c|c|c|c|}
\hline \multicolumn{7}{|c|}{} & 3 year & 10 year & No surveillance & Total \\
\hline & 3 year & $\mathbf{9 0 3}$ & 81 & 2 & 986 \\
\cline { 2 - 6 } $\begin{array}{c}\text { Surveillance } \\
\text { intervals based on } \\
\text { optical diagnosis }\end{array}$ & 10 year & 31 & $\mathbf{6 8 4}$ & 54 & 769 \\
\cline { 2 - 6 } & $\begin{array}{l}\text { No } \\
\text { surveillance }\end{array}$ & 3 & 33 & $\mathbf{5 3 9}$ & 575 \\
\hline & Total & 937 & 798 & 595 & $\mathbf{2 3 3 0}$ \\
\hline \multirow{2}{*}{$\begin{array}{l}\text { *For this strategy optical diagnosis was used for polyps } \leq 5 \text { mm in the entire colon while for all other polyps histological } \\
\text { evaluation was used to determine surveillance intervals }\end{array}$}
\end{tabular}


Supplementary Table 1c. Surveillance intervals based on optical diagnosis vs. histology, according to the $\boldsymbol{A G A}$ (American) surveillance guideline (2012), applying the 'resect and discard' strategy*

\begin{tabular}{|c|l|c|c|c|c|c|c|}
\hline \multicolumn{2}{|c|}{} & 3 year & 5 year & $5-10$ year & 10 year & No surveillance & Total \\
\hline \multirow{4}{*}{$\begin{array}{c}\text { Surveillance } \\
\text { intervals based } \\
\text { on } \\
\text { optical } \\
\text { diagnosis }\end{array}$} & 3 year & $\mathbf{1 1 7 5}$ & 35 & 39 & 1 & 0 & 1250 \\
\cline { 2 - 9 } & 5 year & 5 & $\mathbf{2 7}$ & 2 & 0 & 0 & 34 \\
\cline { 2 - 9 } & $5-10$ years & 16 & 3 & $\mathbf{4 0 8}$ & 35 & 17 & 479 \\
\cline { 2 - 8 } & 10 year & 5 & 5 & 28 & $\mathbf{6 5}$ & 16 & 119 \\
\cline { 2 - 8 } & $\begin{array}{l}\text { No } \\
\text { surveillance }\end{array}$ & 1 & 0 & 2 & 1 & $\mathbf{4 4 4}$ & 448 \\
\hline & Total & 1202 & 70 & 479 & 102 & 477 & $\mathbf{2 3 3 0}$ \\
\hline \multirow{2}{*}{$\begin{array}{c}\text { *For this strategy optical diagnosis was used for polyps } \leq 5 \mathrm{~mm} \text { in the entire colon while for all other polyps histological } \\
\text { evaluation was used to determine surveillance intervals }\end{array}$}
\end{tabular}

Supplementary Table 2a. Surveillance intervals based on optical diagnosis vs. histology, according to the Dutch surveillance guideline (2012), applying the 'diagnose and leave' strategy*

\begin{tabular}{|c|c|c|c|c|c|c|}
\hline \multicolumn{7}{|c|}{ Surveillance intervals based on histology } \\
\hline \multirow{6}{*}{$\begin{array}{c}\text { Surveillance } \\
\text { intervals based on } \\
\text { optical diagnosis }\end{array}$} & & 3 year & 5 year & 10 year & No surveillance & Total \\
\hline & 3 year & 601 & 13 & 0 & 0 & 614 \\
\hline & 5 year & 1 & 669 & 30 & 2 & 702 \\
\hline & 10 year & 0 & 7 & 412 & 34 & 453 \\
\hline & $\begin{array}{l}\text { No } \\
\text { surveillance }\end{array}$ & 0 & 2 & 14 & 545 & 561 \\
\hline & Total & 602 & 691 & 456 & 581 & 2330 \\
\hline
\end{tabular}


Supplementary Table 2b. Surveillance intervals based on optical diagnosis vs. histology, according to the ESGE (European) surveillance guideline (2013), applying the 'diagnose and leave' strategy*

\begin{tabular}{|c|c|c|c|c|c|}
\hline \multicolumn{6}{|c|}{ Surveillance intervals based on histology } \\
\hline \multirow{4}{*}{$\begin{array}{c}\text { Surveillance } \\
\text { intervals based on } \\
\text { optical diagnosis }\end{array}$} & & 3 year & 10 year & No surveillance & Total \\
\hline & 3 year & 928 & 40 & 0 & 968 \\
\hline & 10 year & 9 & 742 & 38 & 789 \\
\hline & $\begin{array}{l}\text { No } \\
\text { surveillance }\end{array}$ & 0 & 16 & 557 & 573 \\
\hline & Total & 937 & 798 & 595 & 2330 \\
\hline
\end{tabular}

Supplementary Table 2c. Surveillance intervals based on optical diagnosis vs. histology, according to the $\boldsymbol{A G A}$ (American) surveillance guideline (2012), applying the 'diagnose and leave' strategy*

\begin{tabular}{|c|l|c|c|c|c|c|c|}
\hline \multicolumn{2}{|c|}{} & 3 year & 5 year & $5-10$ year & 10 year & No surveillance & Total \\
\hline \multirow{4}{*}{$\begin{array}{c}\text { Surveillance } \\
\text { intervals based } \\
\text { on } \\
\text { optical } \\
\text { diagnosis }\end{array}$} & 3 year & $\mathbf{1 1 9 3}$ & 15 & 23 & 0 & 0 & 1231 \\
\cline { 2 - 9 } & 5 year & 2 & $\mathbf{5 3}$ & 0 & 0 & 0 & 55 \\
\cline { 2 - 9 } & $5-10$ years & 6 & 0 & $\mathbf{4 4 1}$ & 29 & 9 & 485 \\
\cline { 2 - 8 } & 10 year & 0 & 2 & 14 & $\mathbf{7 2}$ & 15 & 103 \\
\cline { 2 - 8 } & $\begin{array}{l}\text { No } \\
\text { surveillance }\end{array}$ & 1 & 0 & 1 & 1 & $\mathbf{4 5 3}$ & 456 \\
\hline & Total & 1202 & 70 & 479 & 102 & 477 & $\mathbf{2 3 3 0}$ \\
\hline \multirow{2}{*}{$\begin{array}{l}\text { *For this strategy optical diagnosis was used for polyps } \leq 5 \text { mm in the rectosigmoid while for all other polyps } \\
\text { histological evaluation was used to determine surveillance intervals }\end{array}$}
\end{tabular}





\section{CHAPTER 5}

\section{Optical diagnosis of T1 CRCs and treatment consequences in the Dutch CRC screening programme}

Alouisa J.P. van de Wetering*, Lonne W.T. Meulen*, Marie-Eline P.H. Debeuf, Zlatan Mujagic, Ad A.M. Masclee

*Shared first authorship

Gut 2020;0:1-2.

Published Online First 14 January 2020 
The implementation of the Dutch Bowel Cancer Screening Programme (BCSP) has led towards more frequent diagnosis of early AJCC (American Joint Committee on Cancer) stage I colorectal cancers (CRC). ${ }^{1}$ In the Netherlands, screen-detected CRCs $(48.2 \%)$ in a FIT-based screening program are more often detected in an early stage compared to symptom-detected CRCs (16.7\%). ${ }^{1}$

Given the shift towards CRCs detection in earlier stages, it is important to optimize diagnosis and thereby optimize the management and treatment for these early cancer patients.

In optimizing management for T1 CRC the first crucial step is the recognition; accurate optical diagnosis. However, recognition of T1 CRC is challenging.

Backes et al. recently published an article on the pre-resection accuracy of realtime optical diagnosis of T1 colorectal cancer (T1CRC) in large non-pedunculated colorectal polyps. ${ }^{2}$ In this multicenter, prospective study, the authors developed and validated the OPTICAL model, in which a sensitivity of 78.7\% (95\% CI 64.3-89.3) for optical diagnosis of T1CRC was obtained.

Estimating the risk of a T1CRC is crucial to determine the optimal treatment strategy, and to select cases for more elaborative and expensive endoscopic en-bloc resection techniques such as endoscopic submucosal dissection (ESD), transanal minimally invasive surgery (TAMIS) or endoscopic full thickness resection (eFTR).

Current studies mainly report on the outcomes of advanced imaging by expert centers with dedicated endoscopists ${ }^{3,4}$, whereas data on the accuracy of T1CRC detection at a community level are scarce. 
We aimed to determine the magnitude of the problem of missing T1CRCs in daily practice at community level. Therefore, we collected all Dutch

immunochemical test (FIT)-based BCSP colonoscopies in five representative, screening certified endoscopy units in the Southeast Netherlands between February 2014 and August 2015 and evaluated T1CRCs for correct optical diagnosis.

Prediction of histology was a mandatory query in the standardized BCSP-report. Endoscopists were certified for participation in the BCSP but did not receive additional training in optical diagnosis of T1CRC.

The main outcome was the accuracy for optical diagnosis of T1 CRC, where histological diagnosis is used as reference standard. Morphology and size were taken into account in a sub-analysis.

In addition, treatment consequences were evaluated, where a distinction was made between low-risk T1CRC (defined as the absence of lymphovascular invasion, poor differentiation and (R1-) resection margin $<1 \mathrm{~mm}$ ) and high-risk T1CRC (defined as presence of at least one of these features). The number of salvage surgeries (surgery after endoscopic resection) was reported.

A total of 115 T1CRCs were diagnosed in 2845 BCSP colonoscopies. Out of 115 diagnosed T1CRCs, only 24 were initially recognized as being malignant. The overall sensitivity for optical T1CRC recognition at community level was therefore $20.9 \%$. A sub-analysis showed that non-pedunculated T1CRCs were more easily recognized than pedunculated T1CRCs (25.8\% vs. $7.0 \%)$. Optical recognition of T1CRCs did not differ according to size (Table 1). 
For treatment consequences, the 91 initially non-recognized T1 CRCs were categorized into low-risk T1CRCs (defined as the absence of lymphovascular invasion, poor differentiation and (R1-)resection margin <1mm) and high-risk (defined as presence of at least one of three features). In the salvage surgery cases, 30 (33.0\%) were high-risk T1CRCs, of which 19 (63.3\%) showed only R1-resection margins as histological risk factor (Figure 1). There was no significant difference in curative (R0) endoscopic resection between pedunculated and non-pedunculated T1 CRCs (57.5\% vs. 46.3\%; $p=0.315$ ).

Incorrect optical diagnosis thus results in non-curative endoscopic resection in both pedunculated and non-pedunculated T1CRCs. This often leads to salvage surgery, which in a substantial number of cases appears to be unnecessary.

When interpreting our data, some shortcomings need to be acknowledged.

First, assessment of colorectal lesions and use of image-enhancement was not standardized and the level of confidence of optical diagnosis was not assessed.

Second, we included both pedunculated and non-pedunculated T1CRCs. For pedunculated T1CRCs the depth of submucosal invasion is more difficult to assess while for non-pedunculated lesions the increase in surface and vascular pattern disruption reflects the depth of submucosal invasion. ${ }^{5}$ This is supported by the lower sensitivity for pedunculated T1CRCs in our study.

Table 1. Optical diagnosis of T1CRCs of different morphology and size*

\begin{tabular}{|l|l|l|l|l|}
\hline & $\begin{array}{l}\text { Optically } \\
\text { diagnosed }\end{array}$ & $\begin{array}{l}\text { Total amount } \\
\text { of T1CRCs }\end{array}$ & Sensitivity & Sign. \\
\hline Pedunculated & 3 & 43 & $7.0 \%$ & $p=0.013$ \\
\hline Non-pedunculated & 17 & 66 & $25.8 \%$ & \\
\hline \multicolumn{7}{|l|}{} \\
\cline { 1 - 4 } Size 0-9mm & 0 & 4 & $0 \%$ & \multirow{2}{*}{ p=0.389 } \\
\hline Size $10-20 \mathrm{~mm}$ & 11 & 66 & $16.7 \%$ & \\
\hline Size $>20 \mathrm{~mm}$ & 8 & 38 & $21.1 \%$ & \\
\hline
\end{tabular}

*In 6 cases morphology was not reported and in 11 cases exact size was not reported. 
Figure 1. Flowchart of optical diagnosis and treatment of T1CRCs in BCSP.

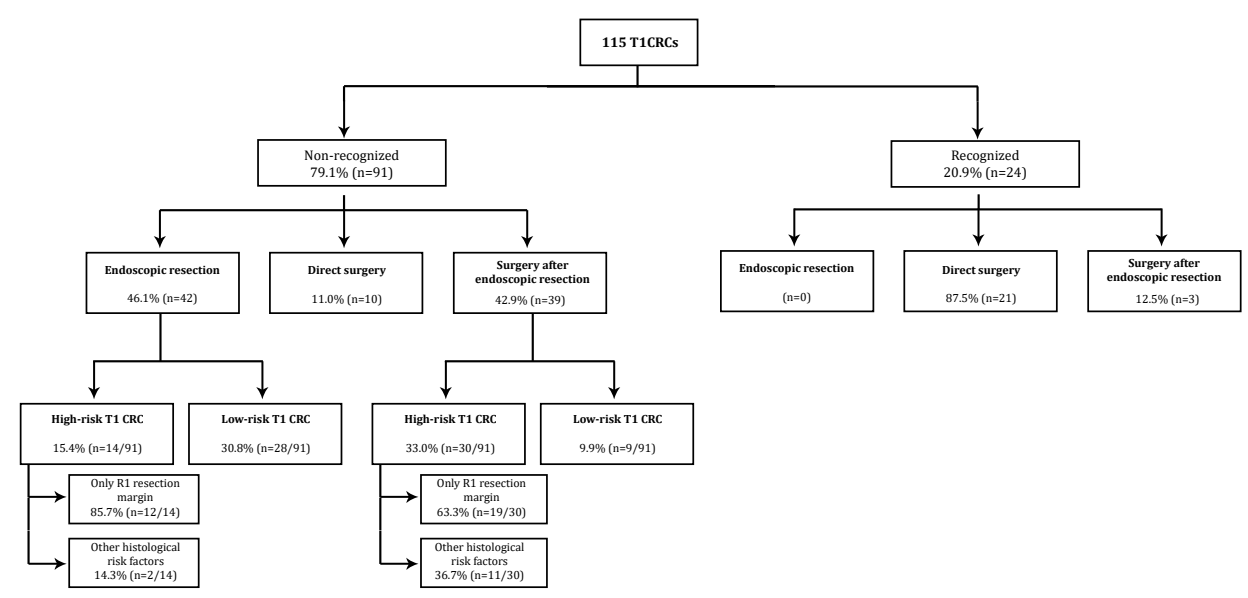

Endoscopic resection: only $R 1$ resection margin = (R1-), resection margin $<1 \mathrm{~mm}$; other histological risk, factors=lymphovascular invasion and poor differentiation.

To conclude, our study showed a poor sensitivity of only $20.9 \%$ for T1CRC optical diagnosis in daily clinical practice at community level, where most of the screening colonoscopies are being performed. Poor recognition of T1CRCs leads to noncurative endoscopic resection, which might lead to unnecessary additional surgical treatment in low-risk cases.

Improvement in recognition of T1CRCs is urgently needed. Implementation of the validated OPTICAL risk-chart in daily practice provides guidance by estimating CRCrisk. It should be considered as standardised component of the BCSP-training and BCSP-accreditation. 


\section{References}

1. Toes-Zoutendijk E, et al. Stage distribution of screen-detected colorectal cancers in the Netherlands. Gut 2018;67(9):1745-6

2. Backes $Y$, et al. Multicentre prospective evaluation of real-time optical diagnosis of $\mathrm{T} 1$ colorectal cancer in large non-pedunculated colorectal polyps using narrow band imaging (the OPTICAL study). Gut 2019;68(2):271-9.

3. Bronzwaer MES, et al. The occurrence and characteristics of endoscopically unexpected malignant degeneration in large rectal adenomas. Gastrointest Endosc 2018;87(3):862-71 e1

4. Burgess NG, et al. Risk Stratification for Covert Invasive Cancer Among Patients Referred for Colonic Endoscopic Mucosal Resection: A Large Multicenter Cohort. Gastroenterology 2017;153(3):73242 e1.

5. Puig I, et al. Accuracy of the Narrow-Band Imaging International Colorectal Endoscopic Classification System in Identification of Deep Invasion in Colorectal Polyps. Gastroenterology 2019;156(1):7587. 
T1 RECOGNITION AND TREATMENT 



\section{CHAPTER 6}

\section{Multitarget stool DNA testing and Faecal Immunochemical Testing (FIT) as alternative for colonoscopy-based surveillance: an interim analysis}

M.C.J. van Lanschot, A.J.P. van de Wetering, G.M. Lemmens, R.W.M. van der Hulst, A.M. van Berkel, A.C.T.M. Depla, M.E. van Leerdam, J.M. Jansen, C.A. Wientjes, J.W.A. Straathof, E.T.P. Keulen, D. Ramsoekh, B.M. Berger, G.P. Lidgard, M. Zacherl, S. Sanduleanu-Dascalescu, V.M.H. Coupé, A.A.M. Masclee, M. van Engeland, B. Carvalho, E. Dekker, G.A. Meijer 


\section{Abstract}

\section{Introduction}

The current colonoscopy-based surveillance programme for early detection of colorectal cancer (CRC) is a major burden for the healthcare system and likely not cost-effective. In the MOCCAS (MOlecular stool testing for Colorectal CAncer Surveillance) study, we aimed to assess whether the multitarget stool DNA test (Cologuard) could be used to preselect patients with advanced neoplasia (AN) for surveillance colonoscopy. Cologuard performance was compared to the faecal immunochemical test (FIT). Here we present an interim analysis.

\section{Methods}

In this cross-sectional study, whole stool samples for Cologuard testing and two FITs (OC Sensor and FOB Gold) were collected prior to their scheduled colonoscopy in post-CRC, post-polypectomy and familial risk surveillance patients. Sensitivity and specificity of Cologuard were calculated, applying the screening-validated threshold, and compared to FIT with a $15 \mu \mathrm{g} \mathrm{Hb} / \mathrm{g}$ faeces threshold for test positivity. Test performances were also compared at equal positivity rate of $50 \%$, translating into a reduction of surveillance colonoscopy volume by $50 \%$. Primary endpoint was AN detection as most advanced lesion. AN included CRC, advanced adenomas (AA) and advanced serrated polyps (ASP).

\section{Results}

Cologuard results were available for 1,551 patients, of which the majority (61\%) underwent post-polypectomy surveillance. AN was detected in 180 (11.6\%) patients, including 13 (0.8\%) CRC, 112 (7.2\%) AA and 55 (3.6\%) ASP. Cologuard detected AN with a sensitivity of $59 \%$ (95\% CI $52 \%$ to $67 \%$ ) and specificity of $76 \%$ (95\% CI $74 \%$ to $79 \%$ ). Sensitivity for CRC was $77 \%$ (95\% CI $46 \%$ to $95 \%)$, for AA $51 \%$ (95\% CI $42 \%$ to $61 \%$ ) and for ASP $71 \%$ (95\% CI $57 \%$ to $82 \%)$. Both FIT OC Sensor and FOB Gold had lower sensitivity for AN (27\% (95\% CI 20\% to 34\%) and 34\% (95\% CI $27 \%$ to $41 \%$ ), respectively, $p<0.001)$ and higher specificity $(89 \%$ (95\% CI $97 \%$ to $91 \%$ ) and $86 \%$ (95\% CI 84\% to 88\%), respectively, $p<0.001$ ) than Cologuard. At $50 \%$ positivity rate, Cologuard sensitivity for AN was $76 \%(95 \% \mathrm{CI} 69 \%$ to $82 \%$ ) and specificity 56\% (95\% CI 53\% to 58\%). At this threshold, Cologuard test sensitivity for AN remained significantly higher than both FITs (OC Sensor 63\% (95\% CI 55\% to $70 \%$ ) and FOB Gold 57\% (95\% CI 49\% to 65\%), $p<0.01$ ), while specificities were comparable.

\section{Conclusion}

The multitarget stool DNA test (Cologuard) has higher sensitivity than FIT for detection of AN in the surveillance population. Applying a low test cut-off to reach $50 \%$ positivity rate would further increase Cologuard sensitivity and still reduce the number of unnecessary colonoscopies by half. Mathematical modelling approaches will be applied to the final study data in order to determine an optimal stool-based strategy for surveillance. 


\section{Introduction}

Screening programmes have been implemented in many countries to reduce colorectal cancer (CRC) incidence and mortality. Independently of the screening method used, this leads to more patients being diagnosed with CRC and polyps. Observational studies have shown that patients having undergone curative CRC resection or polypectomy are at increased risk for CRC compared to the general population. ${ }^{1-3}$ Therefore, this population is recommended to undergo regular surveillance colonoscopies, reason why screening programs indirectly also lead to an increase in surveillance.

Similar to screening, the goal of a surveillance programme is to prevent CRC and death from CRC by early detection of (pre)malignant lesions. However, the benefit of the current colonoscopy-based surveillance strategies remains unclear. ${ }^{4}$ Recommendations are based on older cohorts, ${ }^{5,6}$ but since then substantial improvements in colonoscopy quality have been made. This has reduced the number of missed or incompletely removed lesions. ${ }^{7}$ Only few studies have investigated the effect of surveillance on long-term CRC incidence, instead of the effect on incidence of advanced adenomas (AA). ${ }^{6,8}$ A recent study showed that a proportion of patients that are advised surveillance colonoscopy every three years might not benefit from this. ${ }^{9}$

Because of limited long-term follow-up data, a microsimulation model was used to examine the added value of colonoscopy surveillance in a screening setting. ${ }^{4}$ They found that colonoscopy based surveillance on top of a FIT based screening program reduced CRC mortality by an extra $1.7 \%$ (from $50.4 \%$ to $52.1 \%$ ), whilst increasing the lifetime colonoscopy demand by $62 \%$. Thus, in this modelling study, adding colonoscopy surveillance to FIT screening was not cost-effective. ${ }^{4}$

Taken together, the current colonoscopy-based surveillance strategy likely leads to overdiagnosis. Patients are exposed to unnecessary harm and the limited colonoscopy capacity is used inefficiently. In addition, colonoscopy is expensive and associated with a low risk of bleeding and perforation. ${ }^{10}$ 
Currently, 20-25\% of colonoscopies are performed for post-polypectomy surveillance. ${ }^{11,12}$ Without changing policies, this number will gradually increase further due to the implementation of population screening programs and the performance of modern, high-quality endoscopy. Colonoscopy has been the primary method for surveillance since the early $1980 \mathrm{~s}^{13}$, but comparative studies with other modalities are scarce. With the availability of new stool tests, the question has become relevant to study whether these tests could be used for surveillance and potentially postpone colonoscopy safely.

The faecal immunochemical test (FIT), produced by multiple manufacturers, detects human haemoglobin $(\mathrm{Hb})$ in faeces. FIT has proven to be an effective method for early CRC detection and is widely used for screening. ${ }^{14} \mathrm{~A}$ recent surveillance study with three FIT rounds showed that annual testing was a cost-saving strategy in the intermediate risk population. ${ }^{15}$ Yet, sensitivity for AN of a single FIT round at low threshold in the surveillance setting was $20-34 \%$ at specificity of $88-92 \%{ }^{15}$ The multitarget stool DNA test (Cologuard, Exact Sciences, Madison, WI, USA) combines the detection of human $\mathrm{Hb}$ with several DNA markers and has only be tested in the screening setting. In the screening population, Cologuard detected advanced neoplasia (AN) with a sensitivity of $46 \%$ at a specificity of $87 \% .{ }^{16}$

In the MOCCAS (MOlecular stool testing for Colorectal CAncer Surveillance) study, we hypothesized that regular stool testing could be used to preselect patients for surveillance colonoscopies. The primary aim of this study was to assess whether the multitarget stool DNA test (Cologuard) could accurately patients with advanced neoplasia (AN) for surveillance colonoscopy. Secondly, we compared the performance of Cologuard to FIT. Ultimately, these data will be used as input for cost-effectiveness modelling to find the optimal stool-based surveillance strategy. Here, we present an interim analysis of the MOCCAS study. 


\section{Methods}

\section{Study design}

This interim analysis is part of the MOlecular stool testing for Colorectal CAncer Surveillance (MOCCAS) study, a prospective, multicentre, cross-sectional study ongoing in eleven endoscopy centres throughout the Netherlands. For the interim analysis, patients were enrolled between November 2015 and April 2018. The study was approved by the Medical Ethical Committee of the Amsterdam UMC, the Netherlands and registered in ClinicalTrials.gov (NCT02715141). Details on the study protocol can be found elsewhere. ${ }^{17}$

\section{Study population}

Patients between 50-75 years old, with an indication for surveillance colonoscopy according to the Dutch surveillance guidelines (i.e. post-polypectomy, history of CRC or familial risk) visiting any of the participating centres were eligible. ${ }^{18}$ In case of inflammatory bowel disease (IBD), genetic cancer syndromes (e.g. Lynch syndrome and familial adenomatous polyposis), colonoscopy in the previous 6 months, proctocolectomy or life expectancy less than three years, were excluded. Patients had to have sufficient understanding of the Dutch language in order to provide written informed consent.

\section{Enrolment procedures}

Patients with a scheduled routine surveillance colonoscopy were invited by a member of the research team to participate two weeks prior to their colonoscopy. After the patient's consent, the test kit containing instructions and stool tests (Cologuard and two different FITs) were shipped to the patients' home address. Patients performed a whole stool specimen and FIT collection from the same bowel movement at home before starting bowel preparation. Before colonoscopy examination, patients returned the kit at the endoscopy department. Within 72 hours after collection, stool samples were transferred from the endoscopy department to the laboratory site and processed. 
CHAPTER 6

\section{Colonoscopy examination}

Colonoscopy was performed according to standard practice. The endoscopists documented caecal intubation status and bowel preparation score as colonoscopy quality parameters and also location, size and morphology of any lesions detected. Bowel preparation of $\geq 6$ on the Boston Bowel Preparation Scale was considered good and $<6$ poor. ${ }^{19}$ Lesions removed were collected for histological evaluation. Endoscopists and pathologists were blinded for the results of the stool tests. Patient data were extracted from endoscopy data systems, the Dutch national pathology archive PALGA and hospital electronic health records.

Patients were not informed about the faeces study test results, as they already underwent a colonoscopy.

The collection of clinical data was performed according to the Dutch Personal Data Protection Act, using a predefined CRF with a dedicated electronic data capture system validated to global regulatory standards (i.e. OpenClinica, Waltham, MA, USA).

\section{Laboratory procedures}

Stool specimens were shipped to either one of the two central laboratories in the Netherlands. For Cologuard, buffered stool samples were homogenised, aliquoted and frozen at $-80^{\circ} \mathrm{C}$ in compliance standard operating procedures. Stool aliquots were shipped in batches to Exact Sciences, where analyses were performed. Cologuard consists of an immunochemical assay for human $\mathrm{Hb}$ and molecular assays for mutations in codon 12 of the KRAS gene (referred to as KRAS1) and in codon 13 of the KRAS gene (referred to as KRAS2), and hypermethylation of NDRG4 and $B M P 3$ promoter regions. Also, $\beta$-actin is part of this molecular assay, acting as reference gene for human DNA quantity. Quantitative measurements for each marker separately were obtained, as well as a single result for the multitarget stool DNA test. For $\mathrm{Hb}$, the maximum value was $60 \mu \mathrm{g} \mathrm{Hb} / \mathrm{g}$ faeces (i.e. $600 \mathrm{ng} \mathrm{Hb} / \mathrm{ml}$ buffer). Calling of an individual test as positive or negative was done using the screening-validated threshold of $182 .{ }^{16}$ 
The two FITs used in this study were OC Sensor (Eiken Chemical Co, Tokyo, Japan) and FOB Gold (Sentinel, Milano, Italy). FIT samples were stored at $4^{\circ} \mathrm{C}$ upon arrival and measured according to the manufacturer's instruction by a trained technician, yielding quantitative measures of $\mathrm{Hb}$ concentration.

Pre-defined thresholds were applied, which were used in previous screening trials in the Netherlands. ${ }^{20}$ For both the OC Sensor and FOB Gold, this threshold was $15 \mu \mathrm{g}$ $\mathrm{Hb} / \mathrm{g}$ faeces (i.e. $75 \mathrm{ng} \mathrm{Hb} / \mathrm{ml}$ buffer and $88 \mathrm{ng} \mathrm{Hb} / \mathrm{ml}$ buffer, respectively). ${ }^{21} \mathrm{To}$ compare the $\mathrm{Hb}$ values between the three tests (see statistical analyses), a maximum value of $60 \mu \mathrm{g} \mathrm{Hb} / \mathrm{g}$ faeces was applied for the FITs in the same way as for Cologuard. Measurements of all tests were performed blinded for the colonoscopy results.

\section{Outcome measures}

Primary outcome measures were sensitivity and specificity of Cologuard for AN. AN included CRC, AA (adenoma $\geq 10 \mathrm{~mm}$ and/or with high grade dysplasia and/or $\geq 25 \%$ villous component), and advanced serrated polyps (ASP; hyperplastic polyp/ sessile serrated lesion/traditional serrated adenoma $\geq 10 \mathrm{~mm}$ or with dysplasia) as most advanced lesion. Secondary outcomes were sensitivity and specificity of the two FITs. In addition, Hb concentrations and DNA marker values were used for comparison between different lesion types.

\section{Sample size calculation}

The test characteristics of Cologuard were used to calculate the required sample size. A similar test sensitivity of $50 \%$ for $\mathrm{AN}$, as was previously reported for a large screening population, was assumed for the surveillance population. ${ }^{16}$ In order to obtain an accuracy of $5 \%$ around the sensitivity estimate, a total of 400 individuals with AN was needed. In a recent Dutch surveillance study AA were detected in one per ten individuals. ${ }^{22}$ Based on this ratio, a sample size of 4,000 individuals was needed to allow an accurate estimate of the specificity of Cologuard in this population $(2 \%$ estimated width of $95 \%$ confidence interval (CI)). This interim analysis presents the results of a subset of 1,756 cases. 


\section{Statistical analysis}

For Cologuard as well as the respective FITs, sensitivities were calculated using the pre-specified screening thresholds for test positivity. In addition, sensitivities were compared using equal positivity rate of $50 \%$, which would translate into a reduction of surveillance colonoscopy volume by $50 \%$. Colonoscopy results combined with histopathology results were considered the reference standard. Calculation of the specificity was done considering all patients with non-advanced adenomas, nonadvanced serrated lesions, non-neoplastic findings or a negative colonoscopy as controls. In addition, specificity was calculated considering only patients with a complete negative colonoscopy as controls. For determining the $95 \% \mathrm{CI}$ around the test characteristics, the exact binomial test was applied using the R package epiR. The McNemar test was used to compare differences in lesion detection between the respective diagnostic tests.

Receiver Operating Characteristic (ROC) curves were constructed in order to calculate the ability of the tests to discriminate between patients with and without the disease (i.e. CRC or AN versus non-advanced neoplasia or lesser findings) for different thresholds. The discriminatory ability was summarised in the area under the curve (AUC) and DeLong's method was used to test the differences in AUC between tests. For these calculations, the R package pROC was used.

To compare $\mathrm{Hb}$ value of Cologuard and the FITs for CRC, AA and ASP separately, the Wilcoxon rank sum test was used because of the non-normal distribution of the marker values.

Subgroup analyses were performed based on the most severe surveillance indication. Assuming patients with a history of CRC have the highest risk for metachronous CRC, followed by patients with a polypectomy and then by patients with a familial risk, three groups were distinguished: 1) post-CRC surveillance population, 2) post-polypectomy surveillance population, without history of CRC, 3) familial risk surveillance population, without history of CRC or post-polypectomy indication. To calculate differences in lesion distribution among surveillance indication subgroups, the Fisher's Exact test was used. All analyses were conducted in R studio, version 1.1.383 (R studio, Boston, MA, USA). 


\section{Results}

\section{Study population}

A total of 1,756 patients were enrolled in the study at the time of data-extraction on April 12th 2018. Of these, 1,551 patients had Cologuard results completed. Reasons for drop-out are listed in the flowchart in Figure 1.

Of the 1,551 patients that were included in the primary analysis, 54\% were male and mean age was 66 years (SD 6.4) (Table 1). The most common indication for colonoscopy was post-polypectomy surveillance (61\%), followed by post-CRC surveillance (33\%) and familial risk for CRC (6\%) (Table 1). The caecum was reached in $96 \%$ and bowel preparation was good in $85 \%$. At the surveillance colonoscopy, AN was detected in $11.6 \%$, concerning $0.8 \%$ CRC, $7.2 \%$ AA and 3.6\% ASP.

Table 1. Baseline characteristics

\begin{tabular}{|c|c|c|}
\hline \multicolumn{2}{|l|}{ Total cohort } & $n=1,551$ \\
\hline \multicolumn{3}{|l|}{ Patient demographics } \\
\hline Male, n (\%) & & $839(54)$ \\
\hline Age, years (SD) & & $66(6.4)$ \\
\hline \multirow[t]{3}{*}{ Surveillance indication, $\mathrm{n}(\%)$} & Post-CRC & $507(33)$ \\
\hline & Post-polypectomy & $942(61)$ \\
\hline & Familial risk & $102(66)$ \\
\hline \multicolumn{3}{|l|}{ Quality of colonoscopy } \\
\hline \multirow[t]{3}{*}{ Caecum reached, $\mathrm{n}(\%)$} & Yes & $1,486(95.8)$ \\
\hline & No & $4(0.3)$ \\
\hline & Not reported & $61(3.9)$ \\
\hline \multirow[t]{3}{*}{ Bowel preparation, $\mathrm{n}(\%)$} & Good & $1,314(84.7)$ \\
\hline & Poor & $2(0.1)$ \\
\hline & Not reported & $235(15.2)$ \\
\hline \multicolumn{3}{|c|}{ Findings at surveillance (most advanced lesion) } \\
\hline \multirow[t]{4}{*}{ Advanced neoplasia, n (\%) } & Colorectal cancers & $13(0.8)$ \\
\hline & Advanced adenomas & $112(7.2)$ \\
\hline & Advanced serrated polyps & $55(3.6)$ \\
\hline & Total & $180(11.6)$ \\
\hline
\end{tabular}




\section{CHAPTER 6}

Figure 1. Flowchart of interim study population

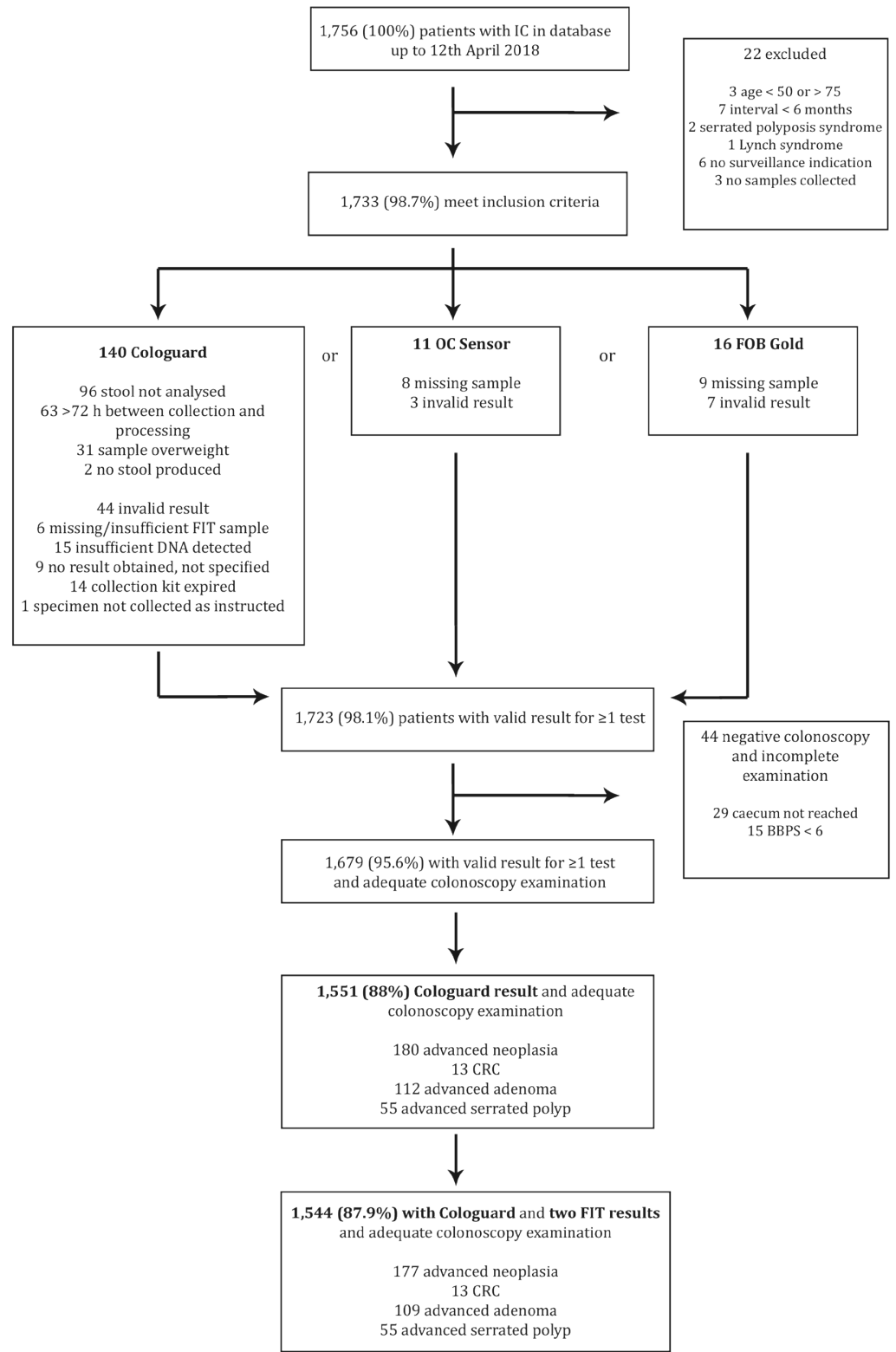

BBPS: Boston Bowel Preparation Score, CRC: colorectal cancer 


\section{Cologuard performance}

Cologuard detected 106 of 180 patients with AN, corresponding to a sensitivity of Cologuard of 59\% (95\% CI 51\% to 66\%) (Table 2). Out of the thirteen patients with CRC, Cologuard detected ten (77\%, 95\% CI 46\% to 95.0\%). Sensitivity for detecting AA was $51 \%$ (57 of $112,95 \%$ CI $41 \%$ to 61 ) \% and for ASP $71 \%$ (39 of $55,95 \%$ CI $57 \%$ to $82 \%)$.

Among 1,371 patients who had findings other than AN or negative colonoscopy, the Cologuard identified 1,045 patients correctly as negative (specificity $76 \%, 95 \% \mathrm{CI}$ $74 \%$ to $79 \%$ ). For those patients with a totally negative colonoscopy, specificity was $81 \%$ (483 of $600,95 \%$ CI $77 \%$ to $84 \%$ ).

Table 2. Test characteristics of Cologuard at screening threshold

\begin{tabular}{|l|l|l|l|}
\hline $\begin{array}{l}\text { Most advanced lesion } \\
\text { (n=1,551) }\end{array}$ & Colonoscopy & $\begin{array}{l}\text { Cologuard } \\
\text { (score 182) }\end{array}$ & \\
\hline & & $\begin{array}{l}\text { + result } \\
(\mathrm{n}=432)\end{array}$ & Sensitivity \% (95\% CI) \\
\hline Advanced neoplasia & 180 & 106 & 59 (51 to 66) \\
\hline Colorectal cancers & 13 & 10 & $77(46$ to 95) \\
\hline Advanced adenomas & 112 & 57 & $51(41$ to 61) \\
\hline Advanced serrated polyps & 55 & 39 & $71(57$ to 82) \\
\hline Non-advanced neoplasia & 600 & 172 & $29(25$ to 33) \\
\hline & & & \\
\hline & & - result & Specificity \% (95\% CI) \\
\hline \begin{tabular}{l|l|l|l|} 
Non-advanced lesions, other \\
lesions and negative colonoscopy
\end{tabular} & 1,371 & 1,045 & 76 (74 to 79) \\
\hline Negative colonoscopy & 600 & 483 & 81 (77 to 84) \\
\hline
\end{tabular}

\section{Comparison of Cologuard to FIT}

Test performance at screening threshold

For the comparison of Cologuard to FIT, participants with test results for Cologuard as well as the two FITs were used, yielding 1,544 patients. The performance of Cologuard in these individuals was similar to that of the entire group of study participants (Table 3). OC Sensor had a sensitivity for AN of $27 \%$ (95\% CI $20 \%$ to 34\%) (Table 3). 
CHAPTER 6

Table 3. Test characteristics of Cologuard and FIT at screening threshold

\begin{tabular}{|c|c|c|c|c|c|c|c|c|c|c|}
\hline & 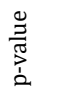 & $\begin{array}{l}\vec{\Delta} \\
\dot{0} \\
\mathrm{i}\end{array}$ & $\begin{array}{l}\text { L } \\
\text { O̦ }\end{array}$ & $\begin{array}{l}+ \\
\dot{0} \\
0\end{array}$ & $\begin{array}{l}\vec{\circ} \\
\dot{0} \\
\text { v }\end{array}$ & $\begin{array}{l}\vec{\Delta} \\
\dot{0} \\
\mathrm{v}\end{array}$ & & $\begin{array}{l}\vec{\Xi} \\
\dot{0} \\
\dot{0}\end{array}$ & $\begin{array}{l}\overrightarrow{8} \\
\dot{8} \\
\dot{v}\end{array}$ & \\
\hline & 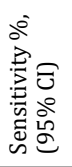 & 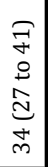 & 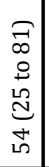 & 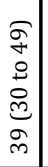 & 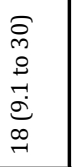 & 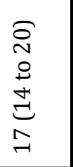 & 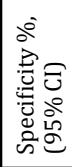 & $\begin{array}{r}\infty \\
\infty \\
\infty \\
\stackrel{+}{+} \\
\infty\end{array}$ & 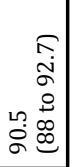 & \\
\hline \multirow[t]{3}{*}{ 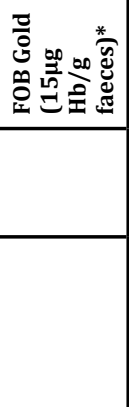 } & 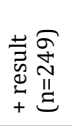 & 8 & $\wedge$ & $\mathscr{F}$ & 우 & $\underset{\sim}{\stackrel{N}{\sigma}}$ & 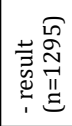 & $\begin{array}{l}\stackrel{\infty}{\stackrel{-}{-}} \\
=\end{array}$ & 音 & \\
\hline & 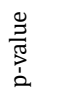 & $\begin{array}{l}\vec{\Delta} \\
\dot{0} \\
\dot{v}\end{array}$ & $\begin{array}{l}\mathcal{N} \\
\stackrel{0}{0}\end{array}$ & $\begin{array}{l}\overrightarrow{0} \\
\dot{0} \\
\dot{0} \\
v\end{array}$ & $\begin{array}{l}\overrightarrow{8} \\
\dot{0} \\
\dot{v}\end{array}$ & $\begin{array}{l}\tilde{\Delta} \\
\dot{0} \\
\dot{v}\end{array}$ & & $\begin{array}{l}\vec{\Delta} \\
\dot{0} \\
\dot{v}\end{array}$ & $\begin{array}{l}-1 \\
\dot{8} \\
\dot{0} \\
\mathrm{v}\end{array}$ & \\
\hline & 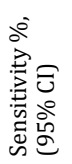 & 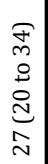 & 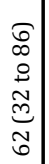 & 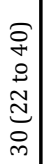 & 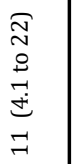 & 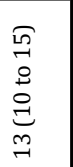 & 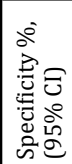 & 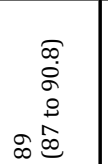 & 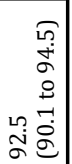 & \\
\hline \multirow[t]{2}{*}{ 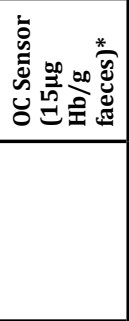 } & 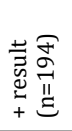 & f & $\infty$ & $m$ & 0 & $\stackrel{+}{\curvearrowright}$ & 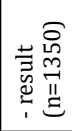 & $\underset{\sim}{\stackrel{N}{N}}$ & 员 & 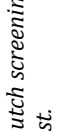 \\
\hline & 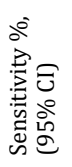 & 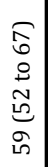 & 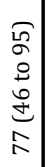 & 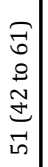 & 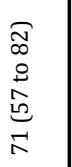 & 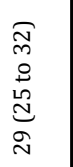 & 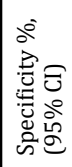 & 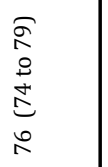 & 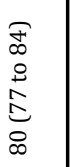 & 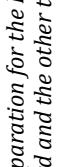 \\
\hline 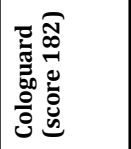 & 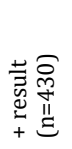 & $\stackrel{\text { L }}{\sim}$ & $\circ$ & 니 & बे & 곡 & 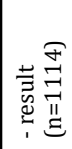 & 辛 & $\stackrel{\vec{\infty}}{+}$ & 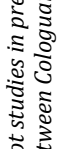 \\
\hline 产 & & $\stackrel{尺}{\therefore}$ & $m$ & $\stackrel{\circ}{\circ}$ & 능 & $\begin{array}{l}\infty \\
\text { ô } \\
\text { in }\end{array}$ & & $\begin{array}{l}\hat{\sigma} \\
m \\
\sim\end{array}$ & $\begin{array}{l}\infty \\
\text { ஸे }\end{array}$ & 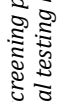 \\
\hline 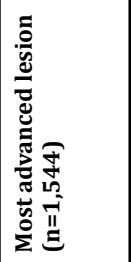 & & 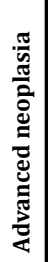 & 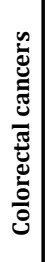 & 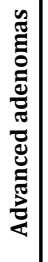 & 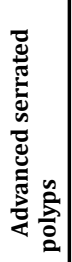 & 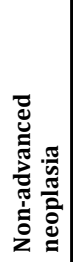 & & 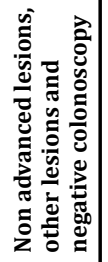 & 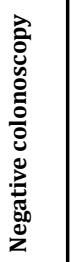 & 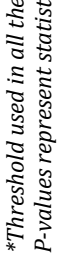 \\
\hline
\end{tabular}


Sensitivity for CRC was $62 \%$ (95\% CI 32\% to 86\%), for AA 30\% (95\% CI $22 \%$ to $40 \%$ ) and for ASP $11 \%$ (95\% CI $4.1 \%$ to $22 \%$ ).

Compared to Cologuard, the sensitivity for detection of AN, AA and ASP was significantly lower for OC Sensor $(p<0.001)$. Sensitivity for CRC did not differ significantly between Cologuard and OC Sensor.

Specificity of OC Sensor for AN was 89\% (95\% CI 87\% to 90.8\%). Specificity was 92.5\% (95\% CI 90.1\%-94.5\%) considering patients with fully negative colonoscopy as controls. This was significantly higher than the specificity of Cologuard for these two groups $(p<0.001)$.

FOB Gold had a sensitivity for AN of 34\% (95\% CI 27\% to 41\%). The sensitivity for CRC was 54\% (95\% CI 25\% to 81\%), for AA 39\% (95\% CI 30\% to 49\%) and for ASP 18\% (95\% CI 9.1\% to 30\%). Sensitivity for detection of AN, AA and ASP was significantly lower for FOB Gold than for Cologuard $(p<0.05)$. Also here, sensitivity for CRC did not differ significantly between Cologuard and FOB Gold.

Specificity of FOB Gold for AN was 86\% (95\% CI 84\% to 88\%). Specificity was 90.5\% (95\% CI 88\% to $92.7 \%$ ) considering patients with totally negative colonoscopy as controls. In both cases specificity was significantly higher compared to Cologuard $(p<0.001)$.

\section{Discriminatory ability}

For discriminating between patients with and without AN, the AUC was 0.73 for Cologuard. This was significantly higher than the AUC for OC Sensor of $0.64(p<0.001)$ and the AUC for FOB Gold of $0.61(p<0.001$ ) (Figure $2 A)$. The AUC for CRC was 0.80 for Cologuard, compared to 0.87 and 0.86 for FIT (Figure 2B). These differences in AUC were not significant. In Supplementary Figures $1 B$ and $1 D$, ROC curves are shown considering patients with negative colonoscopy only as the control group. 


\section{CHAPTER 6}

Figure 2. ROC curve comparing Cologuard with FIT for advanced neoplasia and colorectal cancer detection

A. Advanced neoplasia

Test - a. Cologuard - b. OC Sensor - c. FOB Gold

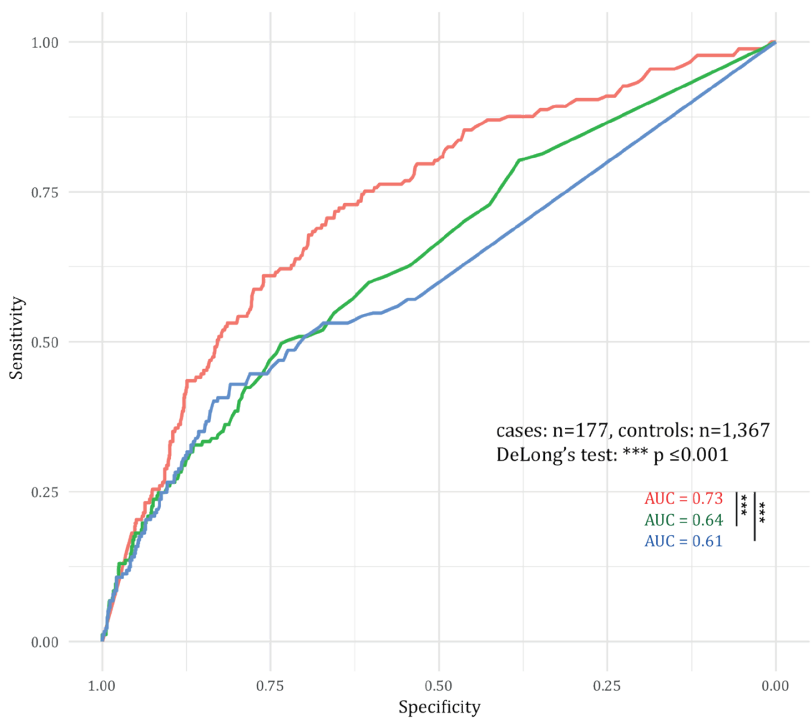

B. Colorectal cancer

Test - a. Cologuard - b. OC Sensor - c. FOB Gold

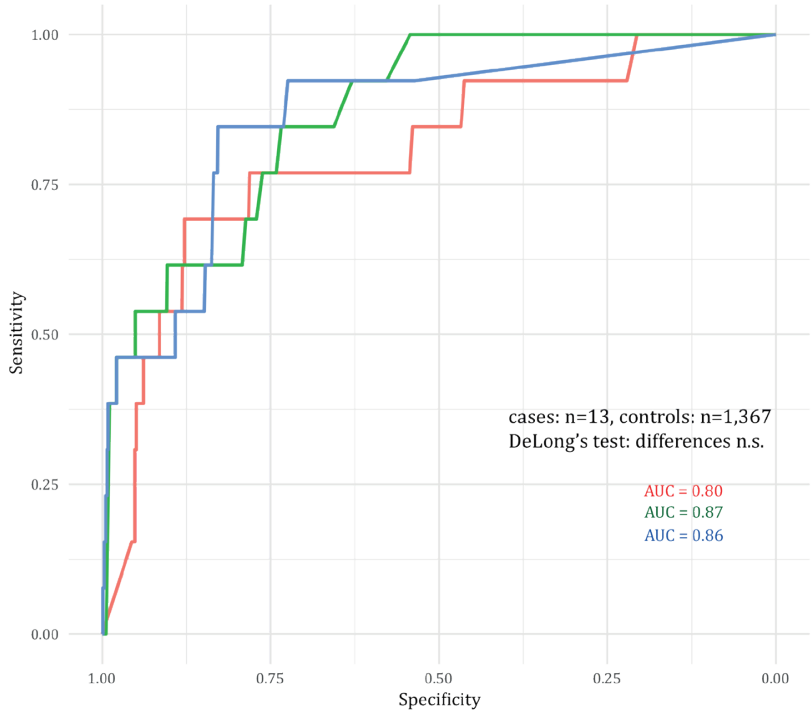




\section{Test performance at $50 \%$ positivity rate}

Cologuard was compared to FIT at equal positivity rate of $50 \%$. When FOB Gold threshold was lowered to $0 \mu \mathrm{g} \mathrm{Hb} / \mathrm{g}$ faeces (i.e. when all patients with FOB Gold values $>0 \mu \mathrm{g} \mathrm{Hb} / \mathrm{g}$ faeces were considered positive and all patients with values equal to zero were considered negative), test positivity rate was $48 \%$. Because the FOB Gold threshold could not be lowered beyond this value, all tests were compared at positivity rate of $48 \%$, equal to 736 referrals for colonoscopy.

At this positivity rate, Cologuard sensitivity for AN detection was 76\% (95\% CI $69 \%$ to $82 \%$ ) (Table 4). Cologuard sensitivity for CRC was 77\% (95\% CI $46 \%$ to 95.0\%), for AA 73\% (95\% CI 63\% to 81\%) and for ASP 84\% (95\% CI 71\% to $92.2 \%$ ). Cologuard specificity for AN was 56\% (95\% CI 53\% to 59\%).

Sensitivity for AN was significantly higher for Cologuard than for OC Sensor (63\%, $95 \%$ CI 55\% to 70\%) and FOB Gold (57\%, 95\% CI 49\% to 65\%) ( $p<0.01)$ (Table 4). Cologuard detected ASP with significantly higher sensitivity compared to OC Sensor (44\%, 95\% CI 30\% to 58\%) and FOB Gold (36\%, 95\% CI $24 \%$ to 50\%) $(p<0.001)$. The sensitivity of the three tests did not differ significantly for CRC and AA detection. Also specificities were similar for all three tests.

\section{Test markers in relation to lesion type}

In Figure $3 A \mathrm{Hb}$ values of the three tests are shown in relation to the most advanced lesion, i.e. CRC, AA and ASP. For all three tests, median Hb values were significantly lower in patients with AA compared to patients with CRC $(p<0.05)$. Hb values were also lower in patients with ASP compared to those with CRCs $(p<0.0001)$ and AA $(p<0.01)$ as most advanced lesion.

In Figure $3 B$ the molecular DNA markers KRAS1, KRAS2, BMP3 and NDRG4 of Cologuard are shown in relation to the most advanced lesion. The median values of the hypermethylation markers BMP3 and NDRG4 differed significantly across lesion types and was higher in patients with ASP than in those AA $(p<0.001)$. 
CHAPTER 6

Table 4. Test characteristics of Cologuard and FIT at equal specificity of $50 \%$.

\begin{tabular}{|c|c|c|c|c|c|c|c|c|c|c|}
\hline & 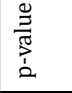 & $\begin{array}{l}\overrightarrow{8} \\
\dot{0} \\
\dot{v}\end{array}$ & $\begin{array}{l}\tilde{S} \\
0\end{array}$ & $\stackrel{+}{\circ}$ & ¿े & $\begin{array}{l}l_{0} \\
0 \\
0 \\
0\end{array}$ & & ָ̊ & กี & క్ర \\
\hline & 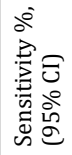 & $\begin{array}{l}\text { I } \\
0 \\
0 \\
0 \\
0 \\
\vdots \\
\text { in }\end{array}$ & 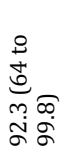 & 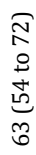 & 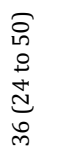 & 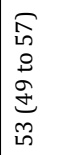 & 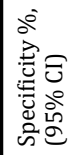 & 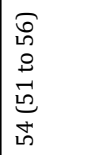 & $\begin{array}{l}\text { f } \\
0 \\
0 \\
0 \\
0 \\
0 \\
0\end{array}$ & 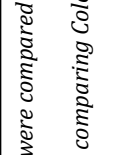 \\
\hline \multirow[t]{3}{*}{ 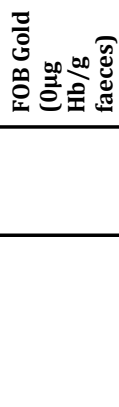 } & 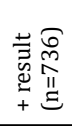 & $\stackrel{\vec{\sigma}}{-}$ & $\approx$ & o & iి & $\frac{n}{m}$ & 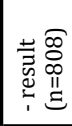 & $\tilde{\aleph}$ & $\overrightarrow{\mathrm{e}}$ & 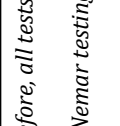 \\
\hline & \begin{tabular}{l}
0 \\
\multirow{3}{*}{} \\
2 \\
2 \\
2
\end{tabular} & $\begin{array}{l}\hat{0} \\
0 \\
0\end{array}$ & 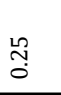 & 旁 & $\begin{array}{l}\vec{\circ} \\
\dot{0} \\
\dot{v}\end{array}$ & $\stackrel{N}{\hat{O}}$ & & 䇉 & $\stackrel{\text { fे }}{0}$ & 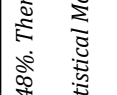 \\
\hline & 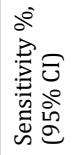 & $\begin{array}{l}2 \\
2 \\
0 \\
0 \\
0 \\
0 \\
0 \\
0\end{array}$ & 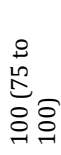 & $\begin{array}{l}\text { 尺 } \\
0 \\
0 \\
\infty \\
0 \\
0 \\
0 \\
0\end{array}$ & 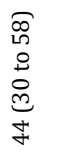 & 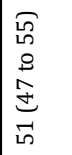 & 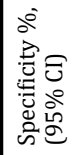 & 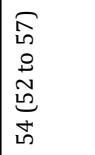 & $\begin{array}{l}\text { F } \\
0 \\
0 \\
0 \\
0 \\
.0 \\
8 \\
0\end{array}$ & 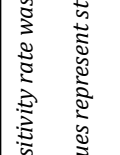 \\
\hline \multirow[t]{2}{*}{ 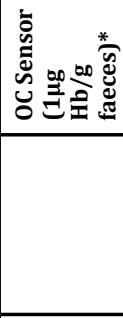 } & 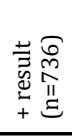 & $\exists$ & $\stackrel{m}{\rightarrow}$ & $\stackrel{+}{\sim}$ & $\stackrel{\mathbb{N}}{ }$ & वे & 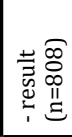 & $\vec{F}$ & : & 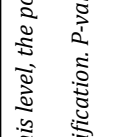 \\
\hline & 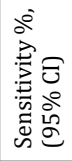 & $\begin{array}{l}\widetilde{1} \\
0 \\
0 \\
0 \\
0 \\
0 \\
0 \\
1\end{array}$ & 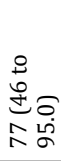 & $\begin{array}{l}\overparen{c} \\
\infty \\
0 \\
0 \\
0 \\
0 \\
m \\
n\end{array}$ & 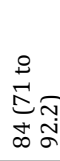 & 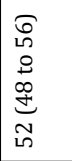 & 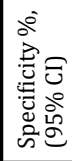 & 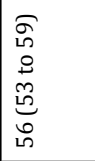 & 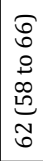 & 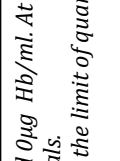 \\
\hline 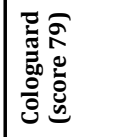 & 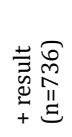 & 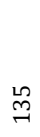 & 우 & $\stackrel{2}{1}$ & f & $\vec{m}$ & 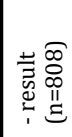 & ¿े & $\stackrel{N}{\stackrel{N}{m}}$ & 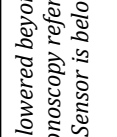 \\
\hline 产 & & 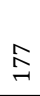 & $\stackrel{m}{\rightarrow}$ & 空 & 뉴ำ & $\begin{array}{l}\infty \\
\vdots \\
i n\end{array}$ & & $\begin{array}{l}\hat{0} \\
\text { m } \\
-\end{array}$ & $\begin{array}{l}\infty \\
\text { in } \\
\text { in }\end{array}$ & 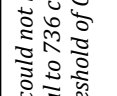 \\
\hline 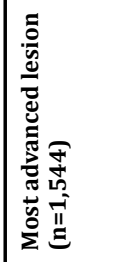 & & 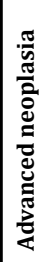 & 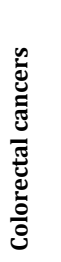 & 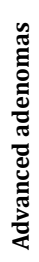 & 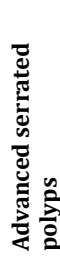 & 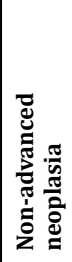 & & 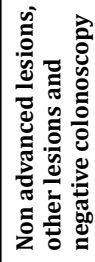 & 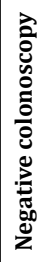 & 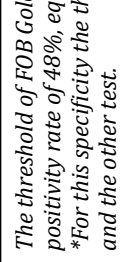 \\
\hline
\end{tabular}


Figure 3. Markers values versus lesion type A. Haemoglobin ( $\mathrm{Hb}$ ) values for Cologuard, OC Sensor and FOB Gold per lesion type B. Values of Cologuard DNA markers per lesion type

\section{A. Haemoglobine concentration}

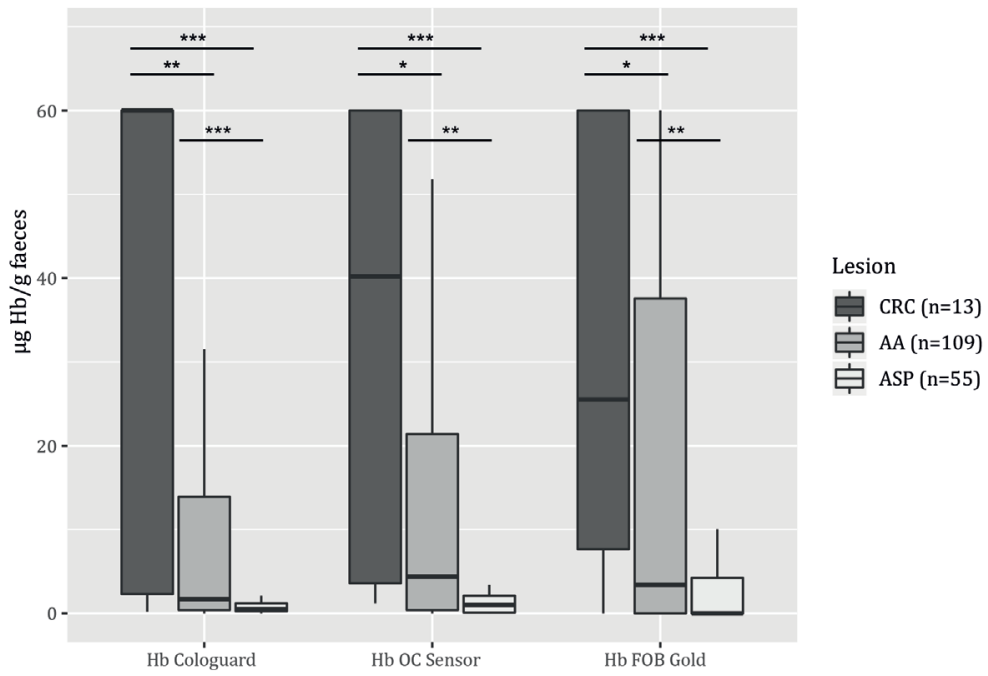

B. Cologuard Markers

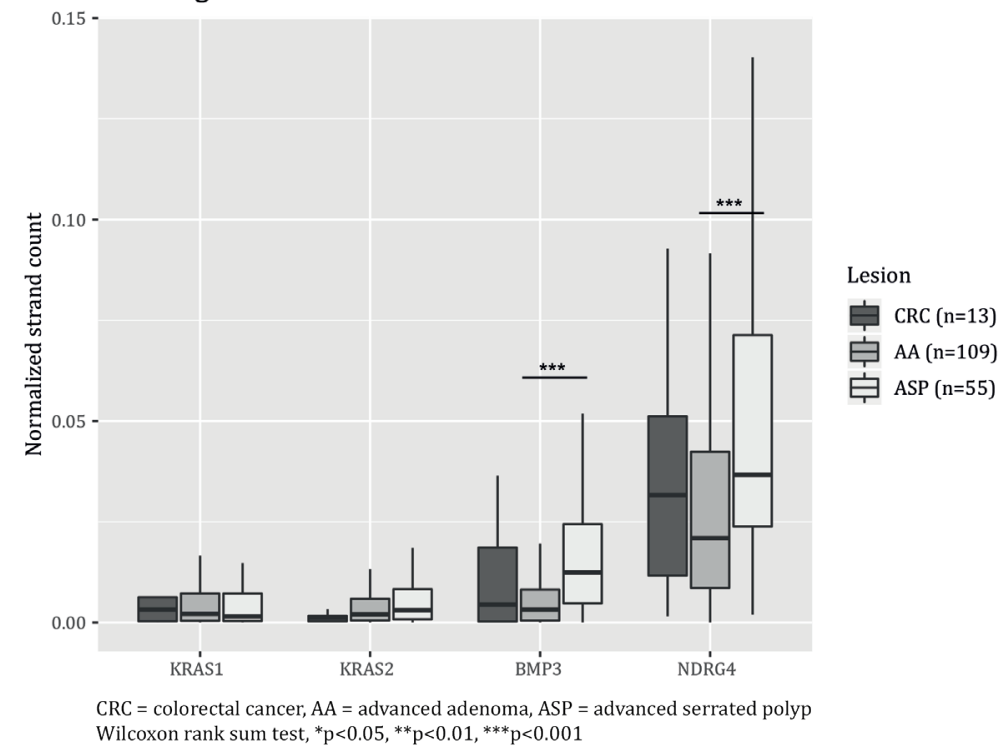




\section{Lesion distribution by surveillance-indication}

Most CRC (9/13) were found in patients undergoing post-CRC surveillance (Figure 4). This was reflected by a significantly higher risk of CRC at colonoscopy in the post-CRC surveillance population compared to the post-polypectomy surveillance population (OR 4.2 (95\% CI 1.2 to 19), $p<0.05$ ). In patients undergoing postpolypectomy surveillance, AA was more frequently detected compared to the postCRC population (OR 2.2 (95\% CI 1.4 to 3.8), $p<0.001$ ), as well as the familial risk surveillance population (OR 3.4 (95\% CI 1.1 to 17), $p<0.05$ ). The incidence of ASP did not differ across the surveillance indication populations.

Figure 4. Lesion types detected during the surveillance colonoscopy stratified by surveillance indication (i.e. post-CRC, post-polypectomy and familial risk)

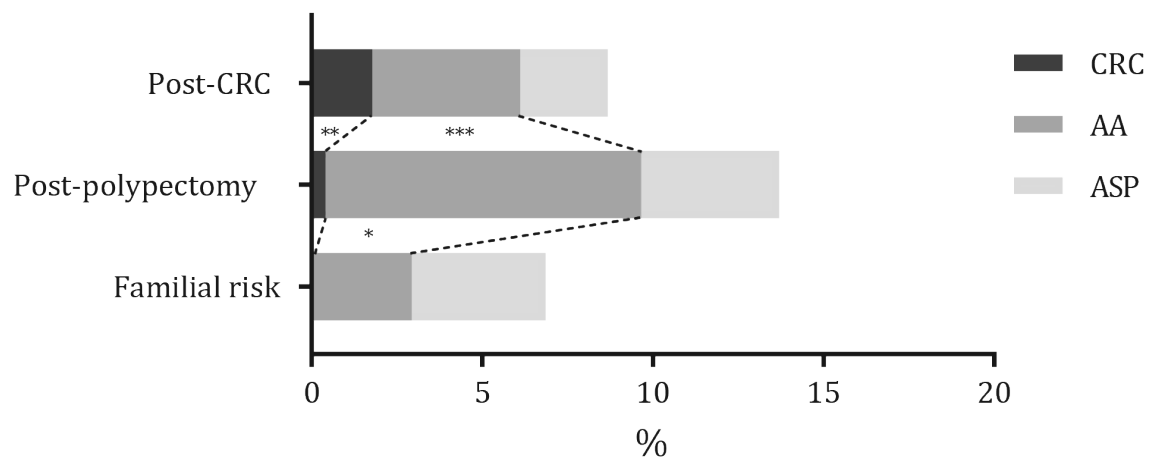

$\mathrm{CRC}=$ colorectal cancer, $\mathrm{A \Lambda}=$ advanced adenoma, $\Lambda \mathrm{SP}=$ advanced serrated polyp Fisher's Exact test, ${ }^{*} \mathrm{p}<0.05,{ }^{* *} \mathrm{p}<0.01,{ }^{* * *} \mathrm{p}<0.001$ 


\section{Discussion}

In this study, we assessed the performance of the multitarget stool DNA test Cologuard in the surveillance setting. When applying relatively high thresholds for test positivity, as currently used for screening, sensitivity of Cologuard for the detection of AN was 59\% and exceeded that of the two FITs (OC Sensor 27\% and FOB Gold 34\%). In particular, Cologuard was more sensitive for detection of advanced precursor lesions. This can be attributed to the DNA marker component of the test, as reflected by the relative low $\mathrm{Hb}$ concentration in the stool sample of patients with AA, and especially with ASP as most advanced lesion. ASP was mostly picked up through the hypermethylation markers of the Cologuard. The FITs, on the other hand, were more specific for non-advanced or negative findings (OC Sensor 89\% and FOB Gold 86\% versus Cologuard 76\%).

The accuracy of the Cologuard was evaluated previously in a large cross-sectional study with average risk individuals. ${ }^{16}$ Even though the same cut-off was used, lower sensitivity for advanced precursor lesions of $42 \%$ was reported (compared to $58 \%$ in the current study), against higher specificity of $87 \%$. Possibly, biological differences in the detected lesions in the surveillance population play a role, such as the relative high contribution of ASP. In contrast, the sensitivity for CRC was higher in the previous study (92.3\%) compared to the current study (77\%). Even though the difference was not significant, a plausible explanation is the early stage of the majority of cancers detected in this surveillance cohort. Nine out of 13 CRC concerned stage I cancers (Supplementary Table 1), which may have less shedding of neoplastic cells compared to more advanced cancers. Also the lower FIT sensitivity for CRC compared to screening studies may be explained by the early stage of the CRC. ${ }^{23}$ However, even though FIT sensitivity is known to improve with cancer stage ${ }^{16,24}$, this has not been described for Cologuard. ${ }^{16}$ Other explanations are different morphological (smaller size or non-polypoid shape) ${ }^{25,26}$ and molecular features ${ }^{27}$ of the tumours encountered during surveillance compared to screeningdetected tumours. The surveillance population consists of patients with increased risk for CRC. These patients are monitored by colonoscopy. 
Thus, when evaluating alternative stool-based surveillance strategies, especially false negative test results are undesirable, while higher rates of false positivity can be accepted. Lowering the screening cut-offs to reach $50 \%$ positivity rate, increased the sensitivity of Cologuard and FITs considerably, thereby reducing the risk of missing AN. In this scenario, a single round of Cologuard would miss $24 \%$ of AN, while FIT would miss a significantly higher proportion of $40 \%$. In practice, the possibility to lower the threshold is constrained by the limit of quantification of the tests. ${ }^{28}$

Alternatively, also repeated testing can increase the likelihood of detecting AN within a given timeframe. Even though delayed detection may be acceptable for AA and ASP considering the slow rate of malignant transformation, for CRC this is debatable. There, diagnostic delay may result in more progressed disease and reduced survival. Because Cologuard is more expensive than FIT $(\sim € 600$ versus $\sim € 20$, respectively), ${ }^{4,29}$ efficiency of repeated testing also needs to be evaluated against test costs. Upon full study completion, cost-effectiveness modelling using the ASCCA (Adenoma and Serrated pathway to Colorectal CAncer) model will be performed to identify the optimal stool-based surveillance strategy. Different intervals between the stool tests will be evaluated. Other factors to consider are logistic issues and ease to perform the test, which influence the number of analytical test drop-outs and participation rates. As can be seen from Figure 1, the number of drop-outs due to analytical problems is almost tenfold higher with Cologuard testing compared to FIT.

In the current study, patients with different surveillance indications, and therefore different risk profiles, were included. According to our subgroup analysis, the a priori risk for CRC is highest in the post-CRC population. Therefore it may be warranted for this subgroup to maintain colonoscopy-based surveillance, especially during the first years after curative resection when CRC risk is most pronounced. ${ }^{30-32}$ Indeed, also in the present study most surveillance-detected CRC $(6 / 9 ; 67 \%)$ occurred within two years after initial CRC diagnosis (Supplementary Table 1). The lower prevalence of AA in the post-CRC population may be explained by the reduced length of the colon still in situ after CRC resection. The post-polypectomy and familial risk subgroups, accounting for $67 \%$ of the population, harboured mostly 
advanced precursor lesions or non-advanced findings.

Considering the relatively high sensitivity of Cologuard for advanced precursor lesions and the possibility of repeated testing, surveillance based on stool testing may prove a suitable alternative for the colonoscopy-based strategy in the final analysis. This study has several limitations.

Firstly, we present an interim analysis including 1,551 of the 4,000 scheduled patients, and therefore the study is still underpowered in determining the accuracy of the stool tests. Secondly, the surveillance population in this study was a mixed group, comprising post-CRC, post-polypectomy and familial risk patients, potentially requiring different surveillance approaches. The study was not powered to perform subgroups analysis on test accuracy. Lastly, we did not evaluate the performance of stool tests over multiple rounds.

Nevertheless, this study is the first to our knowledge to assess multitarget stool DNA testing in the surveillance population and compare it to FIT. Considering the low evidence for the colonoscopy-based strategy and the high colonoscopy demand it generates, alternative strategies are highly warranted. In addition, endoscopists and pathologists were blinded for the test results, as were the laboratory workers for the clinical findings. Because Cologuard and FITs were performed on the same stool sample, direct comparison between the tests was possible. Furthermore, the inclusion of the entire range of surveillance patients can provide insights in test performance in different subgroups.

In conclusion, the multitarget stool DNA test (Cologuard) has higher sensitivity than FIT for detection of advanced neoplasia in the surveillance population. In the surveillance setting, lower specificity can be accepted to increase test sensitivity, while still reducing the number of unnecessary colonoscopies. Mathematical modelling approaches will be applied to the final study data in order to determine an optimal stool-based strategy for surveillance. 


\section{Acknowledgements}

We thank Health RI (Utrecht, the Netherlands) for providing tools for the integration of clinical and test data and for facilitating long-term data storage. We thank the technicians for their invaluable work in assisting with the processing of the stool samples, the research nurses for their efforts to include patients and Pauline van Mulligen for the project management. We also wish to thank the involved personnel at the participating centers, as wel as all participants.

\section{Funding}

This study is financially supported by Alpe d'Huzes, Dutch Cancer Society (reference number: NKI 2013-6338). Exact Sciences (Madison, WI, USA) provided test equipment and performed Cologuard analysis. Sentinel (Milan, Italy) provided equipment and reagents for the FOB Gold analysis. 


\section{References}

1. Bouvier, AM. et al. The lifelong risk of metachronous colorectal cancer justifies long-term colonoscopic follow-up. Eur. J. Cancer 44, 522-7 (2008).

2. Martínez, ME. et al. A pooled analysis of advanced colorectal neoplasia diagnoses after colonoscopic polypectomy. Gastroenterology 136, 832-41 (2009)

3. Atkin, WS., Morson, B. C. \& Cuzick, J. Long-term risk of colorectal cancer after excision of rectosigmoid adenomas. N. Engl. J. Med. 326, 658-62 (1992).

4. Greuter, MJE, de Klerk, C. M., Meijer, G. A., Dekker, E. \& Coupé, V. M. H. Screening for Colorectal Cancer With Fecal Immunochemical Testing With and Without Postpolypectomy Surveillance Colonoscopy. Ann. Intern. Med. 167, 544 (2017)

5. Winawer, SJ. et al. Randomized comparison of surveillance intervals after colonoscopic removal of newly diagnosed adenomatous polyps. The National Polyp Study Workgroup. The New England journal of medicine 328, (1993).

6. Bertario, L. et al. Predictors of metachronous colorectal neoplasms in sporadic adenoma patients. Int. J. Cancer 105, 82-87 (2003)
7. Morris, EJA, et al., Post-colonoscopy colorectal cancer (PCCRC) rates vary considerably depending on the method used to calculate them: a retrospective observational population-based study of PCCRC in the English National Health Service. Gut 64, 1248-1256 (2015)

8. Cottet, V. et al. Long-term risk of colorectal cancer after adenoma removal: a population-based cohort study. Gut 61, 1180-6 (2012).

9. Atkin, W. et al. Adenoma surveillance and colorectal cancer incidence: a retrospective, multicentre, cohort study. Lancet. Oncol. 18, 823-834 (2017).

10. Rabeneck, L. et al. Bleeding and Perforation After Outpatient Colonoscopy and Their Risk Factors in Usual Clinical Practice. Gastroenterology 135, 1899-1906. e1 (2008).

11. Gavin, DR. et al. The national colonoscopy audit: a nationwide assessment of the quality and safety of colonoscopy in the UK. Gut 62, 242-249 (2013).

12. Lieberman, D. Colon-Polyp Surveillance - Do Patients Benefit? N. Engl. J. Med. 371, 860-861 (2014). 
13. Waye, JD. \& Braunfeld, S. Surveillance Intervals after Colonoscopic Polypectomy. Endoscopy 14, 79-81 (1982).

14. Shaukat A, Mongin SJ, Geisser MS, Lederle FA, Bond JH, Mandel JS, C. T. Long-term mortality after screening for colorectal cancer. N Engl J Med. 19, 1106-14 (2013).

15. Atkin, W. et al. Faecal immunochemical tests versus colonoscopy for post-polypectomy surveillance: an accuracy, acceptability and economic study. Health Technol. Assess. (Rockv). 23, 1-84 (2019).

16. Imperiale, TF. et al. Multitarget stool DNA testing for colorectal-cancer screening. N. Engl. J. Med. 370, 1287-97 (2014).

17. van Lanschot, MCJ. et al. Molecular stool testing as an alternative for surveillance colonoscopy: a crosssectional cohort study. BMC Cancer 17, 116 (2017).

18. Nederlandse Richtlijn Coloscopie Surveillance. Available at: http:// www.mdl.nl/uploads/240/1308/ Richtlijn_Coloscopie_Surveillance_ definitief_2013.pdf. (Accessed: 10th February 2015)
19. Lai, E. J., Calderwood, A. H., Doros, G., Fix, O. K. \& Jacobson, B. C. The Boston bowel preparation scale: a valid and reliable instrument for colonoscopy-oriented research. Gastrointest. Endosc. 69, 620-5 (2009).

20. Wieten, E. et al. Equivalent Accuracy of 2 Quantitative Fecal Immunochemical Tests in Detecting Advanced Neoplasia in an Organized Colorectal Cancer Screening Program. Gastroenterology 155, 1392-1399.e5 (2018).

21. Fraser, C. G., Allison, J. E., Halloran, S. P., Young, G. P. \& Expert Working Group on Fecal Immunochemical Tests for Hemoglobin, Colorectal Cancer Screening Committee, World Endoscopy Organization. A Proposal to Standardize Reporting Units for Fecal Immunochemical Tests for Hemoglobin. JNCI J. Natl. Cancer Inst. 104, 810-814 (2012).

22. Sint Nicolaas, J. et al. Optimal resource allocation in colonoscopy: timing of follow-up colonoscopies in relation to adenoma detection rates. Endoscopy 45, 545-52 (2013). 
23. de Wijkerslooth, T. R. et al. Immunochemical Fecal Occult Blood Testing Is Equally Sensitive for Proximal and Distal Advanced Neoplasia. Am. J. Gastroenterol. 107, 1570-1578 (2012).

24. Chiu, H. et al. Association Between Early Stage Colon Neoplasms and False-negative Results From the Fecal Immunochemical Test. Clin. Gastroenterol. Hepatol. 11, 832-838. e2 (2013).

25. le Clercq, C. et al. Interval Colorectal Cancers Frequently Have Subtle Macroscopic Appearance: A 10 YearExperience in an Academic Center. Gastroenterology 140, (2011).

26. Pabby, A. et al. Analysis of colorectal cancer occurrence during surveillance colonoscopy in the dietary Polyp Prevention Trial. Gastrointest. Endosc. 61, 385-91 (2005).

27. Stoffel, E. M. et al. Clinical and Molecular Characteristics of Post-Colonoscopy Colorectal Cancer: A Population-based Study. Gastroenterology 151, 870-878.e3 (2016).

28. Halloran, S., Launoy, G. \& Zappa, M. European guidelines for quality assurance in colorectal cancer screening and diagnosis. - First Edition Faecal occult blood testing. Endoscopy 44, SE65-SE87 (2012).
29. How much does Cologuard cost? Via: https://www.cologuardtest.com/ faq/cost. (23rd July 2018)

30. Mulder, S. A. et al. The incidence and risk factors of metachronous colorectal cancer: an indication for follow-up. Dis. Colon Rectum 55, 522-31 (2012).

31. Kahi, C. J. et al. Colonoscopy surveillance after colorectal cancer resection: recommendations of the US multi-society task force on colorectal cancer. (2016).

32. van Lanschot, M. C. J. et al. Yield of Surveillance Colonoscopies 1 Year After Curative Surgical Colorectal Cancer Resections. Clin. Gastroenterol. Hepatol. (2019). 


\section{Supplementary material}

Supplementary Figure 1. ROC curve comparing Cologuard with FIT for advanced neoplasia and colorectal cancer detection, using different control groups

A. Advanced neoplasia controls: patients with non-advanced or lesser finding

Test - a. Cologuard $-b$. oc Sensor $-c$. FoB Gold

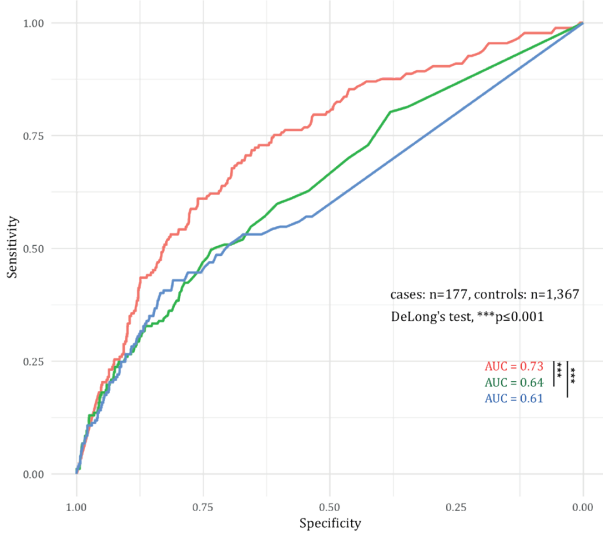

C. Colorectal cancer controls: patients with non-advanced or lesser findings Test - a.Cologuard - b. oc Sensor $-c$. FOB Gold

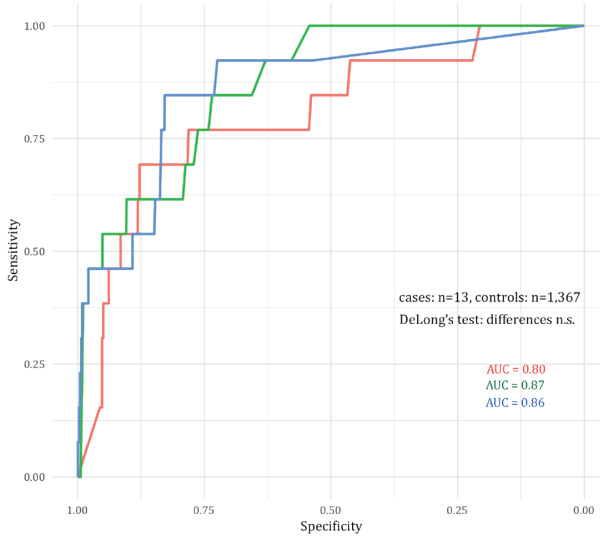

B. Advanced neoplasia controls: patients without any lesions Test - a. Cologuard - b. oc Sensor - c. FoB Gold

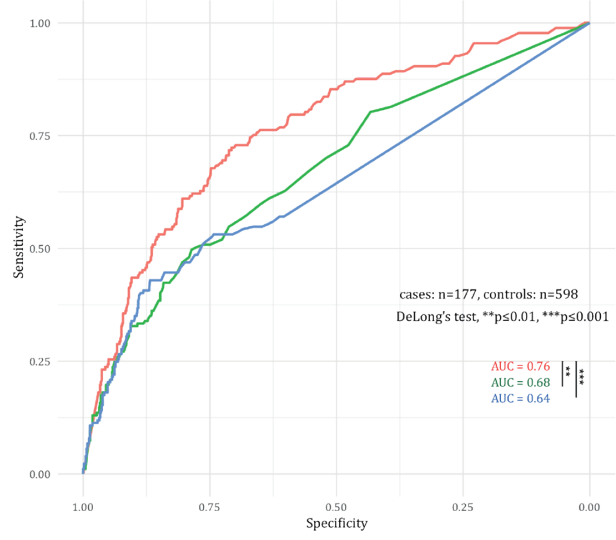
D. Colorectal cancer controls: patients without any lesions
Test - a.Cologuard - b. OCSensor - c. FOB Gold

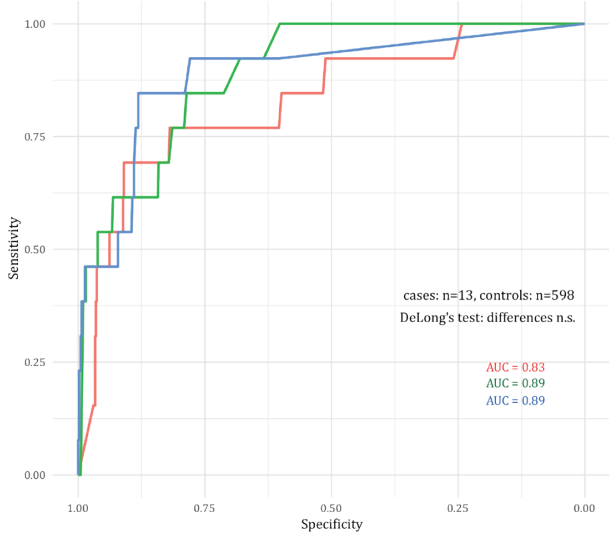


Supplementary Table 1. Details of patients with colorectal cancer at the current surveillance colonoscopy

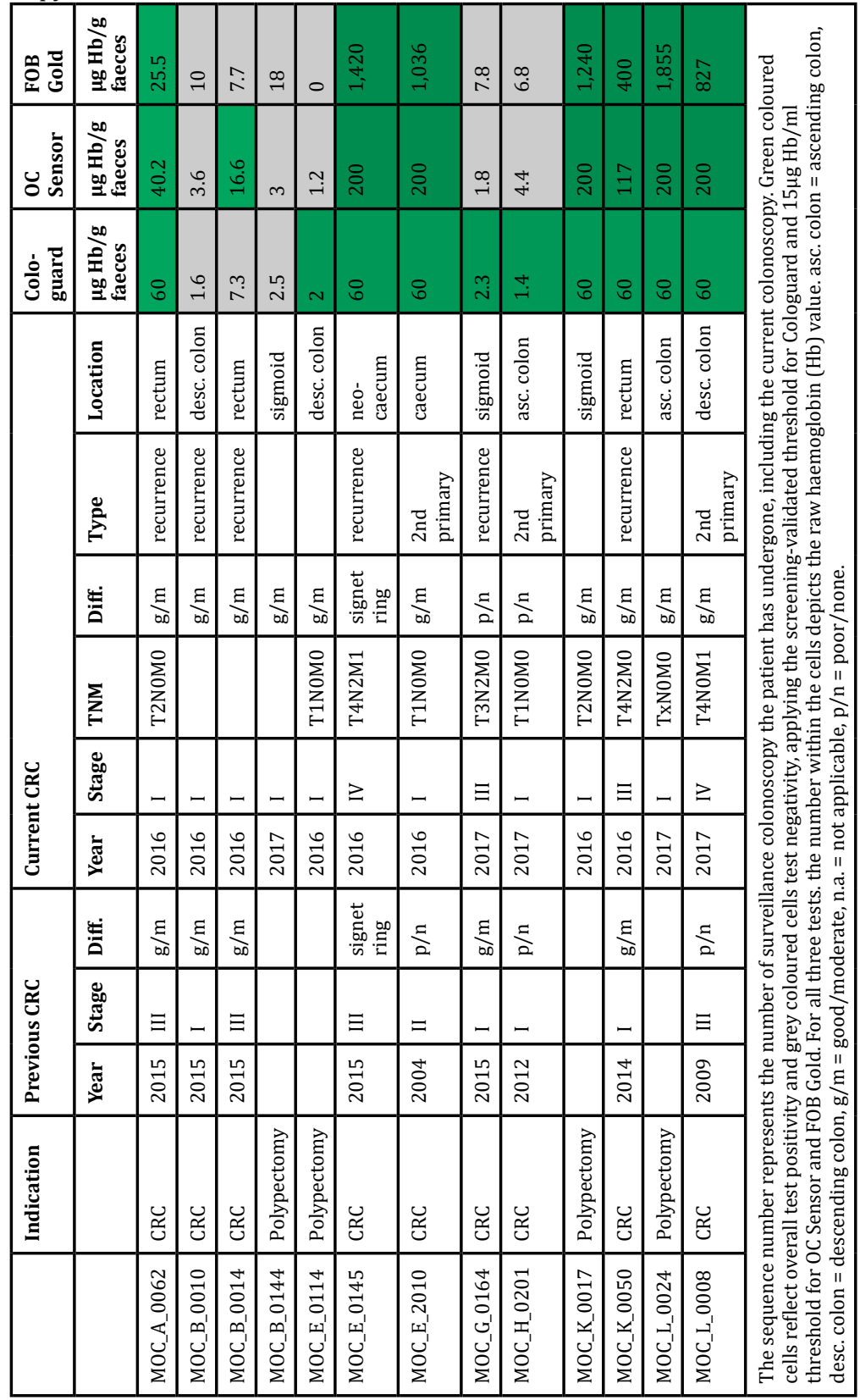





\section{CHAPTER 7}

\section{Impact of endoscopist training on the postcolonoscopy colorectal cancer rate}

Alouisa J.P. van de Wetering, Roel M.M. Bogie, Chantal M.C. Le Clercq, Ad A.M. Masclee, Silvia Sanduleanu

Part of this is published in Gastrointestinal Endoscopy Gastrointestinal endoscopy 2017;85(5):1113-4 


\section{Summary}

\section{Introduction}

Post-colonoscopy colorectal cancers (PCCRCs) are considered to be a crucial quality outcome measure for colonoscopy. PCCRCs may occur due to various reasons such as non-adherence to surveillance intervals, inadequate bowel examination, incomplete polyp resection, missed lesions or newly developed cancers. Recent studies indicate that non-polypoid colorectal neoplasms (NP-CRNs) and serrated lesions, which are smaller and more easily overlooked during colonoscopy, may be important precursor lesions of PCCRC. The present study was undertaken to investigate whether systematic training in detection and resection of NP-CRN, reduces the PCCRC rate.

\section{Methods}

In the Maastricht UMC+, all endoscopists were systematically trained in 2008 to optimize detection and resection of NP-CRNs. PCCRCs found after colonoscopy performed 4 years before and 4 years after training were included. For PCCRC identification, we used follow-up data until 2014.

\section{Results}

After training PCCRC rate decreased by more than 50\% from 2.0 per 1000 colonoscopies to 0.8 per 1000 colonoscopies. Of the 11 PCCRCs found after training, 45\% was associated with missed lesions, $18 \%$ due to non-adherence to surveillance intervals, $18 \%$ due to newly developed CRC, $18 \%$ due to inadequate bowel examination and none due to incomplete polyp resection.

\section{Conclusion}

Systematic training in detection and resection of NP-CRNs appears to be able to reduce the PCCRC rate. However, to further minimize the PCCRC rate a multifactorial approach is needed, where training, but also adherence to surveillance intervals and employing quality indicators for colonoscopy are of importance. 


\section{Introduction}

It has been clearly shown that population based colorectal cancer screening either by fecal testing and/or colonoscopy significantly reduces CRC incidence and mortality. ${ }^{1,2}$ Although colonoscopy is considered as gold standard method for polyp detection and removal, it should be noted that colonoscopy is not perfect since still some CRCs are detected after a negative colonoscopy. ${ }^{3}$ For a CRC screening program to be effective, the occurrence of these so-called post-colonoscopy colorectal cancers (PCCRCs) should be as low as possible. Several factors influence sensitivity of colonoscopy and thus occurrence of PCCRCs; for instance image quality of the colonoscopes (high definition), bowel cleansing and skills of the endoscopist. ${ }^{4}$ This way, the occurrence of PCCRCs is an important quality outcome parameter for CRC screening programs.

It is known that PCCRCs occur due to various reasons (non-adherence to surveillance intervals, inadequate bowel examination, incomplete polyp resection, missed lesions or newly developed cancers $)^{5}$, where previous studies showed that a large subset of PCCRCs is due to missed lesions ${ }^{6,7}$ and could thereby be preventable.

It could be hypothesized that precursor lesions of PCCRCs are often non-polypoid colorectal neoplasms (NP-CRNs) or serrated lesions, which are known to be mainly located in the proximal colon and have a flat appearance. ${ }^{8,9}$

This hypothesis is based on previous studies showing PCCRCs harbor these same characteristics; often located in the proximal colon, a flat appearance and smaller in $\operatorname{size}^{6,10}$, making them more difficult to detect and resect. ${ }^{3,8}$ In addition, a subset of NPCRNs and serrated lesions have different molecular features (compared to polypoid neoplasms), which may be more closely associated with carcinoma. ${ }^{11,12}$

Taken together, PCCRCs are likely to derive from non-polypoid/serrated lesions, which are more challenging to detect and resect. In order to prevent PCCRCs, training and advanced endoscopic skills are required. 
McGill et al. investigated the effect of endoscopy training in a longitudinal assessment (ranging from 200 to 1600 performed colonoscopies). Over time, an increase in the detection of NP-CRNs was seen, indicating that non-polypoid adenoma detection is a skill that can be learned, but does require time and effort. ${ }^{13}$ Up to now, no study has explored whether systematic training in detection and resection of NP-CRNs directly leads to reduction in PCCRC rate. Aim of the present study was to examine PCCRC rate and PCCRC etiology before and after implementation of a short systematic training program for the detection and resection of NP-CRN.

\section{Methods}

\section{Data collection}

At the Maastricht University Medical Center, a specific training program for detection and resection of non-polypoid neoplasms was initiated in 2008. A specific aim of that training program was to increase awareness and detection of NP-CRNs. All endoscopists, faculty, and trainees at our university hospital were trained in a systematic training program comprising of lectures to improve awareness and basic knowledge, learning from experts, videos, cases and individual feedback. ${ }^{14}$

From there, a prospective cohort study was initiated, including all colonoscopies performed between February 2008 and February 2012. Patients with hereditary CRC syndromes or history of CRC were excluded, as well as patients with inflammatory bowel disease or patients younger than 18 years of age.

All new diagnosed CRC cases from January 2004 to October 2014 were retrieved using the Nationwide Pathology Database (PALGA). We cross-linked detailed patient information from the hospital registries to endoscopic and pathological reports (Figure 1). In that way we were able to identify post-colonoscopy colorectal cancers (PCCRCs). In addition, all CRC cases found in the prospective database were also screened for PCCRCs. Year of the last negative colonoscopy was used to compare PCCRC incidence pre- and post-training. 
Figure 1. Overview of study and the data sources used.

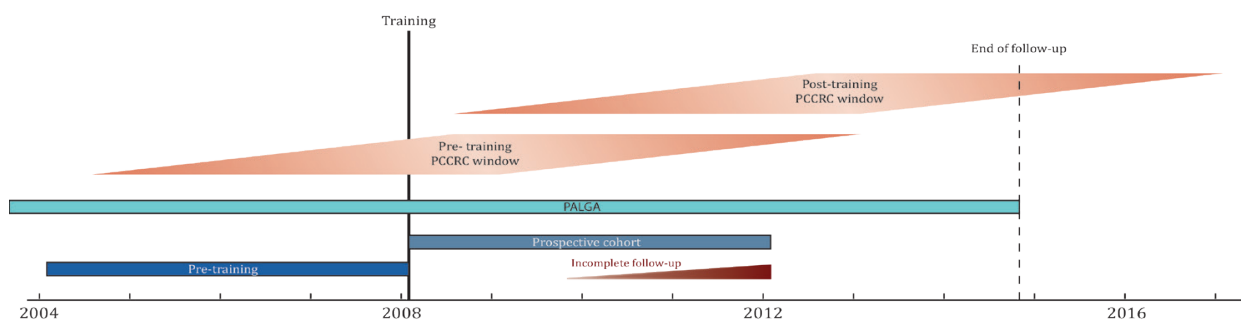

\section{Definition and statistical analysis}

PCCRCs were defined as a CRC diagnosed 6 to 60 months after a colonoscopy that was negative for CRC. PCCRC rate after training was determined by the number of PCCRCs divided by the total number of colonoscopies (including surveillance colonoscopies), as described by Morris et al. ${ }^{15}$ Patient years of follow-up (PYFU) were calculated using the time between index colonoscopy and end of follow-up (October 2014).

Prior to training, only data on the number of colonoscopies per year were available. To estimate the PYFU we assumed that prior to training, the subset of index colonoscopies and mean follow-up was the same as in 2008 (i.e. both pre- and posttraining 48.5 months). As sensitivity analysis we assumed complete follow-up (i.e. 60 months) for colonoscopies prior to training instead of the estimated 48.5 months. A Z-test was performed to compare PCCRC rates before and after training. A p-value $<0.05$ was considered statistically significant.

The other outcome parameter was the etiology of the PCCRCs, together with clinical and pathological characteristics (i.e. size, location, macroscopic appearance and histopathology). Location was subdivided in proximal colon (cecum to splenic flexure) and distal colon (descending colon to rectum). 
The PCCRCs were classified using the Pabby algorithm ${ }^{5}$ modified by Huang et al. $^{16}$ (Figure 2). Based on findings at the index-examination, time between colonoscopy and CRC diagnosis, location and stage of the tumor at diagnosis, the PCCRCs were categorized on most likely etiology: non-adherence to surveillance intervals, inadequate examination/surveillance, incomplete resection, missed lesions or newly developed CRC.

This study was approved by the Institutional Review Boards of the Maastricht University Medical Center and is registered in the Netherlands Trial Registry: NTR4844. Approval for the prospective colonoscopy database is assigned by the METC of the Maastricht University Medical Center (MUMC) with number 14-4-046. The need for informed consent was waived by the Institutional Review Board.

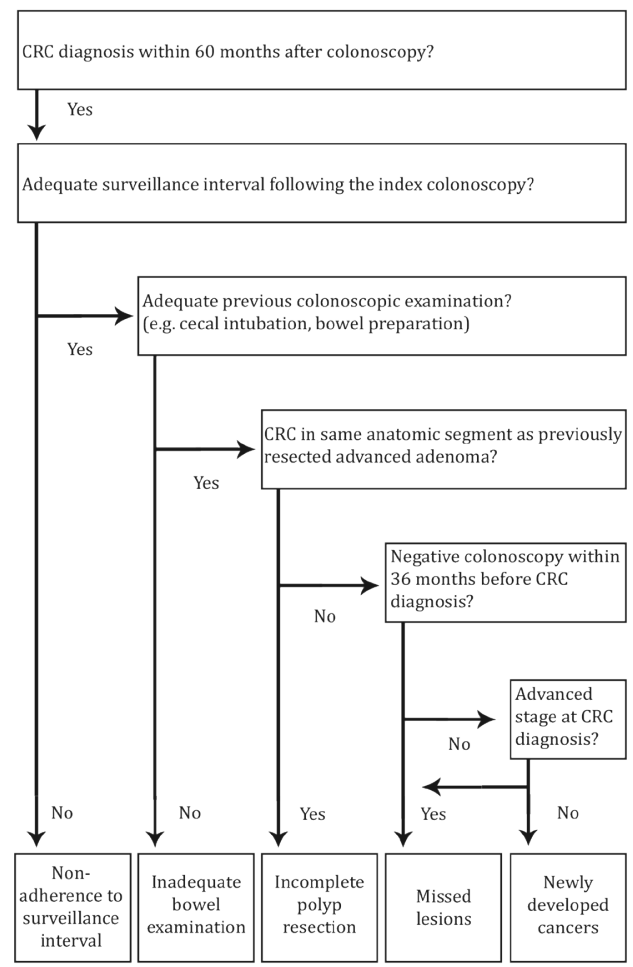

Figure 2. Modified algorithm by Pabby et al. $^{5}$ evaluating the most likely etiology of postcolonoscopy colorectal cancers (PCCRC) ${ }^{16}$ 


\section{Results}

The post training cohort of 9,353 patients was enrolled from a total of 13,190 colonoscopies in four years (2008-2012). Patients underwent elective colonoscopy for symptoms, surveillance or screening. Patients with IBD, hereditary CRC or a history of CRC were excluded $(n=1,136)$. The analyzed post-training population consisted of 8,217 patients with a mean age of 59.1 years (SD 15.8) and of which $54 \%$ is female.

Prior to training (2004-2008), 8,468 colonoscopies were performed. Based on the findings of 2008, we estimated that 5,275 unique patients would have had one or more colonoscopies during this period of four years. We assumed that all patients of this study period had 48 months of follow-up, since this was the case for the patients included in 2008. With this information, we calculated the total years of follow-up (PYFU) for the pre-training cohort (2004-2008) which was 21,299 person years. The total years of follow-up for patients from the post-training prospective cohort (2008-2012) was measured at 31,993 person years.

When comparing the PCCRC rates before and after training, this study shows that the PCCRC rate declined after implementation of training by more than $50 \%$ from 2.0/1000 colonoscopies before training to $0.8 / 1000$ colonoscopies after training $(p=0.031)$. These rates result in an estimated decrease from $0.79 / 1000$ PYFU before training to $0.34 / 1000$ PYFU after training ( $p=0.041)$ (Figure 3).

A total of 11 patients (mean age (SD) 76.8 years (7.4); 45\% female) were diagnosed with PCCRCs after colonoscopy between February 2008 and February 2012, i.e. in the post-training cohort.

Clinical characteristics of these PCCRC cases are shown in Figure 4. From the PCCRC patients, ten patients were older than 70 years. The time to CRC diagnosis varied from 14 to 52 months. In contrast with previous data, PCCRCs were more equally distributed over the proximal and distal colon. 
Nine patients had diverticular disease and two patients had proven metastatic disease at time of diagnosis. Indication for index colonoscopy were symptoms (54.5\%) (i.e. rectal blood loss, anemia) or surveillance (45.5\%).

Using the Pabby algorithm (Figure 2), potentially missed lesions remained the main explanation (45.4\%) for the occurrence of PCCRC, while no PCCRC case could be attributed to incomplete resection. Non-adherence to surveillance intervals comprised $18.2 \%$ of cases, as well as inadequate bowel examination (18.2\%) and newly developed cancers (18.2\%).

Applying the 60 month follow-up as a sensitivity analysis, the PCCRC rate before training is estimated at $0.64 / 1000$ PYFU. When comparing to the PCCRC rate after training $(0.34 / 1000$ PYFU) the difference is less distinctive $(p=0.144)$.

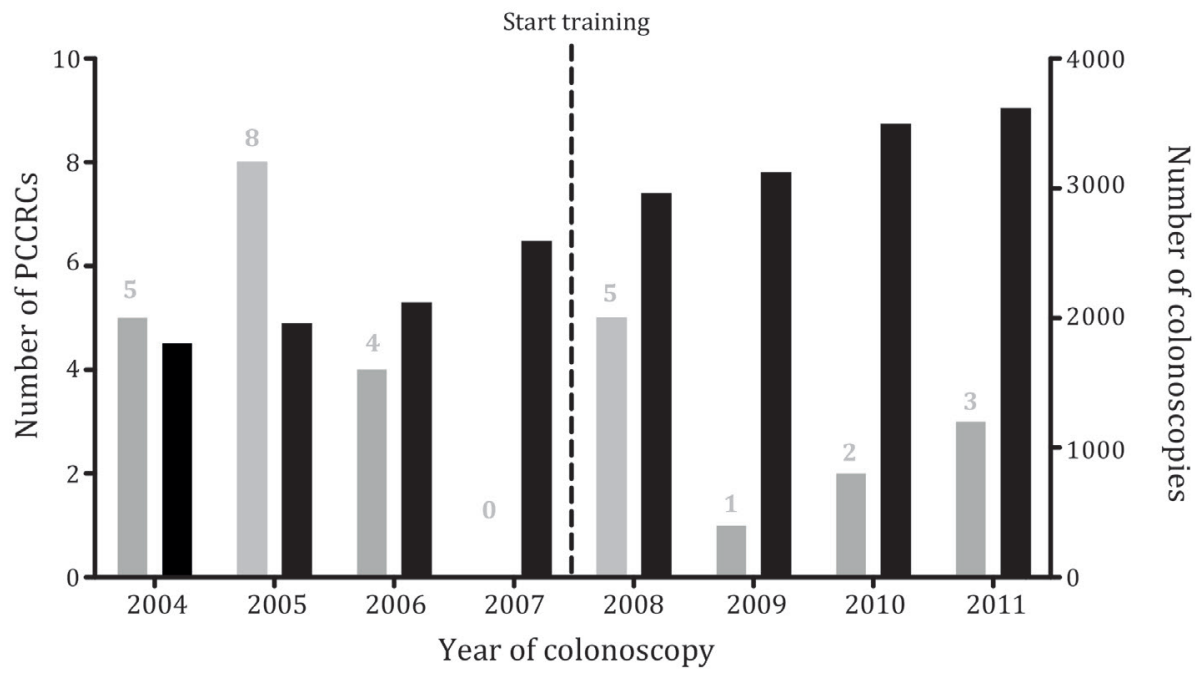

Total number of colonoscopies

PCCRCs: number of colonoscopies followed by CRC diagnosis after 6-60 months

Note: Cumulative PCCRC rate decreased from 2.0 per 1000 colonoscopies before training to 0.8 per 1000 colonoscopies after training

Figure 3. Number of yearly postcolonoscopy colorectal cancers (PCCRC) and number of colonoscopies before and after systematic endoscopists training (February 2004-February 2012) 
PCCRC RATE AFTER TRAINING

Figure 4. Clinical characteristics of the patients with a PCCRC from 2008-2012

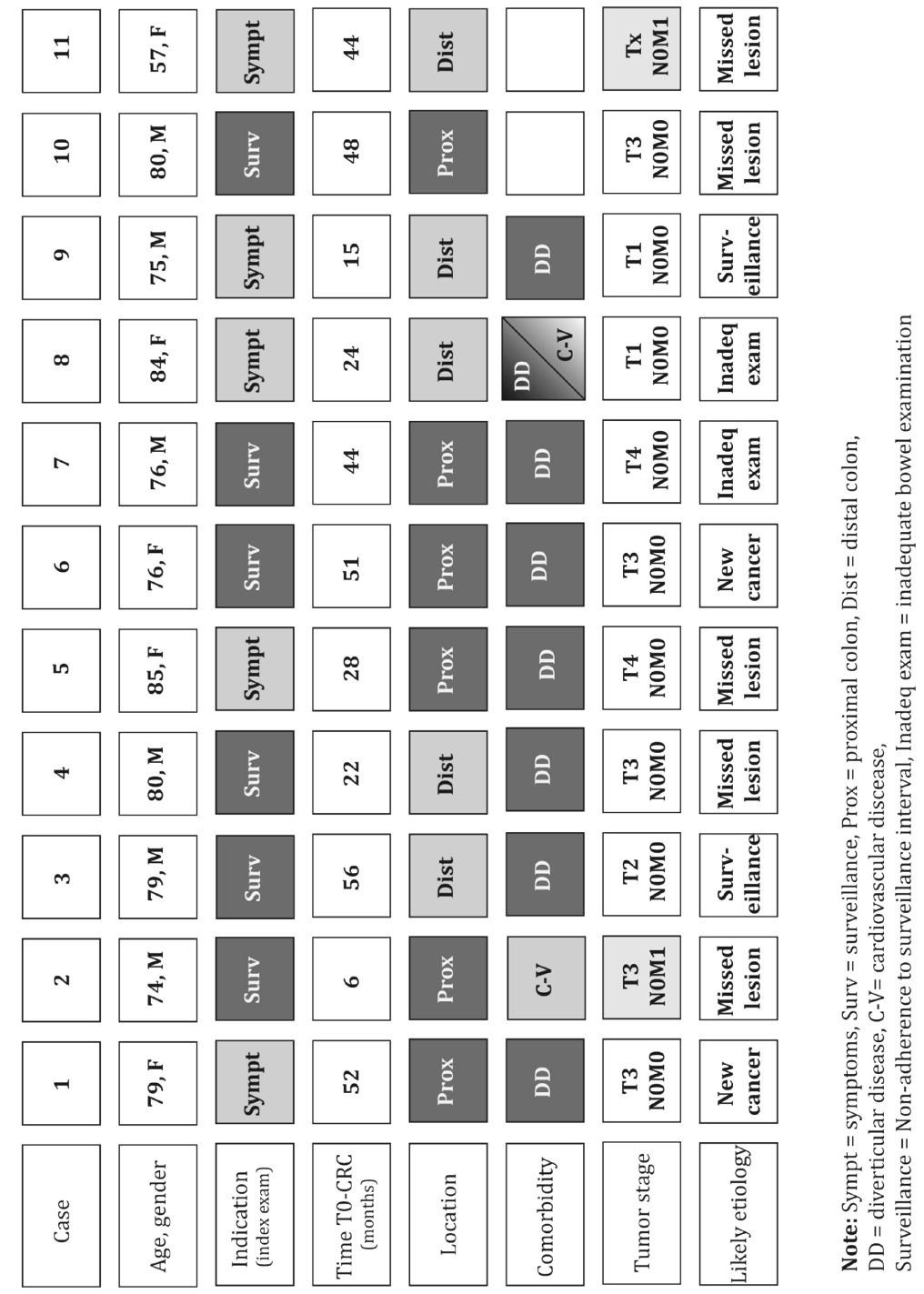




\section{Discussion}

We observed a more than 50\% decrease in PCCRCrate from 2.0 per 1000 colonoscopies before training to 0.8 per 1000 colonoscopies after systematic endoscopist training. The majority of PCCRCs (82\%) appeared to be potentially preventable, as they were attributable to missed lesions, nonadherence to surveillance intervals and inadequate bowel examination.

Nayor et al. have previously shown that non-adherence to surveillance intervals may increase the number of PCCRC cases, especially in cases where bowel preparation at index examination was inadequate. ${ }^{17}$ Apart from non-adherence to surveillance intervals and inadequate bowel preparation, several studies have shown that over $50 \%$ of PCCRCs can be attributed to missed lesions and up to $20 \%$ to incomplete resection..$^{6,7}$

In line with our results, other studies on education and training in quality of polyp detection and endoscopic resection have shown that colonoscopy quality parameters generally improve after training. ${ }^{18,19}$ Coe et al. ${ }^{18}$ have shown that for adenoma detection rate (ADR) and Kaminski et al. ${ }^{19}$ for ADR and also for detection of non-polypoid lesions. On the other hand, Shaukat et al. ${ }^{20}$ did not observe a significant change in adenoma detection rate of individual endoscopists despite implementation of a training program.

Based on previous research, PCCRCs are more likely to be proximally located, smaller in size and have a flat macroscopic appearance compared to prevalent CRCs. Together with the knowledge that adequate bowel preparation is more difficult to achieve in the proximal colonic area, these findings suggest that PCCRCs could have originated from precursors which have been overlooked more easily during index colonoscopy. 6,7

In the current study, the 11 PCCRC cases we analyzed were equally distributed over the colon. This could be the result of fewer missed proximally located lesions after training. 
In line with previous data, patients with PCCRC are usually older compared to prevalent CRC cases and have substantially more comorbidity, in particular diverticular disease ${ }^{21,22}$, which makes the performance of a complete colonoscopy far more difficult.

Missed lesions were the most common cause of PCCRC (45\%) in our study, a finding that is in line with previous data. ${ }^{3,7,23}$ In another Dutch population based study on PCCRCs (total $n=147$ ) performed by our group, $13.6 \%$ of the PCCRCs were attributable to newly developed cancers ${ }^{6}$, comparable with the results in the present study (18.2\%). Another preventable factor is inadequate bowel examination (e.g. no cecal intubation or insufficient bowel preparation). Nowadays quality indicators for colonoscopy have been introduced and implemented to assure the quality of each colonoscopic intervention. ${ }^{4}$

Incomplete resected polyps have been reported as contributor to PCCRCs in up to $19 \%$ of cases. ${ }^{7,24,25}$ In the present study, no PCCRCs could be attributed to incomplete resection. Non-adherence to surveillance interval accounted for $18 \%$ of our PCCRCs, a percentage that is higher compared to other studies. ${ }^{26}$ It should be noted that adherence to surveillance intervals is a shared responsibility of patients and of physicians and endoscopy staff. ${ }^{17}$

Some strengths and limitations of our study should be mentioned. First, this study is unique because of availability of colonoscopy data and CRC data prior to and after systematic training of endoscopists. Second, a detailed description of all PCCRCs is available. In a study on PCCRC, CRC and number of colonoscopies covering the whole South-Limburg region (including three large hospitals), we found that PCCRC rate in the decade prior to systematic endoscopist training accounted for approximately 2.0 per 1000 colonoscopies $^{6}$, identical to the rate found prior to training in the present study. Since this PCCRC rate showed no significant decline over time, this observed percentage validates the pre-training cohort estimations and underlines the difference between pre-and post-training PCCRC percentages. 
It is however important to acknowledge that bias may have occurred. First, the follow-up ended in October 2014. Therefore, regarding the colonoscopies performed after October 2009, the occurrence of late onset PCCRCs cannot be excluded with certainty. In this way especially 'newly developed' PCCRCs will be missed, which is less influenced by training. To limit this bias, the rate of PCCRCs pre- and posttraining were presented as PCCRCs/1000 person years of follow-up.

Second, person years of follow-up prior to training were based on estimation, resulting in bias in case the real follow-up would be longer. To overcome this bias, a sensitivity analysis was performed using 60 months of follow-up in the cohort prior to training.

Finally, data were prospectively collected post-training resulting in validation of the PALGA dataset since additional PCCRCs may be identified. In the retrospectively collected pre-training data this additional check was not available, theoretically leading to a small chance of underreporting PCCRCs.

Taken into account these limitations, our data point out that focusing on endoscopist related factors such as systematic short-interval training for the recognition and complete resection of NP-CRN may help to minimize PCCRC rate.

In conclusion, we have shown a decrease in PCCRC rate after implementation of systematic training in NP-CRN detection and resection by more than $50 \%$. The etiology of the PCCRCs lies mostly in missed lesions, and in a lesser extent to nonadherence to surveillance intervals, inadequate bowel examination and newly developed cancers. So, there is room for further improvement.

In order to further minimize the PCCRC rate it should be acknowledged that a multifactorial approach is needed giving attention to training in detection and resection of polyps, especially of NP-CRNs, adherence to colonoscopy surveillance intervals and employing quality indicators for colonoscopy. 


\section{References}

1. Mandel JS, et al. The effect of fecal occult-blood screening on the incidence of colorectal cancer. $\mathrm{N}$ Engl J Med 2000;343(22):1603-7.

2. Shaukat A, et al. Long-term mortality after screening for colorectal cancer. N Engl J Med 2013;369(12):110614.

3. le Clercq CM, Sanduleanu S. Interval colorectal cancers: what and why. Curr Gastroenterol Rep 2014;16(3):375.

4. Rex DK, et al. Quality indicators for colonoscopy. Am J Gastroenterol 2015;110(1):72-90.

5. Pabby A, et al. Analysis of colorectal cancer occurrence during surveillance colonoscopy in the dietary Polyp Prevention Trial. Gastrointest Endosc 2005;61(3):385-91.

6. le Clercq CM, et al. Postcolonoscopy colorectal cancers are preventable: a population-based study. Gut 2014;63(6):957-63.

7. Robertson DJ, et al. Colorectal cancers soon after colonoscopy: a pooled multicohort analysis. Gut 2014;63(6):949-56.

8. Rondagh EJ, et al. Endoscopic appearance of proximal colorectal neoplasms and potential implications for colonoscopy in cancer prevention. Gastrointest Endosc 2012;75(6):1218-25.
9. Hazewinkel Y, et al. Endoscopic features of sessile serrated adenomas: validation by international experts using high-resolution white-light endoscopy and narrow-band imaging. Gastrointest Endosc 2013;77(6):916-24.

10. Sanduleanu $S$, et al. Interval cancers after colonoscopyinsights and recommendations. Nat Rev Gastroenterol Hepatol 2012;9(9):550-4.

11. Voorham QJ, et al. Tracking the molecular features of nonpolypoid colorectal neoplasms: a systematic review and meta-analysis. Am J Gastroenterol 2013;108(7):1042-56.

12. Soetikno RM, et al. Prevalence of nonpolypoid (flat and depressed) colorectal neoplasms in asymptomatic and symptomatic adults. JAMA 2008;299(9):1027-35.

13. McGill SK, et al. The learning curve for detection of non-polypoid (flat and depressed) colorectal neoplasms. Gut 2015;64(1):184-5.

14. Sanduleanu S, et al. Development of expertise in the detection and classification of non-polypoid colorectal neoplasia: Experiencebased data at an academic GI unit. Gastrointest Endosc Clin N Am 2010;20(3):449-60. 
15. Morris EJ, et al. Post-colonoscopy colorectal cancer (PCCRC) rates vary considerably depending on the method used to calculate them: a retrospective observational population-based study of PCCRC in the English National Health Service. Gut 2015;64(8):1248-56.

16. Huang $Y$, et al. Risk and cause of interval colorectal cancer after colonoscopic polypectomy. Digestion 2012;86(2):148-54.

17. Nayor J, et al. Impact of Physician Adherence to Colonoscopy Surveillance Guidelines on Interval Colon Cancer. Gastrointest Endosc 2016.

18. Coe SG, et al. An endoscopic quality improvement program improves detection of colorectal adenomas. Am J Gastroenterol 2013;108(2):219-26; quiz 27.

19. Kaminski MF, et al. Leadership training to improve adenoma detection rate in screening colonoscopy: a randomised trial. Gut 2016;65(4):616-24.

20. Shaukat A, et al. Variation in detection of adenomas and polyps by colonoscopy and change over time with a performance improvement program. Clin Gastroenterol Hepatol 2009;7(12):1335-40.
21. Singh $\mathrm{H}$, et al. Rate and predictors of early/missed colorectal cancers after colonoscopy in Manitoba: a population-based study. Am J Gastroenterol 2010;105(12):258896.

22. Bressler B, et al. Rates of new or missed colorectal cancers after colonoscopy and their risk factors: a population-based analysis. Gastroenterology 2007;132(1):96102.

23. Gorski TF, et al. Colorectal cancer after surveillance colonoscopy: false-negative examination or fast growth? Dis Colon Rectum 1999;42(7):877-80.

24. Kaminski MF, et al. Quality indicators for colonoscopy and the risk of interval cancer. N Engl J Med 2010;362(19):1795-803.

25. Pohl H, et al. Incomplete polyp resection during colonoscopyresults of the complete adenoma resection (CARE) study. Gastroenterology 2013;144(1):7480 e1.

26. Rustagi T, Anderson JC. Endoscopyrelated factors contributing to postcolonoscopy colorectal cancers. Gastroenterology 2014;146(1):3113 ; discussion 3 . 
PCCRC RATE AFTER TRAINING 



\section{CHAPTER 8}

General discussion \&

Concluding remarks 


\section{General discussion}

Colorectal cancer (CRC) is the third leading type of cancer worldwide ${ }^{1}$, with 14.1 million estimated new cases and over 8.2 million deaths in $2012 .{ }^{2}$ In 2030, the global burden of CRC is expected to increase by $60 \%{ }^{3}$, illustrating the impact of this disease and the importance to optimize CRC diagnosis and treatment.

Since it is known that CRC derives from precursor lesions (e.g. adenomas) and the time from adenoma to symptom-detected CRC is 10 to 25 years ${ }^{4}$, early detection and resection of these precursor lesions can reduce incidence, morbidity and mortality of CRC. ${ }^{5}$

In this thesis we have focused on ways to optimize quality and efficacy of CRC diagnosis and endoscopic therapy. Four relevant steps in this process have been identified and explored in more detail: pre-selection of patients, colonoscopy, minimal invasive treatment and surveillance.

\section{Pre-selection}

Colonoscopy is a costly and invasive procedure, which is burdensome for the patient. ${ }^{6}$ It is important to adequately select the patients that should receive a colonoscopy. In order to optimize the pre-selection process and thereby decrease the number and burden of colonoscopy, several non-invasive methods are currently being studied.

In the majority of the CRC screening programs, fecal immunochemical tests (FIT) are used for pre-selection of patients who are invited for colonoscopy. ${ }^{7.9}$ For CRC, FIT shows sensitivities ranging between $68-87 \%$ and specificity of $96-98 \% .^{10,11}$ However, for advanced adenomas, i.e. precursor lesions, the FIT sensitivity is only $23-40 \%{ }^{12,13}$ Therefore, alternative strategies to optimize pre-selection of screenees are worthwhile to investigate. 
On a molecular level, CRC should be considered as a collective term since it is highly heterogeneous. In general, the molecular pathogenesis of CRC is associated with the accumulation of genetic (e.g. mutations of BRAF, KRAS, and $p 53$ and microsatellite instability, copy number alterations) and epigenetic (e.g. DNA methylation of CpG island promotor regions and microRNAs) and biochemical alterations, leading to changes in tumor suppressor genes and oncogenes that transform cells and promote tumor progression. ${ }^{14}$ Since these molecular alterations often dictate natural history, treatment response and outcome, it is suggested that CRC molecular subtyping will help to better identify CRC subtypes. ${ }^{15}$ The four consensus molecular subtypes (CMS) are based on distinguishing biological features and may form the basis for future clinical stratification. ${ }^{15,16}$

In this thesis we focus on epigenetics, and more specifically DNA methylation, which appears to be promising as diagnostic marker. Although $\mathrm{CpG}$ islands represent only $\sim 1 \%$ of the human genome, it covers more than half of all genes. ${ }^{17-19}$ Therefore, the role of DNA methylation markers in CRC diagnosis deserves further investigation.

So far, much has been published on DNA methylation biomarkers to improve or complement FIT, but clinical translation is limited. In chapter 2 current evidence for diagnostic CRC DNA methylation biomarkers is presented. This systematic literature search included 273 articles with 389 markers, of which only 23\% was studied in bodily fluids and only $0.8 \%$ was translated into a clinical test. Among the included studies, the most commonly used bodily fluid for diagnostic DNA methylation markers is blood, which can be considered minimal invasive. Since blood samples cannot easily be obtained at home, from a practical point of view blood-based biomarkers are less suitable for screening purposes. The logistics of non-invasive specimen such as stool or urine are easier as these can be obtained at home, leading to a lower patient burden. 
Besides epigenetic mechanisms involving DNA, microRNA (miRNA) has been associated with carcinogenesis. In case of dysregulation of miRNA expression, cancer may occur. ${ }^{20,21}$ MiRNA can be isolated from bodily fluids (blood and stool), therefore it harbors potential as a diagnostic CRC marker. ${ }^{22}$

Other potential sources for diagnostic CRC markers are currently also being explored. With proteomics (study of the proteome and its modifications), different biomarkers, i.e. diagnostic, prognostic and for treatment response or resistance, are discovered in specimen form several origins (blood, stool, biopsies). In the near future, proteomics might be used more widely for CRC in clinical practice and to promote personalized medicine. ${ }^{23}$

Metabolomics (analysis of metabolic products and processes in bodily fluids and excreta) might also be of value in CRC diagnosis, for example Volatile Organic Compound (VOC), which are present in the gas phase of excreted materials (e.g. breath). A recent review on VOCs and CRC shows that derangement of metabolomics relates to the presence of $\mathrm{CRC}$, where sensitivity ranges between $30-94 \%$ and a specificity of $60-94 \% .^{24}$

The large amount of studies performed on (early) CRC diagnosis and the number of various potential (non-invasive) markers stresses the importance and relevance of this research field. However, in chapter 2 it was observed that in DNA methylation biomarker research, independent validation is lacking and there was large methodological and technical heterogeneity. The same however holds true for the above-mentioned markers.

For example with the promising results of the VOC analyses ${ }^{24}$, various different analytical techniques are being used. Unfortunately, standardized experimental procedures are lacking. 
When starting a study, it would be best if the steps towards clinical translation are described. First, a clinically relevant research question needs to be defined. Next, the study design, specimen, cohort and performance measure need to be defined with the aim of complementing currently available evidence and facilitating external validation or meta-analyses. This will increase the level of evidence. If the results are compared to current gold standard methods, translation towards clinical practice will be enhanced and the research value will be increased.

\section{Surveillance}

In the Netherlands, current surveillance program for prevention of colorectal cancer is colonoscopy-based. This is already accounting for $25 \%$ of the total colonoscopy capacity and is rapidly expanding based on current guidelines for polyp surveillance. ${ }^{25}$ The question has arisen whether the value of colonoscopy-based surveillance should be maintained on top of the nationwide CRC screening. Where FIT-screening reduced CRC mortality by $50.4 \%$, adding colonoscopy-surveillance to FIT screening reduced mortality by an additional $1.7 \%$ to $52.1 \%$, while costs significantly increased. ${ }^{26}$

Since colonoscopy is an invasive and burdensome procedure, diagnostic biomarkers, i.e. DNA methylation markers currently used for screening purposes, may play a role in surveillance strategies.

In the United States, the multitarget stool DNA test (mt-sDNA test) Cologuard® is commercially available. In addition to a hemoglobin immunoassay this test includes the DNA methylation markers NDRG4 and BMP3 and KRAS mutation markers. In asymptomatic, average risk patients, this mt-sDNA test showed significantly higher sensitivities for CRC compared to FIT (92.3\% vs. 73.8\%, $p=0.002$ ) but also had more false positive results (76.4\% vs. $67.2 \%){ }^{27}$

In chapter 6 we investigated whether the mt-sDNA test could be used to pre-select the group of patients with precursor lesions, i.e. advanced neoplasia (AN), for surveillance colonoscopy. ${ }^{28}$ 
To implement diagnostic DNA methylation markers in clinical practice for precursor lesion and early CRC diagnosis, comparison against current methods is needed. In addition, the target population needs to be determined. For the asymptomatic, average-risk screening population, it is important to make a proper selection, i.e. reaching for high specificity. High sensitivity is also of importance for screening tests, and it needs to be kept in mind that the likelihood that a positive screening test will give the correct result (positive predictive value) is also depending on the prevalence of the disease in the population. ${ }^{29}$

When considering the increased-risk surveillance population (post-CRC, postpolypectomy or familial risk) sensitivity is of utmost importance, since especially false negative results are undesirable while higher rates of false positivity can be accepted. A lower specificity should be acceptable in order to increase test sensitivity, even more since in current surveillance program all patients undergo colonoscopy.

With the goal of possible implementation of mt-sDNA testing the surveillance population, comparison to FIT tests (currently: OC Sensor and FOB Gold) is required. This is being evaluated in the context of the MOCCAS study (MOlecular Stool Testing For Colorectal CAncer Surveillance). ${ }^{28}$

In the interim analysis (chapter 6) we have shown that the mt-sDNA test has a higher sensitivity than FIT for detection of advanced neoplasms (AN) in the surveillance population, which can be attributed to the DNA marker component of the test. It appeared that in the stool samples of patients with advanced adenomas (AA), especially those with advanced serrated polyps (ASP) as most advanced lesion, stool $\mathrm{Hb}$ concentrations were low.

Delayed detection may be acceptable for AA and ASP considering the slow rate of malignant transformation, but for CRC this is debatable. Repeated testing can increase the likelihood of detection within a given timeframe and can reduce the number of missed lesions. 
In line with this, other initiatives to optimize current surveillance programs are being explored, such as the optimal time interval for colonoscopy surveillance. Surveillance colonoscopies should be offered at a frequency required to provide adequate protection against development of cancer yet being cost-effective for providers and caregivers. ${ }^{32}$ The ongoing European Polyp Surveillance study (EPOS) study consists of two randomized controlled trails and an observational cohort study, where it is hypothesized that because of the improved colonoscopy quality, current surveillance intervals are too strict. Patients with different risks of CRC are randomized between shorter and longer surveillance intervals. ${ }^{33}$ After a follow-up of 10 years these intervals will be compared to reach the optimal surveillance interval between colonoscopies.

Another consequence of repeated testing is that more patients will have a positive test and subsequent colonoscopy. With the current cost difference between FIT and mt-sDNA test Cologuard ( $\sim 20$ vs. $\sim € 600$ ), it is not likely that the repeated testing using this test will become cost-effective. Especially since current colonoscopy costs $(\sim € 450)$ are lower than the Cologuard-costs, and subsequent colonoscopy is needed after a positive mt-sDNA test. In addition, practical considerations regarding the mtsDNA test, as drop-out rates, logistic and analytical problems need to be considered.

The surveillance group is very heterogeneous, therefore a more personalized approach is needed. The surveillance population in the MOCCAS study consists of patients with a history of CRC or polypectomy, and familial risk for CRC. The postCRC group has a higher risk of CRC at colonoscopy (1.7\%), compared to the postpolypectomy $(0.4 \%)$ and familial risk group (zero). It is worthwhile to develop tailored surveillance strategies for the various subgroups of the surveillance population. Another option could be by adapting the cut-off value of the test, based on individual clinical and/or pathological risk factors, such as age, gender, life-style factors or previous colonoscopy findings. 
After completion of the MOCCAS study, mathematical modelling will be performed to identify the optimal stool-based surveillance strategy. In addition, the long-term efficacy of stool-based surveillance on CRC mortality and impact on the colonoscopy burden needs to be assessed.

\section{Colonoscopy}

Colonoscopy is a crucial but invasive tool for (early) CRC diagnosis. It allows direct visualization, including detection and determination of colorectal (precursor) lesions and provides the opportunity to take biopsies and resect lesions.

In chapter 3 the use colonoscopy for polyp detection was addressed for patients with Lynch Syndrome (LS), the most prevalent hereditary form of CRC with a lifetime risk of developing CRC up to $80 \% .^{34}$ Therefore, intensive colonoscopy surveillance is recommended. ${ }^{35}$ Image enhancement using (virtual) chromoendoscopy (CE) is suggested to optimize the quality of surveillance, however there are only a few studies on CE in LS patients. Unfortunately, these studies are very heterogeneous regarding study design, timing of polypectomy and different types of image modalities. Therefore, a meta-analysis could not be performed and firm conclusions cannot be drawn.

In this respect new developments in the field of colonoscopy need to be taken into account. Nowadays, most endoscopy units use high-definition white light endoscopy (HD-WLE), which allow visualization in more detail. Additionally, the virtual chromoendoscopy techniques (i.e. narrow-band imaging (NBI), I-scan, autofluorescence endoscopy (AFE)) are being improved and can be easily applied in regular care. ${ }^{36}$

With regard to polyp detection and characterization, an important role is foreseen for artificial intelligence (AI). Computer-aided detection (CAD) algorithms are developed and have the potential to provide automated detection of colorectal polyps. Recent studies show promising results in increasing adenoma detection 
rates using AI mechanisms. ${ }^{37-39}$ Next to polyp detection, artificial intelligence might also play a role in the determination (i.e. estimating the histology) of polyps.

In current clinical practice, all lesions found during colonoscopy are removed and sent in for histological evaluation, adding substantially to healthcare costs. The majority of the polyps found during the bowel cancer screening program (BCSP) is however small $(\leq 10 \mathrm{~mm})$ or even diminutive $(\leq 5 \mathrm{~mm})$, harboring a very low risk of cancer. $^{40}$

Optical diagnosis (i.e. the in vivo estimation of polyp histology) is considered a costeffective strategy for diminutive polyps ${ }^{41}$ and can be safely implemented if criteria are met: $\geq 90 \%$ negative predictive value (NPV) for adenomatous histology and $\geq 90 \%$ agreement on surveillance intervals. ${ }^{42,43}$ In study settings in expert centers with highly trained endoscopists, meeting these criteria comes into sight. ${ }^{44}$ On the other hand, in regular, real-life BCSP practice (chapter 4) the NPV for optical diagnosis were clearly not met. It is therefore too early to start implementation of the 'diagnose and leave strategy' in clinical care. However, for the 'resect and discard strategy' the criteria are met.

Although quality criteria have been set for endoscopists performing colonoscopy within the setting of $\operatorname{BCSP}^{45}$ (Box 2-A), limitations need to be acknowledged and improvements can be made. Data for this study came directly from endoscopy reports in the BCSP format, where data on the 'level of confidence' of optical diagnosis are missing, as well as of the use of image-enhancement. In addition, no detailed classification system for optical diagnosis was used. Literature data show that the above mentioned thresholds can be met a) when only polyps optically diagnosed with high-confidence are considered and b) by expert endoscopists that have additionally been trained. ${ }^{44}$

So, if the above-mentioned limitations can be addressed, actual implementation of optical diagnosis in current clinical practice might become feasible (Box 2-B). 
Box 2. Overview for quality of colonoscopy and optical diagnosis

Box 2-A: Quality criteria for the BCSP in the Netherlands

- Qualifications and experience:

o Professional registration and accreditation of endoscopists

o Number of colonoscopies ( $\geq 500$ lifetime*, $\geq 200 /$ year**) $^{* *}$

o Number of polypectomies ( $\geq 50$ lifetime*, $\geq 50 /$ year*)

- Completeness of examination:

o Cecal intubation rate $(\geq 90 \% *, \geq 95 \% * *)$

o Bowel preparation (Boston Bowel Preparation Scale $\geq 6$ is considered adequate; $\geq 90 \%{ }^{* *}$ )

o Withdrawal time (at least 6 minutes; $\geq 90 \% * *$ )

- Detection and removal rates:

o Adenoma detection rate $(\geq 20 \% *, \geq 30 \% * *)$

o Polyp removal rate (polyps removed relative to polyps detected; $\geq 90 \% * * *$ )

*Accreditation criterion

** Audit criterion

This is a selection of the quality criteria by the Dutch Colonoscopy Quality Assurance working group ${ }^{47}$

Box 2-B: Recommendations regarding (implementation of) optical diagnosis

- Optical diagnosis:

o Additional endoscopist training in optical diagnosis

- Reporting:

o Use a detailed, standardized endoscopy reports, including:

- Validated classification systems to describe the lesion

- Level of confidence of optical diagnosis

- Use of image-enhancement, including photo-documentation

- Equipment:

o High-definition endoscopes and equipment (HD-cabels, HD-screens)

o Endoscopes with features allowing image-enhancement

- Implementation:

o Location-based strategies and other strategies to make optical diagnosis strategies more feasible need to be explored

To safely implement the optical diagnosis strategies, it is important to consider the consequences of this strategy.

Incorrect optical diagnosis for the 'diagnose and leave strategy' is unfavorable, since this means leaving potential premalignant lesions in situ in the rectosigmoid area. Although the transition from adenoma to carcinoma is of long duration ${ }^{4}$, it is also known that early cancers occur more often in the rectosigmoid area. ${ }^{46}$

For the 'resect and discard strategy' the consequences are less unfavorable. According to this strategy (adenomatous) polyps are always removed, but not sent in for histological evaluation. 
Moreover, recent literature shows that the risk of metachronous lesions does not increase with diminutive polyps harboring advanced histologic features. ${ }^{47}$

It needs to be kept in mind that the criterium for implementation ( $\geq 90 \%$ agreement on surveillance) is debatable, since surveillance intervals are mainly based on presence of larger polyps.

Current strategies distinguish between hyperplastic polyps and adenomas, but there is growing evidence that sessile serrated lesions (SSLs) are also precursor lesions, arising from the serrated neoplasia pathway. SSLs are more prevalent than previously assumed (7.3\% in screening population) ${ }^{48}$ and account for $15 \%$ of the CRCs. ${ }^{49}$

Not including SSPs in optical diagnosis regimens may have a substantial influence on the feasibility of the optical diagnosis strategies. Since it was shown in a recent study that optical diagnosis for SSLs was accurate in only $24.4 \%$, more training and research is needed to finally include the differentiation of SSLs in optical diagnosis regimens..$^{50}$

In the surveillance population studied in the MOCCAS study (chapter 6) advanced serrated polyps were present, where not less than 55 of 180 patients had an advanced serrated polyp as most advanced lesion. This emphasizes the importance of taking these lesions into consideration, both for (optical) diagnosis, as well as surveillance.

The Adenoma and Serrated Pathway to Colorectal CAncer (ASCCA) model, takes both the adenoma-carcinoma and the serrated pathway into account. ${ }^{51}$ When via this model the FIT-based screening vs. optical diagnosis for diminutive polyps were simulated, it revealed that costs could be saved without decreasing program effectiveness. ${ }^{41}$ To actually accommodate implementation, steps need to be taken to make the model more easily applicable and feasible.

For this matter, alternative criteria for implementation have been are suggested, since current PIVI criteria have drawbacks in clinical practice. ${ }^{52}$ 
The $\geq 90 \%$ NPV for adenomatous histology needs to be determined with highconfidence, which is not reported in the standard CRC screening endoscopy report. In addition, trends occur towards implementation by endoscopists of a 'diagnose and leave strategy' of hyperplastic polyps in the rectosigmoid. It is well known that surveillance intervals are predominantly depending on the presence of large, non-diminutive polyps. Preliminary results from Houwen et al. indicate that when endoscopists reach the 90\% NPV PIVI criteria, they also meet the surveillance agreement. ${ }^{52}$ When endoscopists are systematically trained in optical diagnosis ${ }^{44}$, implementation of above mentioned strategies should become feasible.

In addition, there is great potential for artificial intelligence and computeraided detection (CAD) systems in polyp determination, eliminating the interobserver variability of the endoscopist. Preliminary data on NPV for identification of diminutive adenomas show NPVs up to $97 \% .^{53,54}$

In general, artificial intelligence (AI) research within medicine is growing rapidly. Investments in medical AI projects are being made. Apart from excitement, there is also skepticism: AI algorithms do not provide us with causal relationships or underlying mechanistic insight and scientifically based evidence. ${ }^{55}$ Before implementation, these promising data have to be validated and confirmed in daily practice, causal relations need to be assessed, as well as classical scientific evidence should be provided.

Currently developed CAD systems are based on the best high-quality images, while this should include also poor-quality images, since the circumstances are not always perfect to obtain images of the best quality. Additionally, the CAD system needs to be widely available, i.e. for all different types of endoscopes from various manufacturers with the differences in imageenhancement options.

For the future research well-designed prospective studies using real-time use of $\mathrm{CAD}$, comparing CAD vs. no CAD should be performed. Taking into account different types of images, different types of endoscopes and different types of lesions 
(i.e. besides adenomas and hyperplastic polyps, also sessile serrated lesions or early and hereditary cancers). ${ }^{37}$ Where we stand now is that artificial intelligence might be a great support for doctors, but should not be seen as a replacement of medical expertise.

\section{Minimal invasive treatment}

With implementation of the Dutch BCSP more diminutive polyps are detected, but also more early (T1) CRCs are found during colonoscopy. ${ }^{46}$

For these lesions optical diagnosis is of great importance, since this is key for choosing the optimal treatment. Data from the Dutch BCSP (chapter 5) show poor optical recognition of T1CRCs in daily clinical practice (sensitivity of 20.9\%), which hinders optimal treatment decision and leads to surgical overtreatment.

Improvement in recognition of T1CRCs is needed. The OPTICAL study provided a validated risk chart, where based on several white-light and NBI-features, the CRC risk (in \%) can be estimated during colonoscopy. ${ }^{56}$ Implementation of the validated OPTICAL risk-chart provides guidance by estimating CRC-risk in daily colonoscopy practice.

Recognition of T1CRCs is of importance, since a subset of T1 CRCs, i.e. with lowrisk features, can be safely removed with endoscopic resection. However, in order to be curative, these lesions should be resected in an en-bloc fashion. ${ }^{57}$ This requires more advanced endoscopic resection techniques, such as endoscopic submucosal dissection (ESD). In contrast to endoscopic mucosal resection (EMR), en-bloc resection is possible with ESD even when polyps exceed the size of $20 \mathrm{~mm}$. Moreover, the advantage of ESD over EMR is the lower risk for recurrence (1-3\%). ${ }^{57,58}$ ESD is however associated with longer procedure time, a slightly increased risk of complications, especially a higher perforation risk, and it is more costly. ${ }^{57,59-61}$

Clinical studies on direct comparison of these techniques are currently ongoing. In the Netherlands, results are expected from the MATHILDA trial, a randomized controlled trial comparing EMR and ESD. ${ }^{62}$ 
Cost-effectiveness analysis (using decision tree modelling) comparing different treatment strategies show that it is cost-effective to perform ESD for lesions suspicious to contain submucosal invasive cancer, and pEMR for lesions $\geq 20 \mathrm{~mm}$ suspected to be non-invasive. ${ }^{63}$ Tools to estimate the CRC risk have not been taken into account in this analysis. Therefore, a model based on this analysis is not yet feasible for implementation in clinical practice. In general, it is known that the tools for endoscopic prediction of submucosal invasive cancer (SMIC) have limited accuracy, and that interobserver agreement varies across studies. ${ }^{64}$

For future cost-effectiveness studies, it would be interesting to determine at which percentage risk for T1CRC, more expensive and timely procedures such as ESD become cost-effective, using a validated estimation tool, for example the OPTICAL risk chart. ${ }^{56}$

In the process of optimizing quality and efficacy of CRC diagnosis and treatment, monitoring and quality control are important factors. Post-colonoscopy colorectal cancer (PCCRC) could be used as an indicator for quality; the PCCRC rate should be as low as possible. In chapter $\mathbf{7}$ endoscopist training is shown to be effective in reducing the post-colonoscopy colorectal cancer (PCCRC) rate. The occurrence of PCCRCs in this study could be related to missed lesions, non-adherence to surveillance intervals, inadequate bowel examination or newly developed cancers. Since the data collection of this study is until 2014, the PCCRC rate is likely to decrease even more, due to the introduction of more advanced endoscopy techniques ${ }^{65}$, as well as strict monitoring and training on behalf of the BCSP. ${ }^{66}$ 


\section{Concluding remarks}

Optimizing the quality and efficacy of CRC diagnosis and endoscopic therapy can only be achieved by joined efforts from researchers, various clinical specialists (both gastroenterologists and pathologists) and patients.

Much research is being performed, but translation to the clinic needs to be kept in mind in every step of the process. In this thesis we focused on several areas including less-invasive techniques for CRC diagnosis and minimal invasive treatment, as well as optimizing colonoscopy quality. We have shown that there is room for improvement in every area. Figure 1 shows the important areas of optimizing quality and efficacy of CRC diagnosis and endoscopic therapy.

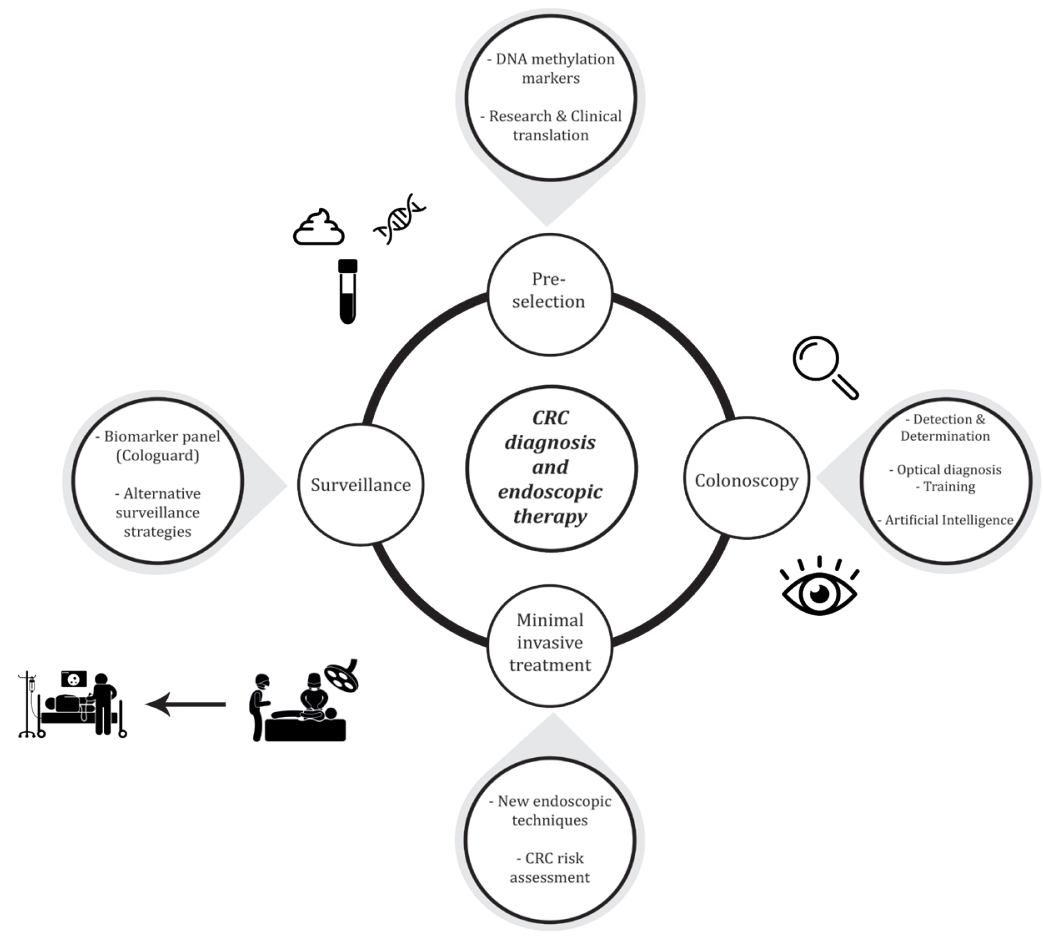

Figure 1. Outline of this thesis and future perspectives for CRC diagnosis and endoscopic therapy 


\section{References}

1. Siegel RL, et al. Cancer statistics, 2019. CA Cancer J Clin 2019;69(1):734.

2. Torre LA, et al. Global cancer statistics, 2012. CA Cancer J Clin 2015;65(2):87-108

3. Arnold M, et al. Global patterns and trends in colorectal cancer incidence and mortality. Gut 2017;66(4):68391

4. Kuntz KM, et al. A systematic comparison of microsimulation models of colorectal cancer: the role of assumptions about adenoma progression. Med Decis Making 2011;31(4):530-9.

5. Winawer SJ, et al. Prevention of colorectal cancer by colonoscopic polypectomy. The National Polyp Study Workgroup. N Engl J Med 1993;329(27):1977-81.

6. Denters MJ, et al. Patient burden of colonoscopy after positive fecal immunochemical testing for colorectal cancer screening. Endoscopy 2013;45(5):342-9.

7. Tinmouth J, et al. Faecal immunochemical tests versus guaiac faecal occult blood tests: what clinicians and colorectal cancer screening programme organisers need to know. Gut 2015;64(8):132737.

8. Navarro M, et al. Colorectal cancer population screening programs worldwide in 2016: An update. World J Gastroenterol 2017;23(20):3632-42
9. Schreuders EH, et al. Colorectal cancer screening: a global overview of existing programmes. Gut 2015;64(10):1637-49.

10. Oort FA, et al. Colonoscopycontrolled intra-individual comparisons to screen relevant neoplasia: faecal immunochemical test vs. guaiac-based faecal occult blood test. Aliment Pharmacol Ther 2010;31(3):432-9.

11. Gies A, et al. Direct Comparison of Diagnostic Performance of 9 Quantitative Fecal Immunochemical Tests for Colorectal Cancer Screening. Gastroenterology 2018;154(1):93-104.

12. Toes-Zoutendijk E, et al. Real-Time Monitoring of Results During First Year of Dutch Colorectal Cancer Screening Program and Optimization by Altering Fecal Immunochemical Test CutOff Levels. Gastroenterology 2017;152(4):767-75 e2.

13. Imperiale TF, et al. Performance Characteristics of Fecal Immunochemical Tests for Colorectal Cancer and Advanced Adenomatous Polyps: A Systematic Review and Meta-analysis. Ann Intern Med 2019.

14. You JS, Jones PA. Cancer genetics and epigenetics: two sides of the same coin? Cancer Cell 2012;22(1):9-20.

15. Menter DG, et al. Back to the Colorectal Cancer Consensus Molecular Subtype Future. Curr Gastroenterol Rep 2019;21(2):5. 
16. Guinney J, et al. The consensus molecular subtypes of colorectal cancer. Nat Med 2015;21(11):13506.

17. Jones PA, Baylin SB. The fundamental role of epigenetic events in cancer. Nat Rev Genet 2002;3(6):415-28.

18. Illingworth RS, Bird AP. CpG islands - 'A rough guide'. Febs Letters 2009;583(11):1713-20.

19. Taby R, Issa JPJ. Cancer Epigenetics. Ca-a Cancer Journal for Clinicians 2010;60(6):376-92.

20. Costa FF. Non-coding RNAs, epigenetics and complexity. Gene 2008;410(1):9-17.

21. Croce CM. Causes and consequences of microRNA dysregulation in cancer. Nature Reviews Genetics 2009;10(10):704-14.

22. Vacante $\mathrm{M}$, et al. Biomarkers in colorectal cancer: Current clinical utility and future perspectives. World J Clin Cases 2018;6(15):86981.

23. Chauvin A, Boisvert FM. Clinical Proteomics in Colorectal Cancer, a Promising Tool for Improving Personalised Medicine. Proteomes 2018;6(4).

24. Di Lena $\mathrm{M}$, et al. Volatile organic compounds as new biomarkers for colorectal cancer: a review. Colorectal Dis 2016;18(7):654-63.
25. Lieberman D. Colon-polyp surveillance--do patients benefit? N Engl J Med 2014;371(9):860-1

26. Greuter MJE, et al. Screening for Colorectal Cancer With Fecal Immunochemical Testing With and Without Postpolypectomy Surveillance Colonoscopy: A CostEffectiveness Analysis. Ann Intern Med 2017;167(8):544-54.

27. Imperiale TF, et al. Multitarget stool DNA testing for colorectalcancer screening. N Engl J Med 2014;371(2):187-8.

28. van Lanschot MC, et al. Molecular stool testing as an alternative for surveillance colonoscopy: a crosssectional cohort study. BMC Cancer 2017;17(1):116.

29. World Health Organization (WHO). Cancer - Screening for various cancers, via: https://www.who.int/ cancer/detection/variouscancer/. 2019.

30. Sanduleanu S, et al. Definition and taxonomy of interval colorectal cancers: a proposal for standardising nomenclature. Gut 2015;64(8):1257-67.

31. Stoffel EM, et al. Clinical and Molecular Characteristics of Post-Colonoscopy Colorectal Cancer: A Population-based Study. Gastroenterology 2016;151(5):8708 e3. 
32. European Colorectal Cancer Screening Guidelines Working Group, et al. European guidelines for quality assurance in colorectal cancer screening and diagnosis: overview and introduction to the full supplement publication. Endoscopy 2013;45(1):51-9.

33. Jover $\mathrm{R}$, et al. Rationale and design of the European Polyp Surveillance (EPoS) trials. Endoscopy 2016;48(6):571-8.

34. Vasen HF. Review article: The Lynch syndrome (hereditary nonpolyposis colorectal cancer). Aliment Pharmacol Ther 2007;26 Suppl 2:113-26.

35. Stoffel EM, et al. Prevalence and predictors of appropriate colorectal cancer surveillance in Lynch syndrome. Am J Gastroenterol 2010;105(8):1851-60.

36. McGill S, et al. Image-enhanced endoscopy in practice. Can J Gastroenterol 2009;23(11):741-6.

37. Kudo SE, et al. Artificial intelligence and colonoscopy: Current status and future perspectives. Dig Endosc 2019.

38. Misawa M, et al. Artificial Intelligence-Assisted Polyp Detection for Colonoscopy: Initial Experience. Gastroenterology 2018;154(8):2027-9 e3.
39. Wang P, et al. Real-time automatic detection system increases colonoscopic polyp and adenoma detection rates: a prospective randomised controlled study. Gut 2019.

40. Vleugels JLA, et al. Natural history of diminutive and small colorectal polyps: a systematic literature review. Gastrointest Endosc 2017;85(6):1169-76 e1.

41. Vleugels JLA, et al. Implementation of an optical diagnosis strategy saves costs and does not impair clinical outcomes of a fecal immunochemical test-based colorectal cancer screening program. Endosc Int Open 2017;5(12):E1197-E207.

42. Rex DK, et al. The American Society for Gastrointestinal Endoscopy PIVI (Preservation and Incorporation of Valuable Endoscopic Innovations) on real-time endoscopic assessment of the histology of diminutive colorectal polyps. Gastrointest Endosc 2011;73(3):419-22.

43. ASGE Technology Committee, et al. ASGE Technology Committee systematic review and meta-analysis assessing the ASGE PIVI thresholds for adopting real-time endoscopic assessment of the histology of diminutive colorectal polyps. Gastrointest Endosc 2015;81(3):502 e1-e16. 
44. Vleugels JLA, et al. Effects of Training and Feedback on Accuracy of Predicting Rectosigmoid Neoplastic Lesions and Selection of Surveillance Intervals by Endoscopists Performing Optical Diagnosis of Diminutive Polyps. Gastroenterology 2018.

45. Bronzwaer MES, et al. Quality assurance of colonoscopy within the Dutch national colorectal cancer screening program. Gastrointest Endosc 2019;89(1):1-13.

46. Toes-Zoutendijk E, et al. Stage distribution of screen-detected colorectal cancers in the Netherlands. Gut 2018;67(9):17456.

47. Vleugels JLA, et al. Diminutive Polyps With Advanced Histologic Features Do Not Increase Risk for Metachronous Advanced Colon Neoplasia. Gastroenterology 2019;156(3):623-34 e3.

48. Hazewinkel Y, et al. Prevalence of serrated polyps and association with synchronous advanced neoplasia in screening colonoscopy. Endoscopy 2014;46(3):219-24.

49. JE IJspeert, et al. Serrated neoplasiarole in colorectal carcinogenesis and clinical implications. Nat Rev Gastroenterol Hepatol 2015;12(7):401-9.

50. Vleugels JLA, et al. Optical Diagnosis of Sessile Serrated Polyps: Bottleneck for the Optical Diagnosis Paradigm? J Clin Gastroenterol 2017;51(5):426-32
51. Greuter MJ, et al. Modeling the Adenoma and Serrated pathway to Colorectal CAncer (ASCCA). Risk Anal 2014;34(5):889-910.

52. Houwen B, et al. Guidance for Setting Alternative Competence Criteria for Optical Diagnosis of Diminutive Colorectal Polyps, Which Are Easier to Implement in Daily Practice - a Simulation Study. Gastrointestinal Endoscopy 2019;89(6):Ab152-Ab3

53. Mori Y, et al. Real-Time Use of Artificial Intelligence in Identification of Diminutive Polyps During Colonoscopy: A Prospective Study. Ann Intern Med 2018;169(6):357-66.

54. Byrne MF, et al. Real-time differentiation of adenomatous and hyperplastic diminutive colorectal polyps during analysis of unaltered videos of standard colonoscopy using a deep learning model. Gut 2017.

55. Buch VH, et al. Artificial intelligence in medicine: current trends and future possibilities. Br J Gen Pract 2018;68(668):143-4.

56. Backes Y, et al. Multicentre prospective evaluation of real-time optical diagnosis of T1 colorectal cancer in large non-pedunculated colorectal polyps using narrow band imaging (the OPTICAL study). Gut 2019;68(2):271-9. 
57. Fujiya M, et al. Efficacy and adverse events of EMR and endoscopic submucosal dissection for the treatment of colon neoplasms: a meta-analysis of studies comparing EMR and endoscopic submucosal dissection. Gastrointest Endosc 2015;81(3):583-95.

58. Wang J, et al. Endoscopic submucosal dissection vs endoscopic mucosal resection for colorectal tumors: a metaanalysis. World J Gastroenterol 2014;20(25):8282-7.

59. Fuccio L, et al. Why attempt en bloc resection of non-pedunculated colorectal adenomas? A systematic review of the prevalence of superficial submucosal invasive cancer after endoscopic submucosal dissection. Gut 2018;67(8):1464-74.

60. Moss A, et al. Endoscopic mucosal resection outcomes and prediction of submucosal cancer from advanced colonic mucosal neoplasia. Gastroenterology 2011;140(7):1909-18.

61. Dahan M, et al. What is the cost of endoscopic submucosal dissection (ESD)? A medico-economic study. United European Gastroenterol J 2019;7(1):138-45.
62. Backes Y, et al. Endoscopic mucosal resection (EMR) versus endoscopic submucosal dissection (ESD) for resection of large distal nonpedunculated colorectal adenomas (MATILDA-trial): rationale and design of a multicenter randomized clinical trial. BMC Gastroenterol 2016;16(1):56

63. Bahin FF, et al. Wide-field endoscopic mucosal resection versus endoscopic submucosal dissection for laterally spreading colorectal lesions: a costeffectiveness analysis. Gut 2018;67(11):1965-73.

64. Hayashi N, et al. Endoscopic prediction of deep submucosal invasive carcinoma: validation of the narrow-band imaging international colorectal endoscopic (NICE) classification. Gastrointest Endosc 2013;78(4):625-32.

65. Iwatate $\mathrm{M}$, et al. Post-colonoscopy colorectal cancer rate in the era of high-definition colonoscopy. World J Gastroenterol 2017;23(42):7609-17.

66. Rivero-Sanchez L, et al. Colorectal cancer after negative colonoscopy in fecal immunochemical test-positive participants from a colorectal cancer screening program. Endosc Int Open 2018;6(9):E1140-E8. 
GENERAL DISCUSSION 



\section{ADDENDUM}

Summary

Samenvatting

Valorization

List of publications

Curriculum Vitae

Dankwoord 


\section{Summary}

Worldwide, colorectal cancer (CRC) belongs to the top three occurring cancers and adds substantially to the cancer-related morbidity and mortality. When detecting and treating the precursor lesions in an early phase, morbidity and mortality can be reduced.

This thesis focusses on optimizing quality and efficacy of CRC diagnosis and endoscopic therapy, addressing four topics in this process; pre-selection, colonoscopy, minimal invasive treatment and surveillance (Figure 1).

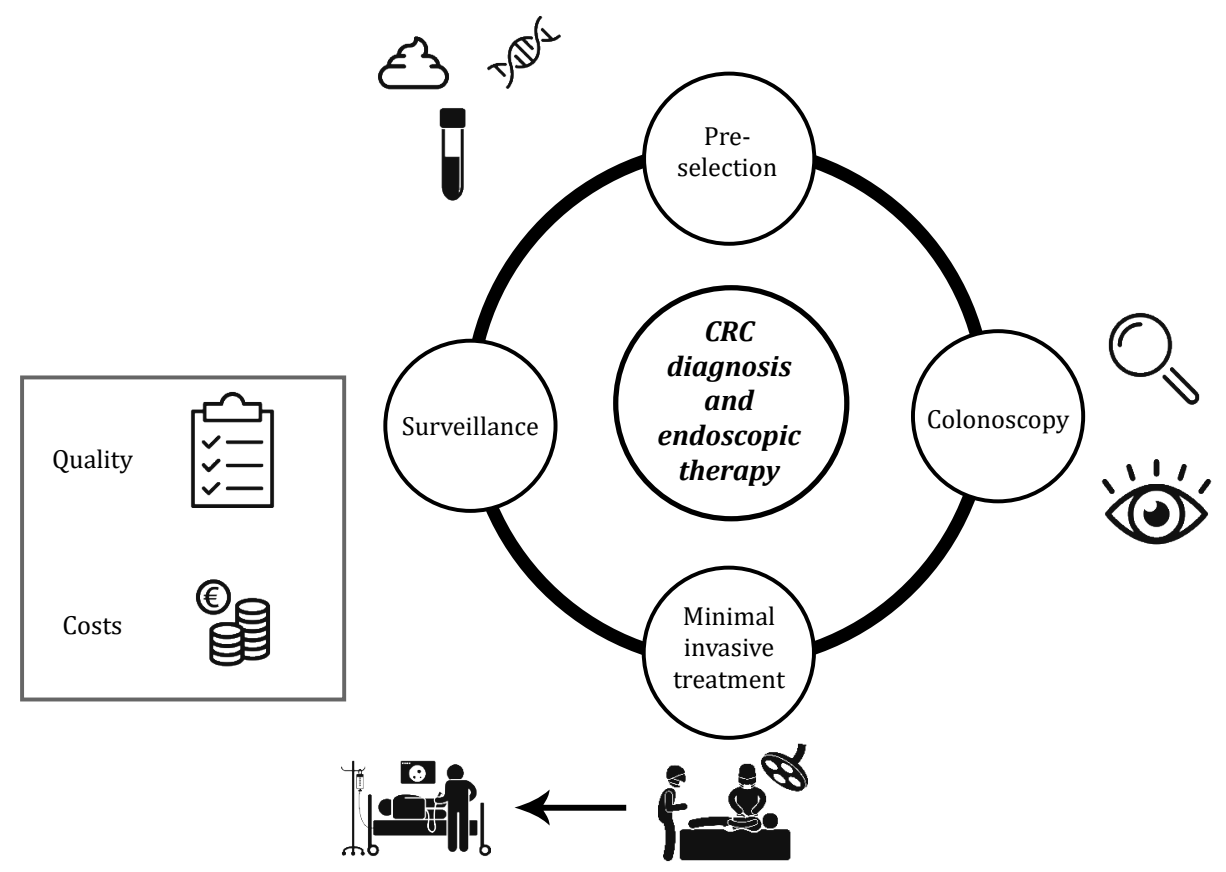

Figure 1. Overview of the topics of this thesis 
ADDENDUM

\section{Pre-selection}

In 2014, the nationwide colorectal cancer screening program was introduced in the Netherlands with the goal of reducing CRC-related mortality, by both detection of CRC in an early stage, as well as preventing CRC by detection and removal of precursor lesions.

Currently, fecal immunochemical testing (FIT) is widely implemented for CRC screening, detecting blood (hemoglobin) in the feces. After a positive FIT test, participants are invited for colonoscopy. This adds to the already pressured colonoscopy capacity, especially in the first years after implementation. FIT is reported to be $68-87 \%$ sensitive and $96-98 \%$ specific, but for precursor lesions the sensitivity is limited (23-40\%), while it is of utmost importance that these (precursor) lesions are detected. To improve or complement FIT tests, to optimize the pre-selection of patients who receive a colonoscopy and thereby decreasing the number and burden of colonoscopy capacity, biomarkers are widely investigated. The new insights on (molecular) biology of cancer had led to development of new diagnostic tests.

For this thesis we focused specifically on diagnostic DNA methylation markers. The review in chapter 2 showed that despite the numerous studies reporting on this topic, clinical translation is lacking. Only $0.8 \%$ of the studied diagnostic DNA methylation markers for CRC is used in current clinical practice.

The majority of the biomarkers (77\%) was studied in tissue (which can only be obtained via relatively invasive procedures), while for diagnostic purposes easily accessible specimen (e.g. stool or blood) is advised.

When looking at methodology of the biomarker studies, independent validation was scarce and only $42 \%$ of the bodily fluid markers was studied more than once. This points out that there is room for improvement and stresses the large gap between scientific research and implementation in clinical practice even more. 
To avoid the resulting 'research waste'; clinical needs, intended biomarker use and independent validation should be better considered prior to study design.

In addition, improved reporting quality would facilitate meta-analysis, thereby increasing level of evidence (LoE) and enabling clinical translation.

\section{Colonoscopy}

Colonoscopy plays an important role in the diagnosis and endoscopic treatment of precursors lesions and CRC, allowing direct visualization of the lesion and the opportunity to take biopsies or resect the lesion.

The additional value of image-enhancement, i.e. (virtual) chromoendoscopy (CE), is studied in a high-risk population of patients with Lynch Syndrome (LS). The (precursor) lesions in this patient group harbor different characteristics (i.e. non-polypoid and proximal location) and therefore the detection of these lesions is more difficult. In chapter 3 the additional value of CE in LS patients was assessed in a systematic review, but the large heterogeneity of the studies included in this review, i.e. large variety in study design, timing of polypectomy and different (virtual) CE techniques, does not allow a statistical analysis, which makes it hard to draw firm conclusions. For future research, a randomized cross-over back-to-back study design would be an efficient design for comparing two techniques, where each patient served as his/her own control. When using a second endoscopist, blinded for previous findings, the second look effect will be limited. In addition, training of endoscopists in detection of non-polypoid lesions was advised and the promising role of artificial intelligence, i.e. computer-aided detection algorithms needs to be determined.

In chapter 4 strategies for the determination of polyps were addressed. Optical diagnosis (i.e. the in vivo estimation of histology) of diminutive polyps $(\leq 5 \mathrm{~mm})$ may increase the efficacy and cost-effectiveness of colonoscopy. To adopt optical diagnosis strategies in clinical practice, the Preservation and Incorporation of Valuable endoscopic Innovations (PIVI) thresholds provide the basis to be met: 
$\geq 90 \%$ negative predictive value (NPV) for diagnosis of adenomatous histology and $\geq 90 \%$ agreement on surveillance intervals. We evaluated this within the Dutch Bowel Cancer Screening Program (BCSP).

For the 'resect and discard'-scenario, which applies to adenomatous polyps $\leq 5 \mathrm{~mm}$ in the entire colon (polyps are resected but not sent in for histologic evaluation) the criteria are met for the Dutch, European and American guideline.

For the 'diagnose and leave' strategy, hyperplastic polyps in the rectosigmoid are to be left in situ, the NPV was $84 \%$ (95\% CI 80-87). Therefore, the 'diagnose and leave' strategy does not meet the criteria. In conclusion, the accuracy of predicting histology remains challenging. Improvements in current BCSP practice need to be made regarding endoscopy equipment, additional training and monitoring, standardized classification systems, level of confidence of optical diagnosis and photo documentation.

Quality of colonoscopy is of great importance. Post-colonoscopy colorectal cancers (PCCRCs) are considered to be a crucial quality outcome measure for colonoscopy. PCCRCs may occur due to various reasons and non-polypoid colorectal neoplasms (NP-CRNs) and serrated lesions, smaller in size and more easily overlooked during colonoscopy, may be important precursor lesions of PCCRC.

Systematic training in detection and resection of NP-CRNs reduced the PCCRC rate by more than $50 \%$ (from 2.0/1000 colonoscopies to $0.8 / 1000$ colonoscopies) (chapter 7). From the 11 PCCRCs found after training, 45\% was associated with missed lesions, $18 \%$ was due to non-adherence to surveillance intervals, $18 \%$ due to newly developed CRC, $18 \%$ due to inadequate bowel examination and none due to incomplete polyp resection.

\section{Minimal invasive treatment}

The implementation of the Dutch BCSP has led to an increase in diagnosis of early (T1) CRC. However, endoscopic diagnosis of T1 CRC is challenging. In the study presented in chapter 5 we examined how well T1 CRC is recognised in the Dutch BCSP and the outcomes for therapy. 
Our data showed that only $20.9 \%(\mathrm{n}=24)$ of the T1 CRCs were endoscopically diagnosed as malignant. In the subanalysis is was shown that non-pedunculated T1CRCs were more easily recognized than pedunculated T1CRCs. To evaluate the the consequences, 91 initially non-recognized T1CRCs and their treatments were considered. It is concluded that incorrect optical diagnosis results in noncurative endoscopic resection, which might lead to unnecessary additional surgical treatment in low-risk cases.

Improvement in the recognition of T1CRCs is urgently needed. Implementation of the validated OPTICAL risk chart in daily practice provides guidance by estimating CRC risk. It should even be considered as a standardized component of the BCSP training and accreditation.

\section{Surveillance}

There is an increased risk of colorectal cancer (CRC) if CRC has been diagnosed previously, when a precursor lesion is removed in the past or if there is a familial risk of CRC. Therefore, these patients enter the surveillance program.

The current surveillance program for early detection of CRC in the Netherlands is colonoscopy-based. It is a major burden for the healthcare system while the costeffectiveness is questionable.

In the MOCCAS (MOlecular stool testing for Colorectal CAncer Surveillance) study, we aimed to assess whether the multitarget stool DNA test (Cologuard $®$ ) could be used to preselect patients with advanced neoplasia (AN) for surveillance colonoscopy. Cologuard performance was compared to the faecal immunochemical test (FIT) (OC Sensor and FOB Gold). In chapter $\mathbf{6}$ an interim analysis is presented.

The multitarget stool DNA test (mt-sDNA test) has higher sensitivity than FIT for detection of AN in the surveillance population. Applying a low test cut-off to reach $50 \%$ positivity rate (which is equal to decreasing the total colonoscopy capacity by half) the sensitivity of the mt-sDNA and FIT increased. In this scenario, the mtsDNA test missed only $24 \%$ of the advanced neoplasia, while FIT missed a higher proportion (40\%). Mathematical modelling approaches will be applied to the final 
study data in order to determine an optimal stool-based strategy for surveillance, in which costs are also taken into account.

\section{Conclusion}

In this thesis several topics are pointed out, with the goal of improving quality and efficacy of colorectal cancer diagnosis and endoscopic therapy. Four steps are identified this process; pre-selection, colonoscopy, minimal invasive treatment and surveillance. Although a lot of research in this field has been done, it is important that for every step translation towards clinical practice is considered. For each step mentioned above, there is room for improvement, where costs, quality and patient reported outcomes are essential parameters to take into account.

Optimizing quality and efficacy of colorectal cancer diagnosis and endoscopic treatment requires collaboration between different departments (gastroenterologists, radiologists, pathologists and surgeons) and patients.

Further research is needed to facilitate the implementation of the changes and improvements mentioned in this thesis in daily practice. 


\section{Samenvatting}

Wereldwijd, dikke darmkanker is de derde meest voorkomende kanker en draagt substantieel bij aan de ziektelast, gevolgen en beperkingen van kanker (morbiditeit) en de sterfte (mortaliteit) aan kanker. Indien voorloper afwijkingen (ook wel darmpoliepen genoemd) vroegtijdig worden gedetecteerd en behandeld, zal de morbiditeit en mortaliteit ten gevolge van dikke darmkanker significant dalen.

In dit proefschrift lag het accent op het optimaliseren van kwaliteit en effectiviteit van de diagnostiek van dikke darmkanker en van de endoscopische behandeling. Hierbij is dit proces onderverdeeld in vier stappen: pre-selectie, coloscopie, minimaal invasieve behandeling en surveillance (Figuur 1).

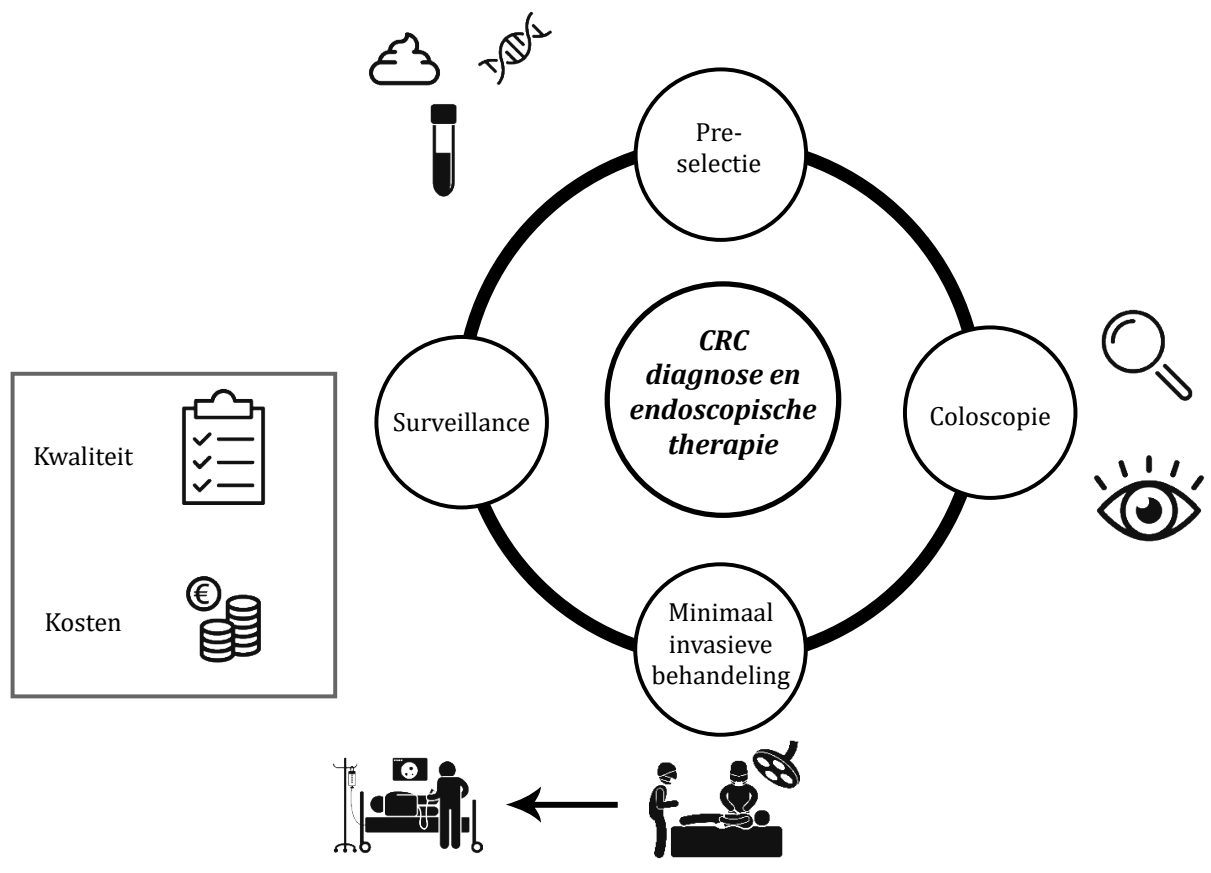

Figuur 1. Overzicht van de thema's in dit proefschrift 
ADDENDUM

\section{Pre-selectie}

In Nederland is in 2014 het landelijk bevolkingsonderzoek naar dikke darmkanker ingevoerd, met als doel de darmkanker sterfte terug te dringen door enerzijds darmkanker in een vroegtijdig stadium op te sporen, en anderzijds door darmkanker te voorkómen door darmpoliepen op te sporen en te verwijderen.

Momenteel wordt het bevolkingsonderzoek darmkanker uitgevoerd door middel van een fecale immunochemische test (FIT), welke bloed (hemoglobine) in de ontlasting opspoort. Na een positieve FIT-test worden deelnemers uitgenodigd voor een kijkonderzoek van de darm (coloscopie). Het bevolkingsonderzoek is stapsgewijs ingevoerd en drukte in de eerste jaren aanzienlijk op de reeds gelimiteerde coloscopie capaciteit. FIT is $68-87 \%$ sensitief (het percentage terecht positieve testuitslagen bij 'zieke' mensen) en 96-98\% specifiek (het percentage terecht negatieve testuitslagen bij mensen die 'niet ziek' zijn). Voor voorlopers van darmkanker (adenomen en serrated poliepen) ligt deze sensitiviteit een stuk lager (23-40\%), terwijl het juist belangrijk is om deze afwijkingen op te sporen.

Om de voorspellende waarden van de FIT test te verbeteren, en daarmee de preselectie van patiënten die worden uitgenodigd voor coloscopie te optimaliseren, wordt momenteel veel onderzoek gedaan naar biomarkers. Het toenemend inzicht in de biologische en moleculaire processen van kanker leidt tot het ontwikkelen van nieuwe diagnostische testen.

In dit proefschrift lag de focus specifiek op diagnostische DNA methylerings markers. Het review in hoofdstuk 2 laat zien dat ondanks het vele onderzoek op dit terrein, de vertaalslag van deze markers vanuit onderzoek-setting naar de klinische praktijk nog ontbreekt. Slechts $0.8 \%$ van de onderzochte diagnostische DNA methylerings markers voor darmkanker wordt op dit moment gebruikt in de klinische praktijk.

Het grootste deel van de biomarkers (77\%) is onderzocht in weefsel (wat alleen tijdens relatief invasieve procedures verkregen kan worden), terwijl voor een diagnostisch doel juist materiaal nodig is waar gemakkelijk toegang toe is, zoals 
ontlasting of bloed. Als we de kwaliteit van de studies die biomarkers onderzoeken in ogenschouw nemen, blijkt de onafhankelijke validatie schaars en slechts $42 \%$ van de in diverse lichaamsvloeistoffen onderzochte markers, is meer dan één keer onderzocht. Dit laat zien dat er nog ruimte is voor verbetering in kwaliteit van deze studies en dit toont tevens de kloof tussen wetenschappelijk onderzoek en de toepassing in de klinische praktijk.

Om deze 'research waste' te voorkomen, moet er over klinisch nut, beoogde doel van de biomarkers en onafhankelijke validatie beter worden nagedacht, bij voorkeur voorafgaand aan het opzetten en uitvoeren van studies. Daarnaast zou betere kwaliteit in de rapportage van studies helpen bij het uitvoeren van een meta-analyse (een statistische methode die verschillende studie resultaten combineert om duidelijker en statistisch sterker bewijs te verkrijgen). Dit gezamenlijk kan leiden tot een betere vertaling naar de klinische praktijk.

\section{Coloscopie}

Coloscopie speelt een belangrijke rol bij de diagnose en behandeling van (voorlopers van) dikke darmkanker. Coloscopie zorgt voor directe visualisatie van een afwijking (poliep) in de darm en geeft de mogelijkheid om biopten (stukjes weefsel) te nemen en om de poliep te verwijderen.

De toevoegde waarde van beeldoptimalisatie, door middel van (virtuele) chromoendoscopie (CE) - een kleuringstechniek gebruikt voor betere visualisatie van afwijkingen in de darm - werd onderzocht in een hoog-risico populatie van patiënten met Lynch Syndrome (LS). De (voorloper) afwijkingen in deze patiëntengroep bevatten andere karakteristieken (ze zijn niet-gesteeld en kennen een meer proximale locatie); wat de herkenning van dit type afwijkingen lastiger maakt. In hoofdstuk 3 werd de toegevoegde waarde van CE in patiënten met LS onderzocht middels een systematische review. Echter, door de grote verschillen (heterogeniteit) van de studies die zijn meegenomen in dit review, was het niet 
mogelijk om duidelijke conclusies te trekken. Voor toekomstig onderzoek, zou een "gerandomiseerde cross-over, back-to-back" studie opzet, de aangewezen opzet zijn om twee technieken te vergelijken, waarbij de patiënt twee keer een coloscopie ondergaat, waardoor hij/zij dient als zijn/haar eigen controle. Indien er een tweede endoscopist het onderzoek uitvoert, die geblindeerd is voor bevindingen tijdens de eerste coloscopie, zal het zogenaamde 'second look' effect (het idee dat als men twee keer kijkt, men überhaupt meer ziet) geminimaliseerd worden. Daarnaast wordt training van endoscopisten, specifiek gericht op de herkenning van niet-gesteelde laesies, geadviseerd. Ook is er in de toekomst een veelbelovende rol weggelegd voor 'kunstmatige intelligentie' (computer geassisteerde herkenningssystemen). De precieze rol hiervan zal in toekomstig onderzoek moeten worden bepaald.

In hoofdstuk 4 werden strategieën voor inschatting/bepaling van poliepen onderzocht. Optische diagnostiek (het met het oog inschatten van de histologie (=weefselonderzoek)) van kleine (diminutieve, $\leq 5 \mathrm{~mm}$ ) poliepen zou mogelijk de doeltreffendheid en kosteneffectiviteit van coloscopie kunnen verhogen. Om deze optisch diagnostiek strategieën uiteindelijk te implementeren in de klinische praktijk, zijn er criteria opgesteld. Deze criteria stellen dat er een $\geq 90 \%$ negatief voorspellende waarde (NPV - het aantal personen met een negatieve testuitslag zonder de desbetreffende ziekte) voor een bepaald type afwijking (adenomen) moet zijn, en $\geq 90 \%$ overeenstemming tussen het surveillance interval gebaseerd op optische diagnostiek in vergelijking met het surveillance interval gebaseerd op de histologie. Wij hebben dit onderzocht in het huidige Nederlandse darmkanker screening programma.

Voor de 'resect and discard' strategie, welke betrekking heeft op adenomen van $\leq 5 \mathrm{~mm}$ in de gehele darm (waarbij poliepen worden verwijderd en vervolgens niet voor histologische evaluatie ingestuurd worden), werd aan deze criteria voldaan, volgens zowel de Nederlandse, Europese als Amerikaanse richtlijn.

Voor de 'diagnose and leave' strategie, waar hyperplastische poliepen in het rectosigmoid niet worden weggehaald, was de NPV 84\% (95\% betrouwheidsinterval 
80-87\%). Voor deze strategie werden de hierboven genoemde criteria niet gehaald. Concluderend, de accuraatheid waarmee histologie voorspeld kan worden laat nog te wensen over. In het huidige bevolkingsonderzoek naar darmkanker is er nog ruimte voor verbetering, waaronder endoscopie materialen, extra training en monitoring, gestandaardiseerde classificatiesystemen, de mate van zekerheid van de optische diagnostiek en foto documentatie.

Ook is de kwaliteit van coloscopie van groot belang. Post-colonoscopie dikke darmkankers (PCCRCs - darmkanker ontstaan ondanks dat er een recente, negatieve coloscopie heeft plaatsgevonden) kunnen worden gezien als een cruciale uitkomstmaat voor het meten van kwaliteit van coloscopie. PCCRCs ontstaan door verscheidene oorzaken. Niet-gesteelde darmpoliepen (NP-CRNs) en geserreerde laesies, die kleiner zijn en dus sneller over het hoofd worden gezien bij coloscopie, zijn belangrijke voorlopers van PCCRCs.

Het systematische trainen in herkenning en behandeling van deze NP-CRNs blijkt het aantal PCCRCs met meer dan 50\% te verminderen (van 2.0/1000 coloscopieën naar 0.8/1000 coloscopieën) (hoofdstuk 7). Van de 11 PCCRCs die gevonden zijn ná de training was $45 \%$ geassocieerd met gemiste laesies, $18 \%$ vanwege het niet opvolgen van de surveillance-intervallen, $18 \%$ door nieuw ontstane dikke darmkanker, $18 \%$ door onvoldoende gereinigde darmen en geen van de PCCRCs ontstond door incomplete poliep verwijdering.

\section{Minimaal invasieve behandeling}

Met invoering van het Nederlandse bevolkingsonderzoek naar darmkanker worden er meer vroeg stadium kankers (T1 CRCs) gevonden, echter is de endoscopische diagnose van deze T1 CRCs lastig. In de studie in hoofdstuk 5 werd onderzocht hoe goed T1 CRCs worden herkend in het huidige bevolkingsonderzoek en wat de invloed hiervan is op de uitkomsten voor behandeling.

Onze data laten zien dat slechts $20.9 \%(n=24)$ van de T1CRCs endoscopisch werd gediagnostiseerd als kwaadaardig. In een subanalyse blijkt dat niet-gesteelde T1CRCs gemakkelijker te herkennen zijn dan gesteelde T1CRCs. 
Om de gevolgen hiervoor op de behandeling te evalueren, hebben we gekeken naar de 91 niet-herkende T1CRCs en de behandelingen die gedaan zijn. Hieruit kunnen we concluderen dat het niet herkennen van T1CRCs leidt tot niet-curatieve endoscopische resectie, hetgeen kan resulteren in onnodige chirurgie in patiënten met een laag-risico.

Verbetering in de herkenning van T1CRCs is nodig. De risico-tabel ontwikkeld in de OPTICAL-studie kan hierbij helpen, aangezien deze tabel handvaten biedt om het risico op kanker in te schatten. Daarom moet overwogen worden om deze OPTICALrisicotabel in de dagelijkse praktijk toe te passen, en wellicht zelfs onderdeel te maken van het standaard training- en toelatingsprogramma voor het bevolkingsonderzoek.

\section{Surveillance}

Als er in het verleden ooit darmkanker is geconstateerd, wanneer er een (risicovolle) poliep is verwijderd of in geval darmkanker in de familie voorkomt, is er een toegenomen kans dat deze patiënten (opnieuw) darmkanker ontwikkelen, en daarom worden zij opgevolgd via het surveillance programma. Het huidige surveillance programma in Nederland is gebaseerd op coloscopie, om zo darmkanker of voorlopers vroegtijdig op te sporen. Dit is een grote belasting voor het Nederlandse zorgsysteem, terwijl de kosteneffectiviteit hiervan niet bewezen is.

In de MOCCAS studie (MOlecular stool testing for Colorectal CAncer Surveillance), is het doel om te onderzoeken of de multitarget ontlasting DNA (mt-sDNA) test (Cologuard®) gebruikt kan worden om patiënten met gevorderde voorloperafwijkingen op te sporen en te pre-selecteren voor (surveillance) coloscopie. De opbrengst/prestatie van de mt-sDNA test werd vergeleken met verschillende ontlasting testen (FIT OC Sensor en FOB Gold). In hoofdstuk 6 is een tussentijdse (interim) analyse hierover gepresenteerd. De mt-sDNA test heeft een hogere sensitiviteit dan FIT voor het detecteren van gevorderde voorloperafwijkingen in de surveillance populatie. Indien er een lage test afkapwaarde wordt toegepast, om $50 \%$ positieven te behalen (hetgeen overeenkomt met een halvering van de totale coloscopie capaciteit), nam de sensitiviteit van de mt-sDNA test en van de FIT toe. 
In dit scenario miste de mt-sDNA test $24 \%$ van de gevorderde voorloperafwijkingen, terwijl FIT significant meer miste (40\%).

Zodra de studie afgerond is, zullen mathematische modelleringstechnieken worden toegepast, zodat de optimale ontlastingtest-gebaseerde strategie voor surveillance kan worden bepaald, waarbij ook de kosten en baten mee worden genomen.

\section{Conclusie}

In dit proefschrift zijn verschillende aspecten belicht en uitgediept, met als doel om de kwaliteit en effectiviteit van de diagnostiek van dikke darmkanker en van de endoscopische behandeling te verbeteren. Hierbij werden vier stappen geïdentificeerd: pre-selectie, coloscopie, minimaal invasieve behandeling en surveillance. Hoewel er veel onderzoek naar al deze stappen wordt gedaan, is het van belang dat bij iedere stap de vertaalslag naar de klinische praktijk wordt gemaakt. Voor iedere stap zoals hierboven beschreven is er ruimte voor verbetering, waarbij kosten, kwaliteit en ook de belasting voor de patiënt belangrijke pijlers zijn.

Daarnaast vergt het optimaliseren van de kwaliteit en effectiviteit van de diagnostiek van dikke darmkanker en van de endoscopische behandeling, een goede samenwerking tussen verschillende specialismen (waaronder MDL-artsen, radiologen, pathologen, chirurgen) en patiënten. Aanvullend onderzoek is nodig om de beoogde aanpassingen wat betreft diagnostiek en behandeling op adequate wijze te implementeren in de dagelijkse praktijk. 


\section{Valorization}

Colorectal cancer (CRC) is the third leading type of cancer worldwide, among both men and women. Since it is known that CRC derives from precursor lesions, detection and resection of these precursor lesions leads to a decrease in incidence, morbidity and mortality of CRC. With this goal, CRC screening programs have successfully been introduced and implemented. ${ }^{1}$ Patients with an increased risk to develop CRC (i.e. post-CRC status, post-polypectomy or familial/genetic risk) enter surveillance programs. $^{2}$

Valorization is described as "the process of creating value from knowledge by making knowledge suitable and available for societal and/or economic application by transforming it into products, services, processes and new business." ${ }^{3}$

In the framework of this thesis, we identified several steps in the process of CRC diagnosis and endoscopic therapy, following the patient journey in order to point out specific characteristics, strengths and weaknesses of each step (Table 1), so that recommendations can be made for future research and clinical practice.

By optimizing these steps, value is created through implementation of obtained knowledge into clinical practice, hence societal and economic application.

\begin{tabular}{|ll|}
\hline Pre-selection & $\begin{array}{l}\text { Which patients should be invited for a colonoscopy and what are the } \\
\text { best methods to identify patients (with precursor lesions) at risk for } \\
\text { CRC? }\end{array}$ \\
\hline Colonoscopy & $\begin{array}{l}\text { Invasive tool to detect precursor lesions with the option to resect these } \\
\text { lesions; How can we make sure that all (relevant) lesions are detected } \\
\text { and determined? How well is the endoscopist trained and equipped to } \\
\text { differentiate between the different types of lesions? }\end{array}$ \\
\hline Treatment & $\begin{array}{l}\text { Where surgery used to be the "gold standard" for treatment of CRC, } \\
\text { a shift occurs towards more advanced endoscopic treatments, that } \\
\text { potentially are less invasive and have lower complication rates } \\
\text { compared to surgery. How well can invasive lesions be differentiated } \\
\text { from non-invasive lesions? And what is the best, less-invasive } \\
\text { (endoscopic) technique to resect these (non) invasive lesions? }\end{array}$ \\
\hline Surveillance & $\begin{array}{l}\text { In current practice the surveillance population enters the colonoscopy- } \\
\text { based surveillance program. What are (less invasive) alternative } \\
\text { surveillance strategies or can intervals for colonoscopy be prolonged? }\end{array}$ \\
\hline
\end{tabular}

Table 1. Steps and questions in the process of optimizing quality and efficacy of CRC diagnosis and endoscopic therapy. 


\section{Value for patients}

For patients it is crucial that each of the four steps, described above, is effective and of high quality while the burden of each step should be as low as possible.

In current CRC screening programs, fecal immunochemical testing (FIT) is used for pre-selection. Since FIT based preselection is not optimal in detecting precursor lesions, it is essential to develop and test other diagnostic tools, where new biomarkers could possibly increase sensitivity and specificity and result in a more optimal preselection, so that a better selected group of patients is receiving a colonoscopy. Our systematic review (chapter 2) revealed that of the studies addressing diagnostic CRC DNA methylation markers, the majority of markers have been studied in tissue, which can only be obtained via colonoscopy. Bodily fluids (i.e. blood and stool) can be collected with a much lower burden to the patients, since these are less invasive to obtain. In case a minimal invasive test (with high sensitivity and specificity) is available, unnecessary (invasive) colonoscopies can be avoided.

To reach this goal, additional research is needed. For future DNA methylation biomarker studies it is important to define the clinical need and intended biomarker use before starting the study and to evaluate the current available research and the level of evidence. It is important that the specimen and cohort complement available evidence and that it is compared to current gold standard methods. This way the study adds substantially to the already performed research and can make a difference in bringing biomarkers to clinical practice.

Patients with a history of CRC or polypectomy, or familial/genetic risk enter into a surveillance program. Current surveillance is fully colonoscopy-based: patients receive colonoscopies on regular basis, thereby increasing the patient's burden. With implementation of nationwide CRC screening programs, and the increased quality of colonoscopy, the added value of intensive, high frequency colonoscopy-based surveillance programs is under debate. In this thesis we investigated an alternative surveillance method, using a multitarget stool DNA (mt-sDNA) test (Cologuard), including biomarkers and hemoglobin (MOCCAS study). The interim analysis (chapter 6) of that study shows that the mt-sDNA test has a higher sensitivity than 
FIT for precursor lesions in the surveillance population. When a low-test cut-off is used in order to reach $50 \%$ positivity rate, the mt-sDNA test sensitivity increases and the number of unnecessary colonoscopies will be reduced by $50 \%$. This interim analysis already shows promising results. After completion of the study, full analyses will be performed, and mathematical modelling will be applied to determine the optimal stool-based surveillance strategy.

Colonoscopy is an invasive procedure, which can be used for both diagnosis and treatment. Data from patient reported outcome measures (PROM's) and patient questionnaires show that bowel preparation and post-colonoscopy abdominal complaints are perceived as most burdensome by patients undergoing colonoscopy. ${ }^{4}$ Colonoscopy remains the most important tool for diagnosis of precursor lesions and for diagnosis of CRC. Colonoscopy allows us to take biopsies or resect (precursor) lesions. Optimization of colonoscopy procedures may help to significantly reduce patient burden while not affecting quality and efficacy.

To optimize the diagnostic process, adequate detection and determination of polyps is crucial. We investigated the additional value of image enhancement strategies in patients with Lynch Syndrome. These patients have a significantly increased risk of developing CRC. In these patients it is crucial to optimize the detection of the precursor lesions, so that development and progression to CRC can be prevented. In chapter 3 an overview of current literature was given, where a large heterogeneity among study designs was shown. For future research, standardization of study designs is needed, with a randomized, cross-over, back-to-back study design as best option. Important factors to increase the quality and efficacy of colonoscopy include 1) use of image-enhancement, 2) training of endoscopists in detection and removal of non-polypoid colorectal neoplasms and 3) stricter adherence to surveillance intervals.

The determination and in-vivo estimation of histology (i.e. optical diagnosis), was studied in the screening population. Two potential strategies in low-risk, diminutive $(\leq 5 \mathrm{~mm})$ polyps were investigated, aiming for less resections and saving costs by not 
sending these polyps for histological evaluation. According to our study (chapter 4), the accuracy for optical diagnosis of adenomatous histology is currently too low and does not consistently meet the set thresholds (PIVI criteria). Therefore, we are not ready to implement these strategies in current daily practice. For implementation of optical diagnosis in the CRC screening program, several adjustments need to be done: in the reporting of lesions by standard use of optical classification systems and by inclusion of the 'level of confidence' of the endoscopist performing the colonoscopy. Additional endoscopist training in optical diagnosis should be undertaken. Only when detection and determination have been optimized, colonoscopy as a diagnostic tool will reach optimum diagnostic accuracy and efficacy.

Nowadays, new endoscopic resection techniques are available. With implementation of the CRC screening program, more lesions are detected, and more early (T1) CRCs are diagnosed. For a subset of T1 CRCs, these less-invasive endoscopic treatments are alternative strategies for colorectal surgery. It is of great importance to correctly estimate the CRC risk in patients when choosing for the less-invasive treatment strategy. In current clinical practice however, only $20.9 \%$ of the T1 CRCs were recognized as such. Incorrect optical diagnosis results in non-curative endoscopic resections, this often leads to salvage surgery, which in a substantial number of cases appears to be unnecessary (chapter 5).

Implementation of the validated OPTICAL risk chart provides guidance by estimating CRC risk and should therefore be considered for implementation in daily practice. ${ }^{5}$

\section{Value for society}

In 2003, the European Committee advised all countries to implement CRC screening programs. After years of study, in 2014 the biannual FIT-based program was implemented in the Netherlands. ${ }^{1}$ In the first years of the program, colonoscopy capacity was limited due to a shortage of certified endoscopists and higher participation rates than expected. 
Because of the initially limited colonoscopy capacity and high participation rates among the population, waiting lists increased for CRC screening and also for symptomatic patients. Based on capacity issues, it was decided to increase the FIT cut-off value six months after implementation of the screening program. In 2018, four years after implementation, nationwide colonoscopy capacity is sufficient to accommodate all FIT positive referrals.

With implementation of the CRC screening program, more low-risk (small and diminutive) polyps are diagnosed and resected. In this thesis, the implementation of cost-effective strategies, i.e. 'resect and discard' and 'diagnose and leave' for diminutive polyps was studied. In case optical diagnosis can be optimized, diminutive polyps might be left in place or are resected but not sent in for histology. This strategy will result in substantial financial benefit.

As a consequence of implementation of the CRC screening program, more patients will enter colonoscopy-based surveillance programs. This results in a rapidly expanding surveillance population, adding substantially to colonoscopy capacity. Nowadays more than $25 \%$ of colonoscopies is performed for surveillance, while the cost-benefit of colonoscopy surveillance is not yet clear. Therefore, it is worthwhile to investigate alternative surveillance strategies. Better pre-selection of patients will lead to less (unnecessary) colonoscopies, with financial impact. Furthermore, the pressure on colonoscopy capacity will decrease and attention can be given to other important health issues in endoscopy practice such as innovation in therapeutic endoscopic interventions.

In the MOCCAS study (MOlecular stool testing for Colorectal CAncer Surveillance), an observational cross-sectional study, patients are approached to collect stool samples for molecular testing and FIT prior to their scheduled surveillance colonoscopy (chapter 6). The currently ongoing study will after completion, be used as input for mathematical modelling to find the optimal (stool-based) surveillance strategy. This should add to reduction of patient burden and increasing (cost-) effectiveness. 


\section{Overall and future value}

Diagnosing CRC in an early or precursor stage, may lead eventually to prevention of CRC. It is worthwhile to take steps to optimize this process, because by early diagnosis and treatment significant costs are saved from chemotherapy and surgery.

Steps taken to optimize the process of CRC diagnosis and treatment are of value, both for patients as well as society. The future value for each step is shown in Table 2 .

\begin{tabular}{|ll|}
\hline Pre-selection & $\begin{array}{l}\text { Role of diagnostic biomarkers/biomarker panels in the pre- } \\
\text { selection, complementary to the currently used FIT-test. }\end{array}$ \\
\hline Colonoscopy & $\begin{array}{l}\text { Optimal detection and determination of (pre) malignant lesions is } \\
\text { key. To improve this, endoscopist training is important, as well as } \\
\text { the potential role of artificial intelligence (AI) for correct optical } \\
\text { diagnosis. Further research and evidence is needed before AI can be } \\
\text { used in daily endoscopy practice. }\end{array}$ \\
\hline Treatment & $\begin{array}{l}\text { Shift from surgical to endoscopic/minimal invasive treatment } \\
\text { with a personalized plan for every patient/lesion, starting with } \\
\text { improvement of correct estimation and recognition of early CRCs. }\end{array}$ \\
\hline Surveillance & $\begin{array}{l}\text { Biomarkers may play a role in future surveillance strategies. } \\
\text { When determining the optimal personalized surveillance strategy, } \\
\text { several factors, e.g. risk differences in a heterogeneous population, } \\
\text { should be taken into account. }\end{array}$ \\
\hline
\end{tabular}

Table 2. Steps and potential ways for optimizing quality and efficacy of CRC diagnosis and endoscopic therapy.

In this thesis several aspects have been explored that may help to optimize quality and efficacy of CRC diagnosis and endoscopic therapy. Additional research is needed to adequately implement these adjustments in CRC diagnosis and treatment in daily practice. In this respect, costs and quality remain key factors. 


\section{References}

1. Rijksinstituut voor

Volksgezondheid en Milieu (RIVM).

Bevolkingsonderzoek Darmkanker.

Available via: http://www.rivm.nl/.

2. NVMDL. Dutch Guideline

Colonoscopy Surveillance 2013.

3. Maastricht.University. Valorisation

- Knowledge Transfer Office, via: https://www.maastrichtuniversity. nl/about-um/other-offices/ knowledge-transfer-office. 2019.

4. Denters MJ, et al. Patient burden of colonoscopy after positive fecal immunochemical testing for colorectal cancer screening. Endoscopy 2013;45(5):342-9..

5. Backes Y, et al. Multicentre prospective evaluation of real-time optical diagnosis of T1 colorectal cancer in large non-pedunculated colorectal polyps using narrow band imaging (the OPTICAL study). Gut 2019;68(2):271-9.

6. Kudo SE, et al. Artificial intelligence and colonoscopy: Current status and future perspectives. Dig Endosc 2019. 


\section{Publications}

L.W.T. Meulen, A.J.P. van de Wetering, M.P.H. Debeuf, Z. Mujagic, A.A.M. Masclee Optical diagnosis of T1 CRCs and treatment consequences in the Dutch CRC screening programme. Gut 2020 Published Online First: 14 January 2020. doi: 10.1136/ gutjnl-2019-320403

A.J.P. van de Wetering, L.W.T. Meulen*, R.M.M. Bogie*, Q.E.W. van der Zander, A. Reumkens, B. Winkens, H.R. Cheng, J.W.A. Straathof, E. Dekker, E. Keulen, C.M. Bakker, Ch.V. Hoge, R. de Ridder, A.A.M. Masclee, S. Sanduleanu. Optical diagnosis of diminutive polyps in the Dutch Bowel Cancer Screening Program; are we ready to start? Endoscopy International Open 2020; 08: E257-E265

A.J.P. van de Wetering, R.M.M. Bogie, A. Cabbolet, B. Winkens, A.A.M. Masclee, S. Sanduleanu. Update on the role of chromoendoscopy in colonoscopic surveillance of patients with Lynch syndrome. European Journal Gastroenterol Hepatology 2018;30(10):1116-24

A.J.P. van de Wetering, R.M.M. Bogie, C.M.C. le Clercq, A.A.M. Masclee, S. Sanduleanu. Impact of endoscopist training on postcolonoscopy colorectal cancer rate. Gastrointestinal endoscopy. 2017 May;85(5):1113-4.

Carine Vossen, Helen Vossen, Wiesje van de Wetering, Marco Marcus, Jim van Os and Richel Lousberg (May 9th 2012). The Use of Event-Related Potentials in Chronic Back Pain Patients, Low Back Pain, Ali Asghar Norasteh, IntechOpen,

DOI: 10.5772/35563. Available from: https://www.intechopen.com/books/lowback-pain/the-use-of-event-related-potentials-in-chronic-back-pain-patients 
ADDENDUM

To be submitted after completion of the MOCCAS study

M.C.J. van Lanschot, S.P. Rauh, M.J.E. Greuter, A.J.P. van de Wetering, B. Carvalho, P. Bossuyt, E. Dekker, G.A. Meijer, V.M.H. Coupe, on behalf of the MOCCAS study group*. Improving preselection for surveillance colonoscopy by complementing FIT with clinicopathological risk factors.

M.C.J. van Lanschot, A.J.P. van de Wetering, G.M. Lemmens, R.W.M. van der Hulst, A.M. van Berkel, A.C.T.M. Depla, M.E. van Leerdam, J.M. Jansen, C.A. Wientjes, J.W.A. Straathof, E.T.P. Keulen, D. Ramsoekh, B.M. Berger, G.P. Lidgard, M. Zacherl, S. Sanduleanu-Dascalescu, V.M.H. Coupé, A.A.M. Masclee, M. van Engeland, B. Carvalho, E. Dekker, G.A. Meijer. Multitarget stool DNA testing an faecal immunochemical testing (FIT) as alternative for colonoscopy-based surveillance: an interim analysis. 
PUBLICATIONS

\section{Scientific presentations}

A.J.P. van de Wetering, R.M.M. Bogie, A. Reumkens, L.W.T. Meulen, B. Winkens, H.R. Cheng, J.W.A. Straathof, E. Keulen, C.M. Bakker, C.V. Hoge, R. de Ridder, A.A.M. Masclee, S. Sanduleanu.

Optical diagnosis of diminutive polyps in the Dutch BCSP; are we ready to start?

United European Gastroenterology Week, Vienna, Austria, 2018 (oral presentation)

Antje van der Zee-Neuen, Annelies Boonen, Wiesje van de Wetering, Rob De Bie, Pieter Rodenburg, Nico Wolter, Sjef van der Linden.

Effects and costs of a return to work program for patients on recent sick-leave due to musculoskeletal disorders.

EU Medicine Assurance and Social Security, Maastricht, the Netherlands, 2018

(oral presentation)

A.J.P. van de Wetering, R.M.M. Bogie, A. Reumkens, L.W.T. Meulen, B. Winkens,

H.R. Cheng, J.W.A. Straathof, E. Keulen, C.M. Bakker, C.V. Hoge, R. de Ridder, A.A.M. Masclee, S. Sanduleanu.

Optical diagnosis of diminutive polyps in the Dutch BCSP; are we ready to start?

Digestive Disease Days, Veldhoven, the Netherlands, 2018 (oral presentation)

A.J.P. van de Wetering, A.C.O.G. Cabbolet, R.M.M. Bogie, B. Winkens, A.A.M. Masclee, S. Sanduleanu. Integration of Chromoendoscopy in Colonoscopic Surveillance of Patients with Lynch Syndrome: A Systematic Review and Meta-Analysis

Digestive Disease Week, Chicago, IL, United States, 2017 (poster)

A.J.P. van de Wetering, R.M.M. Bogie, C.M.C. Le Clercq, A.A.M. Masclee, S. Sanduleanu Impact of endoscopist training on postcolonoscopy cancer rate GROW Science Day, Maastricht, the Netherlands, 2017 (poster)

A.J.P. van de Wetering, A.C.O.G. Cabbolet, R.M.M. Bogie, B. Winkens, A.A.M. Masclee, S. Sanduleanu. Integration of Chromoendoscopy in Colonoscopic Surveillance of Patients with Lynch Syndrome: A Systematic Review and Meta-Analysis GROW Science Day, Maastricht, the Netherlands, 2016 (poster) 


\section{Curriculum Vitae}

Wiesje (Alouisa Johanna Petronella) van de Wetering was born on May 24st 1991 in Oss, the Netherlands. After graduating high school (Maasland College, Oss), she studied Psychobiology at the University of Amsterdam. In 2010, she was admitted to Medical School in Maastricht (Maastricht University). During her bachelor, she studied at Universita degli Studi di Ferrara, Italy. In her master, she did an internship gynecology and obstetrics in Mulago

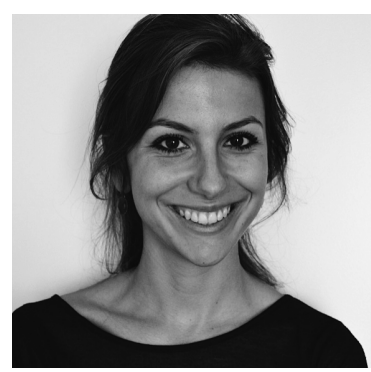
Hospital, Kampala, Uganda.

After acquiring her Medical Doctor's degree in 2016, she started her PhD project under supervision of dr. S. Sanduleanu, prof. dr. A.A.M. Masclee and prof. dr. M. van Engeland. As a PhD-fellow she was affiliated to the department of Gastroenterology and Hepatology, Maastricht University Medical Centre and GROW, School for Oncology and Developmental Biology, Maastricht University.

For three years, she worked on several studies concerning quality and efficacy of colorectal cancer diagnosis and endoscopic therapy and she coordinated the MOCCAS (MOlecular stool testing for Colorectal CAncer Surveillance) study for the Southern part of the Netherlands.

During her PhD project, she took place in several PhD courses and organized the Pélerin symposium twice, providing young doctors from the Maastricht UMC+ the opportunity to present their research. She was also closely involved in setting up an outpatient clinic for patients with chronic diseases, with focus on non-medical aspects and integral care. Here, her interest in Rheumatology was born.

As of 2020, she started her residency Internal Medicine in the Amsterdam UMC location AMC (under supervisor of prof. dr. S.E. Geerlings and prof. dr. J.M. Prins), as the first part of her residency program in Rheumatology (under supervisor of prof. dr. N. de Vries and dr. M. van Onna). 


\section{Dankwoord}

Het woord van dank. Aan iedereen die heeft bijgedragen aan de totstandkoming van dit proefschrift en aan iedereen die me dierbaar is.

Aan degenen die het vertrouwen in me hadden om dit traject te starten en me gedurende deze jaren hebben begeleid. Degenen die de tijd namen om te brainstormen, om kritisch naar mijn manuscripten te kijken en me inspireerden, ieder op zijn/haar eigen manier.

Aan degenen die zorgden dat de MOCCAS-studie naar behoren kon worden uitgevoerd, waarmee de samenwerking zeer prettig verliep waardoor de kleine problemen snel en adequaat konden worden opgelost.

Waarbij er naast productieve meetings gelukkig ook tijd was voor gezelligheid, onder het genot van Limburgse vlaai, een Bossche Bol of een speciaal biertje in de Amsterdamse zon.

Aan al degenen die mijn pad hebben gekruist tijdens dit promotietraject, die meewerkten, meedachten, input en feedback gaven en interesse toonde. Die de tijd namen en me waardevolle adviezen hebben gegeven.

Aan degenen die deel uitmaken van de beoordelingscommissie, die de tijd hebben vrijgemaakt om met een kritische blik dit proefschrift te beoordelen.

Aan degenen die zorgden voor een hoop gezelligheid op de UNS. Waar alle hoogtepunten maar ook frustraties konden worden besproken. Waarbij er gelukkig genoeg tijd was voor $(10.00 \mathrm{u})$ koffie met 'get lekkers' en tijd werd gemaakt om naast het werk al het bourgondische van Maastricht te ontdekken.

Waarmee op de fiets werd gestapt om het hoofd leeg te maken, om de nodige hoogtemeters te trotseren en een dappere poging werd gedaan om mij wegwijs te maken in het mooie Limburgse land. 
Waarmee congressen werden bezocht, waarbij er naast zenuwen en support, gelukkig ook genoeg tijd was voor ontspanning, sightseeing en gezelligheid. Wat een fantastische momenten hebben wij beleefd.

In het bijzonder aan degenen van kamer 5.567 en degenen die met enige regelmaat plaatsnamen op de praatstoel. Waarmee werd gelachen, geklaagd en waarmee toch best veel werd meemaakt. Wat ben ik blij dat jullie er voor me waren.

En aan degene die het meeste tijd met mij op de kamer heeft doorgebracht. Waarmee er naast gesprekken over poliepen ook genoeg tijd was voor veel gezelligen gekkigheid. Ik ben blij dat jij op deze dag naast me staat.

Jullie hebben mijn promotietijd echt verrijkt!

Aan degenen die in deze onstuimige tijden hebben bijgedragen aan een fijne en warme overgang van onderzoek naar kliniek en van Maastricht naar Amsterdam.

Aan mijn lieve vriendinnen, wat prijs ik mezelf gelukkig met jullie.

Aan degenen waarmee het ooit in het Brabantse begon, maar die ik gelukkig nog regelmatig zie. Die, ondanks de verschillende paden die we op zijn gegaan, geïnteresseerd blijven en er altijd voor me zijn.

Aan degenen waarmee tijdens de (studenten)tijd in Maastricht de basis is gelegd voor een bijzondere vriendschap. Waarmee met enige regelmaat keuzes worden geëvalueerd, prachtige reizen worden gemaakt, en vooral met volle teugen wordt genoten. Wat ben ik blij met jullie!

Aan die ene vriendin, die tijdens mijn jaren in Maastricht onmisbaar voor me was en waarmee elke dag een feestje is, als we maar zelf de slingers ophangen. Samen komen wij er wel. Ik ben blij dat jij op deze dag naast me staat. 
Aan degene die dit prachtige ontwerp heeft gemaakt, die de tijd en moeite heeft willen nemen om zich te verdiepen in de wereld van de poliepen.

Aan degenen die behoren tot de hechte familie waarin ik heb mogen toetreden. Waar ik me ontzettend welkom voel, waar iedereen wordt gewaardeerd en waar mooie gesprekken worden gevoerd onder het genot van heerlijk eten en een goed glas wijn. Of we nou in Breda, Engeland, Amsterdam of Kenia zijn, het is altijd een feestje met jullie. Ik kan me geen warmere (schoon)familie wensen.

Aan mijn liefste zusje, die me weet op te vrolijken, zaken weet te relativeren en bij wie ik altijd terecht kan. Ik ben ontzettend trots op de vrouw die jij geworden bent en op de manier waarop je door het leven danst.

Aan mijn ouders, door wie ik me onbezorgd op mijn eigen manier heb kunnen ontwikkelen en prachtige dingen heb kunnen ontdekken.

Aan mijn lieve vader, die me vol trots en interesse volgt, waarvoor niets te veel is en die me als geen ander met beide benen op de grond weet te houden.

Aan mijn lieve moeder, die altijd luistert en precies de goede dingen zegt, waarvan ik hoop dat ik haar fantastische voorbeeld ooit kan evenaren.

Aan diegene bij wie ik thuiskom en bij wie ik rust vind.

Liefste Bart, je inspireert me met de manier waarop je in het leven staat en naar de wereld kijkt, met je ambitie, je doorzettingsvermogen en met al je liefde.

Jouw steun, geduld en vertrouwen slepen mij door de dalen en samen met jou geniet ik intens van alle grote, maar vooral ook alle kleine dingen van het leven, en laten we dat vooral ook blijven doen. Je maakt mij zo onwijs gelukkig!

Mijn dank aan jullie allen is bijzonder groot.

Veel liefs, Wiesje 

